\title{
MICROFLUIDIC SINGLE SPERM ANALYSIS
}

\author{
Bjorn de Wagenaar
}

$19^{\text {th }}$ of February, 2016 
The research described in this thesis was carried out at the BIOS Lab on a Chip group of the MESA+ Institute for Nanotechnology and the MIRA Institute for Biomedical Technology and Technical Medicine at the University of Twente, Enschede, The Netherlands. The research was financially supported by the NWO Spinoza Prize of prof. dr. ir. A. van den Berg.

Committee members:

Chairman:

prof. dr. P.M.G. Apers

University of Twente

Promotor:

prof. dr. ir. A. van den Berg University of Twente

Assistant-promotors:

dr. ir. L.I. Segerink

University of Twente

dr. ir. W. Olthuis

University of Twente

Members:

prof. dr. J.G.E. Gardeniers

University of Twente

prof. dr. L.W.M.M. Terstappen

University of Twente

dr. B.M. Gadella

University of Utrecht

prof. dr. H. Morgan

University of Southampton

Title: $\quad$ Microfluidic single sperm analysis

Author: Bjorn de Wagenaar

ISBN: $\quad$ 978-90-365-4034-6

DOI: $\quad 10.3990 / 1.9789036540346$

URL: $\quad$ http://dx.doi.org/10.3990/1.9789036540346

Publisher: Gildeprint, Enschede, The Netherlands

Copyright (C) 2016 by Bjorn de Wagenaar, Enschede, The Netherlands. All rights reserved. 


\title{
MICROFLUIDIC SINGLE SPERM ANALYSIS
}

\section{DISSERTATION}

\author{
to obtain
}

the degree of doctor at the University of Twente,

on the authority of the rector magnificus,

prof. dr. H. Brinksma,

on account of the decision of the graduation committee,

to be publicly defended

on Friday the $19^{\text {th }}$ of February 2016 at $14: 45$

by

Bjorn de Wagenaar

born on 29 April 1987

in Leimuiden, The Netherlands 
This dissertation has been approved by:

Promotor: $\quad$ prof. dr. ir. A. van den Berg

Assistant-promotors: dr. ir. L.I. Segerink

dr. ir. W. Olthuis 
"Quaerite et invenietis"

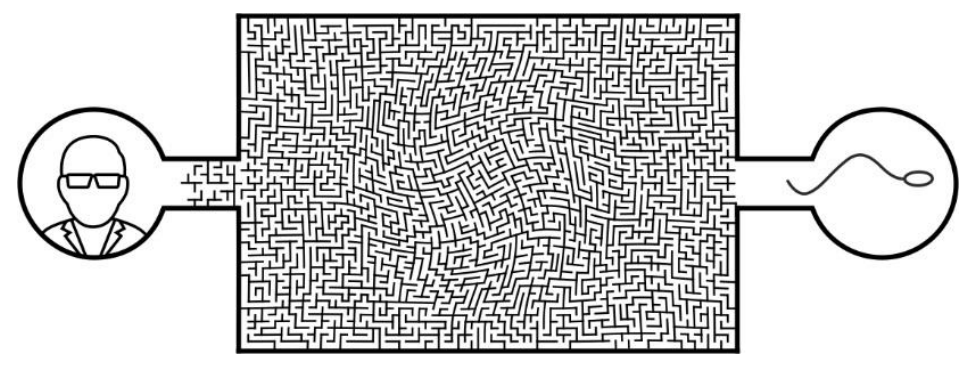





\section{Table of contents}

1 Aim and outline

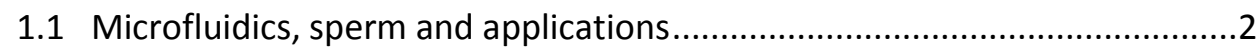

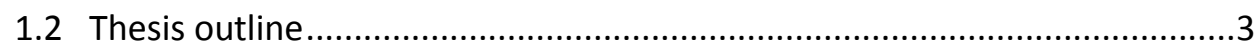

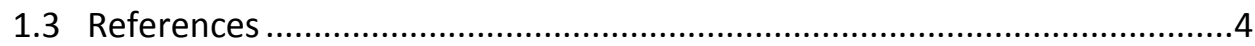

2 Single cell trapping in microfluidic systems.................................................. 7

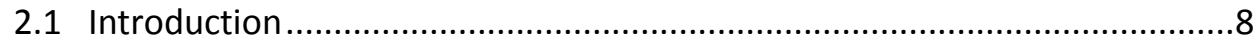

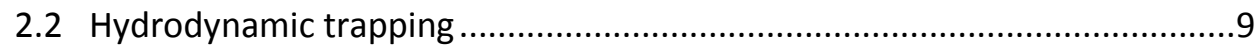

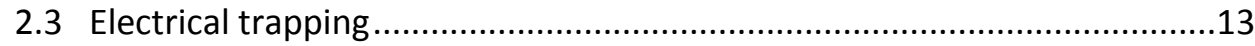

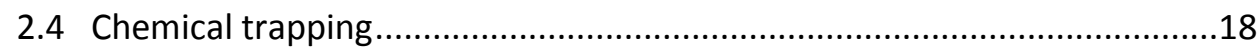

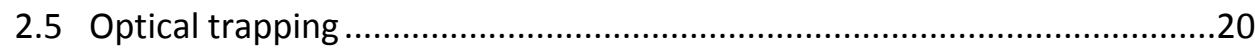

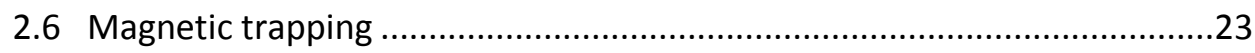

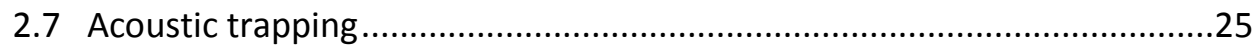

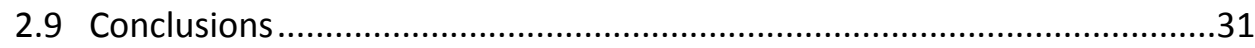

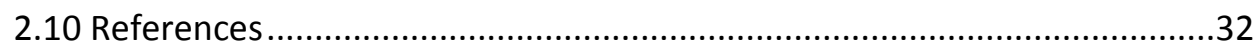

3 Electrical characterization of single cells in microfluidic systems ..................39

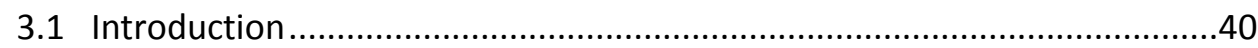

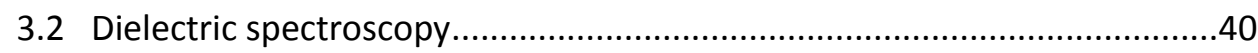

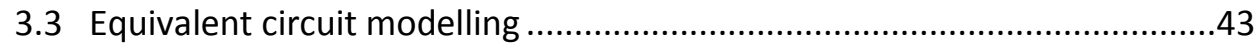

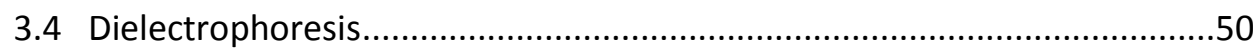

3.5 Application of microfluidic impedance cytometry .................................55

3.6 Application of microfluidic impedance spectroscopy ................................62

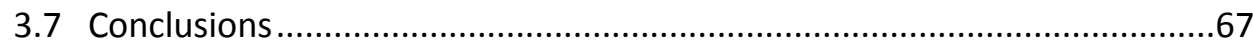

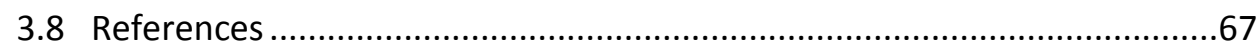

4 Microfluidic single sperm entrapment and analysis ....................................73

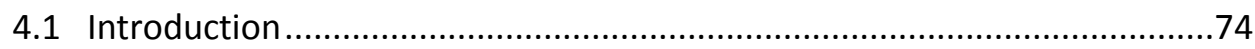

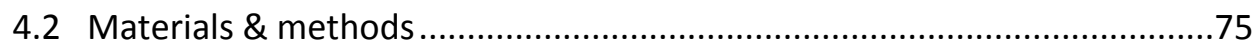

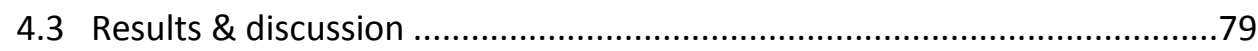

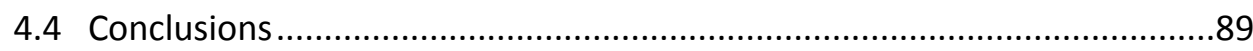

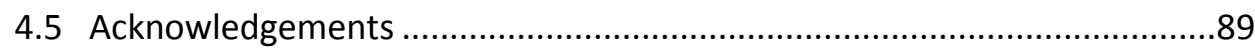

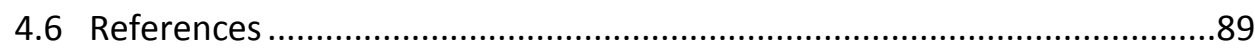

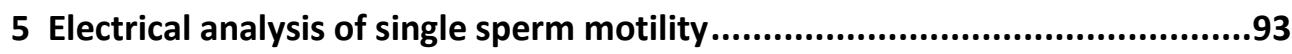

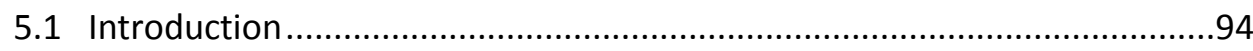

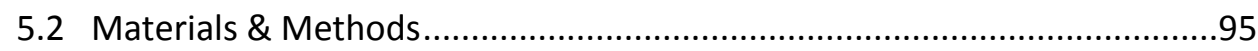




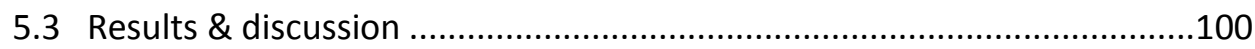

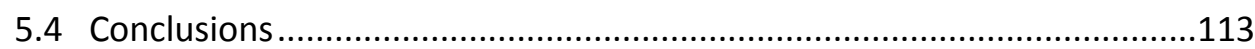

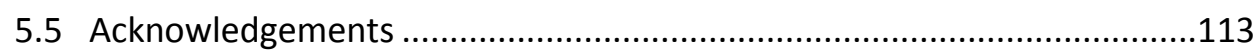

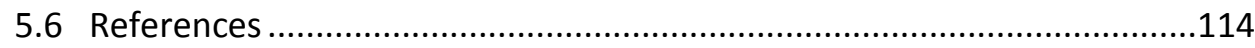

6 Towards microfluidic sperm refinement: ................................................117

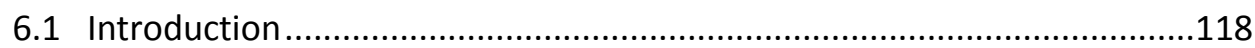

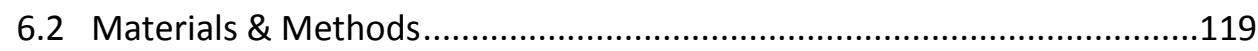

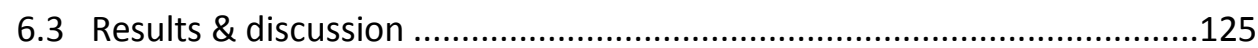

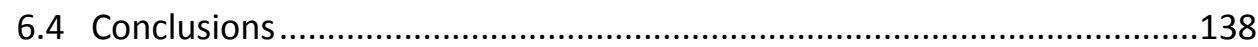

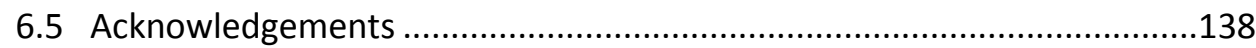

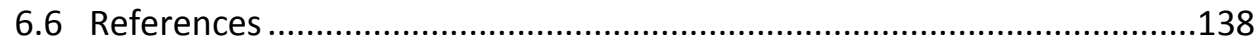

7 Sperm quality after DEP manipulation......................................................141

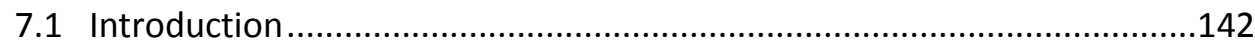

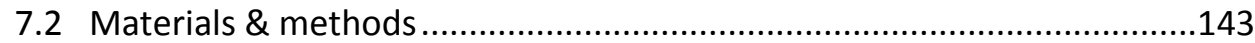

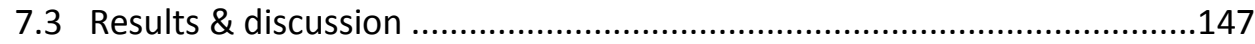

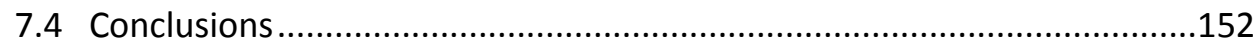

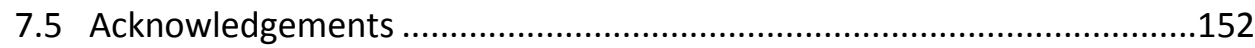

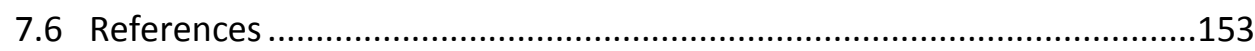

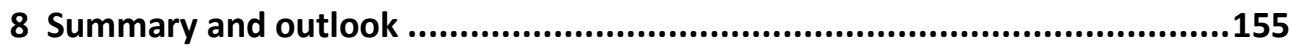

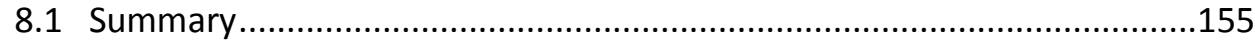

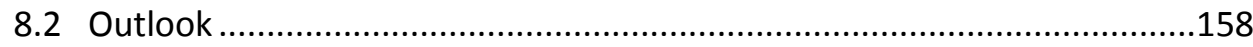

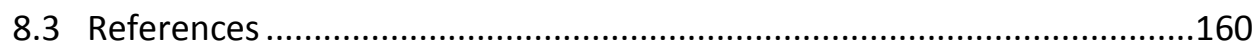

Appendix A - Fabrication of glass chips.......................................................161

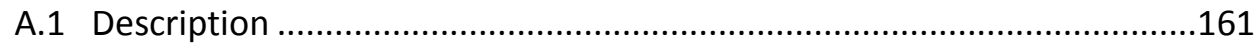

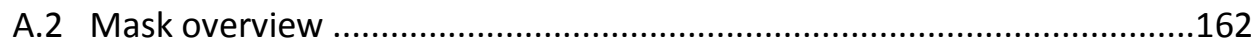

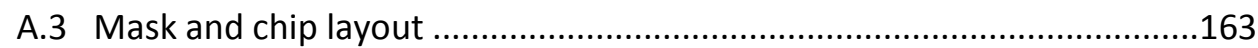

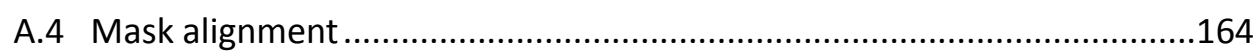

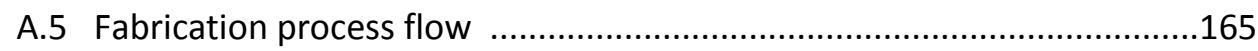

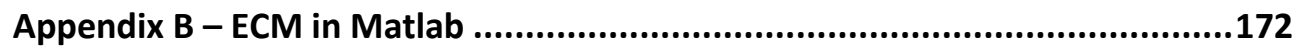

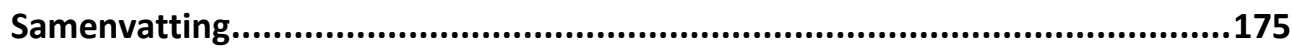

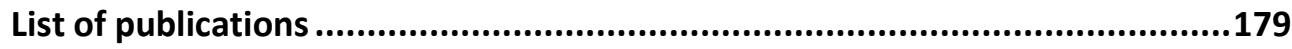

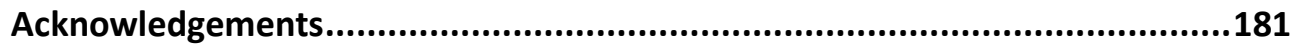




\section{Aim and outline}

This chapter introduces the aim and motivation of the work presented in this thesis. In short, this thesis reports upon the implementation of microfluidic technology for single sperm analysis. This approach might be exploited for assisted reproduction technologies (ART) in the clinic and for sperm refinement applications in the livestock industries. 


\subsection{Microfluidics, sperm and applications}

Microfluidic technology has been extensively used for a broad area of research including application in medical and forensic diagnostics [1-4]. Using this technology, small dimension microsystems can be designed, which are suitable for the analysis and manipulation of sperm cells. Since early work of Kricka et al. [5], in which a microfluidic platform was used to evaluate sperm function, microfluidic systems have been occasionally used for research on sperm. Most reports focus on the intrinsic swimming behaviour, in which sperm cells are analysed and sorted in passively driven [6, 7], flow driven [8-10] and chemo- or thermotaxis driven systems $[11,12]$. Furthermore, lens-free systems have emerged for the analysis and quantification of sperm motility $[13,14]$.

An interesting approach towards sperm analysis for clinical diagnostics was performed by Segerink et al. [15] in the BIOS Lab on a chip group at the University of Twente. They used a microfluidic chip to electrically count the amount of sperm cells in a sample by impedance cytometry, simultaneously discriminating these cells from leukocytes based on cell volume. Besides its use for cell counting applications, microfluidic impedance cytometry is a versatile method for cell analysis. It has been used for a wide range of applications including the discrimination of blood cells [16, 17] and investigation of the electrical properties of cancer cells [18-21]. Therefore, impedance cytometry might be a valuable technology for sperm research, which is still relatively unexplored.

A field, which could benefit from microfluidic technologies, is ART. Nowadays, infertility is a growing problem, affecting around $10 \%$ of all couples of reproductive age worldwide [22]. Correspondingly, the amount of ART treatments such as in vitro fertilization (IVF) and intracytoplasmic sperm injection (ICSI) have increased extensively over the last decennium. Advanced methods for sperm selection (an essential step in ART treatment) are in high demand to increase pregnancy rates. Current reports on microfluidic sperm selection, which have potential for sperm selection in ART, rely on passively driven microfluidic systems, in which high motile sperm cells are separated [6, 7]. However, these systems might not be suitable for separation of sperm cells of patients with very low sperm counts, in which sperm selection is currently performed by manual selection before ICSI procedures. This manual selection, i.e. the use of a microneedle to isolate a single sperm cell before injection in an egg cell, is currently labour-intensive and highly depends on the expertise of the clinical technician. Therefore, a microfluidic tool for advanced 
sperm selection could help the technician in sperm selection, which could improve ICIS outcome.

A different field of application, in which microfluidic technology might prove applicable, is the animal livestock industry. A nice example is reported by Segerink [23], who proposed a microfluidic chip for the detection of leukocytes in whole milk to diagnose mastitis in cows. Besides diagnostic purposes, microfluidic technology might prove useful for the refinement of sperm ejaculates, which are used in artificial insemination (Al) of animals. Refinement of sperm samples based on cell motility, viability and morphology could have a positive effect on Al outcome, e.g. the amount of piglets per litter. Driven by high interest from the livestock industry, we aim to develop a microfluidic system which can perform sperm refinement based on cell morphology.

\subsection{Thesis outline}

This thesis reports upon the use of microfluidic technology for the electrical analysis of single sperm cells. Depending on the application, single cell analysis can be performed under static conditions, in which a single cell is confined at a fixed location, or under dynamic flow conditions, flowing cells through a microfluidic channel. Chapter 2 provides a short overview upon single cell trapping techniques, which can be used to confine single cells in microfluidic systems. These techniques are based on hydrodynamic, electrical, chemical, optical, acoustical and magnetic methods. At the end of this chapter, a trapping technique is chosen for integration with an electrical analysis method. Chapter 3 reports upon the electrical properties of cells in suspension and provides a review on the electrical analysis of single cells using microfluidic impedance cytometry and microfluidic electrical impedance spectroscopy.

The two succeeding chapters focus on the entrapment of single sperm cells and electrical analysis under static conditions. In chapter 4 , a microfluidic platform is presented which is capable of single sperm trapping using a hydrodynamic trapping method. Once entrapped, sperm cells were investigated by fluorescent analysis, showing the potential to investigate the plasma membrane, acrosome integrity and sex-chromosome content of individual trapped sperm cells. A non-invasive method for single sperm analysis is reported in chapter 5 . After capturing motile sperm cells in cell traps, differential impedance analysis was performed to investigate their motility. Using this approach, the sperm cells were investigated while altering their motility by temperature changes and exposure to a chemical stimulus. 
The next two chapters report on the use of a microfluidic platform, which is designed to perform single sperm analysis under dynamic conditions. In chapter 6 , this platform was used to electrically identify cytoplasmic droplet content on sperm cells, which is a commonly occurring morphological sperm anomaly that is encountered in Al applications in the livestock industry. In order to discard these abnormal cells from the sample, which is of high interest in the livestock industry, this chapter presents a proof-of-concept, in which sperm cells and plastic beads are sorted using dielectrophoresis (DEP) after discrimination based on impedance data. To verify whether this sorting approach is safe to use and does not inflict cell damage on the sperm cells, chapter 7 reports upon the investigation of the sperm quality after exposure to electrical fields.

Finally, a summary of the presented work is given in chapter 8, after which recommendations and future research directions are discussed in the outlook.

\subsection{References}

1. Beebe, D.J., G.A. Mensing, and G.M. Walker, Physics and applications of microfluidics in biology. Annu Rev Biomed Eng, 2002. 4: p. 261-86.

2. Chin, C.D., V. Linder, and S.K. Sia, Commercialization of microfluidic pointof-care diagnostic devices. Lab Chip, 2012. 12(12): p. 2118-34.

3. Horsman, K.M., et al., Forensic DNA analysis on microfluidic devices: $A$ review. J Forensic Sci, 2007. 52(4): p. 784-99.

4. Sackmann, E.K., A.L. Fulton, and D.J. Beebe, The present and future role of microfluidics in biomedical research. Nature, 2014. 507(7491): p. 181-9.

5. Kricka, L.J., et al., Applications of a Microfabricated Device for Evaluating Sperm Function. Clin Chem, 1993. 39(9): p. 1944-47.

6. Nosrati, R., et al., Rapid selection of sperm with high DNA integrity. Lab Chip, 2014. 14(6): p. 1142-50.

7. Tasoglu, S., et al., Exhaustion of Racing Sperm in Nature-Mimicking Microfluidic Channels During Sorting. Small, 2013. 9(20): p. 3374-3384.

8. Seo, D.B., et al., Development of sorting, aligning, and orienting motile sperm using microfluidic device operated by hydrostatic pressure. Microfluid Nanofluid, 2007. 3(5): p. 561-70.

9. Lopez-Garcia, M.D.C., et al., Sperm motion in a microfluidic fertilization device. Biomed Microdevices, 2008. 10(5): p. 709-18.

10. Qiu, T., et al. A microfluidic "treadmill" for sperm selective trapping according to motility classification. in Solid-State Sensors, Actuators and Microsystems Conference (transducers), 2011 16th International. 2011. 
11. Li, Z.Q., et al., The construction of an interfacial valve-based microfluidic chip for thermotaxis evaluation of human sperm. Biomicrofluidics, 2014. 8(2): p. 024102.

12. Xie, L., et al., Integration of Sperm Motility and Chemotaxis Screening with a Microchannel-Based Device. Clin Chem, 2010. 56(8): p. 1270-8.

13. Zhang, X.H., et al., Lensless imaging for simultaneous microfluidic sperm monitoring and sorting. Lab Chip, 2011. 11(15): p. 2535-40.

14. Su, T.W., L. Xue, and A. Ozcan, High-throughput lensfree 3D tracking of human sperms reveals rare statistics of helical trajectories. Proc Natl Acad Sci USA, 2012. 109(40): p. 16018-22.

15. Segerink, L.I., et al., On-chip determination of spermatozoa concentration using electrical impedance measurements. Lab Chip, 2010. 10(8): p. 101824.

16. Spencer, D., G. Elliott, and H. Morgan, A sheath-less combined optical and impedance micro-cytometer. Lab Chip, 2014. 14(16): p. 3064-73.

17. van Berkel, C., et al., Integrated systems for rapid point of care (PoC) blood cell analysis. Lab Chip, 2011. 11(7): p. 1249-55.

18. Holmes, D., et al., Leukocyte analysis and differentiation using high speed microfluidic single cell impedance cytometry. Lab Chip, 2009. 9(20): p. 28819.

19. Kang, G., et al., Differentiation Between Normal and Cancerous Cells at the Single Cell Level Using 3-D Electrode Electrical Impedance Spectroscopy. IEEE Sens J, 2012. 12(5): p. 1084-9.

20. Kirkegaard, J., et al., Study of Paclitaxel-Treated HeLa Cells by Differential Electrical Impedance Flow Cytometry. Biosensors, 2014. 4(3): p. 257-72.

21. Zhao, Y., et al., Tumor cell characterization and classification based on cellular specific membrane capacitance and cytoplasm conductivity. Biosens Bioelectron, 2014. 57: p. 245-53.

22. Unit, W.H.O.P.o.M.a.C.H.a.F.P. Infertility : a tabulation of available data on prevalence of primary and secondary infertility. 1991.

23. Segerink, L.I., Fertility chip: a point-of-care semen analyser. 2011, University of Twente: Enschede. 
6 CHAPTER 1 


\section{Single cell trapping in microfluidic systems}

To analyse sperm cells on the single cell level, a system is required which is capable of trapping individual sperm cells. In the last two decennia, microfluidic systems have shown eminently suitable for trapping of single cells and particles. A variety of techniques is available to entrap single cells based on optical, electrical, magnetic, hydrodynamic, magnetic and acoustical methods. This chapter provides a short overview of each trapping method, including their advantages and disadvantages. Finally, the applicability of these methods is discussed for single sperm entrapment. 


\subsection{Introduction}

Investigation of cell biology on the single cell level has various benefits to routine population-based research, including the ability to investigate cellular heterogeneity. Investigation of cells on the single cell level requires an analysis platform, of which the dimensions of the analysis site are comparable to the cell itself. When one is looking for a needle in a haystack, reducing the haystack's volume will increase the odds of finding it.

Microfluidic technology is a suitable candidate for creating such a platform. After the invention of rapid prototyping of microfluidic platforms using soft lithography and poly(dimethylsiloxane) (PDMS) by the Whitesides group [4-6], the amount of research and published papers on single cell analysis grew exponentially. Generally, these platforms consist of at least a single microfluidic channel or chamber in which individual cells, suspended in (isotonic) medium, are grown on, transported to or trapped at a specific analysis site. At this site, analysis is performed by i.a. optical (e.g. fluorescence, Raman spectroscopy), electrical (e.g. amperometry, impedance spectroscopy, electrorotation) or mechanical (e.g. micromanipulation) means under static or dynamic flow conditions [1, 7]. For some of these methods, individual cells have to be confined to a fixed position with respect to the analysis site during the analysis.

One of the aims of this project is to perform electrical analysis on individual sperm cells using impedance spectroscopy (paragraph 3.1.7). Since spectroscopic analysis over a broad frequency range can depend on measurement times in the order of seconds, individual cells need to be trapped during analysis. Therefore, this chapter provides a short overview of available techniques for single cell trapping. These techniques are based on optical, electrical, magnetic, hydrodynamic, magnetic and acoustical methods [1-3]. The main emphasis of this overview lies on hydrodynamic and electrical trapping procedures. At the end of each paragraph, a short summary of the design considerations is provided, elaborating on the advantages and disadvantages of each method concerning trapping force, throughput, inflicted cell damage, fabrication and setup complexity.

In order to select the best trapping method to confine individual sperm cells, the final paragraph provides a summary of the current papers on sperm trapping. Furthermore, the applicability of the presented trapping methods is discussed towards integration with non-invasive analysis using impedance spectroscopy. 


\subsection{Hydrodynamic trapping}

A straightforward way to entrap cells is the use of hydrodynamics. Microfluidic systems for hydrodynamic cell trapping contain small microchannels or microstructures, which allow the flow of fluid but restrict the cell passage and thus act as cell traps. Cell trapping can be easily performed by controlling the fluid flow through or pressure over a cell trap.

\subsubsection{Dam or sieve-like traps}

A variety of hydrodynamic cell traps have been reported over the years. A good example is the dam-like cell trap reported by Yang et al. [8] (Fig. 2-1A). In a parallel channel setup, two channels were separated by a dam structure, which height was $5 \mu \mathrm{m}$ smaller than the total channel height, allowing fluid transport between the two channels. By controlling the flow rates in both channels, a pressure difference was induced resulting in the entrapment of up to 20-30 HL-60 cells. Subsequently, ATP dependent calcium uptake was investigated in individually trapped cells. Valero et al. [9] reported the use of a sieve structure with $3 \mu \mathrm{m}$ orthogonal side channels to entrap up to 7 individual HL-60 cells. Under spatial confinement, apoptosis of HL-60 cells was studied. A similar geometry was used in a report of Zhu et al. [10] to entrap up to 10 single yeast cells and study them by electrical impedance spectroscopy using integrated microelectrodes. Correspondingly, Long et al. [11] designed a microfluidic system containing two main channels, which are interconnected by $20 \mu \mathrm{m}$ long and $1.1 \mu \mathrm{m}$ high side channels. In 600 parallel side channels, bacteria were trapped, grown and analysed. Based on this channel geometry, de Wagenaar et al. [12] reported upon the entrapment of individual sperm cells in $2 \mu \mathrm{m}$ wide and $1 \mu \mathrm{m}$ high side channels. After entrapment, sperm cell viability, the integrity of the acrosomal membrane and the sex-chromosome content was investigated.

Previous reports showed the potential of planar hydrodynamic cell trapping, applying a trapping force on the cells in parallel with respect to the substrate surface. Various reports show the use of engineered microcavities to entrap single cells by an out of plane trapping force. Osada et al. [13] showed the use of $3 \mu \mathrm{m}$ wide micro cavities (>100k per chip) in a poly(ethyleneterephthalate) substrate to entrap cells by applying a negative pressure between the top and bottom compartment with respect to the substrate. These cells were patterned and cultured on a glass slide after transfer printing them using solidified agarose. In a report of Han et al. [14] $2 \mu \mathrm{m}$ wide microcavities in a silicon oxide membrane were 
used to entrap chromaffin cells in microwells, fabricated in polyimide. Integrated gold electrodes were used to investigate ion-channel activity using impedance spectroscopy. A similar approach was reported by Swennenhuis et al. [15] (Fig. 2-1B). They used a $1 \mu \mathrm{m}$ thick silicon nitride membrane with $5 \mu \mathrm{m}$ pores to entrap cells of three different cell lines (SKBR-3, LNCaP and PC3) in $70 \mu \mathrm{m}$ wide and 360 $\mu \mathrm{m}$ deep microwells ( 6400 wells per chip). Individual cells could be easily recovered by punching the membrane of a single well, hereby moving the cell to the bottom compartment. This device was used to trap MCF-7 cancer cells from leukocyte depleted, MCF-7 spiked whole blood and to perform cell lysis and DNA amplification on EpCAM-stained MCF-7 cells [16].

\subsubsection{Single channel crossflow trapping}

In previous examples, cell trapping was accomplished by using microstructures, which separated two distinct microfluidic channels or chambers. The relative pressure over and fluid flow between the separate channels or chambers were used to control cell entrapment. Various reports show the use of a single microfluidic channel with a single channel inlet and outlet for individual cell entrapment. A good example is the report of Tan et al. [17]. They presented a device containing a meander-shaped main channel, in which small intersections were designed to form short-cuts within the channel (Fig. 2-1C). Since these short-cuts have a lower flow resistance, gas bubbles were dragged into the short-cut, hereby trapping them. As a result, all 100 trapping sites were filled sequentially. This design was optimized by Kobel et al. [18] for efficient cell trapping (97\%) and long-term culture of up to 400 trapped EG7 cells. The same principle was used to entrap HeLa cells and to investigate the effects of anti-cancer drugs on the single cell level by Wang et al. [19]. A similar, sequential trapping platform is reported by Sochol et al. [20]. In their research, they used a meander-shaped main channel (Fig. 2-1D), which was interconnected by 100 trapping sites in a row, yielding sequential and reversal trapping of polystyrene beads and endothelial cells with an efficiency of $99.8 \%$ and $78.0 \%$, respectively.

\subsubsection{Micropost arrays}

The previous described reports have shown cell entrapment in between separate channels or chambers. Cell entrapment can also be achieved by using integrated traps in a single microfluidic channels. Di Carlo et al. [21, 22] used large arrays of microposts (Fig. 2-1E) for cell trapping with an array density of $3300 \mathrm{traps} \cdot \mathrm{mm}^{-2}$, yielding approximately 400 traps per device. These posts were designed with a 
recess etch, ranging from 10 to $60 \mu \mathrm{m}$ in height, to capture single cells or small groups of cells.
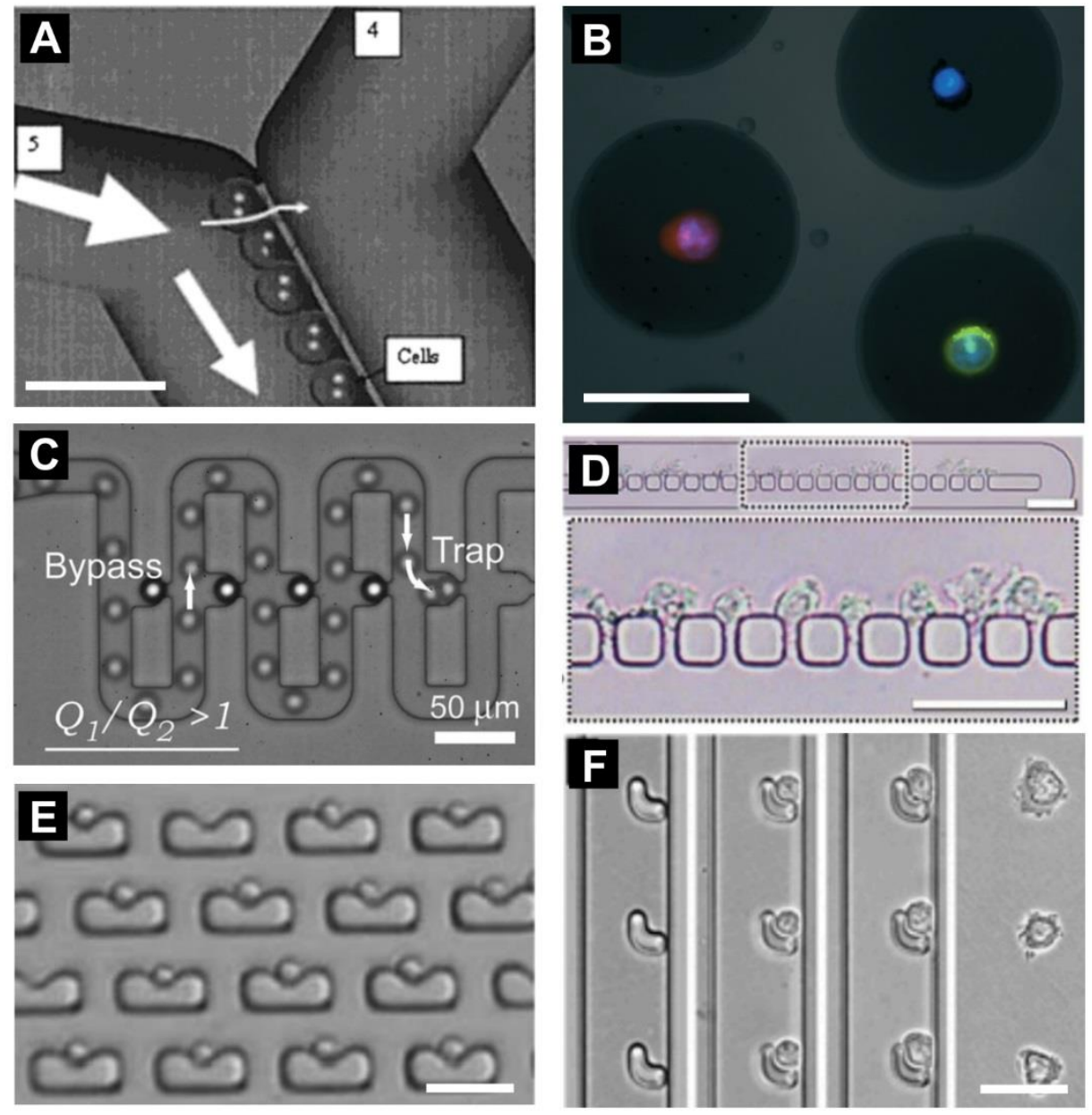

Fig. 2-1: Cell trapping by hydrodynamic procedures. Dam and sieve-like structures were used by A) Yang et al. [8] and B) Swennenhuis et al. [15]. Single channel cross-flow type devices were utilized by C) Tan et al. [17] and D) Sochol et al. [20]. Micropost arrays for cell entrapment were developed by E) Di Carlo et al. [21, 22] and F) Zhang et al. [24]. All scale bars are $50 \mu \mathrm{m}$. (All images are adapted from the indicated references). 
These arrays were used for single HL-60 cell perfusion culture experiments and for investigation of intracellular carboxylesterase kinetics and concentration in Jurkat, HeLa and T2932 cells lines by fluorescence microscopy [21].

A similar approach for cell entrapment was reported by Skelley et al. [23] using a device with an array density of approximately 6000 traps per $32 \mathrm{~mm}^{2}$. Besides cell entrapment by forward-flowing cells in the recess-etched region of the micro posts, a second and deeper recess at the backside of the cell traps was used for trapping of a second cell type with reversed flow. Successful cell pairing experiments were performed with fibroblasts, mouse embryonic stem cells and myeloma cells, yielding pairing efficiencies up to $70 \%$. Furthermore, high throughput cell fusion experiments were performed by means of chemical and electrical stimuli. In a comparable device, Kemna et al. [25] showed cell pairing and electrofusion of individual human B cells with mouse myeloma cells to generate functional hybridomas. They reported electrofusion efficiencies up to $1.2 \%$, which was reported as an improvement with respect to conventional bulk electrofusion methods. A recent report of array-based single cell trapping is reported by Zhang et al. [24]. They used microtraps, which were closely positioned to the channel wall, to entrap cells in a high throughput manner (Fig. 2-1F). After cell entrapment and adhesion, the microfluidic top-layer was removed, allowing the cells to grow freely. Heterotypic cell pairs were created by trapping two different cell types in neighbouring cell traps, which were facing in opposite directions. After trapping and adhesion of the first cell type, the flow was reversed to trap the second cell type [24].

\subsubsection{Design considerations}

Hydrodynamic cell traps are usually simple to operate and only require a syringe or pressure pump to create fluid flows for cell entrapment, avoiding the need for expensive instrumentation. Therefore, the throughput of hydrodynamic trapping assays is easier to increase compared to electrical, optical, magnetic and acoustical traps. Complex fabrication of multi-layered molds to create microfluidic devices could be a drawback of this method. Furthermore, once a cell trap is optimized for a certain cell size, a slight variation in application requires new device fabrication. Additionally, cell deformability and stickiness are known factors, which contribute to channel clogging and negatively affect trap reversibility. 


\subsection{Electrical trapping}

Electrical trapping of particles and cells can be performed using DEP. This phenomenon describes the force, which is the result of a field-induced dipole moment on a dielectric, polarizable particle when introduced to a non-uniform electric field [26]. When this dipole moment arises from the accumulation of charge on the particle-electrolyte interface, it is called interfacial polarization. Theoretical description and calculation of the DEP phenomenon are found in paragraph 3.5.

A large amount of papers have shown the applications of DEP for cell filtration, concentration, separation and entrapment in microfluidic systems [27-29]. In general, DEP in microsystems is employed by applying an alternating current (AC) to integrated microelectrodes, thereby creating an inhomogeneous electrical field, which manipulates particles and cells by repulsion from (negative DEP, or nDEP) or attraction to (positive DEP, or pDEP) high electrical field gradients. The following paragraphs will focus on DEP platforms for the entrapment of single cells. Roughly, these platforms can be classified into three categories: nDEP-based, pDEP-based and insulator-based DEP (iDEP) cell traps.

\subsection{1 nDEP-based traps}

All nDEP traps are based on applying repulsive forces on a cell to achieve spatial control. Various electrode configurations for single cell entrapment have been reported, including planar configuration, multi-layer configuration and 3Dconfiguration.

\section{Planar configuration}

Planar electrode configuration consists of multiple electrodes, which are positioned on the same plane. Compared to multi-layer and 3D-configurations, planar configurations are the most simple to fabricate.

An early report of single particle trapping is published by Hughes and Morgan [30]. They used quadrupole electrodes, in which 4 electrodes are arranged in a cross-like fashion to create a local field minimum in the centre of the electrode array, which is used for particle entrapment (Fig. 2-2A). Using this electrode array, they showed nDEP entrapment of single plastic spheres, capsids and virus particles ranging from 93-250 nm in size (typically at $15 \mathrm{MHz}, 2.5 \mathrm{~V}$ ). In a follow-up paper, they showed the ability to separate micron-sized particles based on differences in dielectric properties [31]. Similarly, Voldman et al. [31] reported the use of a quadrupole nDEP trap for particle entrapment (Fig. 2-2B). They elaborately investigated the 
hydrodynamic drag forces and DEP trapping forces acting on entrapped polystyrene beads to optimize the trapping efficiency.

The group of Voldman also reported on nDEP cell trapping using a different planar electrode geometry. They designed [32], modelled [33] and investigated nDEP microwells for the entrapment of single cells [34]. These nDEP microwells, which consisted of square and line electrode geometries and yielded an attractive force of approximately $1 \mathrm{pN}$ at $0.5 \mathrm{~V}$, allowed entrapment of both adherent (HeLa) and non-adherent (HL-60) cell types in parallel fashion (Fig. 2-2C). Unaffected cell viability and proliferation were observed over 4 days during entrapment $(10 \mathrm{MHz}$, $0.5 \mathrm{~V})$.

A combination of similar microwell traps and quadrupole electrodes was reported by Jang et al. [35] The quadrupole electrodes were used to induce bulk motion of HeLa cells towards the microwell electrodes ( $5 \mathrm{MHz}, 4-5 \mathrm{~V})$, whereas the microwell electrodes were used to entrap individual cells ( $5 \mathrm{MHz}, 3 \mathrm{~V}$ ) generating a DEP force up to $4 \mathrm{pN}$. In follow-up research, these quadrupole electrodes were combined with ring-shaped electrodes [36] and line-shaped electrodes [37] for single cell entrapment and impedance analysis.

\section{Multi-layer configuration}

In multi-layer configurations, electrodes are positioned on two or more different planes, e.g. on the top and bottom of a microfluidic channel. A top-down quadrupole nDEP cage for single cell trapping and manipulation was used by Lombardini et al. [38]. Polystyrene particles and $\mathrm{K} 562$ cells were successfully positioned and levitated in microwells by $\mathrm{nDEP}(600 \mathrm{kHz}, 4-6 \mathrm{~V})$. In a different report from the same group, multi-layer electrodes were used to create vertical nDEP traps in engineered microwells [42]. A ring-shaped trap was designed by Thomas et al. [39]. Their device consisted of two platinum rings separated by a dielectric layer (Fig. 2-2D). Using their trap, latex particles $(15.6 \mu \mathrm{m})$ and HeLa cells were attracted with a trapping force of 23 and $13.8 \mathrm{pN}$, respectively $(1 \mathrm{MHz}, 2.5 \mathrm{~V})$.

An octopole electrode system was reported by Schnelle et al. [40, 43]. They used two planar quadruple systems on top of each other to entrap particles and cells in the middle of the trap (Fig. 2-2E). Using this platform, single mouse hybridoma cells were entrapped ( $250 \mathrm{MHz}, 5 \mathrm{~V}$ ). A similar electrode geometry is reported by Fuhr et al. [44] for the entrapment of single sperm cells ( $8 \mathrm{MHz}, 2.5 \mathrm{~V})$. In the same report, they showed parallel entrapment of single, motile sperm cells in an interdigitated electrode system (5 MHz, $2.5 \mathrm{~V}$ ). 

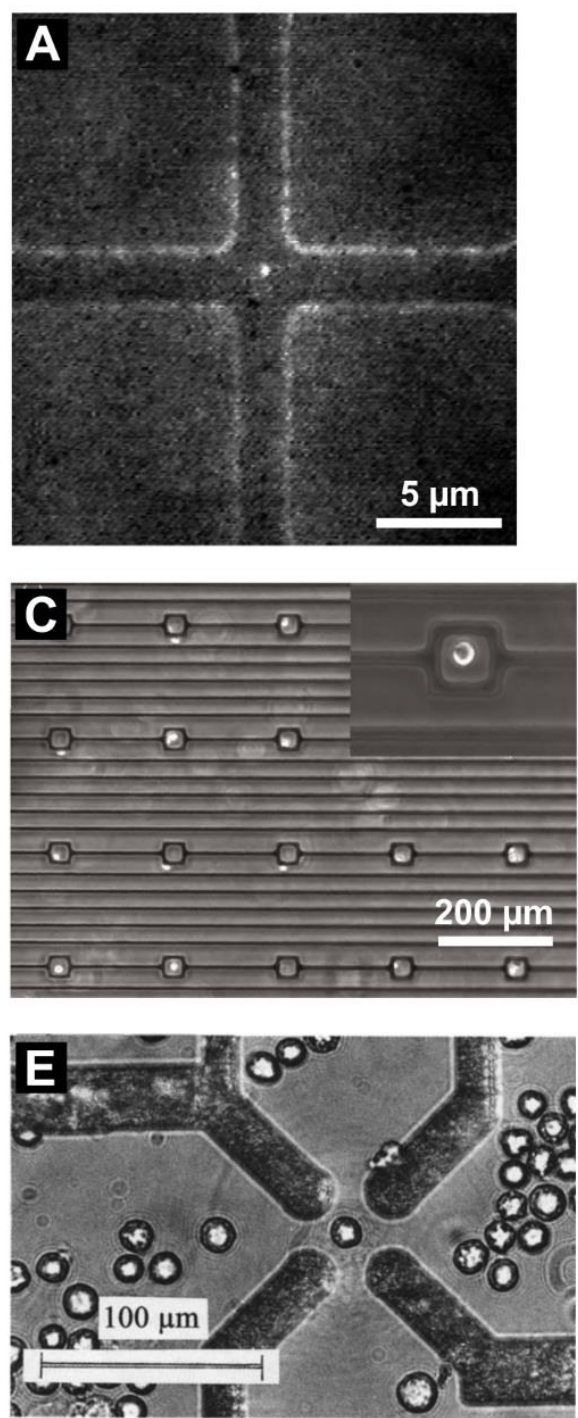
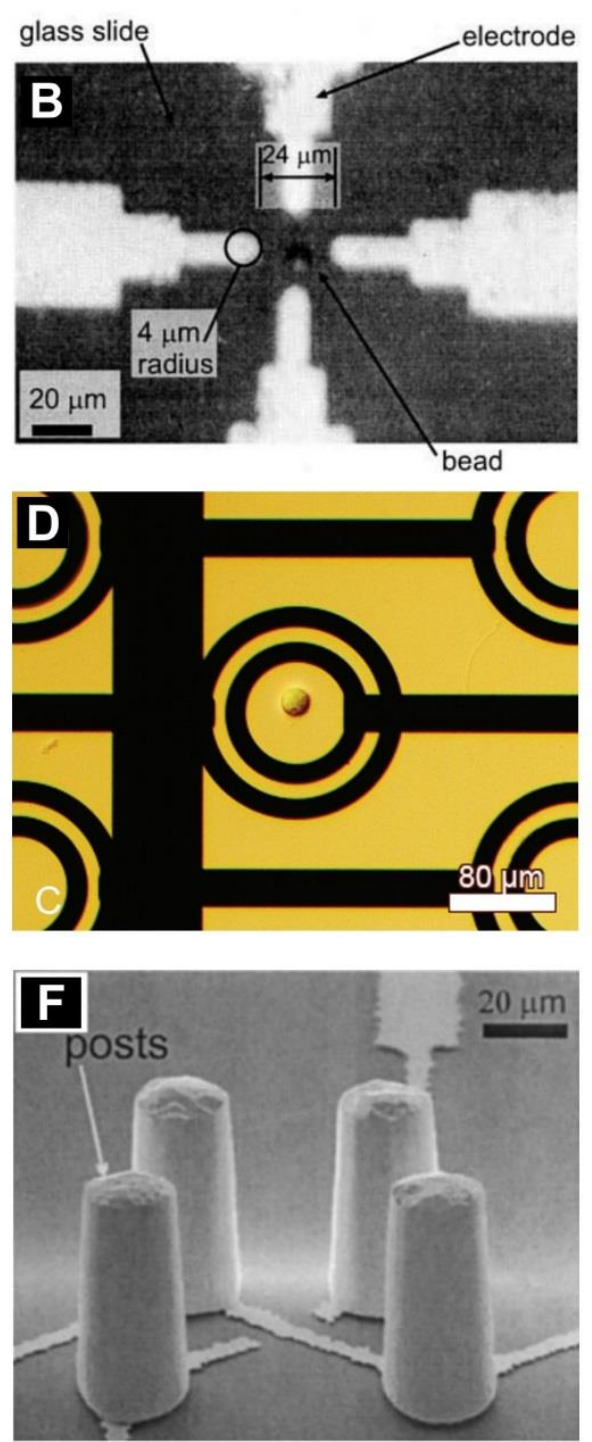

Fig. 2-2: Cell trapping by nDEP. Planar quadrupole configurations were used by A) Hughes and Morgan [30] and B) Voldman et al. [31]. C) A single-layer and D) multi-layer nDEP trap were reported by Mittal et al. [34] and Thomas et al. [39]. E) A top-down octopole orientation was reported by Schnelle et al. [40] F) A 3D (extruded) quadrupole orientation was used by Voldman et al. [41] (All images are adapted from the indicated references). 


\section{D-configuration}

A 3D nDEP trap consists of 3D-fabricated microelectrodes. Voldman et al. [41, 45] reported upon the design of an array of gold cylindrical electrodes (Fig. 2-2F) in a quadrupole orientation. These traps showed a holding force of $>100 \mathrm{pN}$ on beads (1 $\mathrm{MHz}, 3 \mathrm{~V}, \varnothing=13.2 \mu \mathrm{m}$ ). Furthermore, they showed entrapment of HL-60 cells using a holding force of $\sim 40 \mathrm{pN}$ while retaining the induced transmembrane potential at $30 \mathrm{mV}$.

\subsection{2 pDEP-based traps}

Although pDEP traps are easier to engineer and fabricate compared to nDEP traps, they are not often used for (single) cell entrapment. Low-conductive buffers are required for pDEP manipulation, which disturb normal cell physiology [26]. Furthermore, direct electrode-cell contact using pDEP traps can damage cell membranes [26].

Despite these drawbacks, several reports showed pDEP trapping of single cells. Suehiro and Pethig [46] used a grid electrode system consisting of parallel strip electrodes for pDEP trapping of protoplasts $\left(10 \mathrm{kHz}-10 \mathrm{MHz}, 2.8 \mathrm{~V}, \sigma_{\text {medium }}=0.1\right.$ $\left.\mathrm{mS} \cdot \mathrm{m}^{-1}\right)$. By addressing different strip electrodes, the position of these cells could be controlled.

Qian et al. [47] reported upon a planar concentric ring levitator, in which pDEP was used to levitate protoplasts above the electrode surface by counteracting the gravitational force. Gray et al. [48] produced a device with large arrays of pDEP traps, which are aligned with adhesive fibronectin-coated regions (Fig. 2-3A). After DEP trapping of bovine endothelial cells $\left(2 \mathrm{MHz}, 2.5 \mathrm{~V}, \sigma_{\text {medium }}=20 \mathrm{mS} \cdot \mathrm{m}^{-1}\right)$, the cells started to spread on the adhesive regions $1 \mathrm{~h}$ after trapping. The cell viability drastically decreased when increasing the applied amplitude to $10 \mathrm{~V}$. Another example of an array of pDEP traps was reported by Taff and Voldman [49]. These traps consisted of two electrodes on two different layers, separated by a dielectric layer, which contained a tiny aperture (Fig. 2-3B). Silver-coated beads ( $\varnothing=20 \mu \mathrm{m})$ and HL-60 cells were trapped ( $1 \mathrm{MHz}, 2 \mathrm{~V}$ ), achieving holding forces between 240 $430 \mathrm{pN}$. Furthermore, particles and cells could be selectively separated by addressing individual electrodes.

\subsection{3 iDEP-based traps}

A relatively new method of DEP trapping of particles and cells is iDEP. By applying an $A C$ or DC potential over a channel containing insulating structures, local nonuniform fields are created, which either attract or repulse cells. Since external 
electrodes are used for iDEP manipulation, no microelectrodes are necessary, hereby simplifying device fabrication.

Bhattacharaya et al. [50] used iDEP for single cell entrapment. A single MCF-7 cancer cell could be entrapped near the microstructures by a generated pDEP force after applying a potential of $+100 \mathrm{~V}$ at the channel inlet, $-100 \mathrm{~V}$ at the channel outlet and $+100 \mathrm{~V}$ at the side channels (Fig. 2-3C). In follow-up research, they showed the ability to selectively entrap MCF-7 cells from a mixture of both mammalian peripheral blood mononuclear cells and a second breast cancer cell type [51]. They suggest that discrimination is based on differences in membrane properties of both cancer cell lines.

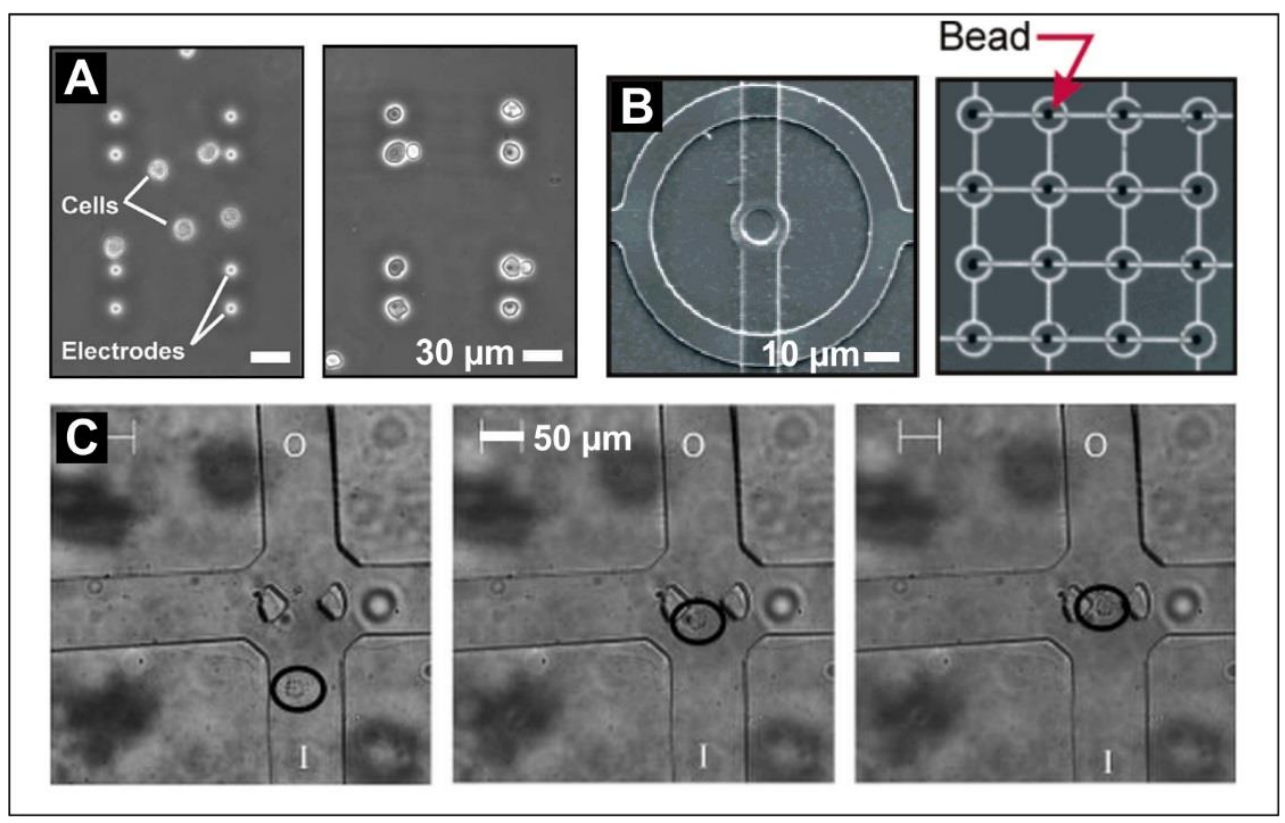

Fig. 2-3: Cell trapping by pDEP and iDEP. A) Gray et al. [48] entrapped bovine endothelial cells on a fibronectin-coated surface using pDEP. B) Taff and Voldman [49] used a pDEP platform to entrap and spatially manipulate HL-60 cells. C) iDEP trapping was used to entrap a single MCF-7 cell by Bhattacharya et al. [50] (All images are adapted from the indicated references). 


\subsubsection{Design considerations}

All described DEP techniques have advantages and disadvantages when applied for single cell trapping in microfluidic systems. In terms of fabrication, pDEP systems are most easy to fabricate. Using simple planar microelectrodes, cells can be attracted to high field gradients, achieving higher holding forces compared to nDEP systems. However, due to the use of low conductive buffers and direct cellelectrode contact, cells are more readily damaged in pDEP systems. Contrarily, nDEP systems allow use of high conductive saline solutions, in which cells are repulsed from the electrode surface, hereby minimizing cell damage. A disadvantage of cell repulsion is the need for a 3D-confinement to achieve moderate holding forces, increasing the complexity of device fabrication. Furthermore, increasing the holding forces in nDEP systems by increasing the field potential, can result in high fluid temperatures due to Joule heating. This could have a harmful effect on entrapped cells. When using iDEP for cell entrapment, the integrated microstructures induce the DEP effect. Therefore, no microelectrodes are necessary, decreasing the fabrication complexity and costs which could facilitate mass production of DEP-based microfluidic systems [52]. However, high voltage excitation may result in high transmembrane potentials, which could lead to cell damage. The effect of Joule heating and transmembrane potential have to be considered when manipulating cells in microfluidic systems.

\subsection{Chemical trapping}

Chemical trapping is based on the active binding of cells to adhesive micropatterns $[2,53]$. In micro contact printing $(\mu \mathrm{CP})$ a master mold, containing topographical microstructures, is used to create elastomeric stamps (Fig. 2-4A) [53]. These structures are coated with a (bio)molecule solution and transferred to a substrate to create micropatterns (Fig. 2-4B). Traditionally, master molds are created using photolithographic techniques although alternatives are available. The stamps are usually fabricated using PDMS, however materials such as poly-olefin-plastomers and agarose are suitable as well [53]. Adhesive micropatterns are made using extracellular matrix proteins or synthetic peptide constructs to promote cell adhesion. Examples are fibronectin, laminin and collagen for adhesion of endothelial cells, neuronal cells and mesenchymal stem cells, respectively [53]. To restrict cell patterning to the predefined regions, a cell-repellent compound is used to backfill the unpatterned surfaces. Commonly used surface passivation agents are poly-L-lysine grafted poly(ethylene glycol) (PLL-g-PEG), pluronic acid and bovine serum albumin (BSA) [53]. 


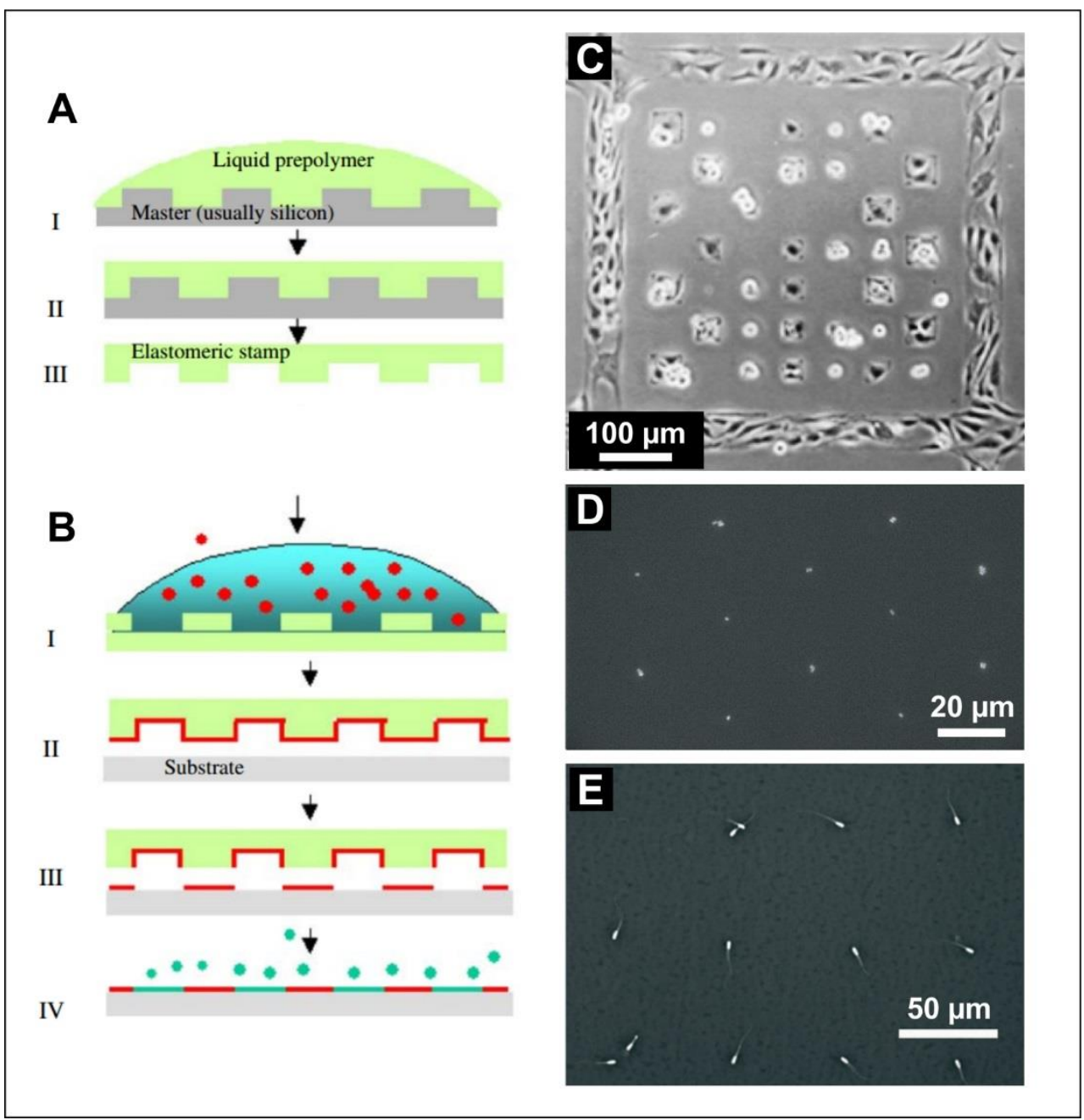

Fig. 2-4: Microcontact and microfluidic patterning. A) Master molds for patterning are usually fabricated by photolithographic techniques [53]. An elastomeric mold is prepared by pouring a liquid prepolymer over the master mold, which is ready for patterning after curing. B) A solution of (bio)molecules is used to ink the stamp (I-II), which is subsequently transferred to a substrate (III). Unpatterned regions are backfilled (IV) using a cell-repellent compound. Single cell trapping of C) bovine capillary endothelial cells by Kane et al. [54], D) E-Coli by Xu et al. [55] and E) sperm cells by Frimat et al. [56] (All images are adapted from the indicated references). 
The field of single cell patterning is pioneered by Whitesides and Ingber [54, 57-59]. They showed single cell patterning on self-assembled monolayers (SAMs) of alkanethiols on gold surfaces. Proteins and cells can bind to the SAM regions containing adhesive alkanethiol, whereas no bonding is taking place on the nonadhesive alkanethiol-coated regions. Using fibronectin-functionalized SAMs, they comprehensively studied the effects of pattern size and geometry on cell growth [57], spreading [59], apoptosis [57] and differentiation [58] of endothelial cells.

$\mu \mathrm{CP}$ has been exploited for studying the effects of geometrical constrains on differentiation of human mesenchymal stem cells (Fig. 2-4C) [60,61]. These studies showed that cell shape regulates the differentiation to an osteogenic or adipogenic lineage. Furthermore, $\mu \mathrm{CP}$ has been used to create arrays of bacteria on agarose for high throughput studies (Fig. 2-4D) [55, 62]. In a recent report of Frimat et al. [56], single sperm cells were entrapped using fibronectin spots (Fig. 2-4E). After entrapment, the motility of individual sperm cells was investigated.

Various $\mu \mathrm{CP}$ applications and modified procedures have been reported in literature. A comprehensive review of $\mu \mathrm{CP}$ is reported by Falconnet et al. [53].

\subsubsection{Design considerations}

$\mu \mathrm{CP}$ is a non-invasive technique, which has been used for the entrapment of individual, adherent cells. The holding forces of this technique are small and depend on direct contact between cell and patterned substrate. However, after cell adherence, these forces extensively increase due to bonding of cell integrins to patterned proteins. Fabrication of the master molds and PDMS stamps is relatively easy, although transfer printing of cell-adhesive compound (e.g. fibronectin) is a manual process which is hard to standardize. As a consequence, the smallest structure size which can be printed using PDMS stamps, is in the order of $1 \mu \mathrm{m}$. Furthermore, homogenous patterns can only be fabricated in reproducible fashion using stamps with a limited pattern size $\left(<2 \times 2 \mathrm{~cm}^{2}\right)$ [53].

\subsection{Optical trapping}

Optical trapping is performed by using optical tweezers (OTs). OTs use highly focused laser beams which can be used to entrap particles and cells by attracting them to a higher optical field gradient (Fig. 2-5A). When focusing a laser on an object, two forces affect the object: the scattering force and the gradient force. The scattering force pushes the object in the propagation direction of the laser beam, whereas the gradient force moves the object towards high field gradients due to 
the object's dipole moment. Depending on the size of the object with respect to the applied optical wavelength, this phenomenon is explained differently [63, 64]. More comprehensive literature on the theoretical aspects of optical trapping can be found in a review by Jonas and Zemanek [65].

A wide variety of studies have employed optical trapping for single cell analysis, studying mechanical and electrical properties of erythrocytes, single cell poration, biomolecule injection, cell fusion and integration with Raman spectroscopy [63, 64, 68]. An interesting example is presented in the report of Lim et al. [66]. They showed cell deformation of individual red blood cells using two non-specifically attached silica microbeads ( $\varnothing=4.12 \mu \mathrm{m}$ ), of which one was fixated to a moving glass chamber and the other was held into place using an OT (Fig. 2-5B). Cell deformation was studied using forces up to $340 \mathrm{pN}$.

Integration of optical manipulation in microfluidic systems yielded a rapidly emerging area of research called optofluidics [69], combining accurate control of cell position with extensive control over cell surroundings. In microfluidic systems, numerous reports show the capability of cell trapping, transportation, sorting (of blood cells), cell poration and/or biomolecule insertion and force measurements [63]. Several recent publications show the integration of OTs with single cell analysis techniques in microfluidic systems, including Raman [70, 71] and fluorescence spectroscopy $[72,73]$. An illustrative example, showing the potential of OTs in microfluidic systems, is reported by Eriksson et al. [67]. They used an OT to capture, transport and pattern individual yeast cells within a microfluidic channel. After patterning yeast cells with medium sized buds in columns 1, 3 and 5, and cells without/with a small bud in column 2 and 4 (Fig. 2-5C), the cellular response was studied as a function of reversible changes in glucose concentration.

An interesting application of optical tweezers is the entrapment of single sperm cells [74, 75]. Ohta et al. [75] showed the use of an OT to inflict optically-induced DEP forces on entrapped sperm cells. Using these induced forces, the motility of sperm towards a moving optical field was investigated, showing differences in viable and non-viable sperm cells. Furthermore, Nascimento et al. used OTs to investigate the swimming forces of dog [74] and primate sperm [76]. Furthermore, they investigated the effects of optical trapping on the sperm motility. Trapping sperm cells for 5-10 s did not significantly affect the sperm motility, although a trapping duration of $15 \mathrm{~s}$ at a laser power of $420 \mathrm{~mW}$ resulted in a decrease of sperm curvilinear velocity after entrapment [74]. 


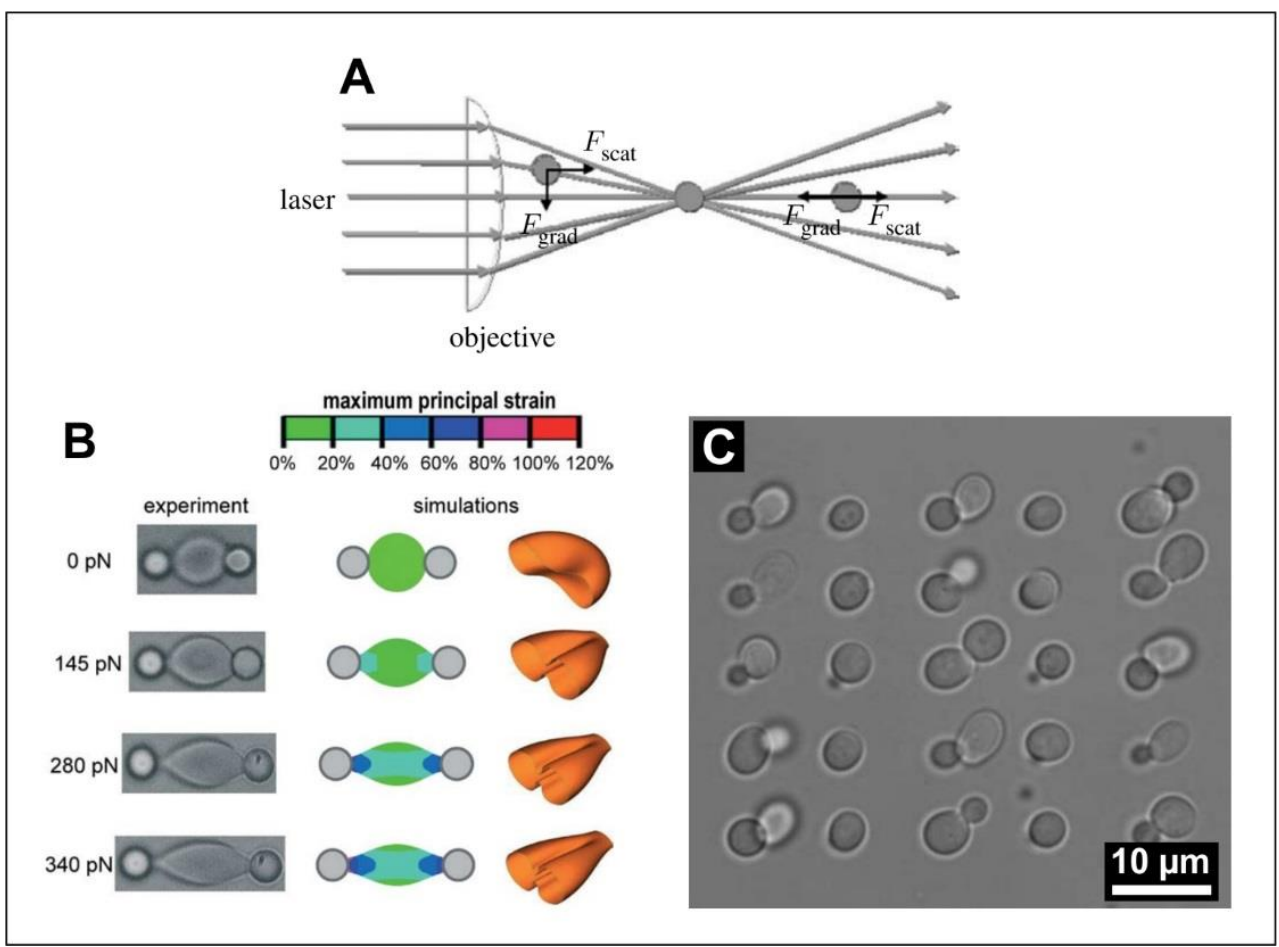

Fig. 2-5: Optical tweezers use highly focused laser beams for entrapment and manipulation of particles and cells. A) Upon beam incidence, a scattering and gradient force act on the object [63]. Optical tweezers have been used for a wide range of applications, e.g. B) for the analysis of red blood cell deformability performed by Lim et al. [66] and C) for capture, transport and patterning of yeast cells in a microfluidic channel by Eriksson et al. [67] (All images are adapted from the indicated references).

\subsubsection{Design considerations}

OTs are very suitable for accurate control and manipulation of single cells. High powered lasers can induce trapping forces on cells in the range of 100-1000 pN [3], yielding stable cell entrapment under fluid flow conditions. However, photo induced cell damage limits the use of optical tweezers [71]. Photo damage is caused by fluid heating or the creation of radical oxygen species, which are known to extensively damage biological cells $[63,71]$. Although some reports have shown long-term trapping of cells without significant cell damage, the applied optical frequency and intensity must be considered attentively to minimize induced cell damage. Additional disadvantages of optical traps are high involved costs and complexity of the required setup. Furthermore, the throughput of optical trapping systems is low and their upscaling potential is limited. 


\subsection{Magnetic trapping}

Magnetic trapping is based on the intrinsic or extrinsic magnetic properties of cells [89]. Intrinsic magnetic behaviour can be caused by iron-containing structures such as iron-containing haemoglobin in erythrocytes. Extrinsic magnetic properties can be induced by immunolabeling the cells with magnetic nanoparticles (MNPs). Various microfluidic-based approaches show the use of intrinsic (e.g. separation of RBCs and white blood cells [77]) and extrinsic magnetic properties (e.g. isolation immunomagnetically-labelled circulating tumour cells [78]) for cell separation applications. Several reports show the use of magnetic systems for single cell entrapment. In microfluidic systems, magnetization is established by three different methods: the use of integrated permanent magnets, microelectromagnets or externally applied magnetic fields.

\subsubsection{Integrated permanent magnets}

Few reports have shown the use of integrated permanent magnets for single cell entrapment. A disadvantage of permanent magnets is the decrease of field strength with decreasing magnet size, yielding small magnetic fields when scaling-down for single cell applications. Winkleman et al. [79] showed the entrapment of polystyrene spheres, fibroblasts, yeast and algae using a 3D magnetic trap with permanent magnets in an aqueous solution of paramagnetic ions (Fig. 2-6A). They calculated a trapping force of $5.5 \mathrm{pN}$ on a diamagnetic sphere with a radius of 2.5 $\mu \mathrm{m}$.

\subsubsection{Micro-electromagnets}

A different approach to create a magnetic trap is the use of microelectrodes, which will act as micro electromagnets when conducting a current. Usually, these microelectrodes are coated with an insulating layer to prevent direct contact between cell and the integrated electrodes and to minimize fluid heating. WirixSpeetjens and de Boeck [80] showed the ability to entrap single magnetic microbeads using a saw-tooth gold microstructure. By switching the direction of the magnetic field, these beads could be transported controllably.

Lee et al. [81] used a gold wire and a matrix of gold microelectrodes to transport and spatially control magnetotactic bacteria (Fig. 2-6B). In a recent report of Mitterboeck et al. [82] single magnetic particles and Jurkat cells, labelled with magnetic beads, were trapped in engineered microtraps by creating local magnetic fields using integrated microelectrodes. 


\subsubsection{Paramagnetic structures}

A third method of magnetic trapping in microfluidic systems depends on integrated paramagnetic structures. By applying an external magnetic field, gradients of high and low magnetic field strength are generated near the paramagnetic structures to entrap particles or cells. A good example is presented in a report of Koschwanez, Carlson and Meldrum [84]. They showed the entrapment of single yeast cells at the tip of a paramagnetic tweezer, which consisted of a Co-Ni-B alloy. Liu et al. [85] reported upon the design of a magnetic single cell microassay. They showed the use of a permalloy layer and an external electrical field to create regions with high magnetic flux densities to entrap immunomagnetically-labelled Jurkat cells.

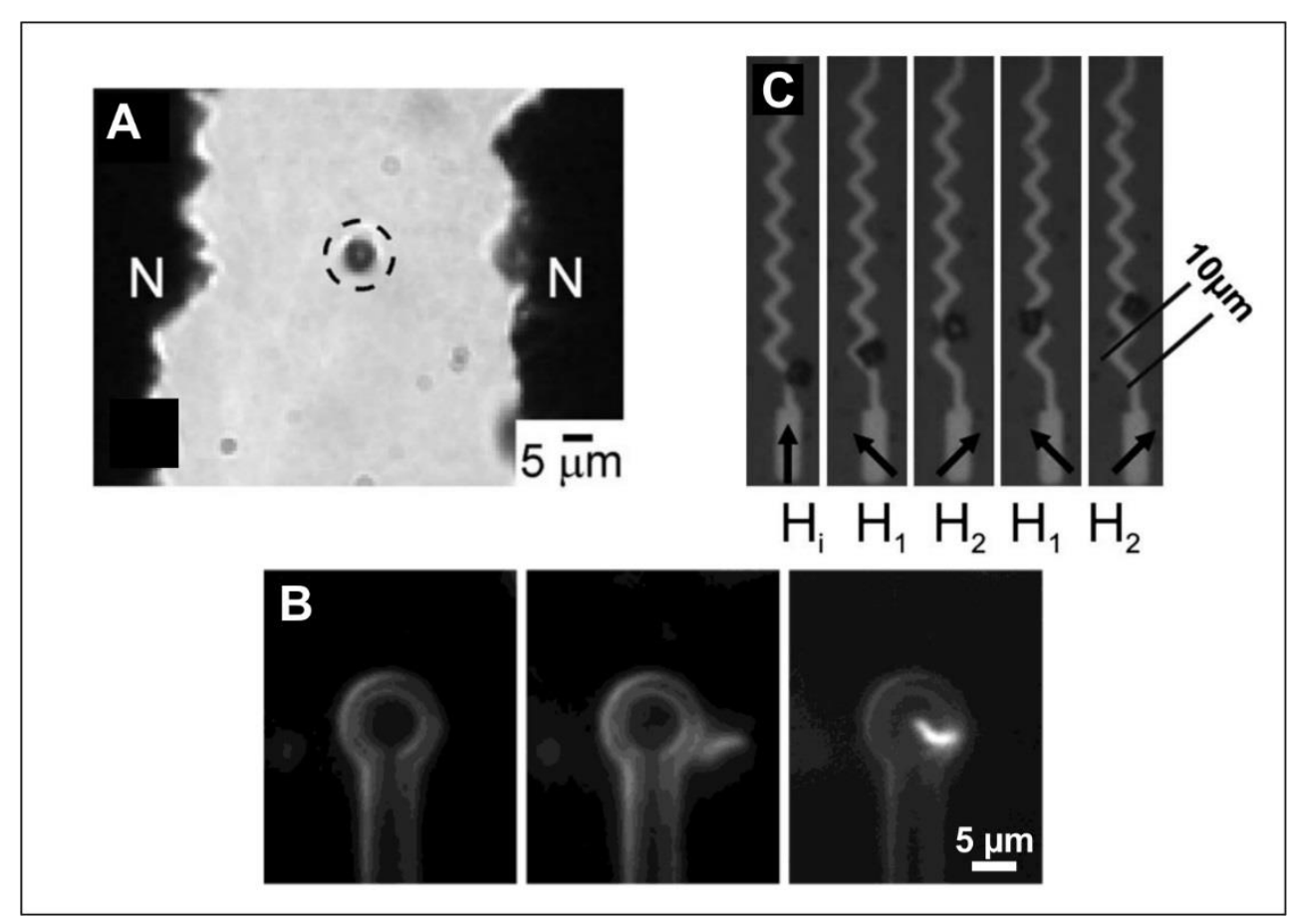

Fig. 2-6: Magnetic trapping of single cells. A) Winkleman et al. [79] used integrated permanent magnets to entrap a single yeast cell, B) Lee et al. [81] used a micro-electromagnet to attract and entrap single magnetotactic bacteria at a high magnetic field gradient. C) Donolato et al. [83] developed a zig-zag permalloy structure, which was used to entrap and transport single yeast cells by changing the direction of the external magnetic field. 
In their device, the amount of cells per trap was dependent on the trap size. Similarly, Donolato et al. [83] used a zig-zag permalloy $\left(\mathrm{Ni}_{80}-\mathrm{Fe}_{20}\right)$ structure to trap and spatially transport single yeast cells, labelled with magnetic beads, by directing an external magnetic field (Fig. 2-6C). Trapping forces were estimated to be $40 \mathrm{pN}$ when using micron-sized permalloy structures [83]. Furthermore, they showed cell survival of trapped yeast cells for over $16 \mathrm{~h}$ and after long term trapping, the cells could be released by switching off the external field. Other reports have shown cell trapping and spatial controllability using paramagnetic alloys shaped as concentric structures [86] and circular disks [87].

An interesting approach of magnetic manipulation of single sperm is reported by Khalil et al. [88]. They used $50 \mu \mathrm{m}$ long microtubes with a diameter of 5-8 $\mu \mathrm{m}$, which consisted of rolled-up Ti/Fe nanomembranes, in combination with bovine sperm cells to create micro-bio-robots. Upon coupling between sperm cell and microtube, weak magnetic fields were used to direct the microtube using its magnetic dipole moment while exploiting the flagellum-induced momentum to move the micro-biorobot towards its desired location.

\subsubsection{Design considerations}

Magnetic fields are known to be non-invasive to biological tissues. When labelled with MNPs, external magnetic fields can be used to create high trapping forces on cells. A drawback of using micro-electromagnets is the generation of high fluid temperatures when applying high currents [89]. Fluid heating is also a known problem when using optical and electrical methods. This problem can be solved by using externally-applied magnetic fields, in which an increase of field strength does not affect the fluid temperature. Furthermore, similarly to optical and electrical methods, cell trapping can be reversed by switching off the applied field. However, a magnetic trap depends on intrinsic magnetic properties of the cells or the extrinsic magnetic properties by immunolabeling MNPs. Most cells do not possess strong intrinsic magnetic properties and therefore, the specificity of a magnetic trap depends upon the specificity of available antibodies for immunolabeling.

\subsection{Acoustic trapping}

A relatively new approach for cell trapping is the use of acoustics to create standing waves in a fluid. These standing waves generate stationary pressure gradients, which will exert a force on an object when its density and/or compressibility are different compared to the surrounding medium. Comparable to DEP, this force 
(acoustical radiation force) will attract or repulse an object. The pressure gradients are called pressure nodes in case of minimum pressure amplitude and pressure antinodes in case of maximum pressure amplitude. Since biological cells usually have a higher density compared to the medium, they will move towards the pressure nodes with lowest pressure amplitude [90, 91].

\subsubsection{Bulk acoustical waves}

Cell manipulation and entrapment can be achieved by using bulk acoustical waves (BAW) in bulk liquid, capillaries [95, 96] or microfluidic systems [97]. These waves can be generated by opposing transducers, single focused transducers with reflectors and layered resonators [90]. BAW have been used to entrap clusters of adherent COS-7 cells [98], HEP-G2 cells [99], neuronal stem cells and yeast cells [97] and erythrocytes [100]. Furthermore, BAW has been used for the entrapment of sperm cells in a microfluidic system for separation from epithelial cell lysate [101]. However, few reports show the potential to manipulate cells on the single cell level since the trapping resolution is limited. An exception is reported by Evander et al. [97]. They reported upon the entrapment of individual plastic beads with a diameter of $10 \mu \mathrm{m}$ and calculated a trapping force of $430 \pm 135 \mathrm{pN}$.

\subsubsection{Surface acoustic waves}

A different approach to generate standing waves is the use of interdigital transducers (IDTs), which are fabricated on the surface of piezoelectric substrates [102]. These IDTs are arranged in parallel or orthogonal orientation [91]. Upon applying an appropriate electrical field, these IDTs generate surface acoustic waves (SAW), which propagate over the surface of the substrate (Fig. 2-7A), creating standing surface acoustic waves (SSAW). These SSAW have been used for cell patterning [91, 92], separation [103] and focusing [104, 105] in microfluidic systems. SSAW have been used by Ding et al. [92] to entrap single plastic beads, bovine erythrocytes and C. Elegans. Furthermore, they showed spatial manipulation of particles and cells at a high resolution (Fig. 2-7B). Moreover, no adverse effects were observed on cell viability and proliferation. In a report of Guo et al. [93] SSAW were used to control the cell-cell interaction by the spatial arrangement of human embryonic kidney cells within the $\mu \mathrm{m}$ resolution (Fig. 2-7C). Using this technique, they showed gap junctional dye transfer between cell pairs of homotypic and heterotypic cells and between adherent or suspended cells. 


\subsubsection{Acoustic tweezers}

Similar to optical trapping, single focused acoustic beams can be used for cell trapping. In both techniques, a single Gaussian beam is used to pull a cell towards the beam axis. Several reports show the use of a single beam for trapping and spatial manipulation (within the $\mu \mathrm{m}$ resolution) using ultrasound in the $20-200 \mathrm{MHz}$ range [94, 106-110]. A good example is reported by Lee et al. [94] in which individual leukaemia cells were spatially manipulated (Fig. 2-7D-E). Exerted forces using an ultrasound beam vary within the 10-400 nN range [111]. The effect of ultrasound beam trapping on cell viability have not been widely explored, although no adverse effects were observed on cell viability by Hwang et al. [106].

\subsubsection{Design considerations}

Cell trapping using ultrasound is a relatively new field, which has several advantages over previously described methods. Three different methods for acoustical trapping are reported: trapping by BAW, SAW or a single ultrasound beam. Devices utilizing BAW are versatile in steering and focusing the acoustical trap, but are more suitable to trap groups of cells rather than single cells [112]. Devices utilizing SAW can be used to trap single cells and are easily integrated in microfluidic systems, however their typical trapping resolution is lower compared to other trapping methods [3]. Ultrasound beams have shown excellent single cell trapping capability with high resolution, utilizing beams with a lower power density compared to optical systems with similar holding forces [3]. As a result, the effects of ultrasound trapping on cell viability are moderate [113]. Furthermore, deeper beam penetration can be established using ultrasound. The throughput of single cell entrapment by acoustic beams is low. Increasing trapping throughput can be established more easily by using SAW type devices.

\subsection{Applicability towards electrical analysis of single sperm}

The previous paragraphs provided an overview upon single cell trapping using hydrodynamic, electrical, chemical, optical, magnetic and acoustic methods towards single cell analysis applications. A limited number of reports have shown the potential to entrap and/or spatially manipulate individual sperm cells. These reports are summarized in Table 2-1. 


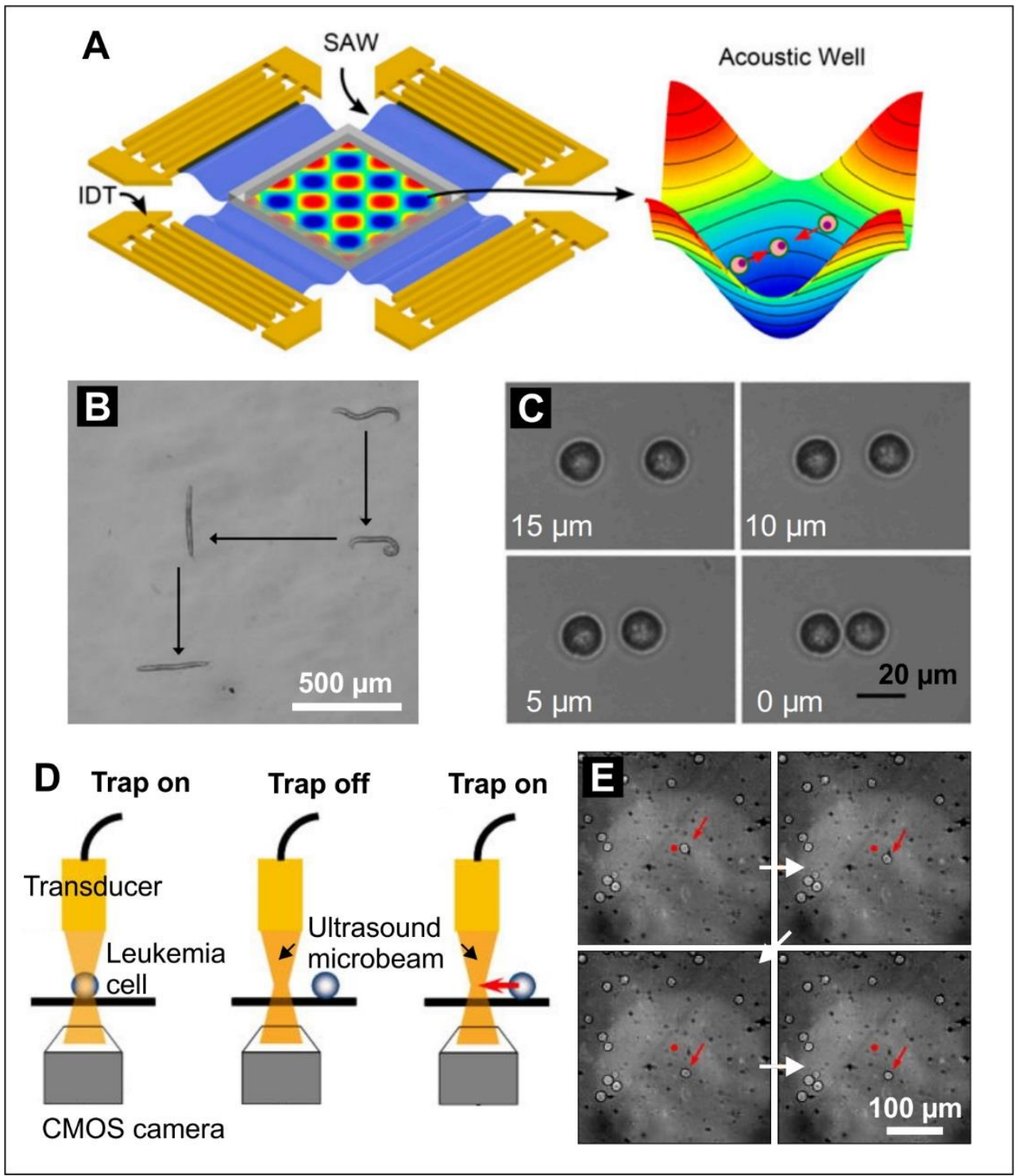

Fig. 2-7: Single cell trapping using acoustics can be accomplished using surface acoustic waves and ultrasonic microbeams. Ding et al. [92] and Guo et al. [93] used A) orthogonal IDTs for the entrapment and spatial manipulation of $B$ ) C.Elegans and C) human embryonic kidney cells. D\&E) Lee et al. [94] used an ultrasound microbeam to spatially manipulate individual leukaemia cells. 
As mentioned in the introduction of this chapter, we aim to analyse individual sperm cells using impedance spectroscopy, requiring spatial confinement of single cells. To select the best trapping strategy for this application, a list of criteria is constructed (Table 2-2). The trapping technique must confine individual sperm cells for a duration of at least 1 min. After trapping and analysis, the sperm cell must be released and should be minimally damaged. The fabrication/system complexity describes the accessibility and the ease of application of the specific trapping method in microfluidic systems. Finally, integration of the trapping method with microelectrodes is essential, which are required for electrical analysis.

Hydrodynamic trapping of sperm, as described in chapter 4, is a straightforward way to entrap individual cells. Stable and non-invasive trapping was achieved by controlling the fluid flows in two separate channels, which could be reversed by reversing the fluid flow. Although this method depends upon the fabrication of thin multilayer structures, requiring state-of-the-art cleanroom facilities, it has good potential to be integrated with microelectrodes. Electrical trapping, as described by Fuhr et al. [44], utilizes microelectrodes to induce nDEP trapping forces on individual sperm cell. Stable and reversible trapping was accomplished, although temporal immobilization of motile sperm cells was observed due to applied electrical fields. Integration of measurement electrodes in a microfluidic system containing trapping electrodes can be easily accomplished, although crosstalk between the electrical trapping and measurement systems might affect measurement precision. Using optical tweezers, as reported by Ohta et al. [75] and Nascimento et al. [74, 76], sperm cells can be confined in high spatial resolution, long duration and in reversible fashion. Although this trapping method can be used to position a cell in between integrated microelectrodes, long trap duration has shown a negative effect on sperm [74]. As an additional disadvantage, optical tweezers are complex and expensive systems. Chemical trapping was performed by Frimat et al. [56] using $10 \mu \mathrm{m}$ wide fibronectin spots, which were fabricated using $\mu \mathrm{CP}$. Although this method is relatively simple and allowed sperm trapping in a stable and non-invasive fashion, single sperm trapping efficiency is low and trapping cannot be easily switched on or off. Furthermore, due to manual fabrication steps in $\mu \mathrm{CP}$, integration and alignment with microelectrodes could be challenging. Finally, magnetic and acoustic methods have been applied on sperm cells, but do not yield the required resolution for single cell confinement. 
Table 2-1: Reports upon trapping of (single) spermatozoa using hydrodynamic, electrical, chemical, optical, magnetic or acoustic methods.

\begin{tabular}{|c|c|c|c|}
\hline & Method & Summary & Remarks / Complications \\
\hline $\begin{array}{l}\text { de Wagenaar } \\
\text { et al. [12] }\end{array}$ & $\begin{array}{l}\text { Hydro- } \\
\text { dynamic }\end{array}$ & $\begin{array}{l}\text { Single boar sperm cells were trapped } \\
\text { hydrodynamically in maximum } 20 \text { side } \\
\text { channels, which had a width of } 2 \mu \mathrm{m} \\
\text { and a height of } 1 \mu \mathrm{m} \text {. }\end{array}$ & $\begin{array}{l}\text { Clogging of the side } \\
\text { channels affected the cell } \\
\text { trapping efficiency. }\end{array}$ \\
\hline $\begin{array}{l}\text { Fuhr et al. } \\
{[44]}\end{array}$ & Electrical & $\begin{array}{l}\text { Single, motile human were entrapped } \\
\text { in octopole DEP traps ( } 8 \mathrm{MHz}, 2.5 \mathrm{~V}) \\
\text { and in interdigitated electrode arrays } \\
(5 \mathrm{MHz}, 2.5 \mathrm{~V}) \text {. }\end{array}$ & $\begin{array}{l}\text { Electrical trapping } \\
\text { resulted in fluid heating }(< \\
\left.5 \quad{ }^{\circ} \mathrm{C}\right) \text { and temporal } \\
\text { immobilization of motile } \\
\text { sperm cells. }\end{array}$ \\
\hline $\begin{array}{l}\text { Frimat et al. } \\
{[56]}\end{array}$ & Chemical & $\begin{array}{l}\text { Single, motile boar sperm cells were } \\
\text { entrapped on } 10 \mu \mathrm{m} \text { wide fibronectin } \\
\text { islands, which were fabricated on a } \\
\text { glass substrate using } \mu \mathrm{CP} \text {. }\end{array}$ & $\begin{array}{l}\text { Large device to device } \\
\text { variation and hard to } \\
\text { integrate with micro } \\
\text { electrodes. }\end{array}$ \\
\hline $\begin{array}{l}\text { Ohta et al. } \\
{[75]}\end{array}$ & Optical & $\begin{array}{l}\text { Optoelectronic tweezers }\left(40 \mathrm{~mW} \cdot \mathrm{cm}^{-2}\right) \\
\text { were used to characterize human } \\
\text { sperm cell viability based on optically } \\
\text { induced DEP, which is affected by the } \\
\text { cell membrane integrity. }\end{array}$ & $\begin{array}{l}\text { The use of low conductive } \\
\text { isotonic buffer }(10 \mathrm{~ms} \cdot \mathrm{m} \\
\left.{ }^{1}\right) \text { may disturb normal cell } \\
\text { physiology. }\end{array}$ \\
\hline $\begin{array}{l}\text { Nascimento } \\
\text { et al. [74] and } \\
{[76]}\end{array}$ & Optical & $\begin{array}{l}\text { Optical tweezers were used to } \\
\text { investigate the swimming force and } \\
\text { velocity of canine and primate sperm } \\
\text { cells. }\end{array}$ & $\begin{array}{l}\text { Trapping canine sperm } \\
\text { cells for } 15 \mathrm{~s} \text { at a laser } \\
\text { power of } 420 \mathrm{~mW} \\
\text { resulted in reduced sperm } \\
\text { motility. }\end{array}$ \\
\hline $\begin{array}{l}\text { Khalil et al. } \\
{[88]}\end{array}$ & Magnetic & $\begin{array}{l}\text { Trapping of individual, motile bovine } \\
\text { sperm in Ti/Fe microtubes, forming } \\
\text { micro-bio-robots, which could be } \\
\text { steered by weak magnetic fields and } \\
\text { flagella propulsion. }\end{array}$ & $\begin{array}{l}\text { Trapping of sperm cells in } \\
\text { the microtubes is non- } \\
\text { specific and cannot be } \\
\text { controlled. }\end{array}$ \\
\hline $\begin{array}{l}\text { Norris et al. } \\
{[101]}\end{array}$ & Acoustic & $\begin{array}{l}\text { BAW were used to entrap and separate } \\
\text { human sperm cells from epithelial cell } \\
\text { lysate for forensic analysis of sexual } \\
\text { assault evidence. }\end{array}$ & $\begin{array}{l}\text { The trapping resolution of } \\
\text { BAW is too small for single } \\
\text { cell trapping. }\end{array}$ \\
\hline
\end{tabular}


As summarized in Table 2-2, hydrodynamic and electrical methods are most suitable for reversible and non-invasive trapping of individual sperm cells for electrical analysis. Although electrical trapping is equally capable of trapping single sperm cells, it could inflict cell damage during long term trapping and could interfere with electrical analysis. Therefore, a hydrodynamic trapping method is preferred to perform electrical analysis on single sperm.

Table 2-2: Trapping criteria for the spatial confinement single sperm cells. The colours indicate, whether the selection criteria are met. Green: yes/high. Orange: moderate. Red: no/low.

\begin{tabular}{|l|c|c|c|c|c|c|}
\cline { 2 - 6 } \multicolumn{1}{c|}{} & $\begin{array}{c}\text { Single cell } \\
\text { potential }\end{array}$ & $\begin{array}{c}\text { Stable } \\
\text { trapping } \\
>1 \mathrm{~min}\end{array}$ & $\begin{array}{c}\text { Reversible } \\
\text { trapping } \\
\text { (on/off) }\end{array}$ & $\begin{array}{c}\text { No cell } \\
\text { damage }\end{array}$ & $\begin{array}{c}\text { Fabrication/ } \\
\text { system } \\
\text { complexity }\end{array}$ & $\begin{array}{c}\text { Device } \\
\text { integration } \\
\text { potential }\end{array}$ \\
\hline $\begin{array}{l}\text { Hydro- } \\
\text { dynamic }\end{array}$ & & & & & & \\
\hline Electrical & & & & & & \\
\hline Optical & & & & & & \\
\hline Chemical & & & & & & \\
\hline Magnetic & & & & & & \\
\hline Acoustic & & & & & & \\
\hline
\end{tabular}

\subsection{Conclusions}

This chapter provided an overview of the most important methods for single cell trapping, which are or can be employed in microfluidic systems. Entrapment of individual cells in a well-defined environment enables cell analysis on the single cell level, which has been exploited for fundamental biological studies and clinical applications. The choice of trapping method depends on factors such as the type of analysis technique, duration of the experiment, required throughput and required spatial controllability. For example, long term and non-invasive trapping of single cells can best be performed by hydrodynamic, magnetic and ultrasound techniques. For high spatial controllability, optical and acoustical beams are most suitable. Obtaining a high number of individually entrapped cells can best be accomplished using hydrodynamic methods. The choice of trapping method is therefore very dependent on the nature of experiment and the analysis method for single cell analysis. For electrical analysis of individual sperm using impedance spectroscopy, requiring integration of a trapping technique with microelectrodes for analysis, hydrodynamic cell entrapment is preferred. 


\subsection{References}

1. Lo, S.J. and D.J. Yao, Get to Understand More from Single-Cells: Current Studies of Microfluidic-Based Techniques for Single-Cell Analysis. Int J Mol Sci, 2015. 16(8): p. 16763-77.

2. Mu, X., et al., Microfluidics for manipulating cells. Small, 2013. 9(1): p. 9-21.

3. Nilsson, J., et al., Review of cell and particle trapping in microfluidic systems. Anal Chim Acta, 2009. 649(2): p. 141-57.

4. Duffy, D.C., et al., Rapid Prototyping of Microfluidic Systems in Poly(dimethylsiloxane). Anal Chem, 1998. 70(23): p. 4974-84.

5. Qin, D., Y.N. Xia, and G.M. Whitesides, Rapid prototyping of complex structures with feature sizes larger than 20 um. Adv. Mater., 1996. 8(11): p. 917-19.

6. McDonald, J.C., et al., Fabrication of microfluidic systems in poly(dimethylsiloxane). Electrophoresis, 2000. 21(1): p. 27-40.

7. Brehm-Stecher, B.F. and E.A. Johnson, Single-cell microbiology: tools, technologies, and applications. Microbiol Mol Biol Rev, 2004. 68(3): p. 53859.

8. Yang, M., C.W. Li, and J. Yang, Cell docking and on-chip monitoring of cellular reactions with a controlled concentration gradient on a microfluidic device. Anal Chem, 2002. 74(16): p. 3991-4001.

9. Valero, A., et al., Apoptotic cell death dynamics of HL6O cells studied using a microfluidic cell trap device. Lab Chip, 2005. 5(1): p. 49-55.

10. Zhu, Z., et al., Real-time monitoring of immobilized single yeast cells through multifrequency electrical impedance spectroscopy. Anal Bioanal Chem, 2014. 406(27): p. 7015-25.

11. Long, Z., et al., Microfluidic chemostat for measuring single cell dynamics in bacteria. Lab Chip, 2013. 13(5): p. 947-54.

12. de Wagenaar, B., et al., Microfluidic single sperm entrapment and analysis. Lab Chip, 2015. 15(5): p. 1294-301.

13. Osada, K., et al., Monitoring of cellular behaviors by microcavity arraybased single-cell patterning. Analyst, 2014. 139(2): p. 425-30.

14. Han, A. and A.B. Frazier, lon channel characterization using single cell impedance spectroscopy. Lab Chip, 2006. 6(11): p. 1412-14.

15. Swennenhuis, J.F., et al., Self-seeding microwell chip for the isolation and characterization of single cells. Lab Chip, 2015. 15(14): p. 3039-46.

16. Yang, Y., et al., Microfluidic device for DNA amplification of single cancer cells isolated from whole blood by self-seeding microwells. Lab Chip, 2015. 15(22): p. 4331-7. 
17. Tan, W.H. and S. Takeuchi, A trap-and-release integrated microfluidic system for dynamic microarray applications. Proc Natl Acad Sci USA, 2007. 104(4): p. 1146-51.

18. Kobel, S., et al., Optimization of microfluidic single cell trapping for longterm on-chip culture. Lab Chip, 2010. 10(7): p. 857-63.

19. Wang, Y., et al., A microfluidic digital single-cell assay for the evaluation of anticancer drugs. Anal Bioanal Chem, 2015. 407(4): p. 1139-48.

20. Sochol, R.D., et al., Hydrodynamic resettability for a microfluidic particulate-based arraying system. Lab Chip, 2012. 12(23): p. 5051-6.

21. Di Carlo, D., N. Aghdam, and L.P. Lee, Single-cell enzyme concentrations, kinetics, and inhibition analysis using high-density hydrodynamic cell isolation arrays. Anal Chem, 2006. 78(14): p. 4925-30.

22. Di Carlo, D., L.Y. Wu, and L.P. Lee, Dynamic single cell culture array. Lab Chip, 2006. 6(11): p. 1445-9.

23. Skelley, A.M., et al., Microfluidic control of cell pairing and fusion. Nat Methods, 2009. 6(2): p. 147-52.

24. Zhang, K., et al., Block-Cell-Printing for live single-cell printing. Proc Natl Acad Sci U S A, 2014. 111(8): p. 2948-53.

25. Kemna, E.W., et al., On chip electrofusion of single human $B$ cells and mouse myeloma cells for efficient hybridoma generation. Electrophoresis, 2011. 32(22): p. 3138-46.

26. Voldman, J., Dielectrophoretic Traps for Cell Manipulation, in BioMEMS and Biomedical Nanotechnology, M. Ferrari, R. Bashir, and S. Wereley, Editors. 2007, Springer US. p. 159-86.

27. Cetin, B. and D. Li, Dielectrophoresis in microfluidics technology. Electrophoresis, 2011. 32(18): p. 2410-27.

28. Khoshmanesh, K., et al., Dielectrophoretic platforms for bio-microfluidic systems. Biosens Bioelectron, 2011. 26(5): p. 1800-14.

29. Qian, C., et al., Dielectrophoresis for bioparticle manipulation. Int J Mol Sci, 2014. 15(10): p. 18281-309.

30. Hughes, M.P. and H. Morgan, Dielectrophoretic trapping of single submicrometre scale bioparticles. J Phys D Appl Phys, 1998. 31(17): p. 2205-10.

31. Morgan, H., M.P. Hughes, and N.G. Green, Separation of submicron bioparticles by dielectrophoresis. Biophys J, 1999. 77(1): p. 516-25.

32. Rosenthal, A. and J. Voldman, Dielectrophoretic traps for single-particle patterning. Biophys J, 2005. 88(3): p. 2193-205.

33. Rosenthal, A., B.M. Taff, and J. Voldman, Quantitative modeling of dielectrophoretic traps. Lab Chip, 2006. 6(4): p. 508-15.

34. Mittal, N., A. Rosenthal, and J. Voldman, nDEP microwells for single-cell patterning in physiological media. Lab Chip, 2007. 7(9): p. 1146-53. 
35. Jang, L.S., P.H. Huang, and K.C. Lan, Single-cell trapping utilizing negative dielectrophoretic quadrupole and microwell electrodes. Biosens Bioelectron, 2009. 24(12): p. 3637-44.

36. Wang, C.C., et al., Adjustable trapping position for single cells using voltage phase-controlled method. Biosens Bioelectron, 2013. 49: p. 297-304.

37. Lan, K.C. and L.S. Jang, Integration of single-cell trapping and impedance measurement utilizing microwell electrodes. Biosens Bioelectron, 2011. 26(5): p. 2025-31.

38. Lombardini, M., et al., Horizontal nDEP cages within open microwell arrays for precise positioning of cells and particles. Lab Chip, 2010. 10(9): p. 12047.

39. Thomas, R.S., H. Morgan, and N.G. Green, Negative DEP traps for single cell immobilisation. Lab Chip, 2009. 9(11): p. 1534-40.

40. Schnelle, T., et al., Three-dimensional electric field traps for manipulation of cells--calculation and experimental verification. Biochim Biophys Acta, 1993. 1157(2): p. 127-40.

41. Voldman, J., et al., Design and analysis of extruded quadrupolar dielectrophoretic traps. J Electrostat, 2003. 57(1): p. 69-90.

42. Bocchi, M., et al., Dielectrophoretic trapping in microwells for manipulation of single cells and small aggregates of particles. Biosens Bioelectron, 2009. 24(5): p. 1177-83.

43. Schnelle, T., T. Muller, and G. Fuhr, Trapping in AC octode field cages. J Electrostat, 2000. 50(1): p. 17-29.

44. Fuhr, G., et al., High-frequency electric field trapping of individual human spermatozoa. Hum Reprod, 1998. 13(1): p. 136-41.

45. Voldman, J., et al., A microfabrication-based dynamic array cytometer. Anal Chem, 2002. 74(16): p. 3984-90.

46. Suehiro, J. and R. Pethig, The dielectrophoretic movement and positioning of a biological cell using a three-dimensional grid electrode system. J Phys D Appl Phys, 1998. 31(22): p. 3298-05.

47. Qian, L., et al., Integrated planar concentric ring dielectrophoretic (DEP) levitator. J Electrostat, 2002. 55(1): p. 65-79.

48. Gray, D.S., et al., Dielectrophoretic registration of living cells to a microelectrode array. Biosens Bioelectron, 2004. 19(12): p. 1765-74.

49. Taff, B.M. and J. Voldman, A scalable addressable positive-dielectrophoretic cell-sorting array. Anal Chem, 2005. 77(24): p. 7976-83.

50. Bhattacharya, S., T.C. Chao, and A. Ros, Insulator-based dielectrophoretic single particle and single cancer cell trapping. Electrophoresis, 2011. 32(18): p. 2550-8. 
51. Bhattacharya, S., et al., Selective trapping of single mammalian breast cancer cells by insulator-based dielectrophoresis. Anal Bioanal Chem, 2014. 406(7): p. 1855-65.

52. Cetin, B. and D.Q. Li, Dielectrophoresis in microfluidics technology. Electrophoresis, 2011. 32(18): p. 2410-27.

53. Falconnet, D., et al., Surface engineering approaches to micropattern surfaces for cell-based assays. Biomaterials, 2006. 27(16): p. 3044-63.

54. Kane, R.S., et al., Patterning proteins and cells using soft lithography. Biomaterials, 1999. 20(23-24): p. 2363-76.

55. $\mathrm{Xu}, \mathrm{L}$., et al., Microcontact printing of living bacteria arrays with cellular resolution. Nano Lett, 2007. 7(7): p. 2068-72.

56. Frimat, J.P., et al., Make it spin: individual trapping of sperm for analysis and recovery using micro-contact printing. Lab Chip, 2014. 14(15): p. 263541.

57. Chen, C.S., et al., Micropatterned surfaces for control of cell shape, position, and function. Biotechnol Prog, 1998. 14(3): p. 356-63.

58. Dike, L.E., et al., Geometric control of switching between growth, apoptosis, and differentiation during angiogenesis using micropatterned substrates. In Vitro Cell Dev Biol Anim, 1999. 35(8): p. 441-8.

59. Wang, N., et al., Micropatterning tractional forces in living cells. Cell Motil Cytoskeleton, 2002. 52(2): p. 97-106.

60. Kilian, K.A., et al., Geometric cues for directing the differentiation of mesenchymal stem cells. Proc Natl Acad Sci USA, 2010. 107(11): p. 4872-7.

61. McBeath, R., et al., Cell shape, cytoskeletal tension, and RhoA regulate stem cell lineage commitment. Dev Cell, 2004. 6(4): p. 483-95.

62. Weibel, D.B., et al., Bacterial printing press that regenerates its ink: Contact-printing bacteria using hydrogel stamps. Langmuir, 2005. 21(14): p. 6436-42.

63. Zhang, H. and K.K. Liu, Optical tweezers for single cells. J R Soc Interface, 2008. 5(24): p. 671-90.

64. Ramser, K. and D. Hanstorp, Optical manipulation for single-cell studies. J Biophotonics, 2010. 3(4): p. 187-206.

65. Jonas, A. and P. Zemanek, Light at work: the use of optical forces for particle manipulation, sorting, and analysis. Electrophoresis, 2008. 29(24): p. 481351.

66. Lim, C.T., et al., Large deformation of living cells using laser traps. Acta Mater, 2004. 52(7): p. 1837-45.

67. Eriksson, E., et al., A microfluidic device for reversible environmental changes around single cells using optical tweezers for cell selection and positioning. Lab Chip, 2010. 10(5): p. 617-25. 
68. Eriksson, E., et al., Optical manipulation and microfluidics for studies of single cell dynamics. J Opt A Pure Appl Op, 2007. 9(8): p. S113-21.

69. Monat, C., et al., Optofluidics: a novel generation of reconfigurable and adaptive compact architectures. Microfluid Nanofluid, 2008. 4(1-2): p. 8195.

70. Lawton, P.F., C.D. Saunter, and J.M. Girkin, Combining optical trapping in a microfluidic channel with simultaneous micro-Raman spectroscopy and motion detection. Microfluidics, Biomems, and Medical Microsystems XII, 2014. 8976.

71. Ramser, K., et al., The combination of resonance Raman spectroscopy, optical tweezers and microfluidic systems applied to the study of various heme-containing single cells. Spectrosc-Int J, 2008. 22(4): p. 287-95.

72. Liberale, $\mathrm{C}$. , et al., Integrated microfluidic device for single-cell trapping and spectroscopy. Sci Rep, 2013. 3: p. 1-6.

73. Liberale, C., et al. Single-cell fluorescence spectroscopy and trapping by microtweezers integrated in a microfluidic circuit. in Frontiers in Optics 2012/Laser Science XXVIII. 2012. Rochester, New York: Optical Society of America.

74. Nascimento, J.M., et al., Analysis of sperm motility using optical tweezers. J Biomed Opt, 2006. 11(4): p. 044001.

75. Ohta, A.T., et al., Motile and non-motile sperm diagnostic manipulation using optoelectronic tweezers. Lab Chip, 2010. 10(23): p. 3213-7.

76. Nascimento, J.M., et al., The use of optical tweezers to study sperm competition and motility in primates. J R Soc Interface, 2008. 5(20): p. 297302.

77. Han, K.H. and A.B. Frazier, Paramagnetic capture mode magnetophoretic microseparator for high efficiency blood cell separations. Lab Chip, 2006. 6(2): p. 265-73.

78. Kang, J.H., et al., A combined micromagnetic-microfluidic device for rapid capture and culture of rare circulating tumor cells. Lab Chip, 2012. 12(12): p. 2175-81.

79. Winkleman, A., et al., A magnetic trap for living cells suspended in a paramagnetic buffer. Appl Phys Lett, 2004. 85(12): p. 2411-3.

80. Wirix-Speetjens, R. and J. de Boeck, On-chip magnetic particle transport by alternating magnetic field gradients. IEEE T Magn, 2004. 40(4): p. 1944-6.

81. Lee, H., et al., Controlled assembly of magnetic nanoparticles from magnetotactic bacteria using microelectromagnets arrays. Nano Lett, 2004. 4(5): p. 995-8.

82. Mitterboeck, R., et al. Magnetic microfluidic system for isolation of single cells. in Bio-MEMS and Medical Microdevices II. 2015. Barcelona, Spain. 
83. Donolato, M., et al., Magnetic domain wall conduits for single cell applications. Lab Chip, 2011. 11(17): p. 2976-83.

84. Koschwanez, J.H., R.H. Carlson, and D.R. Meldrum, Easily fabricated magnetic traps for single-cell applications. Rev Sci Instrum, 2007. 78(4): p. 044301.

85. Liu, W., et al., A novel permalloy based magnetic single cell micro array. Lab Chip, 2009. 9(16): p. 2381-90.

86. Huang, C.Y. and Z.H. Wei, Concentric Magnetic Structures for Magnetophoretic Bead Collection, Cell Trapping and Analysis of Cell Morphological Changes Caused by Local Magnetic Forces. PLoS One, 2015. 10(8): p. e0135299.

87. Henighan, T., et al., Manipulation of magnetically labeled and unlabeled cells with mobile magnetic traps. Biophys J, 2010. 98(3): p. 412-7.

88. Khalil, I.S.M., et al., Biocompatible, accurate, and fully autonomous: a sperm-driven micro-bio-robot. Journal of Micro-Bio Robotics, 2014. 9(3-4): p. 79-86.

89. Pamme, N., Magnetism and microfluidics. Lab Chip, 2006. 6(1): p. 24-38.

90. Evander, M. and J. Nilsson, Acoustofluidics 20: applications in acoustic trapping. Lab Chip, 2012. 12(22): p. 4667-76.

91. Shi, J., et al., Acoustic tweezers: patterning cells and microparticles using standing surface acoustic waves (SSAW). Lab Chip, 2009. 9(20): p. 2890-5.

92. Ding, $\mathrm{X}$., et al., On-chip manipulation of single microparticles, cells, and organisms using surface acoustic waves. Proc Natl Acad Sci USA, 2012. 109(28): p. 11105-9.

93. Guo, F., et al., Controlling cell-cell interactions using surface acoustic waves. Proc Natl Acad Sci U S A, 2015. 112(1): p. 43-8.

94. Lee, J., et al., Targeted cell immobilization by ultrasound microbeam. Biotechnol Bioeng, 2011. 108(7): p. 1643-50.

95. Wiklund, M., S. Nilsson, and H.M. Hertz, Ultrasonic trapping in capillaries for trace-amount biomedical analysis. J Appl Phys, 2001. 90(1): p. 421-26.

96. Wiklund, M., et al., Ultrasonic-trap-enhanced selectivity in capillary electrophoresis. Ultrasonics, 2003. 41(4): p. 329-33.

97. Evander, M., et al., Noninvasive acoustic cell trapping in a microfluidic perfusion system for online bioassays. Anal Chem, 2007. 79(7): p. 2984-91.

98. Hultstrom, J., et al., Proliferation and viability of adherent cells manipulated by standing-wave ultrasound in a microfluidic chip. Ultrasound Med Biol, 2007. 33(1): p. 145-51.

99. Liu, J., et al., Functional three-dimensional HepG2 aggregate cultures generated from an ultrasound trap: comparison with HepG2 spheroids. J Cell Biochem, 2007. 102(5): p. 1180-9. 
100. Evander, M., et al. Study of ATP-release from acoustically levitated eryhrocytes. in Micro Total Analysis Systems. 2007. Paris: Chemical and Biological Microsystems Society.

101. Norris, J.V., et al., Acoustic Differential Extraction for Forensic Analysis of Sexual Assault Evidence. Anal Chem, 2009. 81(15): p. 6089-95.

102. Yeo, L.Y. and J.R. Friend, Surface Acoustic Wave Microfluidics. Annual Review of Fluid Mechanics, Vol 46, 2014. 46: p. 379-406.

103. Shi, J., et al., Continuous particle separation in a microfluidic channel via standing surface acoustic waves (SSAW). Lab Chip, 2009. 9(23): p. 3354-9.

104. Shi, J., et al., Three-dimensional continuous particle focusing in a microfluidic channel via standing surface acoustic waves (SSAW). Lab Chip, 2011. 11(14): p. 2319-24.

105. Chen, Y.C., et al., Standing surface acoustic wave (SSAW)-based microfluidic cytometer. Lab Chip, 2014. 14(5): p. 916-23.

106. Hwang, J.Y., et al., Cell membrane deformation induced by a fibronectincoated polystyrene microbead in a 200-MHz acoustic trap. IEEE Trans Ultrason Ferroelectr Freq Control, 2014. 61(3): p. 399-406.

107. Lam, K.H., et al., Ultrahigh frequency lensless ultrasonic transducers for acoustic tweezers application. Biotechnol Bioeng, 2013. 110(3): p. 881-6.

108. Jeong, J.S., et al., Particle manipulation in a microfluidic channel using acoustic trap. Biomed Microdevices, 2011. 13(4): p. 779-88.

109. Yoon, C., et al., Multi-particle trapping and manipulation by a highfrequency array transducer. Appl Phys Lett, 2014. 105(214103): p. 1-4.

110. Lee, J., et al., Single beam acoustic trapping. Appl Phys Lett, 2009. 95(73701): p. 1-3.

111. Baresch, D.T., J.-L. and R. Marchiano Observation of a single-beam gradient force acoustical trap for elastic particles: acoustical tweezers. 2014.

112. Marx, V., Biophysics: using sound to move cells. Nat Methods, 2015. 12(1): p. 41-4.

113. Mulvana, H., S. Cochran, and M. Hill, Ultrasound assisted particle and cell manipulation on-chip. Adv Drug Deliv Rev, 2013. 65(11-12): p. 1600-10. 


\section{Electrical characterization of single cells in microfluidic systems}

Microfluidic technology has been extensively exploited to electrically characterize single cells. After the first application of the Coulter counting principle in a microsystem, numerous studies have focused on the analysis of the dielectric properties of a large variety of cell types, including blood cells, cancer cells, bacteria and yeast. The dielectric properties of a cell describes its potential to conduct current as well as to store current. Various parts of the cell, including the cell membrane and cytoplasm, react differently to imposed electrical fields. These differences can be studied by micro impedance flow cytometry and micro electrical impedance spectroscopy. 


\subsection{Introduction}

Electrical characterization of single cells was first reported by Wallace H. Coulter [1] in the late 1950s. By flowing suspended red blood cells through a small orifice between two containers, the direct current (DC) electrical response of the system was altered. This electrical response, i.e. a drop in transient current (increase of resistance), which was measured using two electrodes at either side of the orifice, was proportional to the cell volume. This information could be used for cell counting and size measurement. This technique, which has found application in haematology particularly, is called Coulter counting. Hoffman et al. [2, 3] used the Coulter principle in the late 1970s to measure changes in impedance using alternating current (AC), allowing cell analysis at higher frequency. In the late 1990s, Larsen et al. [4] fabricated the first micro Coulter counter, in which an aperture-containing microfluidic channel was etched in silicon and microelectrodes were integrated at either side of the aperture for electrical measurements. In following years, advances in photolithography and chip fabrication allowed improvement of the micro Coulter counter, yielding numerous reports on the use of Coulter-type devices for single cell analysis.

Electrical analysis of single cells in microfluidic systems can be roughly divided in three areas: microfluidic impedance cytometry, microfluidic electrical impedance spectroscopy and electrorotation. This chapter provides an overview of the theoretical aspects of single cell spectroscopy and focuses on the application of microfluidic impedance cytometry and impedance spectroscopy. Using microfluidic impedance cytometry, cells are flown through a microfluidic channel containing at least a single electrode pair, which is used to record changes in impedance upon cell passage at a single or multiple discrete frequencies. This method provides information upon cell characteristics such as cell volume, count, morphology, membrane capacitance and cytoplasm conductivity. Microfluidic impedance spectroscopy focuses on the analysis of the dielectrical properties of medium and suspended cells as a function of field frequency. This method allows the investigation of the electrical properties of a single cell over time.

\subsection{Dielectric spectroscopy}

Dielectric spectroscopy, which is also known as (electrochemical) impedance spectroscopy, is an analysis tool which is used to investigate the dielectric properties of materials, tissues and cells as a function of frequency. These dielectric properties are determined by a material's conductivity $\sigma$ and permittivity $\varepsilon$, which 
describe the conduction and storage of current, respectively, in response to an electrical field. These properties are expressed as a complex permittivity $\varepsilon^{*}[5,6]$ :

$$
\varepsilon^{*}=\varepsilon^{\prime}-j \varepsilon^{\prime \prime}
$$

The real part of the permittivity $\varepsilon^{\prime}$ is a measure for the amount of energy stored in the dielectric material. The imaginary part of the permittivity $\varepsilon^{\prime \prime}$, which is also called the loss factor, describes the dissipative and 'lossy' behaviour of a material. The imaginary part of the permittivity $\varepsilon^{\prime \prime}$ is given by [5]:

$$
\varepsilon^{\prime \prime}=\frac{\sigma}{\omega \varepsilon_{0}}
$$

in which $\sigma$ is the conductivity $\left(\mathrm{S} \cdot \mathrm{m}^{-1}\right), \varepsilon_{0}$ is the permittivity of free space or vacuum $\left(8.85 \cdot 10^{-12} \mathrm{~F} \cdot \mathrm{m}^{-1}\right)$ and $\omega$ is the angular frequency (radian $\left.\cdot \mathrm{s}^{-1}\right)$, which is equal to $2 \pi f$ in which $f$ is the frequency $(\mathrm{Hz})$ of the applied AC field. The permittivity of a material is often described by its relative permittivity, i.e. dielectric constant. This property describes the ratio of the complex permittivity of the dielectric material compared to the vacuum permittivity $\varepsilon_{0}$ :

$$
\varepsilon_{r}^{*}=\frac{\varepsilon^{*}}{\varepsilon_{0}}
$$

Although the value of the relative permittivity is complex and consists of a real and imaginary component, in practice, the permittivity of a material is often described by the static relative permittivity $\varepsilon_{r}$, which is the relative permittivity at a frequency of zero. For example, aqueous solutions at $20^{\circ} \mathrm{C}$ have a static relative permittivity of approximately 80 .

\subsubsection{Dielectric dispersion}

Under the influence of external electric fields, tissues and cells are polarized and hereby obtain a macroscopic dipole moment. This polarization is caused by the displacement of charge (e.g. movement of ions) in the polarized object due to the applied electric field. This polarization is not an instantaneous process and before reaching an equilibrium state, a certain period of time elapses. Upon removal of the electric field, the polarization decays, i.e. relaxes to a steady state. When exposing the dielectric object to an AC electric field, its relaxation and correspondingly, its permittivity, will depend on field frequency [5-7]. This dependence is described by dielectric dispersion. 
In biological tissue, roughly three relaxation mechanism are responsible for its dielectric response. Firstly, the $\alpha$-dispersion is caused by diffusion processes of ionic species due to the polarization of the double layer around the object $[5,7,8]$. This dispersion occurs at frequencies within the $\mathrm{kHz}$ range and is influenced by factors such as the membrane potential and counter-ion displacement around charged membranes. Secondly, the $\beta$-dispersion is caused by charging of the capacitive cell membranes, i.e. the $\beta$-dispersion describes the interfacial polarization across the cellular membranes with respect to the extra-cellular electrolyte and intra-cellular cytoplasm $[5,7,8]$. This dispersion occurs within the $\mathrm{MHz}$ regime and the relaxation can be described using the Maxwell-Wagner theory (paragraph 3.3.2). Thirdly, the $\gamma$-dispersion is caused by the dipolar relaxation of water and small molecules within the dielectric object and occurs within the $\mathrm{GHz}$ regime $[5,7,8]$. Small subdispersions have been reported between $\beta$ and $\gamma$-dispersions due to relaxation of large molecules, such as biopolymers, proteins, cellular organelles and proteinbound water [8].

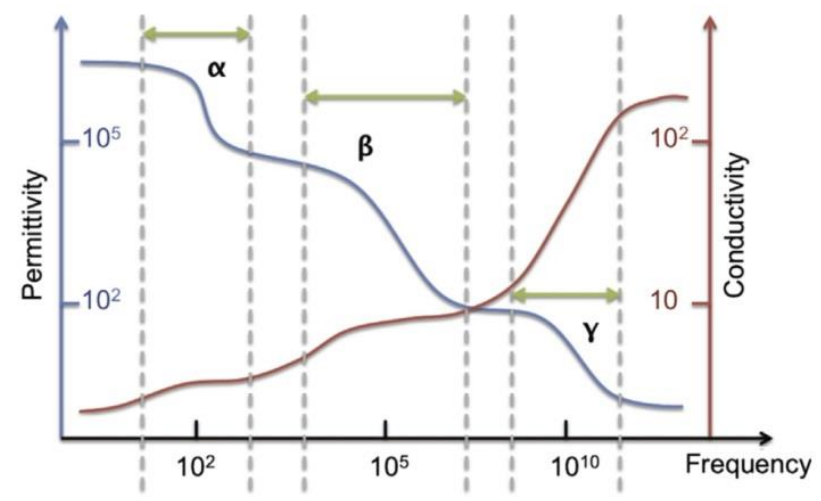

Fig. 3-1: Dielectric dispersion of the real part of the permittivity of biological tissue. Three major dispersion regions are distinguished. Due to these dispersions, the permittivity is decreasing and correspondingly, the conductivity is increasing with increasing field frequency (adapted from [8]).

The effects of dielectric dispersion on the real part of the permittivity are shown in Fig. 3-1. Due to this dispersion, the real part of the permittivity decreases with increasing frequency. Correspondingly, the conductivity increases. Cell analysis using dielectric spectroscopy in microfluidic systems especially focuses on measurements within the $\beta$-dispersion. At frequencies in the $\mathrm{kHz}$ range, the electrical double layer, formed at the electrode-electrolyte interface of the 
microelectrodes (see paragraph 3.3.1), prevents analysis of the $\alpha$-dispersion. At frequencies in between the high $\mathrm{kHz}$ range and low $\mathrm{MHz}$ range, the cell membrane mainly exhibits insulating behaviour. When increasing the frequency to the multiple $\mathrm{MHz}$ range, the permittivity of the membrane interface decreases, allowing ions to flow through the membrane into the cell, allowing investigation of the cell's interior. In the high $\mathrm{MHz}$ range, the parasitic effects of electrode geometry start to dominate.

\subsection{Equivalent circuit modelling}

A straightforward way to gain insight in the electrical properties of a complex system is to construct an equivalent circuit model (ECM). Most parts of the microfluidic system can be modelled as linear components (resistors, capacitors or inductors), which can be used to model the electrical response of the setup. Equivalent models of the microfluidic chip and biological cell are addressed separately.

\subsubsection{Microfluidic chip}

Consider a microfluidic chip that is fabricated of an insulating material (e.g. plastic or glass), which contains a microfluidic channel with two integrated microelectrodes. Several components can be distinguished in this system, which contribute to its electrical response: the electrical leads, the microelectrodes and the electrolyte. Before deriving an expression for the frequency response of a typical microfluidic setup, the electrical response of each component is addressed.

\section{Electrical leads}

The electrical leads on and off-chip are mainly resistive, from which the resistance $R_{\text {lead }}(\Omega)$ can easily calculated by:

$$
R_{\text {lead }}=\frac{\rho L}{A}
$$

in which $\rho$ is the specific resistivity of the lead material $(\Omega \cdot \mathrm{m}), L$ the length of the electrical lead $(\mathrm{m})$, and $A$ the cross-sectional area of the lead $\left(\mathrm{m}^{2}\right)$.

\section{Electrical double layer}

The interface between the microelectrodes and conductive electrolyte plays a major role in the electrical response of the microfluidic setup. In theory, this electrode-electrolyte interface can be modelled by a resistor and capacitor in parallel. This resistor models the charge transfer at the interface by faradaic 
processes, whereas the capacitor is a model for the non-faradaic processes including adsorption and desorption [9]. In the latter case, no charge transfer is taking place, although external current can flow when altering the potential, electrode area or electrolyte composition [9]. When no charge transfer is taking place, regardless of the externally applied potential, the electrode is considered as an ideal polarizable electrode (IPE). In practice, no IPE electrodes exists, although IPE behaviour can be observed over limited potential ranges. In that scenario, no charge crosses the IPE interface when applying low potentials, due to which the electrode-electrolyte interface is purely capacitive [9].

The capacitive behaviour of the electrode-electrolyte interface is influenced by the charged species in the solution. Helmholtz was the first to observe co-ion repulsion and counterion attraction to the surface of charged electrodes when submerged in a liquid ionic conductor (i.e. electrolyte) [9]. As a result, two layers of opposite polarity are present between the electrode and electrolyte, which are called the electrical double layer. Gouy and Chapman improved the Helmholtz model by adding a diffuse layer [9]. As a result, the effects of applied potential and the ionic concentrations could be accounted for. Stern modified this model by combining a fixed layer of adhered ions at the electrode surface, suggested by Helmholtz, with a diffuse layer of ions, proposed by Gouy-Chapman. Eventually, in the GouyChapman-Stern (GCS) model, three different regions are defined: the inner Helmholtz plane defined by a layer of solvent ions, the outer Helmholtz plane defined by a layer of solvated ions and the diffuse layer. [9] The resulting capacitance of the electrical double layer $C_{d}\left(\mathrm{~F} \cdot \mathrm{m}^{-2}\right)$ is defined as [9]:

$$
\frac{1}{C_{d}}=\frac{1}{C_{H}}+\frac{1}{C_{D}}
$$

in which $C_{H}\left(\mathrm{~F} \cdot \mathrm{m}^{-2}\right)$ is the capacitance of the Helmholtz layer and $C_{D}\left(\mathrm{~F} \cdot \mathrm{m}^{-2}\right)$ is the capacitance of the Gouy-Chapman diffuse layer. $C_{H}$ and $C_{D}$ are calculated by [9]:

and

$$
C_{H}=\frac{\varepsilon_{e l} \varepsilon_{0}}{\chi_{O H Z}}
$$

$$
C_{D}=\sqrt{\left(\frac{2 z^{2} e^{2} \varepsilon_{e l} \varepsilon_{0} n^{0}}{k T}\right)} \cosh \left(\frac{z e \varphi_{O H Z}}{2 k T}\right)
$$

with $\varepsilon_{e l}$ the electrolyte permittivity, $\varepsilon_{0}$ the vacuum permittivity $\left(8.85 \cdot 10^{-12} \mathrm{~F} \cdot \mathrm{m}^{-1}\right)$, 
$\chi_{\mathrm{OHZ}}$ the position of the outer Helmholtz layer (positioned at the radius of the adhered, solvated ions) with respect to the electrode surface, $z$ the charge on the ion, e the charge of the electron $\left(1.60 \cdot 10^{-19} \mathrm{C}\right), n^{0}$ the number concentration of each ion in the bulk solution, $\mathrm{k}$ the Boltzmann constant $\left(1.38 \cdot 10^{-23} \mathrm{~J} \cdot \mathrm{K}^{-1}\right), T$ the absolute temperature $(\mathrm{K})$ and $\varphi_{\mathrm{OHZ}}$ the potential $(\mathrm{V})$ at $\chi_{\mathrm{OHZ}}$ with respect to the bulk solution. The double layer $C_{d}$ is described as a capacitance per unit area $\left(\mathrm{F} \cdot \mathrm{m}^{-2}\right)$. The double layer capacitance $C_{D L}(\mathrm{~F})$ is determined by:

$$
C_{D L}=A \cdot C_{d}
$$

in which the $A$ is the surface area of the electrodes $\left(\mathrm{m}^{2}\right)$, which is calculated by multiplying the electrode width $l(\mathrm{~m})$ by the channel width $w(\mathrm{~m})$. When assuming a high conductive environment $\left(C_{H}{ }^{-1}>>C_{D}^{-1}\right)$, the double layer is mainly determined by $C_{H}$ [10] which has a value of approximately $10-40 \mu \mathrm{F} \cdot \mathrm{cm}^{-2}$ [11], depending on the electrode material. In very dilute solutions $\left(C_{H}{ }^{-1}<<C_{D}{ }^{-1}\right), C_{d} \approx C_{D}$.

\section{Electrolyte and measurement cell}

The electrolyte forms an additional barrier for the charge transfer in between the microelectrodes. The electrolyte is mainly conductive and can be modelled by a simple resistor. However, at high frequencies it acts as a dielectric in between the microelectrodes, resulting in a parasitic effect. The resistance of the electrolyte $R_{e l}$ $(\Omega)$ is calculated by $[12,13]$ :

$$
R_{e l}=K \cdot \rho_{e l}
$$

in which $K$ is the cell constant $\left(\mathrm{m}^{-1}\right)$ and $\rho_{e l}$ the electrolyte resistivity $(\Omega \cdot \mathrm{m})$, which is the inverse of the electrolyte conductivity $\sigma_{e l}\left(\Omega^{-1} \cdot \mathrm{m}^{-1}\right.$ or $\left.\mathrm{S} \cdot \mathrm{m}^{-1}\right)$ :

$$
\rho_{e l}=\frac{1}{\sigma_{e l}}
$$

The cell constant is determined by the electrode geometry. In case of two paralleloriented electrodes, the cell constant is given by [13]:

$$
K_{\text {parallel }}=\alpha \cdot \frac{S}{A}
$$

in which $s$ is the electrode spacing (i.e. channel height) (m), $A$ the electrode surface area $\left(\mathrm{m}^{2}\right)$ and $\alpha$ the correction factor for field fringing at the electrode edges. For alternative electrode geometries, such as interdigitated planar electrodes, the cell 
constant is derived differently [14]. For simulations reported in this thesis, no field fringing was assumed $(\alpha=1)$.

\section{Parasitic effects}

Finally, parasitic coupling between the electrodes occurs at high frequencies. In practice, the parasitic capacitance $C_{\text {par }}(\mathrm{F})$ is mainly determined by the electrolyte capacitance $C_{e l}(\mathrm{~F})$, at which $C_{p a r} \approx C_{e l}$. The electrolyte capacitance is given by [12, 14]:

$$
C_{e l}=\frac{\varepsilon_{e l} \varepsilon_{0}}{K}
$$

\section{ECM}

The resulting equivalent model $[15,16]$ (Fig. 3-2A) consists of the lead resistance $\left(R_{\text {lead }}\right)$, double layer capacitance $\left(C_{D L}\right)$, electrolyte resistance $\left(R_{e l}\right)$ and capacitance $\left(C_{e l}\right)$. The equivalent impedance $Z_{E C M}(\Omega)$ of this circuit is derived by:

$$
Z_{E C M}=2 R_{\text {lead }}+X+\frac{2}{j \omega C_{D L}}
$$

in which $X$ is the equivalent impedance of $R_{e l}$ and $C_{e l}$ :

$$
X=\frac{R_{e l}}{1+j \omega R_{e l} C_{e l}}
$$

A typical impedance response is shown in figure Fig. 3-2B. The effects of $C_{D L}$ and $C_{e l}$ are clearly visible as a decrease of the impedance with increasing frequency in the low frequency and high frequency range, respectively. The optimal measurement range can be found within the resistive plateau region, in which the impedance is roughly equal to $R_{e l}$, i.e. when the phase angle is close to $0^{\circ}$. 

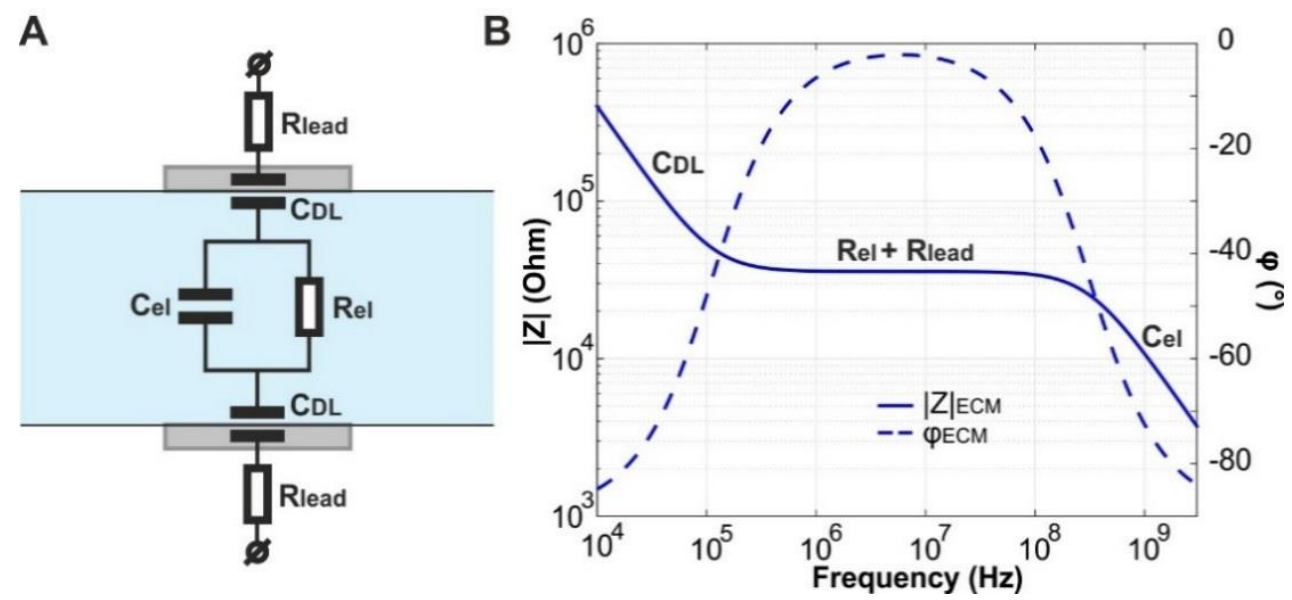

Fig. 3-2: A) ECM of a microfluidic chip with parallel microelectrodes. B) The frequency response of the ECM of a microfluidic setup. At low frequencies, $C_{D L}$ is dominant, whereas at intermediate frequencies ( $\mathrm{MHz}$ range) the impedance is predominantly determined by $R_{e l}$. At high frequencies, capacitance coupling between the electrodes causes the impedance to drop. The following simulation parameters were used: $w$ $=s=l=20 \cdot 10^{-6} \mathrm{~m}, \mathrm{~A}=4 \cdot 10^{-12} \mathrm{~m}^{2}, \sigma_{e l}=1.4 \mathrm{~S} \cdot \mathrm{m}^{-1}, \varepsilon_{e l}=80, R_{\text {lead }}=0 \Omega$, $C_{d}=20 \mu \mathrm{F} \cdot \mathrm{cm}^{-2}, C_{D L}=8 \cdot 10^{-8} \mathrm{~F}, R_{e l}=3.6 \cdot 10^{4} \Omega$ and $C_{e l}=1.42 \cdot 10^{-14} \mathrm{~F}$.

\subsubsection{Single cells in suspension}

A biological cell is a complex system consisting of a large diversity of cell contents, including DNA, mRNA, proteins, cell organelles and membrane structures, each having its own dielectric properties. As described by Pauly and Schwann [17] and later by Foster and Schwann [5], a biological cell can be simplified as a single shelled spheroid, in which the conducting cell interior is surrounded by a non-conducting membrane, which separates the interior from the (conducting) exterior electrolyte. The dielectric properties of a mixture of cells and surrounding medium, as described by the Maxwell mixture equation, which was later extended by Wagner, can be used to calculate the impedance of the total mixture. A different approach to investigate the impedance is by constructing an ECM.

\section{Maxwell-mixture theory}

Maxwell was the first to describe the conductivity of a solution containing colloidal particles. His theory, called the Maxwell's mixture theory, relates the conductive properties of the particle to the conductive properties of the mixture [18]: 


$$
\frac{\sigma_{m i x}-\sigma_{e l}}{\sigma_{m i x}+2 \sigma_{e l}}=\varphi \frac{\sigma_{p}-\sigma_{e l}}{\sigma_{p}+2 \sigma_{e l}}
$$

in which $\sigma_{\text {mix }}$ and $\sigma_{p}$ are the conductivities $\left(\mathrm{S} \cdot \mathrm{m}^{-1}\right)$ of the mixture and the particle, respectively and $\varphi$ is the volume fraction. This theory was extended by Wagner to the case of AC excitation at low volume fractions $(<10 \%)$. The complex permittivity of a mixture $\varepsilon_{m i x}^{*}$ is given by $[15,19]$ :

$$
\varepsilon_{m i x}^{*}=\frac{1+2 \varphi f_{C M}^{*}}{1-\varphi f_{C M}^{*}} \text { with } f_{C M}^{*}=\frac{\varepsilon_{p}^{*}-\varepsilon_{e l}^{*}}{\varepsilon_{p}^{*}+2 \varepsilon_{e l}^{*}}
$$

Here, $f_{C M}^{*}$ is the Clausius-Mossotti (CM) factor, relating the complex permittivity of a spherical homogeneous particle $\left(\varepsilon_{p}^{*}\right)$ to the environment $\left(\varepsilon_{e l}^{*}\right)$.

A biological cell is not a homogeneous particle, since the dielectric properties of the membrane are different from those of the cell interior. To include the dielectrical properties of both the membrane and the cell interior, biological cells are usually modelled by a core-shell model. The complex permittivity of a single-shelled spheroid is given by $[15,19]$ :

$$
\varepsilon_{p}^{*}=\varepsilon_{m e m}^{*} \frac{\gamma^{3}+2\left(\frac{\varepsilon_{i}^{*}-\varepsilon_{m e m}^{*}}{\varepsilon_{i}^{*}+2 \varepsilon_{m e m}^{*}}\right)}{\gamma^{3}-\left(\frac{\varepsilon_{i}^{*}-\varepsilon_{m e m}^{*}}{\varepsilon_{i}^{*}+2 \varepsilon_{m e m}^{*}}\right)} \text { with } \gamma=\frac{r+d_{m e m}}{r}
$$

in which $\varepsilon_{i}^{*}$ and $\varepsilon_{m e m}^{*}$ are the complex permittivities of the cytoplasm and membrane respectively, $r$ is the cell radius $(\mathrm{m})$ and $d_{\text {mem }}$ is the thickness of the cell membrane $(\mathrm{m})$. Usually, cells are modelled as single-shelled spheroids, although double-shell or multiple-shell models have been reported [20,21]. Now, the total impedance of the system (cells in suspension), based on the Maxwell-Wagner theory, can be described by (under the assumption of no field fringing) [15]:

\section{ECM with cell}

$$
Z_{\text {mix }}=\frac{K}{j \omega \varepsilon_{\text {mix }}^{*} \varepsilon_{0}}
$$

To model the presence of a cell in between two microelectrodes, the previously described ECM must be adapted. For simplification, the membrane resistance $\left(R_{m}\right)$ and the capacitance of the cell interior i.e. cytoplasm $\left(C_{i}\right)$ are often ignored $[7,15]$. By adding a membrane capacitance $\left(C_{m}\right)$ and cytoplasm resistance $\left(R_{i}\right)$ in parallel 

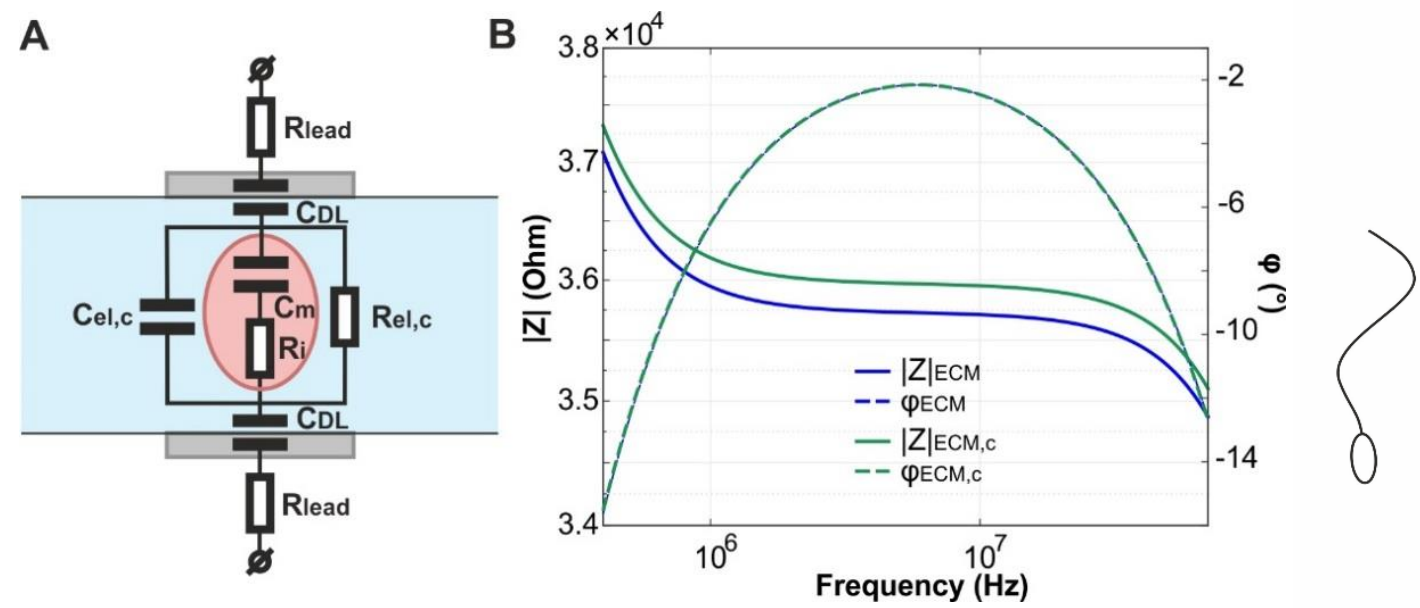

Fig. 3-3: A) ECM of a microfluidic chip with a cell in between a pair of microelectrodes. B) The impedance and phase response of the ECM of the microfluidic setup with (green) or without a cell (blue). Due to the insulating properties of the cell membrane in a high conductive environment, the simulated impedance of the ECM increased when including a cell in the model. The following simulation parameters were used: $w=s=l=20 \mu \mathrm{m}, A=4 \cdot 10^{-12} \mathrm{~m}^{2}, r$ $=2.5 \mu \mathrm{m}, d_{m}=5 \cdot 10^{-9} \mathrm{~m}, \sigma_{e l}=1.4 \mathrm{~S} \cdot \mathrm{m}^{-1}, \sigma_{i}=0.4 \mathrm{~S} \cdot \mathrm{m}^{-1}, \sigma_{m e m}=10^{-8} \mathrm{~s} \cdot \mathrm{m}^{-1} \cdot \varepsilon_{e l}=80$, $\varepsilon_{i}=60, \varepsilon_{\text {mem }}=11.3, C_{d}=20 \mu \mathrm{F} \cdot \mathrm{cm}^{-2}, C_{D L}=8 \cdot 10^{-8} \mathrm{~F}, R_{\text {lead }}=0 \Omega$.

with respect to the electrolyte resistance $\left(R_{e l, c}\right)$, an equivalent circuit is constructed of the microfluidic setup including cell (Fig. 3-3A). In this model, $C_{m}(\mathrm{~F})$ is calculated by $[7,16]$ :

$$
C_{m}=\frac{9 \varphi r C_{m, 0}}{4 K} \text { with } C_{m, 0}=\frac{\varepsilon_{m} \varepsilon_{0}}{d_{m}} \text { and } \varphi=\frac{V_{\text {cell }}}{V_{\text {elec }}}
$$

in which $C_{m, 0}$ is the specific membrane capacitance $\left(\mathrm{F} \cdot \mathrm{m}^{-2}\right)$. The volume fraction $\varphi$ describes the volume ratio of the cell with respect to electrolyte in between the two parallel-oriented electrodes $\left(w * s * l, \mathrm{~m}^{3}\right)$. The cytoplasm resistance is given by $[7,16]$ :

$$
R_{i}=\frac{4 K\left(\frac{1}{2 \sigma_{e l}}+\frac{1}{\sigma_{i}}\right)}{9 \varphi}
$$


The resistance $R_{e l, c}(\Omega)$ and capacitance $C_{e l, c}(\mathrm{~F})$ of the electrolyte need to be modified when modelling a cell in between the electrodes $[7,16]$ :

$$
\begin{gathered}
R_{e l, c}=\frac{K}{\sigma_{e l}\left(1-\frac{3 \varphi}{2}\right)} \\
C_{e l, c}=\frac{\varepsilon_{\infty}}{K} \text { with } \varepsilon_{\infty} \simeq \varepsilon_{e l} \varepsilon_{0}\left(1-3 \varphi\left(\frac{\varepsilon_{e l} \varepsilon_{0}-\varepsilon_{i} \varepsilon_{0}}{2 \varepsilon_{e l} \varepsilon_{0}+\varepsilon_{i} \varepsilon_{0}}\right)\right)
\end{gathered}
$$

The equivalent impedance of the whole circuit $Z_{E C M, c}$ can now be described as:

$$
Z_{E C M, c}=2 R_{\text {lead }}+Z_{\text {cell }}+\frac{2}{j \omega C_{D L}}
$$

Now, the equivalent model of the submerged cell $Z_{c e l l}$ is derived as:

in which $Y$ is described by:

$$
Z_{c e l l}=\frac{R_{e l, c}\left(1+j \omega R_{i} C_{m}\right)}{Y}
$$

$$
Y=j \omega R_{e l, c} C_{m}+\left(1+j \omega R_{e l, c} C_{e l, c}\right)\left(1+j \omega R_{i} C_{m}\right)
$$

The ECM and the electrical response of the microfluidic setup with cell are shown in Fig. 3-3A and Fig. 3-3B, respectively. Since $C_{m}$ and $C_{e l, c}$ have a large effect on the impedance, the simulated response is very similar to the response of the ECM without cell. When zooming-in on the resistive plateau (Fig. 3-3B), a small increase of the impedance is observed when introducing a cell in between the electrodes. In the high $\mathrm{kHz}$ range and low $\mathrm{MHz}$ range, the cell membrane acts as an insulator and blocks the flow of current. As a result, the impedance increased.

\subsection{Dielectrophoresis}

Besides the use of electrical fields for analysis, they can be employed for AC electrokinetic manipulation of cells. Dielectrophoresis is a well-known example of electrokinetic manipulation, which is used for applications including cell trapping (paragraph 2.3), focusing and separation [22,23]. DEP describes the force, which is the result of a field-induced dipole moment on a dielectric, polarizable particle when introduced to a non-uniform electric field [24]. This dipole moment arises from the accumulation of charge on the particle-electrolyte interface, i.e. interfacial 
polarization. This accumulation of charge is dependent on the movement of free charges, called conductive polarization, and the displacement of bound charge, called dielectric polarization. The two charging mechanisms can be modelled by a simple equivalent model: a resistor in parallel with a capacitor. At low frequencies the polarization is dominated by (free) ion conduction, while at high frequencies the polarization is determined by the particle's permittivity (3-17). As a result, the direction and magnitude of the induced dipole moment and the resulting DEP force depends on the frequency of the applied field. This frequency-dependent behaviour of a particle's polarization is described by the CM-factor (3-16), in which the particle is considered as a spherical, uniform particle. This factor describes the relation between the complex permittivities of the particle and the surrounding medium. Since a biological cell is modelled as single shelled spheroid, membrane properties have to be taken into account when calculating the particle's permittivity. The DEP force $\mathrm{F}_{D E P}(\mathrm{~N})$, exerted on a spherical particle, is described by [23]:

$$
\left\langle F_{D E P}\right\rangle=2 \pi \varepsilon_{e l} \varepsilon_{o} r^{3} \operatorname{Re}\left[f_{C M}^{*}\right] \nabla E_{r m s}^{2}
$$

in which $\operatorname{Re}\left[f_{C M}^{*}\right]$ is the real part of the CM-factor and $E_{r m s}$ is root-mean-square value of the applied electrical field (V).

The particle's conductivity and permittivity, compared to those of the surrounding medium, determine the sign of the $\mathrm{CM}$-factor and the corresponding direction of the DEP force. This force can either be repulsive (negative DEP or nDEP, Fig. 3-4A), pushing the particle away from high field gradients or attractive (positive DEP or pDEP, Fig. 3-4B), attracting the particle towards high field gradients. When subjecting a cell to a rotating electrical field, the sign of the imaginary part of the $\mathrm{CM}$-factor $\left(\operatorname{Im}\left[f_{C M}^{*}\right]\right)$ will determine if a cell will rotate with or against the direction of the field [15]. This phenomenon, called electrorotation, is not discussed in this overview and is described elsewhere in more detail [25].

Now consider a non-conducting particle with a low permittivity $\left(\varepsilon_{p}=2.3\right)$ compared to the surrounding non-conducting water $\left(\varepsilon_{e l}=79\right)$. The medium is more polarizable than the particle, resulting in a negative $\mathrm{CM}$-factor (Fig. 3-5 graph A). When the particle has a higher conductivity $\left(\sigma_{i}=0.01 \mathrm{~S} \cdot \mathrm{m}^{-1}\right)$ than the nonconducting water $\left(\sigma_{i}<0.01 \mathrm{~S} \cdot \mathrm{m}^{-1}\right)$, the $\mathrm{CM}$-factor is positive at low frequencies (Fig. 3-5 graph B). However, at higher frequencies the particle's permittivity starts to be dominant, resulting in shift to a negative $\mathrm{CM}$-factor and nDEP behaviour at higher frequencies. 

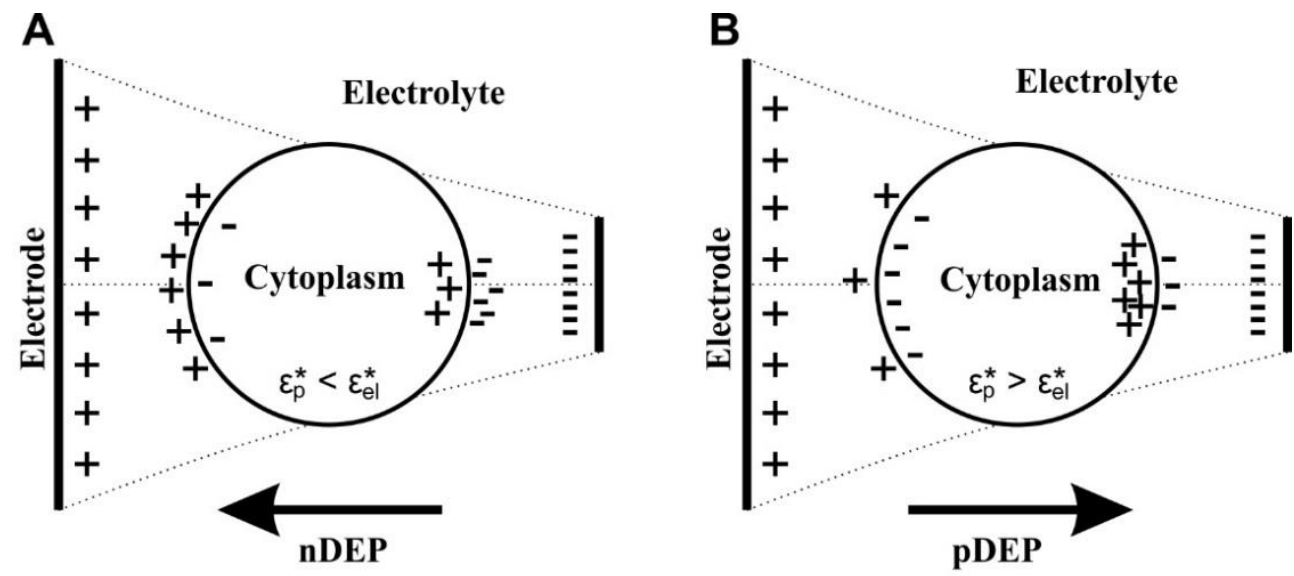

Fig. 3-4: A) A particle experiences a pDEP force when the particle's polarizability is higher compared to the polarizability of the medium. B) In case of higher medium polarizability, nDEP force is exerted on the particle.

Now, when a spherical cell $\left(\varepsilon_{p}=75, C_{m, 0}=1 \mu \mathrm{F} \cdot \mathrm{cm}^{-2}, \sigma_{i}=0.5 \mathrm{~s} \cdot \mathrm{m}^{-1}\right.$, specific membrane conductance $G_{m, 0}=5 \mathrm{~ms} \cdot \mathrm{cm}^{-2}$ ) is submerged in a low-conductive solution $\left(0.1 \mathrm{~S} \cdot \mathrm{m}^{-1}\right)$, two relaxations are observed in the spectrum, which are caused by the two interfaces within the system (Fig. 3-5 graph C): the medium-membrane and medium-cytoplasm interface [24]. Due to low membrane conductance at low frequencies, the $\mathrm{CM}$-factor is negative. When increasing the frequency $(\mathrm{MHz}$ range), the cell membrane is effectively short-circuited [24].

Besides manipulation of cells, DEP can be used for cell analysis. In practice, the measurement of the DEP force on a single particle is hard due to the confounding effects of Brownian motion and electrically induced fluid flow [15]. However, the crossover frequency can provide useful information upon the electrical properties of suspended particles as a function of electrolyte conductivity. The crossover frequency is defined as the frequency at which the cell does not experience a DEP force at which the real part of the $\mathrm{CM}$-factor is 0 . The crossover frequency $f_{\text {cross }}$ $(\mathrm{Hz})$ is calculated by [15]:

$$
f_{\text {cross }}=\frac{1}{\sqrt{2 \pi}} \sqrt{\frac{\sigma_{e l}-\sigma_{p}}{\varepsilon_{p}-\varepsilon_{e l}} f_{M W}}
$$



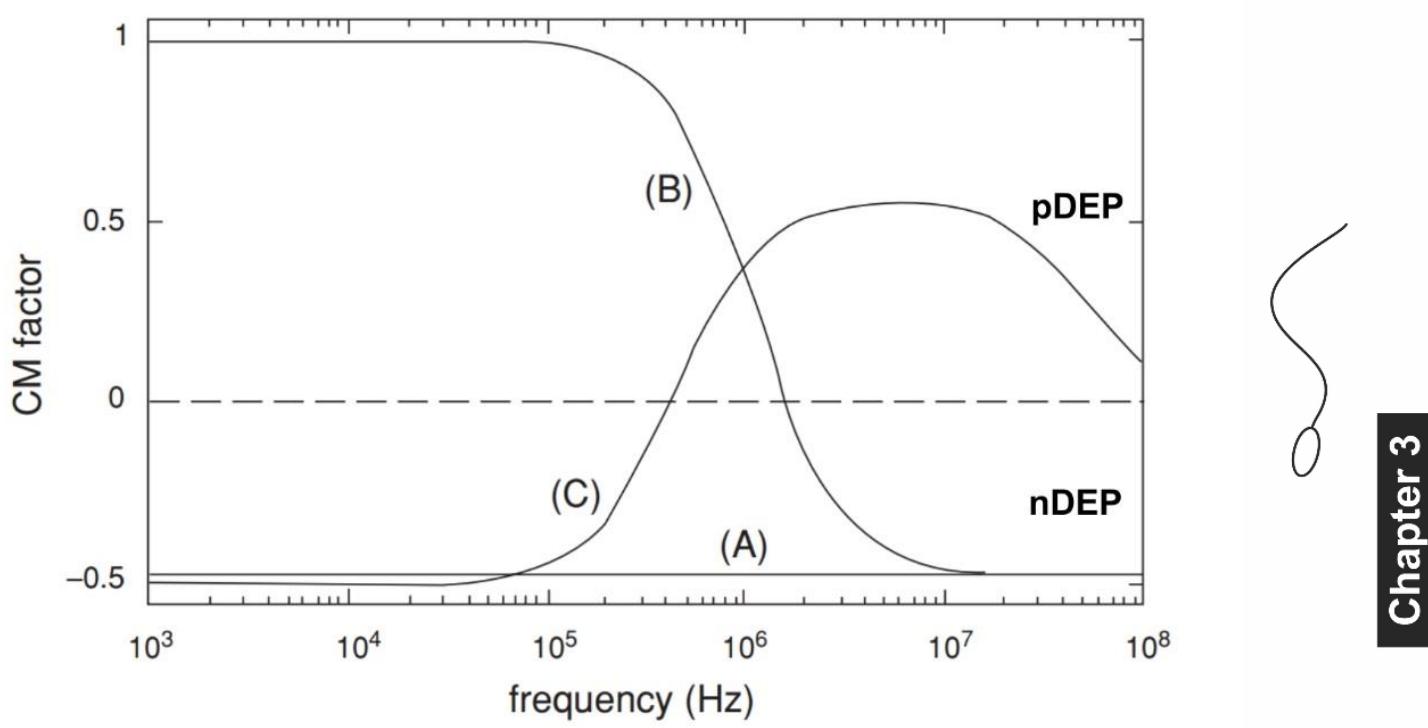

Fig. 3-5: Real part of the CM-factor as a function of frequency. A) The CMfactor for a non-conducting particle in a non-conducting medium is negative over the entire frequency range. B) Higher particle conductivity compared to the medium results in PDEP at low frequencies and nDEP at higher frequencies due to higher medium permittivity. C) A cell, modelled as a single-shelled spheroid, experiences two dispersions in a lowconductive buffer. At low frequencies, the cell is influenced by nDEP, whereas at intermediate frequencies, the cell is exposed to PDEP due to higher cytoplasm conductivity compared to the medium conductivity (adapted from [24]).

in which $f_{M W}$ describes the Maxwell-Wagner relaxation frequency $(\mathrm{Hz})$. This relaxation frequency is dependent on the Maxwell-Wagner time constant $\tau_{M W}(\mathrm{~s})$ [15]:

$$
f_{M W}=\frac{1}{2 \pi \tau_{M W}} \text { with } \tau_{M W}=\frac{\varepsilon_{p}+2 \varepsilon_{e l}}{\sigma_{p}+2 \sigma_{m}}
$$

When assuming the simplified single-shell spheroid model for a single cell in suspension, the crossover frequency can also be calculated by [15]:

$$
f_{\text {cross }}=\frac{\sqrt{2}}{8 \pi r C_{m, 0}} \sqrt{\left(4 \sigma_{e l}-r G_{m e m, 0}\right)^{2}-9 r^{2} G_{m e m, 0}^{2}}
$$


in which $G_{m e m, 0}$ is the specific cell membrane conductance $\left(\mathrm{S} \cdot \mathrm{m}^{-2}\right)$.

\subsubsection{Transmembrane potential}

Direct interaction of electrical fields with cells can negatively affect cell physiology. Especially, the interaction of the electrical field with cell membranes can cause cell damage. The electrical response of a cell membrane can be modelled by a parallel $\mathrm{RC}$ circuit, accounting for the membrane conductance and membrane capacitance [24]. At low frequencies ( $\mathrm{kHz}$ range), this circuit mainly acts as a resistor. Since the membrane conductance is low, a large potential drops across the cell membrane. When this induced potential, compared to a $-70 \mathrm{mV}$ resting potential, exceeds $0.2-$ $1.5 \mathrm{~V}[26,27]$, membrane electroporation can occur. At high frequencies (> $\mathrm{MHz}$ range), this voltage drop declines due to a decrease of the impedance of the cell membrane with increasing frequency $\left(Z_{C}=1 / j \omega C\right)$. The induced potential is also called transmembrane potential $\left|V_{T M}\right|(\mathrm{V})$ and is described by [24]:

$$
\left|V_{T M}\right|=\frac{1.5|E| r}{\sqrt{1+(\omega \tau)^{2}}}
$$

where $|E|$ is the maximal electrical field $\left(\mathrm{V} \cdot \mathrm{m}^{-1}\right), \tau$ is the polarization time constant (s) described by [24]:

$$
\tau=\frac{r C_{m, 0}\left(\rho_{\text {cyto }}+0.5 \rho_{e l}\right)}{1+r G_{m, 0}\left(\rho_{\text {cyto }}+0.5 \rho_{e l}\right)}
$$

in which $\rho_{c y t o}$ and $\rho_{e l}$ are the cytoplasm resistivity and electrolyte resistivity $(\Omega \cdot m)$, respectively. In general, high frequency nDEP excitation (> $10 \mathrm{MHz}$ ) using moderate field strengths ( $\approx 10^{\prime} \mathrm{s} \mathrm{kV} \cdot \mathrm{m}^{-1}$ range) does not inflict high transmembrane potentials and significant cell damage on cells in saline solutions [24].

\subsubsection{Joule heating}

Current-induced heating in microfluidic systems is a known side-effect when applying DEP for cell manipulation [24]. Especially, nDEP systems can suffer from Joule heating when using high conductive saline solutions. Induced temperature changes have a profound effect on the cell physiology. Temperature increase of $>$ $4{ }^{\circ} \mathrm{C}$ above physiological temperature $\left(37^{\circ} \mathrm{C}\right)$ results in rapid cell death, whereas increase of around $>1{ }^{\circ} \mathrm{C}$ is known to alter kinetic processes within the cell [24]. A 
temperature increase $<1{ }^{\circ} \mathrm{C}$ above physiological temperature is considered safe for mammalian cells [24].

\subsection{Application of microfluidic impedance cytometry}

Microfluidic impedance flow cytometry is a technique with which the electrical properties of single cells can be investigated. This technique utilizes integrated microelectrodes [28] for cell analysis and has several important advantages over traditional Coulter counting. With Coulter counting, DC is used to determine the resistance change upon particle transfer through a narrow aperture. This resistance change depends on the displacement of conductive fluid and therefore, on the volume of the particle. Contrarily, impedance cytometry can be performed using both DC and AC. By varying the field frequency, different aspects of a biological cells can be investigated [29]. At low frequencies, the polarized cell membrane acts as an insulator due to which the measured conductivity is related to the total cell volume (similar to Coulter counting). At intermediate frequencies, the polarization of the membrane decreases, allowing investigation of the cell membrane properties (membrane capacitance). In the high frequency region, minimal polarization occurs, allowing investigation of the cell interior (cytoplasm conductivity and permittivity) [7, 15]. Furthermore, performing microfluidic impedance cytometry using multiple frequencies simultaneously have shown valuable for discrimination of cell populations [7]. By defining the electrical response of single cells as electrical opacity, which is the ratio of measured impedance at a high frequency compared to a low frequency [7], the measurements are corrected for cell size and cell position in the microfluidic channel. This method have been extensively used for cell discrimination [30-33] and the analysis of cell viability [34, 35], morphology [21,36] and cell differentiation $[29,37]$.

\subsubsection{Cell discrimination}

Microfluidic impedance cytometry is a very suitable technique for cell discrimination based on cell size and electrical properties. A large number of reports have shown cell discrimination of a wide variety of cell types, including blood species (erythrocytes [30, 38-40], leukocytes [31, 41], platelets [42]), bacteria $[43,44]$, yeast $[21,36,45]$, algae [46], cancer cells [47-52], sperm cells [53] and stem cells $[37,54]$. A number of these papers is presented to provide a short overview of the application of microfluidic impedance cytometry. 


\section{Blood cells}

An early report on discrimination of cell types is reported by the group of Philippe Renaud [55]. They used a microchannel with two planar microelectrode pairs to record the current after applying a potential to the electrodes. They showed clear discrimination of erythrocytes and ghost cells when comparing the signal amplitudes at $1.72 \mathrm{MHz}$ and $15 \mathrm{MHz}$.

In a report from the same group [30], a microfluidic system with two pairs of parallel oriented microelectrodes (Fig. 3-6A) was used to distinguish erythrocytes from polystyrene beads using data recorded at $602 \mathrm{kHz}$ and $10 \mathrm{MHz}$. After current amplification and differential subtraction, the differential impedance was calculated (Fig. 3-6B). They used glutaraldehyde to fixate erythrocytes and showed the resulting effect on their electrical properties. Upon higher concentration of glutaraldehyde, the membrane permeability increased, showing increased opacity (Fig. 3-6C). Other research upon erythrocytes include work of Zheng et al. [40]. They used a constriction channel to record mechanical and electrical properties of erythrocytes. Changes in cell transit time, impedance amplitude and impedance phase at $100 \mathrm{kHz}$ were used to distinguish neonatal from adult erythrocytes.

The use of microfluidic impedance cytometry for discrimination of leukocytes is pioneered by the group of Hywel Morgan [31]. By performing differential impedance analysis on whole blood (after RBC lysis) in a microfluidic cytometer at 503 and $1707 \mathrm{kHz}$, neutrophils, monocytes and T-lymphocytes were successfully distinguished (Fig. 3-7A). These findings were verified using on- and off-chip fluorescent measurements. Furthermore, the obtained electrical data of different species was in good agreement with the simulated impedance response of the different leukocyte species, showing the effect of cell size and membrane capacitance on the absolute impedance. Moreover, their results were in good agreement with those obtained by conventional flow cytometric analysis and full blood analysis performed in the hospital (Fig. 3-7B) [31]. In a different paper from the same group, an integrated microfluidic system is reported that is able to count leukocytes in full blood [41]. In order to accurately count the amount of leukocytes, erythrocytes were lysed and removed on-chip. Recorded cell data showed high correlation with cell counts obtained with commercial haematology machines. 


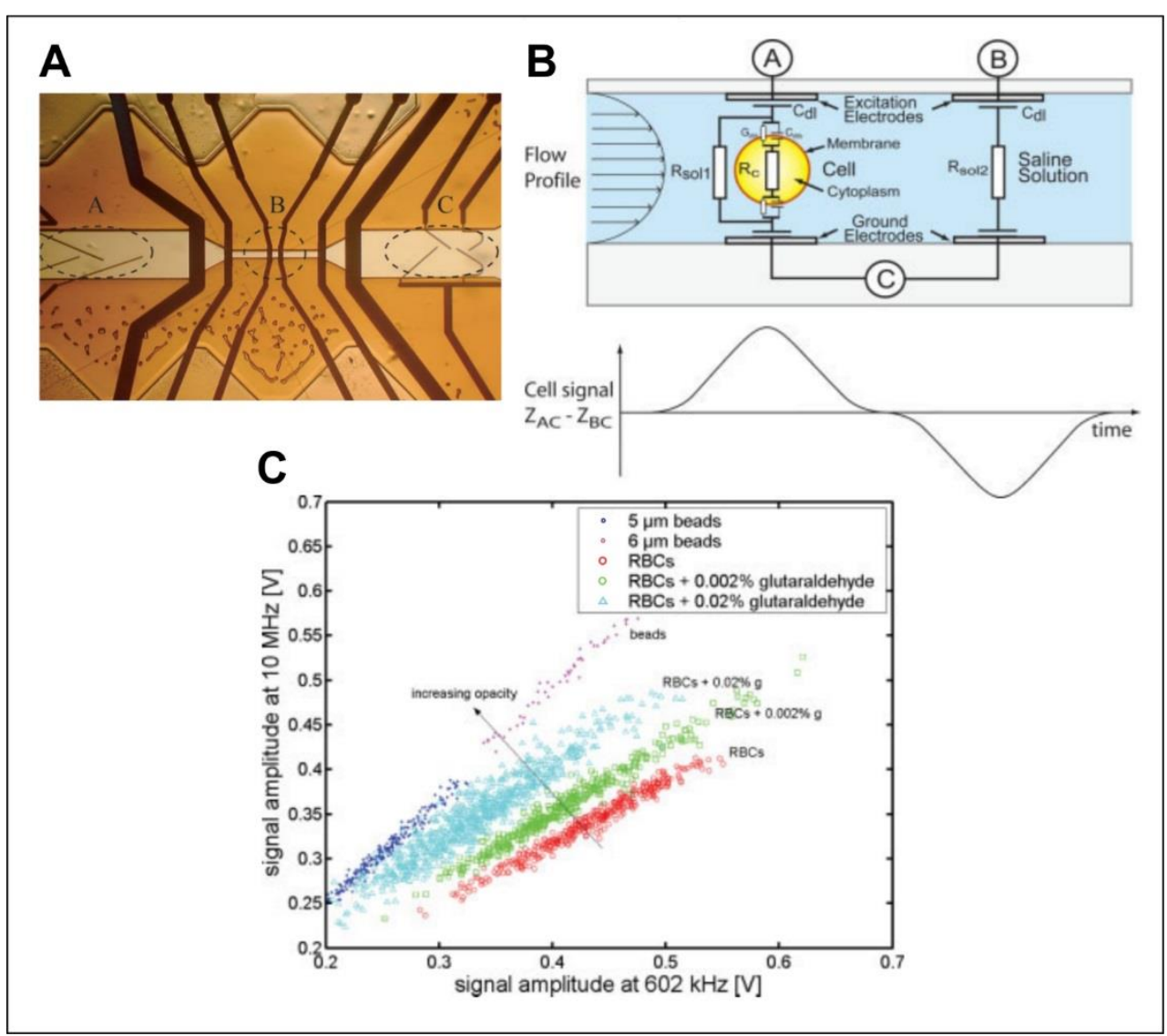

Fig. 3-6: Electrical analysis of erythrocytes. A) Erythrocytes were analysed in a channel constriction with parallel microelectrodes using B) differential impedance analysis at two frequencies. C) The impedance amplitude at $602 \mathrm{kHz}$ and $10 \mathrm{MHz}$ were used to distinguish erythrocytes from beads and to investigate the effect of glutaraldehyde (adapted from [30]).

Furthermore, they developed a point-of-care system for blood cell analysis, which combines sample preparation and cell analysis, allowing discrimination of red blood cells, platelets, granulocytes, lymphocytes and monocytes [56]. In subsequent work, they worked on the design of cytometers combining electrical with integrated optical components (Fig. 3-8A) [33, 57]. With these improved cytometers, cell analysis was performed using recorded side scattered light, fluorescence and impedance data [33]. 


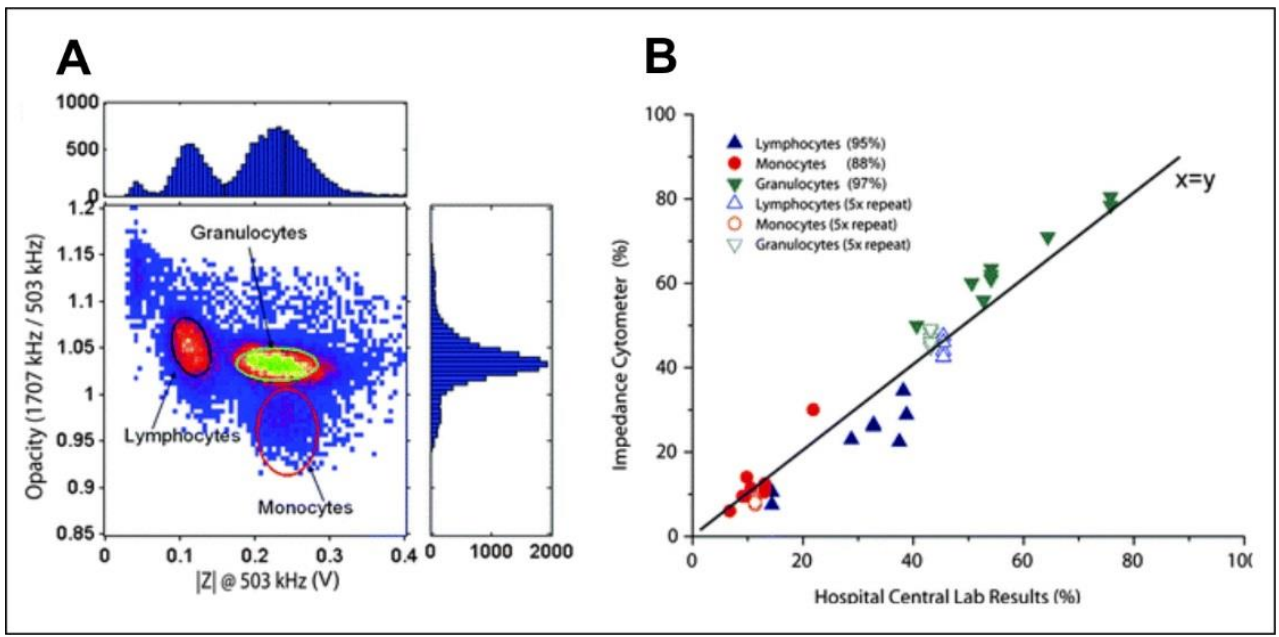

Fig. 3-7: Microfluidic impedance cytometry of leukocytes in blood. A) After differential measurements at $503 \mathrm{kHz}$ and $1707 \mathrm{kHz}$, the opacity and impedance magnitude at $503 \mathrm{kHz}$ were used to distinguish lymphocytes, granulocytes and monocytes. B) These results were in good agreement with those obtained by full blood analysis performed at the hospital using conventional flow cytometric analysis (adapted from [31]).

Besides the discrimination of monocytes from neutrophils based on differences in cell membrane capacitance, which was already established in their previous research, this cytometer was able to qualitatively discriminate CD4+ and CD4lymphocytes (important for AIDS monitoring) based on fluorescent analysis (Fig. 3-8B). Obtained blood counts were in good agreement with measurements obtained with a state of the art fluorescence-activated cell sorting (FACS) machine, further emphasizing its potential to be used as an alternative technique for haematology analysis (in remote areas) [33].

\section{Bacteria}

Impedance cytometry has also been exploited for the analysis of bacteria. Bernabini et al. [43] showed the detection of bacteria and micron-sized particles using two parallel orientated microelectrode pairs at a measurement frequency of $503 \mathrm{kHz}$. To increase the sensitivity of the system, beads and bacteria were hydrodynamically focused in between two layers of insulating fluid [43]. Furthermore, Haandbaek et al. [44] used a single electrode pair with an incorporated resonator to increase the sensitivity of their cytometer. 


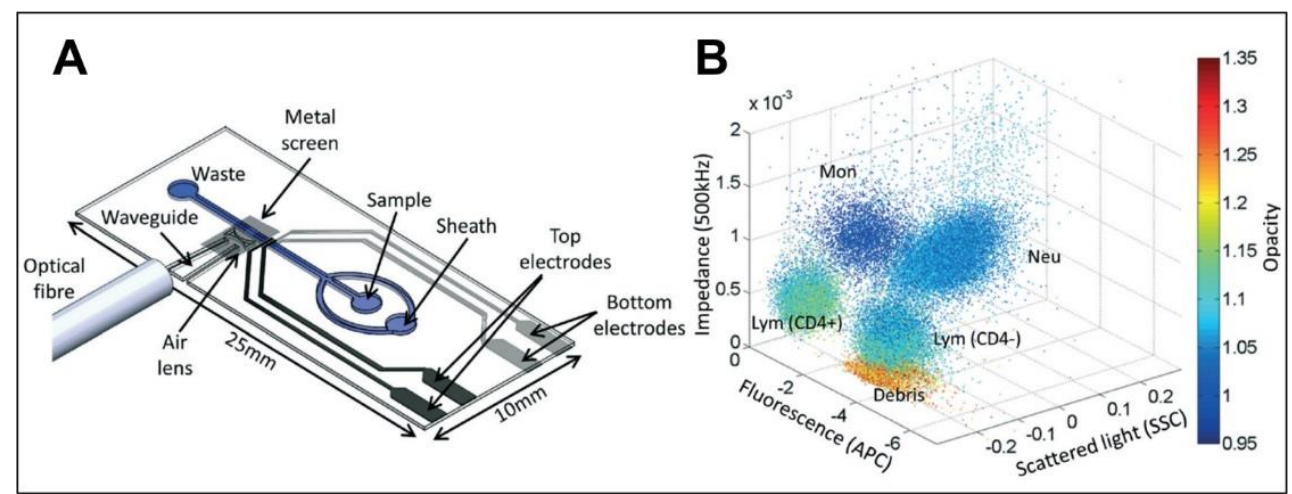

Fig. 3-8: A combined approach of impedance cytometry with integrated optical analysis. A) By integrating an optical fibre in an impedance cytometer, cell analysis was performed using measurements of the impedance, fluorescence and side scatter. B) Using this approach, monocytes, neutrophils and lymphocytes were distinguished, and moreover, CD4+ lymphocytes and CD4lymphocytes could be discriminated by fluorescence (adapted from [57]).

In combination with DEP focusing of particles and bacteria, their cytometer was able to distinguish beads from bacteria with similar size. Furthermore, they showed discrimination of $E$. coli and $B$. subtilis by means of a phase shift in current at measurement frequencies of 87.2 and $89.2 \mathrm{MHz}$ [44].

\section{Cancer cells}

Cancer cells have also been investigated using microfluidic impedance cytometry. A relative new development is the use of constriction channels, which dimensions are typically smaller compared to a cell. Besides analysis of the electrical properties of a cell, mechanical properties can be investigated, simultaneously. Kang et al. [48] used a cell constriction channel with integrated, interdigitated electrodes to distinguish normal (MCF-10A) from cancerous human breast cells (MCF-7). Impedance analysis was performed at 6 discrete frequencies at an amplitude of $0.25 \mathrm{~V}$. The two populations were distinguished using differences in impedance magnitude (real part) and phase angle at $500 \mathrm{kHz}$ [48]. Zheng et al. [58] used a similar approach to monitor the impedance response of human acute myeloid leukaemia cells (AML-2) and promyelocytic leukaemia cells (HL-60) at 7 discrete frequencies between 1 and $400 \mathrm{kHz}$ at $100 \mathrm{mV}$ using two $\mathrm{Ag} / \mathrm{AgCl}$ electrodes. Using the electrical data and data upon cell elongation, the cell populations could be 
distinguished. Differences in specific cell membrane capacitance and cytoplasm conductivity between both populations were explained by differences in cell membrane morphology and the nucleus-cytoplasm volume ratio, respectively [58]. In a similar approach by Zhao et al. [49], the constriction channel method was used to characterize the specific cytoplasm conductivity and membrane capacitance of two lung cancer cell lines using measurements at 1 and $100 \mathrm{kHz}$. In follow-up research [50], they were able to measure significant difference between high- and low-metastatic carcinoma cell strains 95D. When downregulating the oncogene membrane-associated protein CCNY in 95D cells, a statistically significant change was found in the specific membrane capacitance whereas no changes in cytoplasm conductivity were recorded. When downregulating the oncogene cytosol protein A549, only the cytoplasm conductivity significantly changed whereas the specific membrane capacitance did not change significantly [50].

\subsubsection{Cell viability}

Besides identification of different cell types based on their electrical properties, cellular information regarding viability can be extracted from electrical data. A good example is reported by Pierzchalski et al. [59]. They used an impedance flow cytometer to record impedance changes of passing MCF-7 cells. At $4 \mathrm{MHz}$, they distinguished viable from dead cells by comparing the recorded phase shift with the impedance amplitude. At $6 \mathrm{MHz}$, a further distinguishment was made between apoptotic cells and necrotic cells (Fig. 3-9A) [59]. Similar results were reported by Gou et al. [60]. They fabricated a PDMS device with transfer printed gold electrodes and used this device to distinguish viable, apoptotic and necrotic human liver carcinoma cells based on changes in resistance and capacitance measured at 100 $\mathrm{kHz}$. In a report of David et al. [34] the viability and membrane potential of bacteria (Bascillus megaterium) were investigated. A clear difference between viable and heat-inactivated cells was observed by a difference in recorded phase angle at 10 $\mathrm{MHz}$ (Fig. 3-9B). Furthermore, using multi frequency analysis at 0.5 and $10 \mathrm{MHz}$, they discriminated cells in exponential growth from cells in static growth state [34]. In a comprehensive report of Cheung et al. [29], showing the use of microfluidic impedance cytometry for multiple applications including differential blood cell count, analysis of cell dead, changes in membrane potential and internal calcium concentration, they reported upon the discrimination of dead and viable yeast cells (Saccharomyces cerevisiae) and bacteria (Bacillus megaterium) by a difference in recorded phase angle at $10 \mathrm{MHz}$ [29]. 


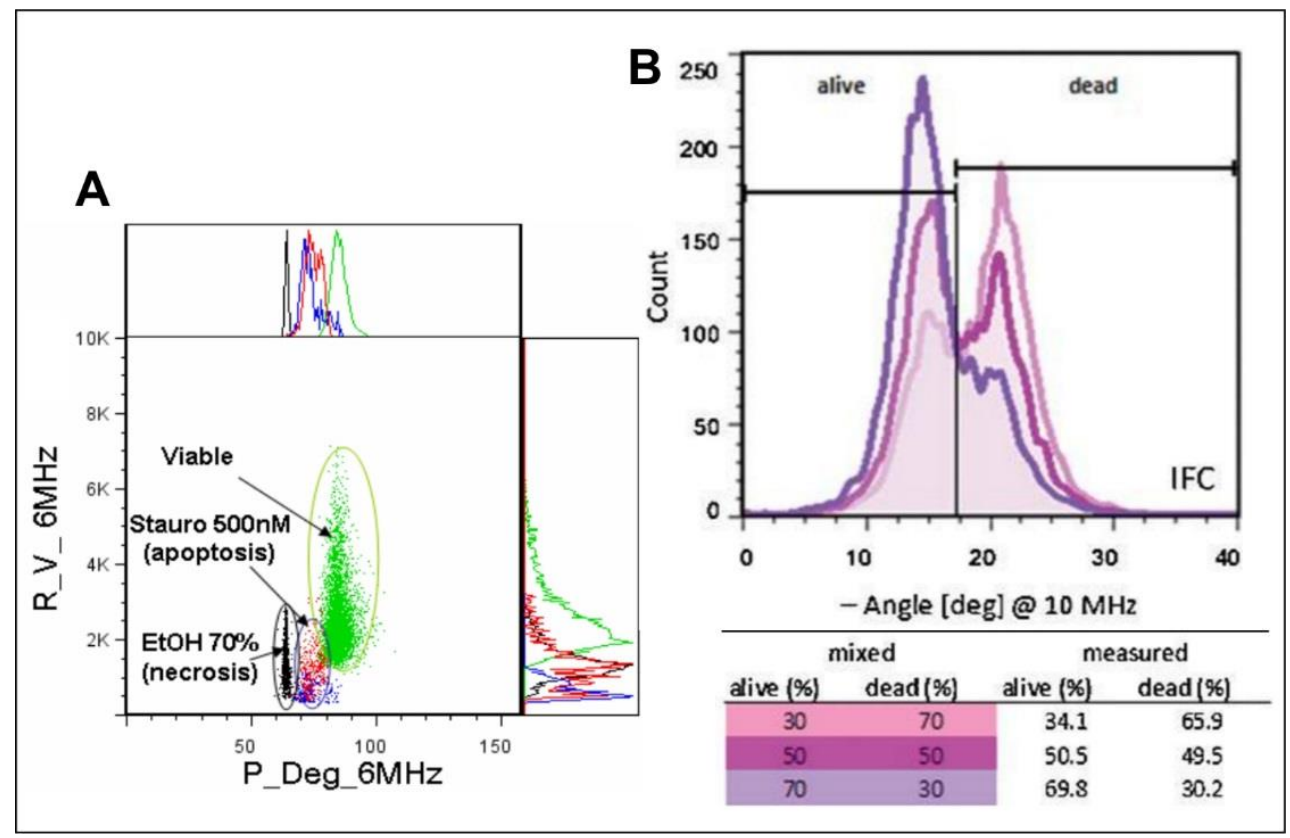

Fig. 3-9: Analysis of cell viability using microfluidic impedance cytometry. A) Pierzchalski et al. [59] distinguished viable, apoptotic and necrotic MCF-7 cells by the impedance magnitude and phase after measurements at a frequency $6 \mathrm{MHz}$. B) Similarly, David et al. [34] discriminated viable and dead cells base on the phase angle of measurement recorded at $10 \mathrm{MHz}$ (adapted from [34, 59]).

\subsubsection{Cell morphology}

Few reports show the use of microfluidic impedance cytometry for the analysis of cell morphology. Haandbæk et al. [21] showed the discrimination of a wild-type yeast strain from a mutant yeast strain based on intracellular vacuole content. A difference in vacuole content resulted in a difference in recorded opacity at measurement frequencies of 0.5 and $100 \mathrm{MHz}$. These experiments were in agreement with finite element method (FEM) simulation of the opacity of the wild type (triple shell model) and mutant yeast strain (double shell model) [21]. Shaker et al. [36] developed a cytometer specifically designed for cell morphology discrimination. Four electrodes oriented in a cross configuration were used to record the impedance in orthogonal orientations in the sensing area of their device using measurement frequencies of 427 and $533 \mathrm{kHz}$ in the horizontal and vertical direction, respectively. In front of the sensing area, a number of lateral electrodes 
were used to accurately position the cells in the middle of the channel using nDEP. As a proof of concept, they showed the ability to distinguish between division stage of budding yeast cells (S-phase and M-phase) based on cell shape anisotropy [36].

\subsubsection{Cell differentiation}

Cell differentiation was investigated by Song et al. [37]. They used a microfluidic PDMS device with two integrated micro-apertures to measure the change in impedance using external electrodes upon passage of mice embryonic carcinoma cells (P19). The recorded signal upon cell passage in the first aperture was corrected for the signal of the second aperture to calculate the differential response. After multi frequency analysis at $50 \mathrm{kHz}$ and $1 \mathrm{MHz}$, the opacity showed a clear difference between differentiated and undifferentiated P19 cells [37]. Another example of cytometric analysis of cell differentiation is reported by Cheung et al. [29]. They measured induced HL-60 cell differentiation into macrophages by impedance flow cytometry measurements, observing a shift in recorded phase angle of differentiated cells at $8 \mathrm{MHz}$ [29].

\subsubsection{Cell disease}

Microfluidic impedance cytometry was also employed for detection of cell disease and abnormalities. Kuttel et al. [39] reported upon the discrimination of normal and Babesia bovis infected erythrocytes. After analysis at $8.7 \mathrm{MHz}$ in low conductive buffer $\left(0.56 \mathrm{~ms} \cdot \mathrm{cm}^{-1}\right)$, infected erythrocytes could be distinguished by a shift in phase angle [39]. In similar work, Du et al. [38] studied erythrocyte infection with a malaria parasite (Plasmodium falciparum) using impedance cytometry at measurement frequency of $2 \mathrm{MHz}$. Furthermore, they investigated the several infection stages by means of changes in electrical properties [38].

\subsection{Application of microfluidic impedance spectroscopy}

Microfluidic electrical impedance spectroscopy is a technique in which the electrical response of a single cell or multiple cells is recorded over a broad frequency range in a microfluidic setting. To perform a spectroscopic measurement on a single cell, which typically requires a longer analysis time with respect to analysis using impedance cytometry, the cell must be (temporarily) retained on a fixed position with respect to the microelectrodes. Therefore, these platforms require integrated cell trapping features. Furthermore, many reports on microfluidic impedance spectroscopy of single cells show the use of single electrode pairs for the analysis of entrapped cells. The recorded impedance is affected by 
systematic effects (e.g. double layer and parasitic capacitance) due to which theoretical modelling and data fitting is necessary to obtain information upon the dielectric properties of the cell cytoplasm and membrane. Single cell impedance spectroscopy has a low measurement throughput compared to impedance cytometry. Therefore, impedance spectroscopy is less suitable for analysis and discrimination of cell populations. However, impedance spectroscopy is very suitable to study single cell events over time, such as cell shrinkage and membrane permeation due to drug or toxins exposure.

\subsubsection{Electrical characterization of cancer cells}

Most reports upon single cell impedance spectroscopy focus on the analysis of cancer cells. Discrimination of cancer cells from normal cells is usually reported by differences in specific cytoplasm conductivity and specific membrane capacitance. Various cell trapping methods were used, including hydrodynamic traps, blocking microstructures and DEP traps.

Han et al. [62] showed the isolation of breast cancer cells (MDA and MCF) from blood after magnetically excluding red blood cells from the sample. After isolation, breast cancer cells were entrapped by suction in micro-hole traps and analysis was performed using electrodes at opposite sides of the micro-hole (top and bottom channel) between $100 \mathrm{~Hz}$ and $3 \mathrm{MHz}$. Using recorded impedance data, cancer cells were distinguished from normal cells based on cytoplasm resistance and membrane capacitance. Moreover, the electrical data was used to investigate the pathological stage of the trapped cancer cell [62]. In later work, they developed an alternative design for cell analysis, consisting of an in-channel cavity for cell entrapment [63].

Cho et al. [64] hydrodynamically entrapped cancer cells using perpendicular side channels. Integrated gold planar microelectrode were used to record the impedance between $40 \mathrm{~Hz}$ and $10 \mathrm{MHz}$ at $500 \mathrm{mV}$. Using their device, a difference between highly and poorly metastatic cancer cells was observed by a difference in phase shift at frequencies between $50 \mathrm{kHz}$ and $2 \mathrm{MHz}$. This observation was explained by differences in morphology, distinct protein expression and genetic alteration between both cell lines [64].

Jang and Wang [65] reported upon the entrapment of HeLa cells using micropillars. Using gold integrated electrodes, the impedance of these cells were measured with respect to the low-conductive, isotonic medium (conductivity not reported) at frequencies between 1 and $100 \mathrm{kHz}$ at 0.1 or $1.5 \mathrm{~V}$. Due to the conducting 
properties of the HeLa cells with respect to the low-conducting isotonic medium, a decrease in impedance was observed upon cell entrapment. Simulations were fitted to the measured data to calculate the cell capacitance and resistance [65].

Mondal et al. [66] reported upon the design of electrode microtraps, which were used for cell trapping and impedance analysis simultaneously, avoiding the necessity to design and align the microstructures with an electrode array. Two different electrodes (parallel and elliptical geometry) were used to record impedance changes of entrapped HeLa cells between frequencies of $100 \mathrm{~Hz}$ to 100 $\mathrm{kHz}$ at $0.1 \mathrm{~V}$. The data was fitted to an ECM to obtain values for the cytoplasm resistance and membrane capacitance [66].

Nguyen et al. [67] reported upon the use of SU-8 microstructures to capture cells. Gold microelectrodes were used for the analysis of adhering cancer cells on the surface of (functionalized) microelectrodes using impedance spectroscopy at a frequency range of $100 \mathrm{~Hz}$ to $1 \mathrm{MHz}$ at $10 \mathrm{mV}$ excitation. Using this platform, they analysed the migration of metastatic MDA-MB-231 cells and showed a high change in impedance magnitude of these cells compared to less-metastatic MCF-7 cells [68].

Lan and Jang $[69,70]$ designed a microfluidic platform, in which HeLa cells were moved and trapped by a combination of electrothermal and nDEP forces. Using their platform, they reported upon the differentiation of various cancer cells using impedance analysis between $20-101 \mathrm{kHz}$ at potentials of $0.2-1 \mathrm{~V}$ [71]. The cytoplasm resistance and membrane capacitance were obtained by curve-fitting the measurements to a theoretical model. In a later report, they optimized their device to transport, entrap and analyse HeLa cells, suspended in a sucrose solution, in SU-8 cavities [72].

\subsubsection{Toxin \& drug exposure}

The potential to monitor single cells over time makes electrical impedance spectroscopy a suitable technique to investigate the effects of toxins and drugs on the single cell level. A good example is reported by Malleo et al. [73]. They reported upon continuous differential impedance analysis of HeLa cells, which were trapped by SU-8 microstructures. Differential measurements were obtained by correcting the recorded impedance of a captured cell with the recorded impedance of a reference electrode pair. 


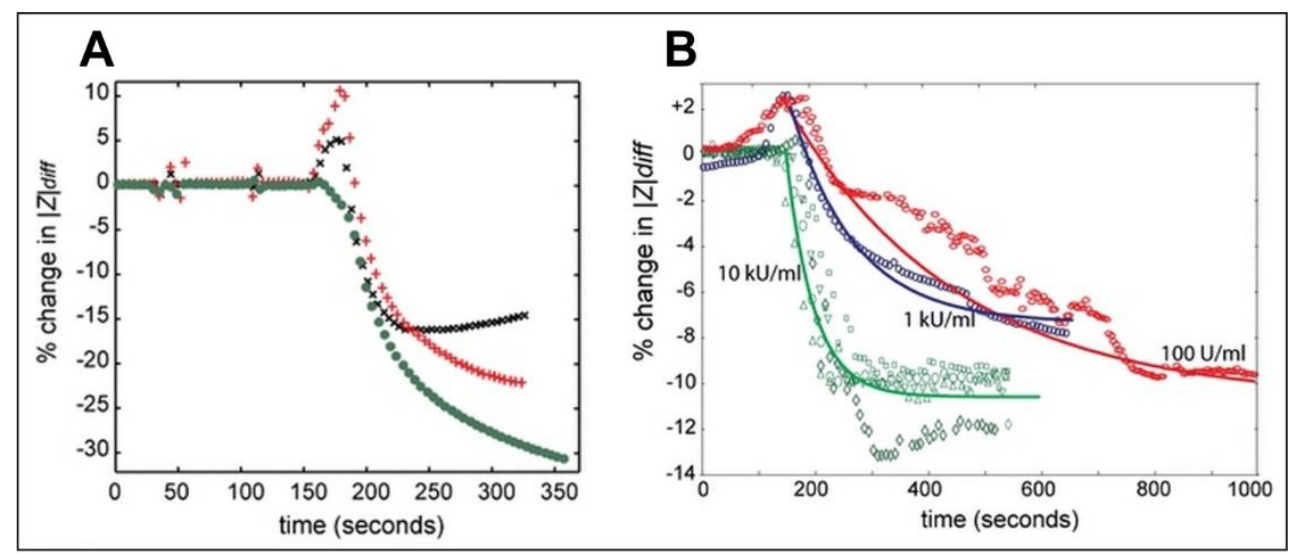

Fig. 3-10: Differential impedance response of entrapped HeLa cells when altering the cell membrane. A) Exposure to the detergent Tween-20, resulted in initial cell swelling, followed by membrane rupture, resulting in a decrease in differential impedance at 300 $\mathrm{kHz}$. B) Exposure to pore-forming toxin Streptolysin-O resulted in a decrease in impedance, proportionally to the toxin concentration (adapted from [73]).

Upon cell entrapment, an increase of 20-30\% in differential impedance magnitude was observed when continuously monitoring at $300 \mathrm{kHz}$ [73]. Exposing entrapped cells to the surfactant Tween-20 resulted in a concentration-dependent decrease of the differential impedance magnitude (Fig. 3-10A), which is explained by a gradual break-down of the cell membrane. Furthermore, exposure to the poreforming toxin Streptolysin-O resulted in decay of the impedance (Fig. 3-10B), in which the decay time constant was found to be inversely proportional to the toxin concentration [73].

Cho and Thielecke [74] used a micro-hole trap to capture mouse fibroblasts (L929) and monitor cell growth using impedance spectroscopy through-hole at frequencies between $1 \mathrm{~Hz}$ and $1 \mathrm{MHz}$ at $10 \mathrm{mV}$ with external electrodes in a 4-point measurement arrangement. Cell growth resulted in an increase in impedance magnitude at frequencies up to $10 \mathrm{kHz}$. Furthermore, they investigated the toxic effect of dimethylsulfoxide (DMSO) on entrapped cells using electrical measurements. The toxic effect resulted in a decrease in impedance magnitude and increase of phase (towards zero) over time at a measurement frequency of $1 \mathrm{kHz}$ [74]. In a report of Kurz et al. [75] from the same group, they optimized the microhole chip system to analysis sub-toxic effects on cells by polymer-DNA complexes, 


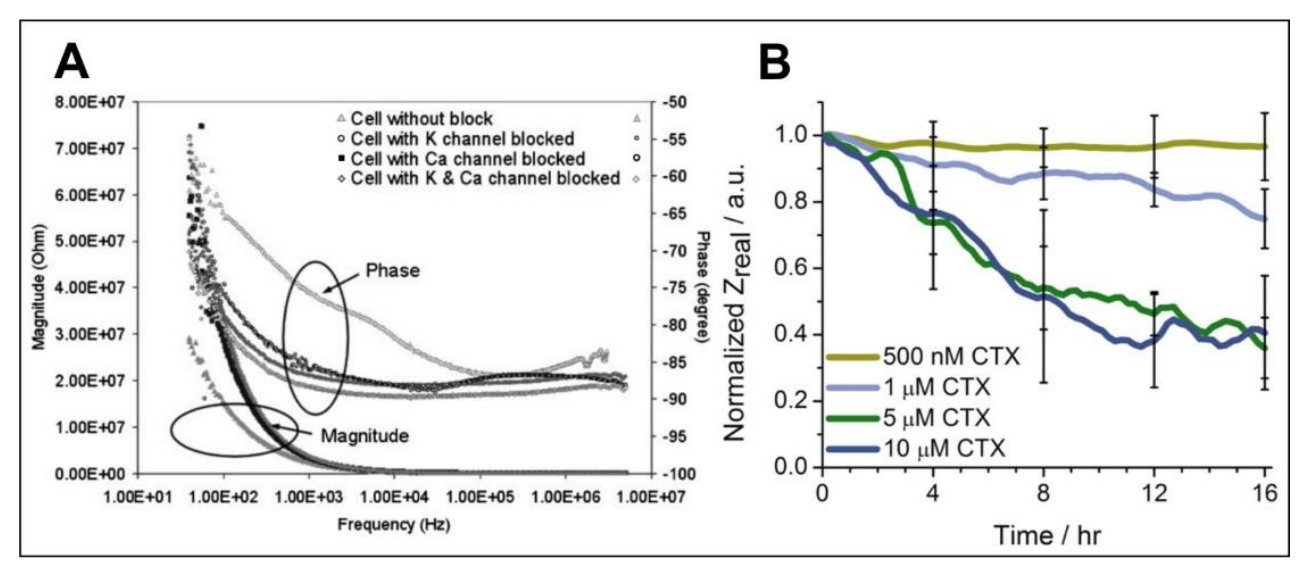

Fig. 3-11: The effect of blocked ion channels on the electrical impedance. A) Han and Frazier [76] reported upon altering the electrical properties of a single cell by blocking its $\mathrm{K}^{+}$and $\mathrm{Ca}^{2+}$ ion channels, resulting in an increased membrane capacitance and a decreased membrane conductance. B) Asphahani et al. [77] used CTX as an ion-channel blocking agent and observed a decrease in impedance due to CTX-induced morphological change of the cancer cells (adapted from $[76,77])$.

typically used for DNA transfection. Upon cell permeation, verified by PI staining, a decrease of the impedance magnitude was measured at $1 \mathrm{kHz}$ over time [75].

\subsubsection{Ion channel blockage}

Blocking of ion channels alters the electrical response of the cell membrane. Han and Frazier [76] reported upon the analysis of ion channels of entrapped bovine chromaffin cells. These cells were entrapped in micro-holes by applying a negative pressure over the small apertures using underlying microfluidic channels. The impedance was measured over a frequency range of $100 \mathrm{~Hz}$ to $5 \mathrm{MHz}$. By blocking the $\mathrm{K}^{+}$and $\mathrm{Ca}^{2+}$ ion channels, an increase of signal magnitude and a decrease in phase was observed (Fig. 3-11A). Ion channel blocking showed an increase in membrane capacitance and resistance.

Asphahani et al. [77] patterned human glioblastoma cells (U87MG) on peptidemodified gold microelectrode arrays. After cell seeding, impedance analysis was performed using $30 \mu \mathrm{m}$ and $250 \mu \mathrm{m}$ diameter electrodes under single cell or multiple cell conditions at frequencies between $500 \mathrm{~Hz}$ and $20 \mathrm{kHz}$ at $5 \mathrm{mV}$. Using the $30 \mu \mathrm{m}$ electrodes, they showed a decrease in normalized impedance magnitude upon exposure to ion channel inhibitor chlorotoxin (CTX) in a dose-dependent 
manner (Fig. 3-11B). Impedance changes were caused by the morphological changes of the cancer cells in response to CTX [77].

\subsection{Conclusions}

Impedance cytometry and spectroscopy are label-free and non-invasive techniques which are suitable for the analysis of single cells. Depending on the selected field frequency, different aspects of the cell can be investigated. However, the intrinsic electrical properties of the microfluidic system have to be taken into account when selecting this frequency. Intermediate frequencies between the high $\mathrm{kHz}$ range and low $\mathrm{MHz}$ range, at which the total system impedance is predominantly determined by the electrolyte resistance are most suitable for cell analysis. At these frequencies, cell characteristics such as cell volume, membrane capacitance and cytoplasm resistance can be investigated.

Impedance measurements have been used for a wide range of applications. Microfluidic impedance cytometry has been applied for the discrimination of cell populations based on cell type, disease state, morphology and differentiation [21, 29-37]. Various approaches have improved the sensitivity of micro-cytometers, including the use of differential methods at multiple frequencies [30, 31], the use of flow focusing with non-conducting fluids to reduce the measurement volume [43] and the integration with optical analysis [33, 57]. Microfluidic impedance spectroscopy was predominantly used to distinguish cancer cells from normal cells and to study the effects of ion channel blockage and exposure to toxins and drugs on single cells. Therefore, due to a high measurement throughput $\left( \pm 1000\right.$ s cells $\left.\cdot \mathrm{s}^{-1}\right)$ [33], impedance cytometry is more suitable to distinguish cell populations, whereas impedance spectroscopy can better be used to investigate changes in electrical properties of (single) cells over time.

\subsection{References}

1. Coulter, W.H., High speed automatic blood cell counter and cell analyzer. Proc Natl Electron Conf, 1956. 12: p. 1034-40.

2. Hoffman, R.A. and W.B. Britt, Flow-system measurement of cell impedance properties. J Histochem Cytochem, 1979. 27(1): p. 234-40.

3. Hoffman, R.A., T.S. Johnson, and W.B. Britt, Flow cytometric electronic direct current volume and radiofrequency impedance measurements of single cells and particles. Cytometry, 1981. 1(6): p. 377-84. 
4. Larsen, U.D., G. Blankenstein, and J. Branebjerg, Microchip Coulter particle counter. Transducers 97 - 1997 International Conference on Solid-State Sensors and Actuators, Digest of Technical Papers, Vols 1 and 2, 1997: p. 1319-22.

5. Foster, K.R. and H.P. Schwan, Dielectric properties of tissues and biological materials: a critical review. Crit Rev Biomed Eng, 1989. 17(1): p. 25-104.

6. Feldman, Y., A. Puzenko, and Y. Ryabov, Dielectric Relaxation Phenomena in Complex Materials. Fractals, Diffusion, and Relaxation in Disordered Complex Systems, Part A, 2006. 133: p. 1-125.

7. Sun, T. and H. Morgan, Single-cell microfluidic impedance cytometry: $a$ review. Microfluid Nanofluid, 2010. 8(4): p. 423-43.

8. Heileman, K., J. Daoud, and M. Tabrizian, Dielectric spectroscopy as a viable biosensing tool for cell and tissue characterization and analysis. Biosens Bioelectron, 2013. 49: p. 348-59.

9. Bard, A.J. and L.R. Faulkner, Electrochemical methods: fundamentals and applications. 2nd edition ed. 2001: John Wiley \& Sons, Inc.

10. Srinivasan, S., Electrode/electrolyte interfaces: stucture and kinetics of charge transfer, Fuel Cells. 2006, Springer US. p. 27-92.

11. Monk, P.M.S., Fundamentals of electroanalytical chemistry 2001, Chichester: John Wiley \& Sons Ltd.

12. Segerink, L.I., Fertility chip: a point-of-care semen analyser. 2011, University of Twente: Enschede.

13. Jacobs, P., A. Varlan, and W. Sansen, Design Optimization of Planar Electrolytic Conductivity Sensors. Med Biol Eng Comput, 1995. 33(6): p. 80210.

14. Olthuis, W., W. Streekstra, and P. Bergveld, Theoretical and ExperimentalDetermination of Cell Constants of Planar-Interdigitated Electrolyte Conductivity Sensors. Sensor Actuat B-Chem, 1995. 24(1-3): p. 252-6.

15. Morgan, H., et al., Single cell dielectric spectroscopy. J Phys D Appl Phys, 2007. 40(1): p. 61-70.

16. Sun, T., C. Bernabini, and H. Morgan, Single-colloidal particle impedance spectroscopy: complete equivalent circuit analysis of polyelectrolyte microcapsules. Langmuir, 2010. 26(6): p. 3821-8.

17. Pauly, H. and H.P. Schwan, Uber die Impedanz einer Suspension von kugelförmigen Teilchen mit einer Schale. Z. Naturforsch, 1959. 14b(125): p. 125-31.

18. Maxwell, J., A treatise on electricity and magnetism. Vol. 1. 1873: Oxford: Clarendon Press.

19. Valero, A., T. Braschler, and P. Renaud, A unified approach to dielectric single cell analysis: impedance and dielectrophoretic force spectroscopy. Lab Chip, 2010. 10(17): p. 2216-25. 
20. Asami, K., Characterization of biological cells by dielectric spectroscopy. J Non-Cryst Solids, 2002. 305(1-3): p. 268-77.

21. Haandbaek, N., et al., Characterization of subcellular morphology of single yeast cells using high frequency microfluidic impedance cytometer. Lab Chip, 2014. 14(2): p. 369-77.

22. Cetin, B. and D.Q. Li, Dielectrophoresis in microfluidics technology. Electrophoresis, 2011. 32(18): p. 2410-2427.

23. Khoshmanesh, K., et al., Dielectrophoretic platforms for bio-microfluidic systems. Biosens Bioelectron, 2011. 26(5): p. 1800-14.

24. Voldman, J., Dielectrophoretic Traps for Cell Manipulation, in BioMEMS and Biomedical Nanotechnology, M. Ferrari, R. Bashir, and S. Wereley, Editors. 2007, Springer US. p. 159-186.

25. Foster, K.R., F.A. Sauer, and H.P. Schwan, Electrorotation and levitation of cells and colloidal particles. Biophys J, 1992. 63(1): p. 180-90.

26. Weaver, J.C., Electroporation: a general phenomenon for manipulating cells and tissues. J Cell Biochem, 1993. 51(4): p. 426-35.

27. Rae, J.L. and R.A. Levis, Single-cell electroporation. Pflugers Arch, 2002. 443(4): p. 664-70.

28. Ayliffe, H.E., A.B. Frazier, and R.D. Rabbitt, Electric impedance spectroscopy using microchannels with integrated metal electrodes. J Microelectromech S, 1999. 8(1): p. 50-7.

29. Cheung, K.C., et al., Microfluidic impedance-based flow cytometry. Cytometry A, 2010. 77(7): p. 648-66.

30. Cheung, K., S. Gawad, and P. Renaud, Impedance spectroscopy flow cytometry: on-chip label-free cell differentiation. Cytom Part A, 2005. 65(2): p. 124-32.

31. Holmes, D., et al., Leukocyte analysis and differentiation using high speed microfluidic single cell impedance cytometry. Lab Chip, 2009. 9(20): p. 28819.

32. Schade-Kampmann, G., et al., On-chip non-invasive and label-free cell discrimination by impedance spectroscopy. Cell Prolif, 2008. 41(5): p. 83040.

33. Spencer, D., G. Elliott, and H. Morgan, A sheath-less combined optical and impedance micro-cytometer. Lab Chip, 2014. 14(16): p. 3064-73.

34. David, F., et al., Viability and membrane potential analysis of Bacillus megaterium cells by impedance flow cytometry. Biotechnol Bioeng, 2012. 109(2): p. 483-92.

35. Mernier, G., et al., Cell viability assessment by flow cytometry using yeast as cell model. Sensors and Actuators B-Chemical, 2011. 154(2): p. 160-163. 
36. Shaker, M., et al., An impedance-based flow microcytometer for single cell morphology discrimination. Lab Chip, 2014. 14(14): p. 2548-55.

37. Song, $\mathrm{H}_{\text {., }}$ et al., A microfluidic impedance flow cytometer for identification of differentiation state of stem cells. Lab Chip, 2013. 13(12): p. 2300-10.

38. Du, E., et al., Electric impedance microflow cytometry for characterization of cell disease states. Lab Chip, 2013. 13(19): p. 3903-9.

39. Kuttel, C., et al., Label-free detection of Babesia bovis infected red blood cells using impedance spectroscopy on a microfabricated flow cytometer. Acta Trop, 2007. 102(1): p. 63-8.

40. Zheng, Y., et al., High-throughput biophysical measurement of human red blood cells. Lab Chip, 2012. 12(14): p. 2560-7.

41. Han, X., et al., Microfluidic lysis of human blood for leukocyte analysis using single cell impedance cytometry. Anal Chem, 2012. 84(2): p. 1070-5.

42. Evander, M., et al., Microfluidic impedance cytometer for platelet analysis. Lab Chip, 2013. 13(4): p. 722-9.

43. Bernabini, C., D. Holmes, and H. Morgan, Micro-impedance cytometry for detection and analysis of micron-sized particles and bacteria. Lab Chip, 2011. 11(3): p. 407-12.

44. Haandbaek, N., et al., Resonance-enhanced microfluidic impedance cytometer for detection of single bacteria. Lab Chip, 2014. 14(17): p. 331324.

45. Mernier, G., et al., Multiple-frequency impedance measurements in continuous flow for automated evaluation of yeast cell lysis. Sensor Actuat B-Chem, 2012. 170: p. 2-6.

46. Benazzi, G., et al., Discrimination and analysis of phytoplankton using a microfluidic cytometer. IET Nanobiotechnol, 2007. 1(6): p. 94-101.

47. Kirkegaard, J., et al., Study of Paclitaxel-Treated HeLa Cells by Differential Electrical Impedance Flow Cytometry. Biosensors, 2014. 4(3): p. 257-72.

48. Kang, G., et al., Differentiation Between Normal and Cancerous Cells at the Single Cell Level Using 3-D Electrode Electrical Impedance Spectroscopy. IEEE Sens J, 2012. 12(5): p. 1084-9.

49. Zhao, Y., et al., A microfluidic system for cell type classification based on cellular size-independent electrical properties. Lab Chip, 2013. 13(12): p. 2272-7.

50. Zhao, Y., et al., Tumor cell characterization and classification based on cellular specific membrane capacitance and cytoplasm conductivity. Biosens Bioelectron, 2014. 57: p. 245-53.

51. Watkins, N.N., et al., Microfluidic CD4+ and CD8+ Tlymphocyte counters for point-of-care HIV diagnostics using whole blood. Sci Transl Med, 2013. 5(214): p. 214ra170. 
52. Holmes, D. and H. Morgan, Single cell impedance cytometry for identification and counting of CD4 T-cells in human blood using impedance labels. Anal Chem, 2010. 82(4): p. 1455-61.

53. Segerink, L.I., et al., On-chip determination of spermatozoa concentration using electrical impedance measurements. Lab Chip, 2010. 10(8): p. 101824.

54. Myers, F.B., et al., Label-free electrophysiological cytometry for stem cellderived cardiomyocyte clusters. Lab Chip, 2013. 13(2): p. 220-8.

55. Gawad, S., L. Schild, and P.H. Renaud, Micromachined impedance spectroscopy flow cytometer for cell analysis and particle sizing. Lab Chip, 2001. 1(1): p. 76-82.

56. van Berkel, C., et al., Integrated systems for rapid point of care (PoC) blood cell analysis. Lab Chip, 2011. 11(7): p. 1249-55.

57. Barat, D., et al., Simultaneous high speed optical and impedance analysis of single particles with a microfluidic cytometer. Lab Chip, 2012. 12(1): p. 11826.

58. Zheng, Y., et al., Microfluidic characterization of specific membrane capacitance and cytoplasm conductivity of single cells. Biosens Bioelectron, 2013. 42: p. 496-502.

59. Pierzchalski, A., et al. Label-free single cell analysis with a chip-based impedance flow cytometer. Proc. of SPIE. 2010. 7568: p. 75681B-1-11.

60. Gou, H.L., et al., Label-free electrical discrimination of cells at normal, apoptotic and necrotic status with a microfluidic device. J Chromatogr A, 2011. 1218(33): p. 5725-9.

61. Crocetti, S., et al., Impedance flow cytometry gauges proliferative capacity by detecting TRPC1 expression. Cytom Part A, 2014. 85(6): p. 525-36.

62. Han, K.H., A. Han, and A.B. Frazier, Microsystems for isolation and electrophysiological analysis of breast cancer cells from blood. Biosens Bioelectron, 2006. 21(10): p. 1907-14.

63. Han, A., L. Yang, and A.B. Frazier, Quantification of the heterogeneity in breast cancer cell lines using whole-cell impedance spectroscopy. Clin Cancer Res, 2007. 13(1): p. 139-43.

64. Cho, Y., et al., Whole-Cell Impedance Analysis for Highly and Poorly Metastatic Cancer Cells. Jf Microelectromech S, 2009. 18(4): p. 808-17.

65. Jang, L.S. and M.H. Wang, Microfluidic device for cell capture and impedance measurement. Biomed Microdevices, 2007. 9(5): p. 737-43.

66. Mondal, D., et al., Microtrap electrode devices for single cell trapping and impedance measurement. Biomed Microdevices, 2012. 14(5): p. 955-64.

67. Tien Anh, N., I.Y. Tsung, and G. Urban. A cell impedance sensor chip for cancer cells detection with single cell resolution. in SENSORS, IEEE, 2013. 
68. Nguyen, T.A., et al., Microfluidic chip with integrated electrical cellimpedance sensing for monitoring single cancer cell migration in threedimensional matrixes. Anal Chem, 2013. 85(22): p. 11068-76.

69. Lan, K.C. and L.S. Jang, Integration of single-cell trapping and impedance measurement utilizing microwell electrodes. Biosens Bioelectron, 2011. 26(5): p. 2025-31.

70. Jang, L.S., P.H. Huang, and K.C. Lan, Single-cell trapping utilizing negative dielectrophoretic quadrupole and microwell electrodes. Biosens Bioelectron, 2009. 24(12): p. 3637-44.

71. Hong, J.L., K.C. Lan, and L.S. Jang, Electrical characteristics analysis of various cancer cells using a microfluidic device based on single-cell impedance measurement. Sensor Actuat B-Chem, 2012. 173: p. 927-34.

72. Chen, N.C., et al., Single-cell trapping and impedance measurement utilizing dielectrophoresis in a parallel-plate microfluidic device. Sensor Actuat BChem, 2014. 190(0): p. 570-7.

73. Malleo, D., et al., Continuous differential impedance spectroscopy of single cells. Microfluid Nanofluidic, 2010. 9(2-3): p. 191-98.

74. Cho, S. and $\mathrm{H}$. Thielecke, Micro hole-based cell chip with impedance spectroscopy. Biosens Bioelectron, 2007. 22(8): p. 1764-8.

75. Kurz, C.M., et al., Chip-based impedance measurement on single cells for monitoring sub-toxic effects on cell membranes. Biosens Bioelectron, 2011. 26(8): p. 3405-12.

76. Han, A. and A.B. Frazier, lon channel characterization using single cell impedance spectroscopy. Lab Chip, 2006. 6(11): p. 1412-4.

77. Asphahani, F., et al., Single-cell bioelectrical impedance platform for monitoring cellular response to drug treatment. Phys Biol, 2011. 8(1): p. 015006-1-23. 


\section{Microfluidic single sperm entrapment and analysis}

Selection of healthy sperm cells is of crucial important for the success rate of assisted reproduction technologies such as in-vitro fertilization and intracytoplasmic sperm injection. Although sperm selection for ART procedures is predominantly based on sperm motility, successful fertilization is not predicted by good motility alone. For example, sperm characteristics such as the acrosome state and DNA integrity have shown significant impact on ART outcome, Although fertilization can be achieved with a single spermatozoon of high quality, current quality assessments are population-based and do not allow investigation of multiple sperm characteristics on a single spermatozoon simultaneously. In order to study sperm cells on the single cell level, we designed and characterized a PDMS microfluidic platform that allows single sperm entrapment.

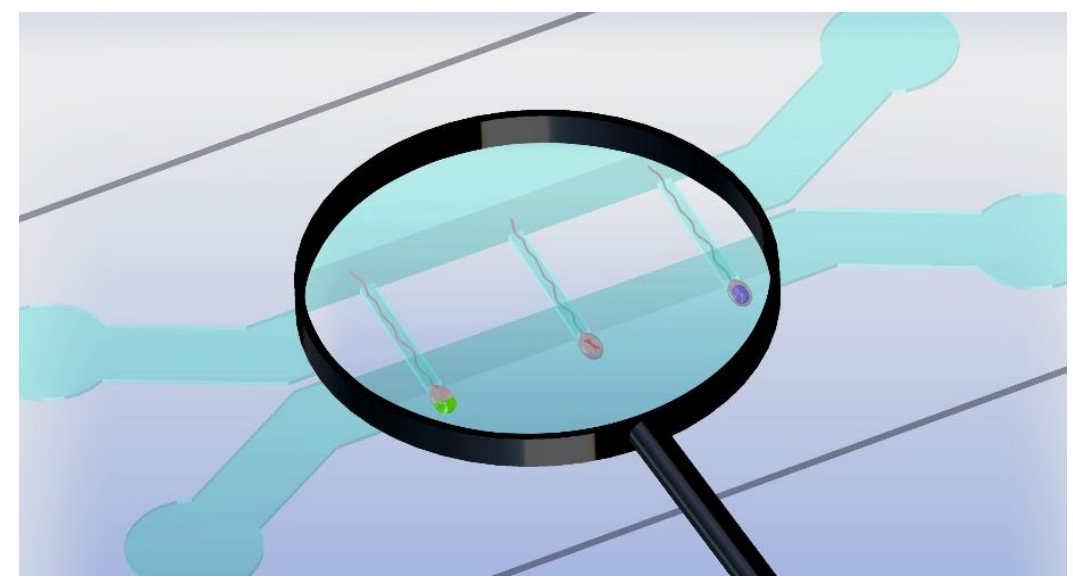

Graphical illustration of the single sperm trapping device (Lab on a chip inside back cover, 2015, 15)

Adapted from: de Wagenaar, B., Berendsen, J.T.W., Bomer, J.G., Olthuis, W., van den Berg, A., Segerink, L.I., Microfluidic single sperm entrapment and analysis. Lab Chip, 2015. 15(5): p.1294-301. 


\subsection{Introduction}

Nowadays, ART are commonly used to achieve pregnancy. Examples of ART procedures are intrauterine insemination (IUI), in-vitro fertilization (IVF) and intracytoplasmic sperm injection (ICSI) [1]. In order to ensure the highest probability of fertilization, sperm selection techniques such as sperm washing, sperm swim-up and density gradient centrifugation are used to obtain the most motile and viable sperm before performing ART procedures [2]. However, these selection techniques do not target all intrinsic sperm characteristics which have impact on ART outcome $[3,4]$. Advanced selection techniques focus on additional sperm characteristics such as DNA integrity, apoptosis, membrane maturation and ultramorphology $[3,5]$. Ideally, ART procedures are performed using viable, morphologically normal sperm cells with a high DNA integrity and intact plasma and acrosomal membranes. Especially, ICSI treatments are dependent on accurate selection, since only one spermatozoon is used during each procedure. However, most selection procedures and sperm quality related studies rely on populationbased approaches, which are not applicable (yet) on the single cell level. The lack of comprehensive single cell information could explain why sperm selection based on characteristics, which showed correlation with fertilization potential in population-based studies, fails to achieve clinical relevance in ART procedures. As an example, the significance of DNA integrity on reproductive outcome is both supported [6, 7] and contradicted [8, 9].

A potential way to manipulate and study sperm cells on the single cell level is the use of microfluidic technology. In the last decennium, a growing number of reports showed the potential to perform sperm handling and selection in microfluidic systems [10]. These systems have shown important advantages over traditional selection techniques such the potential to work with small sample volumes, short processing times and the ability to manipulate single cells. Various reports show the ability to separate motile sperm cells by their ability to swim through microchannels [11,12], to cross laminar flows $[13,14]$ or to swim against fluid flows [15-17]. These microfluidic systems are able to select for viable and motile sperm cells, avoiding induced cell damage inflicted by routine ART procedures. However, these platforms do not allow sperm manipulation and analysis on the single cell level.

Few reports focus on the microfluidic manipulation and analysis of single sperm cells. In a report of Fuhr et al. [18] individual sperm cells were entrapped using dielectrophoresis, allowing the investigation of the sperm motility. Ohta et al. [19] 
used laser tweezers to entrap sperm cells based on membrane integrity. In both approaches, complicated methods were used to trap the sperm cells, which are potentially invasive, which limits their use for clinical applications. Furthermore, no extensive analysis of the sperm cells quality was reported. In previous work in our group we trapped single sperm cells on fibronectin spots, allowing automatic analysis of the sperm's motility for ICSI purposes [20]. However, this platform is less suitable for fluorescent staining procedures.

We report the design of a simple PDMS microfluidic platform in which sperm cells can be entrapped non-invasively by hydrodynamics. Sperm characteristics such as the cell viability, the acrosome state and chromosomal content were studied on individual entrapped sperm cells. This platform allows (non-invasive) analysis on the single cell level and has the potential to be a versatile tool for selection applications or fundamental studies on sperm cells.

\subsection{Materials \& methods}

\subsubsection{Microfluidic setup \& chip fabrication}

The microfluidic chip consists of two main channels, which are interconnected by small side channels (Fig. 4-1). The chip was designed using CleWin software (version 4.0.1). Master molds for PDMS fabrication were produced by standard photolithography. In short, two layers of SU-8 (Microchem) were spun and developed on 4" silicon wafers. The first and second layer contains the design features of the side channels and main channels, respectively. Three different SU8 molds were fabricated, varying the first layer thickness between 1, 1.5 and $2 \mu \mathrm{m}$ (fabrication results $0.96,1.49$ and $1.98 \mu \mathrm{m}$ respectively, Bruker Dektak 8 ). The total thickness of both layers combined was $20 \mu \mathrm{m}$.

Chips were fabricated using PDMS (Sylgard 184, Dow Corning) in a 1:10 v/v ratio of base versus curing agent. PDMS was poured onto a silicon wafer, degassed and cured at $60{ }^{\circ} \mathrm{C}$ overnight. After curing, microfluidic in- and outlets were punched using Harris Uni-Core punchers (tip ID 1.0 and $3.0 \mathrm{~mm}$, Ted Pella Inc.). The chips were bonded to glass microscope slides using oxygen plasma (Harrick PDC-001). Finally, the chips were baked at $60^{\circ} \mathrm{C}$ for at least $30 \mathrm{~min}$ before use.

\subsubsection{PLL-g-PEG surface coating}

PDMS and glass surfaces were coated with poly(L-lysine)-grafted-poly(ethylene glycol) (PLL(20)-g[3.5]-PEG(2), SuSoS) to prevent cell adhesion during cell trapping 
experiments. PLL-g-PEG was rinsed through the PDMS microchannels at a concentration of $100 \mu \mathrm{g} \cdot \mathrm{mL}^{-1}$ in DI water for at least $15 \mathrm{~min}$.

\subsubsection{Sample preparation}

Fresh boar semen was obtained from a local artificial insemination centre ("KI Twenthe", Fleringen, The Netherlands) at a concentration of $20 \cdot 10^{6}$ cells $\mathrm{mL}^{-1}$. The samples were diluted with Beltsville Thawing Solution (BTS, Solusem, Aim Worldwides) to a concentration of $2 \cdot 10^{6}$ cells $\mathrm{mL}^{-1}$ before trapping experiments.

\subsubsection{Hydrodynamic cell trapping}

Two $100 \mu \mathrm{L}$ syringes (Hamilton gastight, 1710N, Reno, NV, USA) were connected to the $1 \mathrm{~mm}$ chip outlets using fused silica capillaries (Polymicro technologies, ID 100 $\mu \mathrm{m}$, OD $360 \mu \mathrm{m}$ ) and Tygon tubing (ND 100-80, ID $250 \mu \mathrm{m}$, OD $760 \mu \mathrm{m}$, Saint-Gobain Performance Plastics). Two constant fluid flows were established by drawing liquid from the $3 \mathrm{~mm}$ inlet reservoirs to the outlets using a syringe pump (neMESYS, Cetoni $\mathrm{GmbH}$ ). First, channels were rinsed with a PLL-g-PEG coating solution for 15 $\mathrm{min}$ at flow rates of $0.25 \mu \mathrm{L} \mathrm{min}{ }^{-1}\left( \pm 208.3 \mu \mathrm{m} \cdot \mathrm{s}^{-1}\right)$. Subsequently, BTS was flushed through the system for $15 \mathrm{~min}$ at equal flow rates to remove remaining coating solution. After introducing the sperm sample to the top channel, the flow rates of the top and bottom channel were adjusted to 0.025 and $2.5 \mu \mathrm{L} \cdot \mathrm{min}^{-1}$ respectively. Due to the resulting pressure difference, cells were trapped within the side channels. Sperm trapping experiments were performed within 1 to 2 minutes. After trapping, the sperm solution was replaced by BTS and a mild pressure difference was retained using top and bottom channel flow rates of 0.025 and $0.5 \mu \mathrm{L} \cdot \mathrm{min}^{-1}$ to prevent sperm cells to escape from the traps.

\subsubsection{Viability staining}

Sperm viability staining was performed (on-chip) using SYTO 9 (ex/em 485/498, Invitrogen) and propidium iodide (PI, ex/em 535/617, Life Technologies) nucleic stains. Sperm cells were pre-stained using a BTS solution with $3.34 \mu \mathrm{M}$ SYTO 9 for $5 \mathrm{~min}$ before trapping in the microfluidic device. After trapping, a $10 \mu \mathrm{g} \cdot \mathrm{mL}^{-1} \mathrm{PI}$ in BTS solution was rinsed through the channel at a flow rate of $0.025 \mu \mathrm{L} \cdot \mathrm{min}^{-1}$ at room temperature. Viability of entrapped sperm cells and non-trapped, control sperm cells (pipetted on a glass slides) was investigated at 15 and 45 min after cell trapping. Each control experiment was performed right after a cell trapping experiment using the same sperm solution. Fresh sperm solutions were prepared before each viability experiment. A Nikon TE2000-U microscope equipped with a 
10x phase contrast objective, DS-RI1 camera, Nikon Intensilight C-HGFIE and FITC \& PI filter cubes (49011 \& 41005, Chroma technologies) were used.

\subsubsection{Fluorescent in situ hybridization}

The $X$ and $Y$-chromosomes of sperm cells were stained with florescent in-situ hybridization (FISH) using porcine $\mathrm{X}$ - and $\mathrm{Y}$-chromosome specific probes in hybridization buffer (Idetect, IDPF1078 ex/em 493/521 and IDPR1066 ex/em $548 / 573$, ID Labs). The off and on-chip staining procedures are described separately.

\section{Off-chip staining}

FISH staining of sperm cells was based on detailed protocols described elsewhere. $[21,22]$ In short, $1 \times 10^{6}$ sperm cells $\mathrm{mL}^{-1}$ were incubated in a $0.075 \mathrm{M} \mathrm{KCl}$ (Sigma Aldrich) hypotonic solution at $37^{\circ} \mathrm{C}$ for $30 \mathrm{~min}$ and fixated using Carnoy's fixative ( $3: 1 \mathrm{v} / \mathrm{v}$ methanol:acetic acid; Sigma Aldrich) afterwards. After washing the fixated cells with $2 x$ saline-sodium citrate (SSC; Fisher Bioreagents), the cells were incubated in a freshly prepared dithiothreitol (DTT) solution $(5 \mathrm{mM} \mathrm{1,4-}$ dithiothreitol, 1\% v/v Triton X-100 and $50 \mathrm{mM}$ 2-amino-2-(hydroxymethyl)-1,3propanediol in DI water; purchased at Acros organics, Sigma Aldrich and Acros organics, respectively) at $37{ }^{\circ} \mathrm{C}$ for $15 \mathrm{~min}$ to decondense the chromatin. After incubation, the cells were washed twice in $2 \times$ SSC for $3 \mathrm{~min}$ and in ethanol $(70 \%$, $85 \%$ and $100 \%$ ) for 1 min each. Subsequently, a hybridization mixture of $X$ and $Y$ chromosome specific DNA probes and hybridization buffer ( $50 \% \mathrm{v} / \mathrm{v})$ was added and co-denatured with the chromosomal DNA at $73^{\circ} \mathrm{C}$ for $3 \mathrm{~min}$. Hybridization was performed in polycarbonate hybridization chambers (Corning Inc.) at $37{ }^{\circ} \mathrm{C}$ overnight. After incubation, the cells were washed using a $0.4 \mathrm{x} \mathrm{SSC}$ with $0.3 \% \mathrm{v} / \mathrm{v}$ Tween-20 (Acros organics) washing solution at $73^{\circ} \mathrm{C}$ for $3 \mathrm{~min}$. Finally, cells were washed with DI water and mounted with Vectashield (Vector Laboratories, Inc.). Fluorescent visualization was performed using an EVOS FL cell imaging system (Life Technologies) equipped with a 40x objective and GFP and RFP filter cubes.

\section{On-chip staining}

On-chip FISH staining was performed by adapting the previous described FISH protocol. All reagents were rinsed through the channels at a flow rate of 0.05 $\mu \mathrm{L} \cdot \mathrm{min}^{-1}$ unless mentioned otherwise. First, cell trapping was performed as described in section 4.2.4. After trapping, a $37^{\circ} \mathrm{C}$ solution of $0.075 \mathrm{M} \mathrm{KCl}$ was rinsed through both channels for $30 \mathrm{~min}$. A microscope hot plate was used to retain the temperature at $37{ }^{\circ} \mathrm{C}$. After hypotonic treatment, Carnoy's fixative was rinsed 
through the channels for $10 \mathrm{~min}$ to fix the sperm cells. Subsequently, the cells were washed with $2 \times$ SSC for 2 min after which they were treated with DTT solution at $37^{\circ} \mathrm{C}$ for $20 \mathrm{~min}$. Afterwards, $2 \times$ SSC and ethanol washes were performed for $2 \mathrm{~min}$ each.

After decondensation, the hybridization mix was added to the cell-containing top channel, while adding hybridization buffer to the bottom channel. After sealing the microfluidic inlets with parafilm, the chips were placed on a hot plate at $73^{\circ} \mathrm{C}$ for 3 min to denature the probes and DNA. Afterwards, they were placed in an incubator, allowing hybridization at static flow conditions at $37^{\circ} \mathrm{C}$ overnight.

After hybridization, the parafilm was removed and $0.4 \times$ SSC washing solution was rinsed through the channels at a flow rate of $0.25 \mu \mathrm{L} \cdot \mathrm{min}^{-1}$ for $1 \mathrm{~min}$. The flow was stopped and the chip was placed on a hotplate at $73^{\circ} \mathrm{C}$ for $2.5 \mathrm{~min}$. Afterwards the flow was adjusted to $0.1 \mu \mathrm{L} \cdot \mathrm{min}^{-1}$ and $2 \mathrm{x} \mathrm{SSC}$, DI water and Vectashield were rinsed through the channels for 1 min each. Finally, the chips were completed by removing the tubing and by covering the in- and outlets with a coverslip. Finished slides were stored at $4{ }^{\circ} \mathrm{C}$ before evaluation using the EVOS FL microscope.

\subsubsection{Acrosome staining}

Acrosome staining was performed using a fluorescein-conjugated pisum sativum agglutinin (FITC-PSA, ex/em 422/544, Vector Laboratories, Inc.) and LysoTracker blue (DND-22, ex/em 373/422, Life Technologies) double stain to distinguish between acrosome-damaged and acrosome intact sperm cells, respectively. After trapping, sperm cells were incubated in BTS solution with $25 \mu \mathrm{g} \cdot \mathrm{mL}^{-1}$ FITC-PSA and $2.5 \mu \mathrm{M}$ LysoTracker blue for $1 \mathrm{~h}$ at $37{ }^{\circ} \mathrm{C}$ with static flow conditions. After incubation, acrosome staining was visualized using an EVOS FL microscope equipped with a 100x oil immersion objective and DAPI, GFP and RFP filter cubes.

\subsubsection{SEM imaging}

Scanning electron microscopy (SEM) images were created using a JEOL JSM 5610 after sputtering a thin layer of chromium on the PDMS chips using a chromium sputter coater (Emitech). All images were recorded at an acceleration potential of $10 \mathrm{kV}$. 


\subsection{Results \& discussion}

\subsubsection{Device design \& simulation}

An important requirement of the microfluidic device is the ability to entrap sperm cells individually. The PDMS chip design (Fig. 4-1) consists of two main channels, which are interconnected by twenty side channels. These side channels will act as cell traps when a pressure gradient is induced between the main channels. A channel width of $2 \mu \mathrm{m}$ allows fluid flow between the two main channels, but will prevent sperm cells (head length of $9 \mu \mathrm{m}$, width of $4.5 \mu \mathrm{m}$ and a thickness of 0.5 $\mu \mathrm{m}[23])$ to cross to the other main channel.

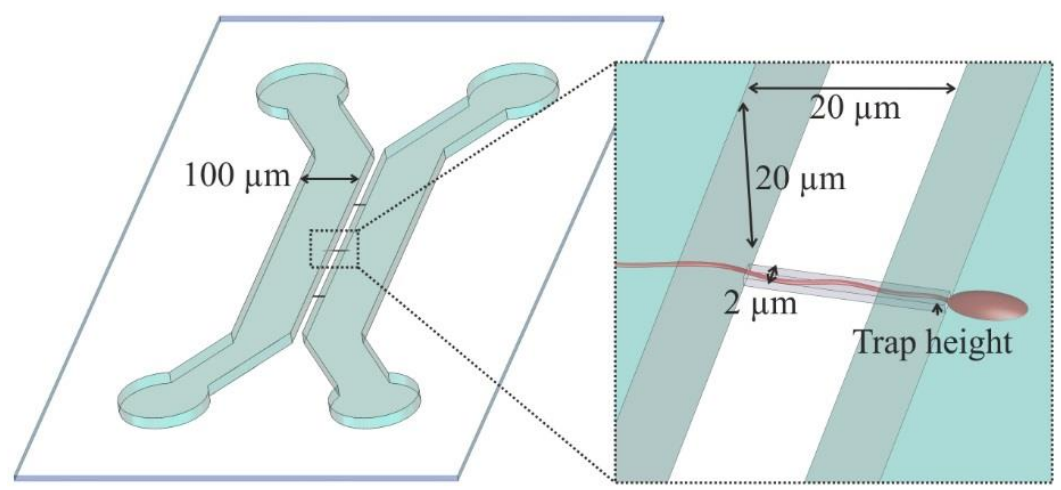

Fig. 4-1: Illustration and imaging of the microfluidic platform. The PDMS chip design, when bonded to a glass substrate, consists of two main channels (width $100 \mu \mathrm{m}$, height $20 \mu \mathrm{m}$ ) and twenty side channels (width $2 \mu \mathrm{m}$, length $20 \mu \mathrm{m}$ ), which connect the main channels. The height of these side channels, i.e. trap height, is $1,1.5$ or $2 \mu \mathrm{m}$.

The height of the side channels, i.e. trap height, was varied $(1,1.5$ or $2 \mu \mathrm{m})$ to optimize single cell trapping. The spacing between the side channels is $150 \mu \mathrm{m}$. The topography of a fabricated PDMS chip with a trap height of $1 \mu \mathrm{m}$ was visualized by scanning electron microscopy (SEM). SEM imaging showed smooth PDMS walls and well defined cell traps (Fig. 4-2). To study the fluid dynamics in this microfluidic chip, a COMSOL Multiphysics model (version 4.4) was constructed (Fig. 4-3). This model describes a laminar flow of incompressible fluid (water) through the microfluidic device under no slip conditions, applying zero pressure at the main channel inlets and fluid outflow velocities at the channel outlets. 
Due to the difference in outflow velocity $\left(0.025\right.$ versus $\left.2.5 \mu \mathrm{L} \mathrm{min}{ }^{-1}\right)$, a pressure gradient is formed over the side channels (Fig. 4-3A). As a result, fluid flow is induced within these channels, which is illustrated by arrows representing the fluid flow (Fig. 4-3B). In practice, this fluid flow will drag and entrap passing sperm cells within the side channels.
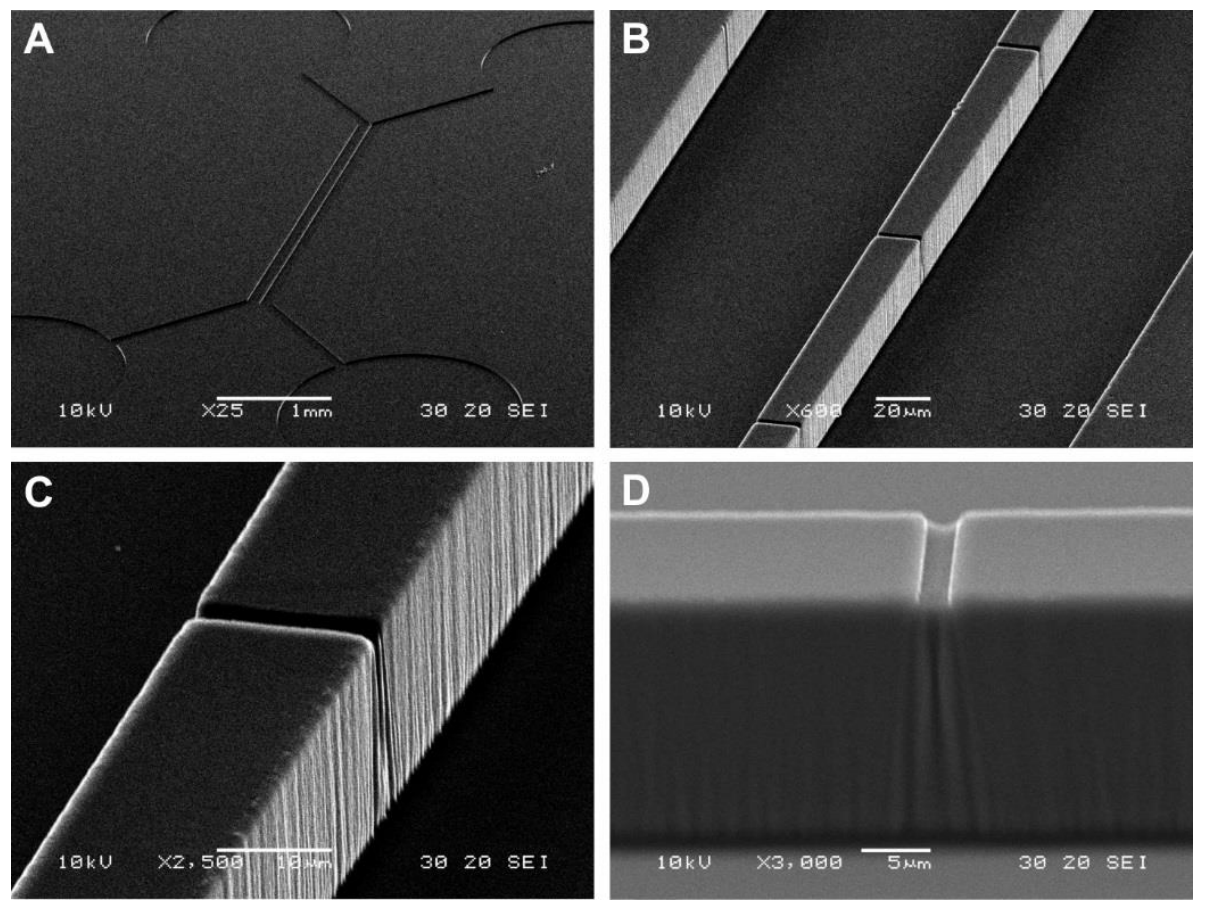

Fig. 4-2: Scanning electron microscopy images of the PDMS chip using A) $25 x$, B) $600 x$, C) $2.500 x$ and D) $3.000 x$ magnification. All images were recorded using an acceleration potential of $10 \mathrm{kV}$.

The pressure difference over a cell trap depends upon the distance between the trap and the main channel outlet. This difference is observed to linearly decrease with increasing distance, i.e. increasing trap number (Fig. 4-4). According to this simulation, traps close to the channel outlet will likely be the first to entrap sperm cells in trapping experiments. In the scenario in which trapped sperm cells completely block the fluid flow through the side channels, the fluid flow through the neighbouring, unfilled side channels will gradually increase upon cell trapping. 

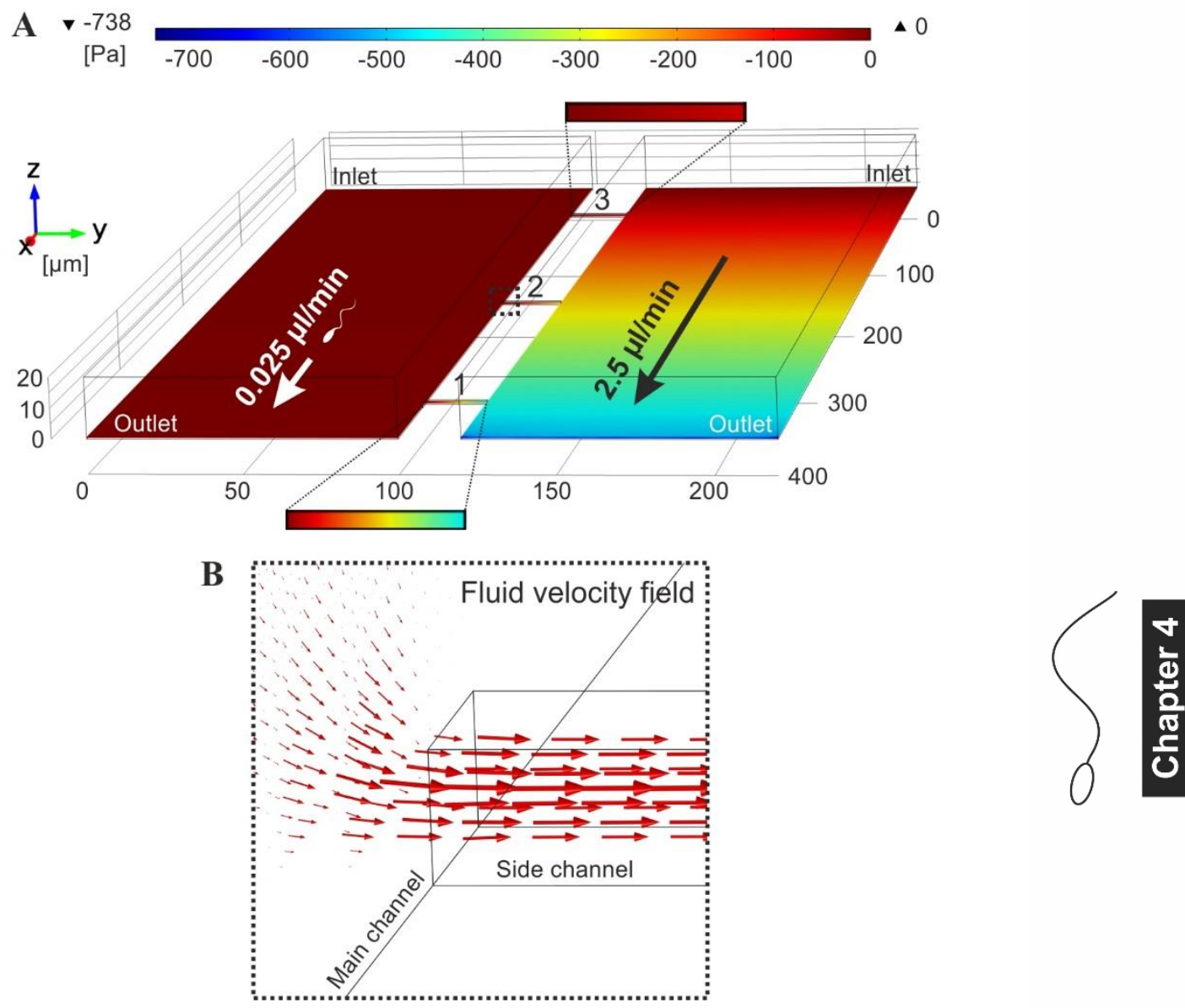

Fig. 4-3: COMSOL Multiphysics (version 4.4) simulation of the A) fluid flow within the microfluidic trapping device, showing the first three cell traps. A fluid flow of $0.025 \mu \mathrm{L} \cdot \mathrm{min}^{-1}$ and $2.5 \mu \mathrm{L} \cdot \mathrm{min}^{-1}$ is applied to the left and right main channel, respectively. Due to the difference in flow rates, a pressured gradient is created between the main channels. This is illustrated by the pressure distribution within the device at a height of $0.5 \mu \mathrm{m}$, which is half the height of the side channels. As a result of this B) pressure difference, a fluid flow is induced through these side channels, which is illustrated by arrows representing the direction and magnitude of the fluid flow.

Consequently, the distance between the cell traps and channel outlet will not influence their cell trapping capability. However, a trapped spermatozoon will never completely block the fluid flow through a side channel since its thickness is 


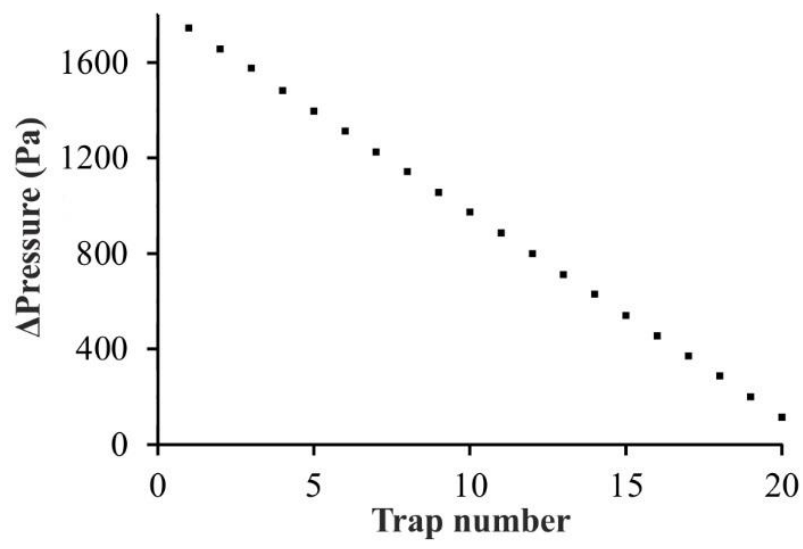

Fig. 4-4: The pressure difference over a cell trap (between side channel inlet and outlet, total length $20 \mu \mathrm{m}$ ) linearly decreased with increasing trap number at a z-height of $0.5 \mu \mathrm{m}$.

only half the size of the channel height. This leakage flow will affect the trapping capability of neighbouring cell traps. Therefore, we expect to observe a decrease in trap occupancy with increasing distance between trap and channel outlet.

\subsubsection{Single cell entrapment}

Sperm trapping was performed using side channels with a height of 1, 1.5 and $2 \mu \mathrm{m}$ to find the optimal trap height for single sperm entrapment. Cell trapping was performed within 1 to 2 minutes after applying the pressure difference. Experiments showed effective single cell trapping using $1 \mu \mathrm{m}$ high traps (Fig. 4-5A). Entrapped sperm cells were orientated in a head-first or tail-first orientation with respect to the cell trap, in which the tail-first position proved to be the most stable. The $1.5 \mu \mathrm{m}$ traps showed single sperm entrapment although occasionally two sperm cells were caught (Fig. 4-5B). Increasing the height to $2 \mu \mathrm{m}$ resulted in an increase of multiple cell trapping (Fig. 4-5C), catching up to 5 sperm cells per spot. Furthermore, an increased number of sperm cells were caught with their heads in a perpendicular direction with respect to the cell trap, i.e. aligning the flat side of their heads with the PDMS wall. After each trapping experiment, the percentage of empty traps, traps containing a single cell or traps containing multiple cells on each chip was recorded independently of trap number. This percentage was clearly influenced by the cell trap height (Fig. 4-5D, $n=3$ ). The highest ratio of single versus multiple cell trapping was obtained using chips with $1 \mu \mathrm{m}$ high traps. This ratio was observed to decrease with increasing trap height. 


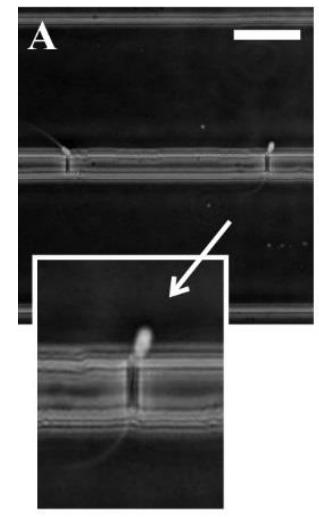

D 80
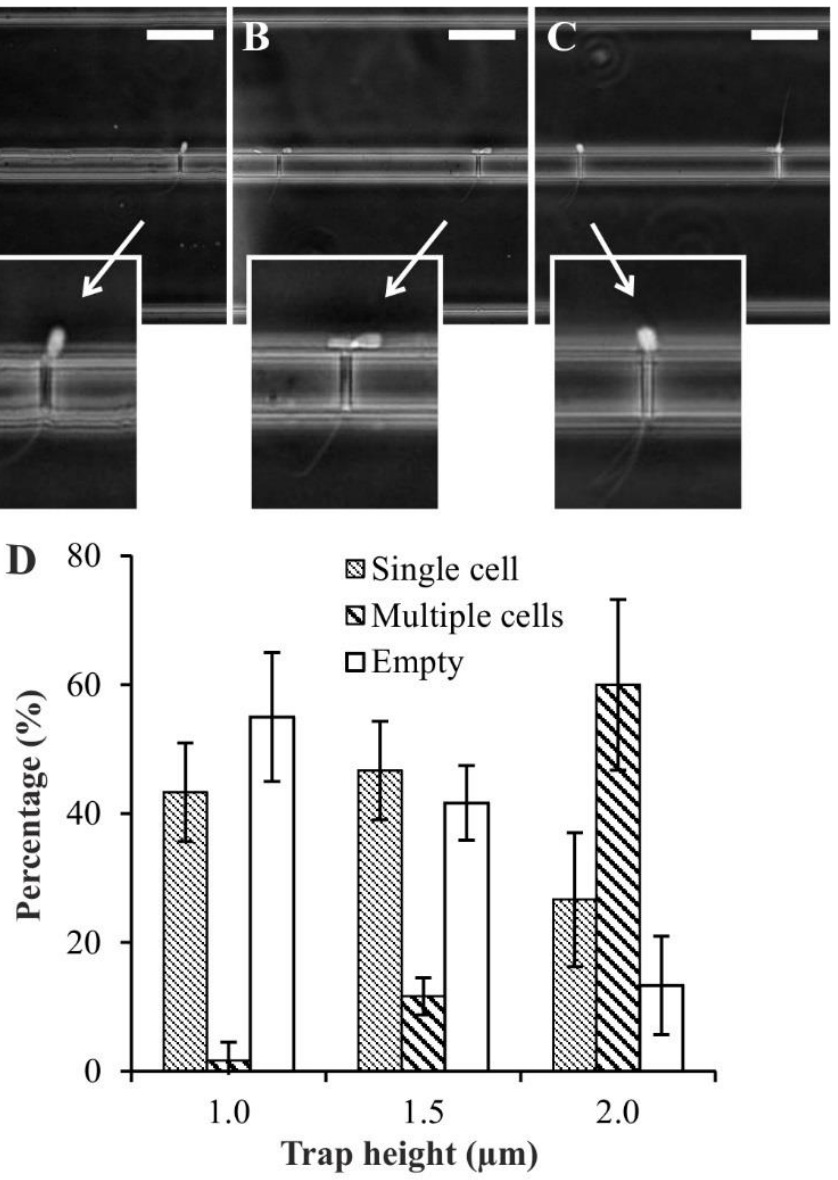

Fig. 4-5: Entrapment of sperm cells in $2 \mu \mathrm{m}$ wide PDMS traps with a height of A) $1 \mu \mathrm{m}, \mathrm{B}) 1.5 \mu \mathrm{m}$ and C) $2 \mu \mathrm{m}$. D) The percentage of traps filled with no cells, a single cell or multiple cells was recorded and plotted versus trap height. The percentage of single cell trapping was highest for chips with a trapping height of $1.5 \mu \mathrm{m}$. The highest ratio of single versus multiple cell trapping was obtained using chips with a trap height of $1 \mu \mathrm{m}$. (experiments per trap height $\mathrm{n}=3$, error bars $=1 \mathrm{x}$ standard deviation, all scale bars are $50 \mu \mathrm{m}$ ).

Unfortunately, the highest amount of empty traps was observed using a trap height of $1 \mu \mathrm{m}$. Despite this drawback, a trapping height of $1 \mu \mathrm{m}$ was considered most suitable for single sperm trapping and analysis. Now, chips with $1 \mu \mathrm{m}$ high cell traps were used to investigate the trapping efficiency (Fig. 4-6). In total, 20 trapping experiments were performed as described in paragraph 4.2.4. 


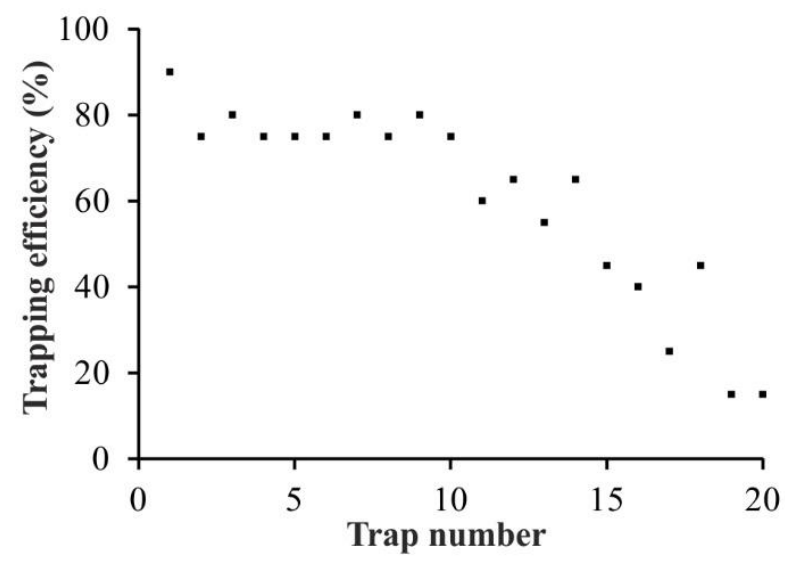

Fig. 4-6: The cell trapping efficiency as a function of trap number. A clear decrease in efficiency is observed between traps 10 and $20(n=20)$.

After each experiment, the trap occupancy was recorded for every cell trap in order to calculate the trap efficiency. This efficiency is expressed as the percentage of successful cell entrapment over all experiments. For example, trap 1 showed $90 \%$ efficiency, i.e. in $90 \%$ of all experiments a spermatozoon was entrapped. The data does not show a clear trend; both linear as polynomial trends do not show statistical significance (data not shown). However, the efficiency decreased with trap number, which is in good correspondence with our expectations from the simulation.

\subsubsection{Viability staining}

The hydrodynamic trapping procedure could inflict a harmful effect on the sperm cells. To investigate this effect, the viability was assessed by monitoring the plasma membrane integrity using SYTO 9 and PI nucleic acid stains at two different time points. Cell membrane permeable SYTO 9 stain (green excitation) is able to bind to the DNA of sperm cells with intact plasma membranes (i.e. viable sperm cells). However, PI dye (red excitation) is only able to bind to the DNA of sperm cells with damaged plasma membranes (i.e. non-viable sperm cells). As an example, three entrapped sperm cells showed intact plasma membranes after 15 min (Fig. 4-7A) and one deteriorated membrane after $45 \mathrm{~min}$ (Fig. 4-7B).The decrease in intensity after 45 min was caused by photobleaching of the green fluorophore. 

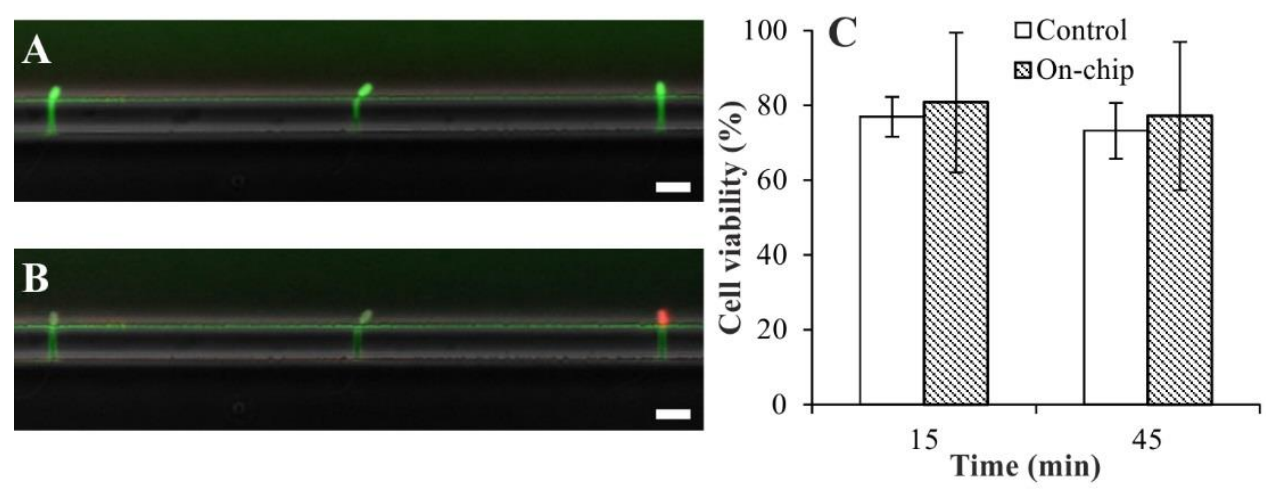

Fig. 4-7: Cell viability staining of trapped sperm cells using a SYTO 9 / PI solution. A) The viability was monitored at $15 \mathrm{~min}$ and B) $45 \mathrm{~min}$ after cell trapping. Viable sperm cells with intact membranes were visualized by a clear green fluorescent signal; deterioration of the plasma membrane was indicated by a red fluorescent signal. C) No difference in viability was observed between the control and on-chip group after 15 min and 45 min after trapping ( $n=7$, all scale bars are $20 \mu \mathrm{m})$.

The cell viability was assessed by ten separate viability experiments. The results of three experiments were excluded from the presented data. In two of these experiments, more than $50 \%$ of the initially trapped sperm cells at 15 min were lost after $45 \mathrm{~min}$. In the other experiment, the control sample showed a significantly lower cell viability compared to other control experiments. On average, cell counts were $122 \pm 30(n=7)$ for each control experiment and $15 \pm 2(n=7)$ for each onchip experiment. High cell viability was observed for both the control and on-chip group (Fig. 4-7C). The sperm viability of both groups remained constant over time.

No significant differences in viability are observed between both groups at both time points, indicating that the viability of the entrapped sperm cells was not compromised by the trapping procedure. Furthermore, the non-invasive nature of the trapping procedure was supported by observing motile sperm cells within the microfluidic traps after the trapping procedure (results hot shown).

All viability experiments were performed using PDMS devices in which the surface coating was performed using optimized procedures. Coating with old PLL-g-PEG solution (>1 week) or a prolonged duration between chip bonding and coating (>30 min) showed a negative effect on cell viability (data not shown). No clear 
explanation was found for this observation. Potentially, a reduced surface coverage played a role in the decrease in sperm viability. Literature on PLL-g-PEG coating of PDMS shows that the absorption of polymer on oxidized surfaces is higher compared to absorption on hydrophobic surfaces [24, 25]. Bad surface coverage could result in direct contact between the PDMS surface and sperm cells upon cell trapping. Although PDMS is biocompatible in general, the fragile nature of sperm cells could be compromised by leaching of absorbed trace elements [26] or hydrophobic materials such as PDMS uncrosslinked oligomers [27]. In short, a decreased PLL-g-PEG surface coverage due to its reduced absorption on hydrophobic PDMS surfaces could result in the increase of leaching of toxic components, resulting in a decrease of sperm viability.

\subsubsection{Fluorescent in situ hybridization}

FISH is a commonly used technique to investigate chromosome content and chromosomal anomalies of sperm cells, such as sperm aneuploidy [28]. The presence of chromosomal anomalies has shown correlation with male infertility [29] and ICSI outcome [30]. To test whether chromosome analysis can be performed on entrapped sperm cells in the current microfluidic device, a FISH procedure was designed on-chip. In this example, the sex chromosome content is investigated using $\mathrm{X}$ and $\mathrm{Y}$-chromosome specific DNA probes.
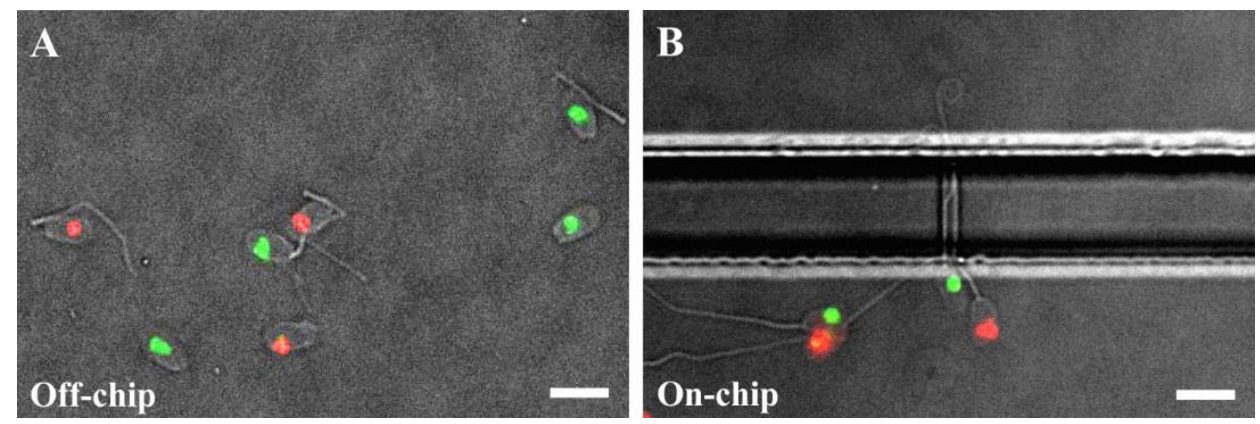

Fig. 4-8: A) Fluorescent in-situ hybridization of the $X$ and $Y$ chromosomes, yielding sperm cells with a green or red fluorescent signal, respectively. This staining protocol was performed B) on-chip to investigate the sex-chromosome content of entrapped sperm cells (all scale bars are $10 \mu \mathrm{m})$. 
2FISH control experiments showed successful hybridization of the two DNA probes on the $\mathrm{X}$ and $\mathrm{Y}$-chromosomes, indicated by green and red fluorescent signals respectively (Fig. 4-8A). The staining technique was performed on-chip, yielding entrapped sperm cells with stained $X$ - and Y-chromosomes (Fig. 4-8B). In this experiment, chips were used with a trap height of $2 \mu \mathrm{m}$ without PLL-g-PEG surface coating, which explains the number of multiple entrapped sperm cells. These results show the potential to perform FISH analysis on-chip, allowing the analysis of chromosomal anomalies of individually trapped sperm cells.

\subsubsection{Acrosome staining}

The acrosome reaction, which involves the activation of proteolytic enzymes to digest the zona pellucida, plays a crucial role in the fertilizing potential of sperm cells [31]. In vitro analysis using the ionophore-induced acrosome reaction (ARIC) test showed effective in predicting fertilization potential in IUI and IVF treatments [32].

Analysis of the acrosome state of entrapped sperm cells was performed using FITCPSA and LysoTracker blue, staining acrosome-reacted and acrosome-intact sperm cells respectively. This double acrosome staining was combined with $\mathrm{PI}$, allowing investigation of the integrity of the acrosomal and plasma membranes simultaneously. Bonding of PSA to exposed lectins of damaged acrosomal membranes resulted in a clear green fluorescence (Fig. 4-9ABC). Non-specific green fluorescence was caused by particles of non-dissolved PSA, which were trapped besides of the sperm cells. Sperm cells with intact acrosomes were identified by the absence of lectin-bound PSA and the presence of LysoTracker blue.

Absorption of the LysoTracker dye in the weak acidic environment $(\mathrm{pH} \approx 5)$ of an intact acrosome yielded a clear blue fluorescent signal (Fig. 4-9A). Despite of high background fluorescence due to absorbed fluorophore within the PDMS matrix, sperm cells with intact acrosomes could be distinguished on-chip (Fig. 4-9D). Potentially, this absorption can be reduced or prevented by coating the chips with a layer of parylene [33].

\subsubsection{Device implementation}

The potential of the presented microfluidic device to entrap individual sperm cells, allows the use of non-invasive analysis techniques which depend upon experiments on the long time-scale. Two promising techniques are Raman and impedance spectroscopy. 

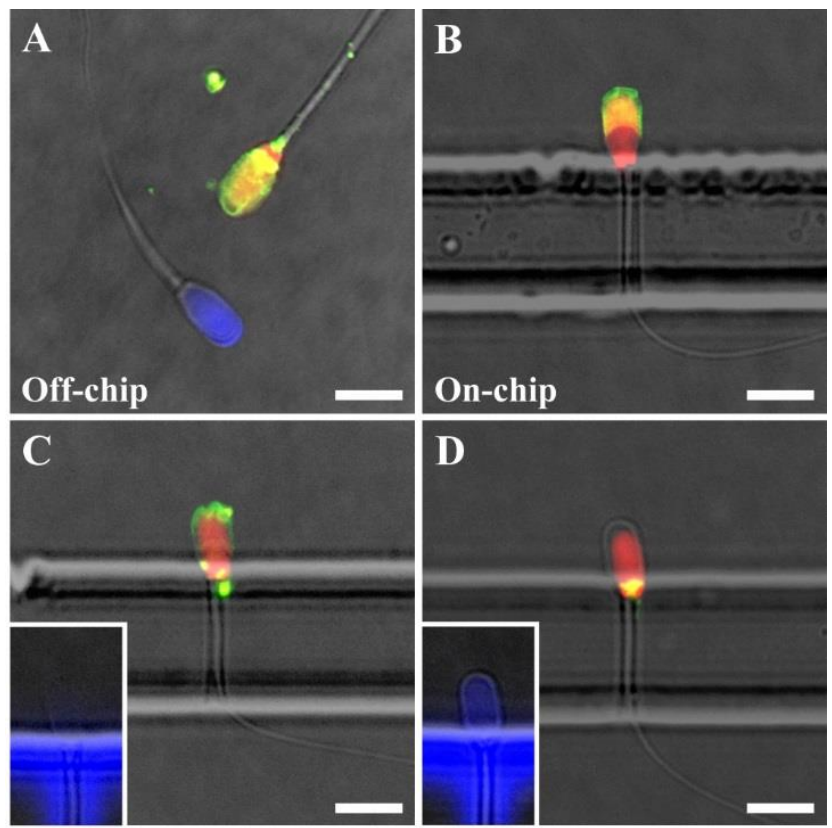

Fig. 4-9: Acrosome staining of sperm cells. A) Reacted and unreacted acrosomes were visualized using FITC-PSA and LysoTracker blue, respectively. PI was used to test the integrity of the plasma membrane. This staining procedure was used to evaluate the acrosome state of sperm cells trapped on-chip. On-chip staining results showed entrapped sperm cells with $B \& C$ ) reacted and D) intact acrosomes (all scale bars are $10 \mu \mathrm{m}$ ).

Although Raman spectroscopy was used to study sperm DNA damage and the acrosomal membrane [34,35], no studies have been conducted on viable sperm cells so far. On the contrary, numerous reports show the use of impedance spectroscopy to study single cells non-invasively using microfluidic platforms [36, 37]. Therefore, our future work will focus on the characterization of the sperm quality using impedance spectroscopy. The fluorescent staining procedures described in this report will be used for verification purposes.

Besides integrating the trapping device with a non-invasive analysis technique, the platform must allow the recovery of single sperm cells selectively. Possible solutions to recover single sperm cells are multiplexing the current design with additional channels or the use of small diameter needles as shown in previous work [20]. 


\subsection{Conclusions}

In this study, we designed and characterized a microfluidic platform for the entrapment and analysis of single sperm cells. Effective single cell entrapment was accomplished using a hydrodynamic trapping procedure using rectangular cell traps with a width of $2 \mu \mathrm{m}$ and a height of $1 \mu \mathrm{m}$. Single cell analysis was performed on entrapped sperm cells, studying the sperm viability, chromosome content and the acrosome state. In future, this platform can be exploited for the advanced analysis of single sperm cells. Furthermore, this platform can be integrated with noninvasive analysis methods, such as Raman and impedance spectroscopy, to utilize non-invasive analysis and selection for ICSI purposes.

\subsection{Acknowledgements}

Financial support from the NWO - Netherlands Organization of Scientific Research (Spinoza Grant A. van den Berg, Veni L.I. Segerink), the scientific support of N. Huisintveld, J.P. Frimat, C.C.L. Denoeud, I. van de Ven and S. Sukas and the technical support of P.M. ter Braak and J.B. Bomer are gratefully acknowledged. We also thank the "KI Twenthe" for the kind supply of boar semen samples.

\subsection{References}

1. Ferraretti, A.P., et al., Assisted reproductive technology in Europe, 2008: results generated from European registers by ESHRE dagger. Hum Reprod, 2012. 27(9): p. 2571-84.

2. Henkel, R.R. and W.B. Schill, Sperm preparation for ART. Reprod Biol Endocrinol, 2003. p. 1:108.

3. Said, T.M. and J.A. Land, Effects of advanced selection methods on sperm quality and ART outcome: a systematic review. Hum Reprod Update, 2011. 17(6): p. 719-33.

4. Yetunde, I. and M. Vasiliki, Effects of advanced selection methods on sperm quality and ART outcome. Minerva Ginecol, 2013. 65(5): p. 487-96.

5. Henkel, R., Sperm preparation: state-of-the-art--physiological aspects and application of advanced sperm preparation methods. Asian J Androl, 2012. 14(2): p. 260-9.

6. Benchaib, M., et al., Sperm DNA fragmentation decreases the pregnancy rate in an assisted reproductive technique. Hum Reprod, 2003. 18(5): p. 1023-8. 
7. Larson, K.L., et al., Sperm chromatin structure assay parameters as predictors of failed pregnancy following assisted reproductive techniques. Hum Reprod, 2000. 15(8): p. 1717-22.

8. Pfeifer, S., The clinical utility of sperm DNA integrity testing: a guideline (vol 99, pg 673, 2013). Fertility and Sterility, 2014. 101(3): p. 884-884.

9. Med, A.S.R., The clinical utility of sperm DNA integrity testing. Fertil Steril, 2008. 90: p. S178-80.

10. Swain, J.E., et al., Thinking big by thinking small: application of microfluidic technology to improve ART. Lab Chip, 2013. 13(7): p. 1213-24.

11. Tasoglu, S., et al., Exhaustion of Racing Sperm in Nature-Mimicking Microfluidic Channels During Sorting. Small, 2013. 9(20): p. 3374-84.

12. Nosrati, R., et al., Rapid selection of sperm with high DNA integrity. Lab Chip, 2014. 14(6): p. 1142-50.

13. Cho, B.S., et al., Passively driven integrated microfluidic system for separation of motile sperm. Anal Chem, 2003. 75(7): p. 1671-5.

14. Matsuura, K., et al., Screening of sperm velocity by fluid mechanical characteristics of a cyclo-olefin polymer microfluidic sperm-sorting device. Reprod Biomed Online, 2012. 24(1): p. 109-15.

15. Seo, D.B., et al., Development of sorting, aligning, and orienting motile sperm using microfluidic device operated by hydrostatic pressure. Microfluid Nanofluid, 2007. 3(5): p. 561-70.

16. Qiu, T., et al. A microfluidic "treadmill" for sperm selective trapping according to motility classification. in Solid-State Sensors, Actuators and Microsystems Conference (transducers), 2011 16th International. 2011.

17. Lopez-Garcia, M.D., et al., Sperm motion in a microfluidic fertilization device. Biomed Microdevices, 2008. 10(5): p. 709-18.

18. Fuhr, G., et al., High-frequency electric field trapping of individual human spermatozoa. Hum Reprod, 1998. 13(1): p. 136-41.

19. Ohta, A.T., et al., Motile and non-motile sperm diagnostic manipulation using optoelectronic tweezers. Lab Chip, 1998. 10(23): p. 3213-7.

20. Frimat, J.P., et al., Make it spin: individual trapping of sperm for analysis and recovery using micro-contact printing. Lab Chip, 2014. 14(15): p. 263541.

21. Parrilla, I., et al., Fluorescence in situ hybridization in diluted and flow cytometrically sorted boar spermatozoa using specific DNA direct probes labelled by nick translation. Reproduction, 2003. 126(3): p. 317-25.

22. Sarrate, Z. and E. Anton, Fluorescence in situ hybridization (FISH) Protocol in Human Sperm. J Vis Exp, 2009. 31: e1405.

23. Saravia, F., et al., Differences in boar sperm head shape and dimensions recorded by computer-assisted sperm morphometry are not related to chromatin integrity. Theriogenology, 2007. 68(2): p. 196-203. 
24. Lee, S. and J. Voros, An aqueous-based surface modification of poly(dimethylsiloxane) with poly(ethylene glycol) to prevent biofouling. Langmuir, 2005. 21(25): p. 11957-62.

25. Lee, S. and N.D. Spencer, Adsorption properties of poly(I-lysine)-graftpoly(ethylene glycol) (PLL-g-PEG) at a hydrophobic interface: influence of tribological stress, $\mathrm{pH}$, salt concentration, and polymer molecular weight. Langmuir, 2008. 24(17): p. 9479-88.

26. Marzec-Wroblewska, U., P. Kaminski, and P. Lakota, Influence of chemical elements on mammalian spermatozoa. Folia Biol, 2012. 58(1): p. 7-15.

27. Regehr, K.J., et al., Biological implications of polydimethylsiloxane-based microfluidic cell culture. Lab Chip, 2009. 9(15): p. 2132-9.

28. Calogero, A.E., et al., Sperm aneuploidy in infertile men. Reprod Biomed Online, 2003. 6(3): p. 310-7.

29. Egozcue, S., et al., Human male infertility: chromosome anomalies, meiotic disorders, abnormal spermatozoa and recurrent abortion. Hum Reprod Update, 2000. 6(1): p. 93-105.

30. Rubio, C., et al., Incidence of sperm chromosomal abnormalities in a risk population: relationship with sperm quality and ICSI outcome. Hum Reprod, 2001. 16(10): p. 2084-92.

31. Esteves, S.C. and S. Verza Jr., Relationship of in Vitro Acrosome Reaction to Sperm Function: An Update. Open Reprodu Sci J, 2011. 3(1): p. 72-84.

32. Katsuki, T., et al., Prediction of outcomes of assisted reproduction treatment using the calcium ionophore-induced acrosome reaction. Hum Reprod, 2005. 20(2): p. 469-75.

33. Sasaki, H., et al., Parylene-coating in PDMS microfluidic channels prevents the absorption of fluorescent dyes. Sensor Actuat B-Chem, 2010. 150(1): p. 478-82.

34. Li, N., et al., Confocal Raman micro-spectroscopy for rapid and label-free detection of maleic acid-induced variations in human sperm. Biomed Opt Express, 2014. 5(5): p. 1690-9.

35. Mallidis, C., et al., In situ visualization of damaged DNA in human sperm by Raman microspectroscopy. Hum Reprod, 2011. 26(7): p. 1641-9.

36. Malleo, D., et al., Continuous differential impedance spectroscopy of single cells. Microfluid Nanofluid, 2010. 9(2-3): p. 191-8.

37. Sun, T. and H. Morgan, Single-cell microfluidic impedance cytometry: a review. Microfluid Nanofluid, 2010. 8(4): p. 423-43. 
92 CHAPTER 4 


\section{Electrical analysis of single sperm motility}

An inevitable consequence of the declining male fertility is an increasing demand for assisted reproduction treatments such as in vitro fertilization and intra cytoplasmic sperm injection. Especially, ICSI has won popularity for treatment using semen samples with a very low sperm cell count. Currently, sperm selection for ICSI is performed manually using visual inspection of the sperm motility and morphology. Since only a single sperm cell is selected for injection, the success rate of fertilization is highly dependent on sperm selection. By performing this sperm inspection and selection in an automated fashion, treatment outcome might be improved. Intendingly, we report upon a microfluidic system which is able to entrap single sperm cells and allows non-invasive analysis of their motility on the single cell level by an electrical method.
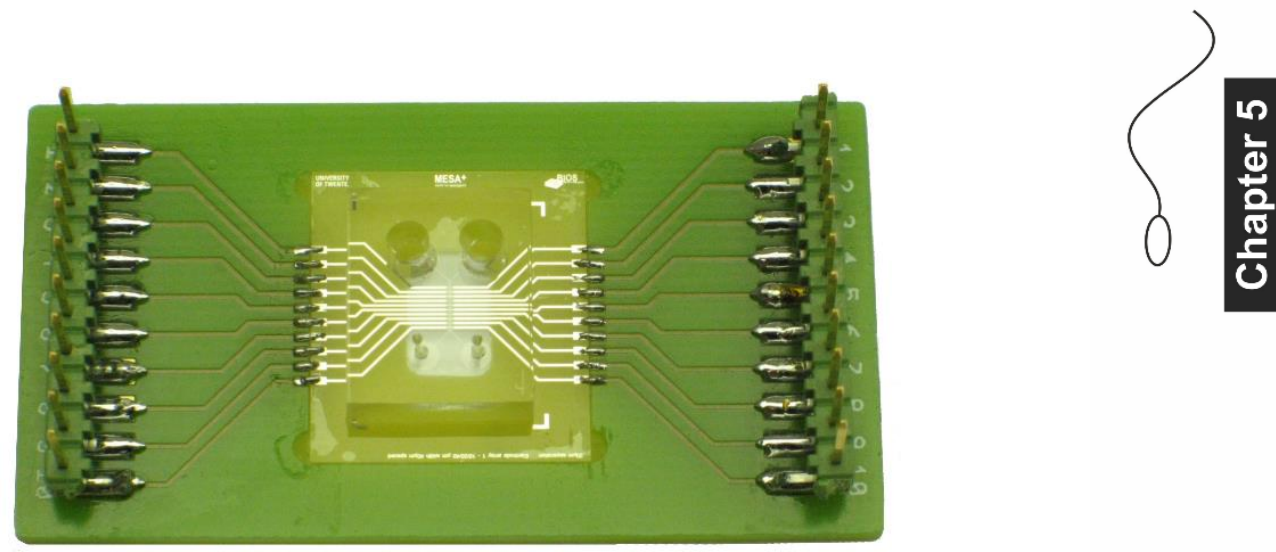

Photo of the glass-PDMS setup used for the electrical characterization of entrapped sperm cells.

Adapted from: de Wagenaar, B., Geijs, D.J., de Boer, H.L., Bomer, J.G., Olthuis, W., van den Berg, A., Segerink, L.I., Spermometer: Electrical characterization of single sperm motility. (submitted) 


\subsection{Introduction}

In the last decennium (2000-2010), the amount of IVF and ICSI treatments has approximately doubled [1, 2]. Especially, ICSI has won popularity for treatment of patients with no measurable sperm count (azoospermia) [3].

Sperm selection for ICSI treatments is based on visual inspection of the sperm motility and morphology. Various sperm characteristics such as DNA fragmentation [4], hyaluronic acid binding ability (HBA) [5] and hyperactivated motility (HAM) [6] have shown effect on IVF and ICSI outcome. Currently, no technologies are available in the clinic to assist the technician to choose and select the best motile spermatozoon. Advanced sperm selection might improve the pregnancy rate after ICSI treatment, which is approximately $30 \%[1,2]$.

Currently, few technologies have shown the potential to manipulate and analyse sperm cells on the single cell level. Fuhr et al. [7] showed to ability to entrap motile sperm cells using electric field cages at frequencies in the $\mathrm{MHz}$ range. This method was observed to have no influence on the cell viability when the cells were exposed to field strengths below $500 \mathrm{~V} \cdot \mathrm{cm}^{-1}$. Nascimento et al. [8] showed the potential to entrap single spermatozoa and to measure their motility using laser tweezers. No significant effect on sperm motility was observed for analysis times within $10 \mathrm{~s}$. Chen et al. [9] developed a microfluidic device for the analysis of single sperm motility. Using a resistive pulse method, impedance data was recorded which provided information on sperm motility when the sperm cells swam through a narrow aperture. In previous work in our group, we showed the ability to entrap single sperm cells using micro-contact printed protein spots [10]. After trapping single sperm cells, their motility was analysed using optical image analysis [10]. In a different approach, we used a hydrodynamic trapping procedure to entrap single sperm cells in small microfluidic channels [11]. Using this setup, cell characteristics such as the cell viability and acrosome integrity were investigated using fluorescent staining [11].

The previous reports showed the ability to analyse single sperm motility and/or entrap single sperm cells. However, these reports depend on optical methods, which limit their analysis throughput. To achieve a higher throughput, the amount of analysis/trapping sites on the microfluidic device must be increased, but more importantly, sperm analysis should be performed by means of an analysis technique which can be easily multiplexed. A potential approach is the investigation of sperm cells by impedance analysis using integrated microelectrodes. This 
technique has been used for analysis of both adherent cells [12] and cells in suspension [13], investigating cell properties such as cell size [14], cytoplasm conductivity $[15,16]$ and membrane potential and cell viability [17].

An important characteristic, which is used in the clinic for sperm selection, is the cell motility. Selection of sperm cells with high motility using both conventional [18] and microfluidic technologies $[19,20]$ have shown higher DNA integrity compared to the total unsorted population. High sperm DNA integrity is important for successful fertilization, since high degree of DNA fragmentation has shown a negative effect on ICSI outcome [4]. Furthermore, the amount of DNA fragmentation in sperm has been inversely related to its potential to hyperactivate [21], which additionally elaborates on a potential relation between the sperm swimming behaviour and DNA fragmentation. Although a variety of microfluidic systems have been developed for the selection of highly motile spermatozoa [19, 22-24], their application for single sperm selection for ICSI is limited.

Therefore, we propose a microfluidic system, which is capable of entrapping single spermatozoa and which is able to measure their motility using an analysis technique, which can be easily multiplexed. This system could be a potential tool for advanced sperm selection based on single sperm motility for ICSI applications.

\subsection{Materials \& Methods}

\subsubsection{Microfluidic setup \& chip fabrication}

The microfluidic setup consists of three different parts: a PDMS chip with trapping features, a glass chip with microelectrodes and a custom-made printed circuit board (PCB). Both chips were designed using CleWin software (version 4.0.1, WieWeb) and fabricated using standard photolithography techniques. The used microfluidic platform is shown in Fig. 5-1C. The microfluidic chip is illustrated in Fig. 5-3A, containing all relevant channel and electrode dimensions.

Fabrication of the SU-8 mold and PDMS chips is reported elsewhere [11]. In short, the SU-8 mold was fabricated by spinning and developing two layers of SU-8 (Microchem) on 4" silicon wafers. PDMS was fabricated (Sylgard 184, Dow Corning) using a 1:10 v/v ratio of base versus curing agent. After PDMS casting, curing and punching in- and outlets (Harris Uni-Core punchers, tip inner diameter 1.0 and 3.0 $\mathrm{mm}$, Ted Pella, Inc.), the chips were bonded to the glass chips using air plasma (Harrick PDC-001, NY, USA). The resulting microfluidic chips consisted of two main 
channels with a height of $20 \mu \mathrm{m}$, which are interconnected by $2 \mu \mathrm{m}$ wide and $1 \mu \mathrm{m}$ high microfluidic traps.

The glass chips were fabricated using a resist lift-off procedure on $500 \mu \mathrm{m}$ thick 4" Borofloat wafers (BF33). First, a $150 \mathrm{~nm}$ deep BHF recess wet- etch was performed after applying and developing the layer of photoresist. After etching, $20 \mathrm{~nm}$ tantalum and $130 \mathrm{~nm}$ platinum were sputtered onto the wafers. Subsequently, the resist and the excess metal were stripped off using acetone in an ultrasound bath. Finally, the wafers were cleaned and diced to the right size using a Disco DAD 321 dicer (Giorgio technologies).

The microfluidic setup was assembled by mounting a glass chip within the milledout section of the custom-made PCB. Subsequently, the microelectrodes on-chip were soldered to the connection pads on the PCB. A custom-made alignment setup was used to align the PDMS and glass chips (Fig. 5-1A). Using an adapted XYZ-table, PDMS chips were picked up using a needle array (Fig. 5-1BC) and bonded to the
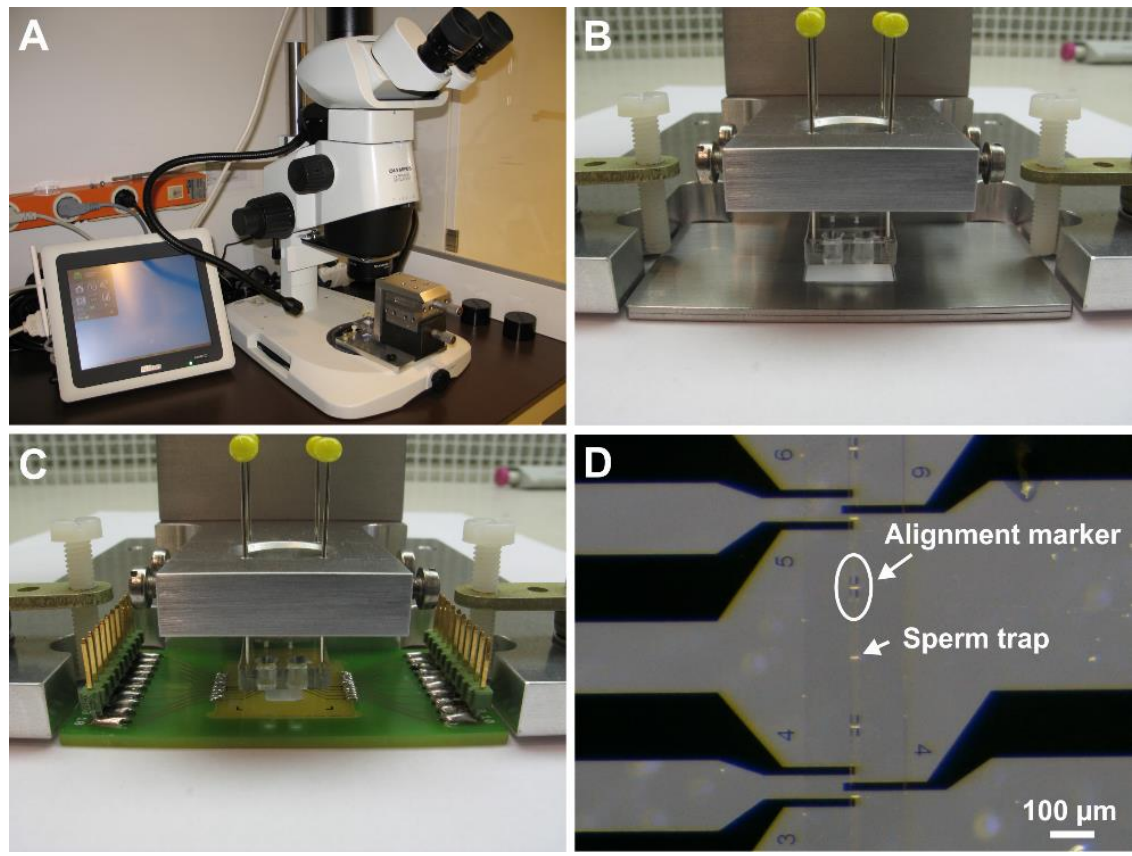

Fig. 5-1: Alignment setup for PDMS and glass chips. A) Using a stereo microscope and B) an adapted XYZ-table, B\&C) PDMS chips were pickedup with a needle array and D) aligned to the microelectrodes on the glass chips using special alignment markers. 
glass substrates (alignment within $5 \mu \mathrm{m}$ offset) after aligning the specially-designed alignment markers using an Olympus stereo microscope (Fig. 5-1D). After bonding, the finished setup was placed in an oven at $60^{\circ} \mathrm{C}$ for at least $30 \mathrm{~min}$ to improve adhesion between the PDMS and glass substrates.

\subsubsection{PLL-g-PEG surface coating}

To prevent cell adhesion during experiments, PDMS and glass surfaces were coated with poly(L-lysine)-grafted-poly(ethylene glycol) (PLL(20)-g[3.5]-PEG(2), SuSoS). PLL-g-PEG was rinsed through PDMS microchannels at a concentration of 100 $\mu \mathrm{g} \cdot \mathrm{mL}^{-1}$ in DI water for at least $15 \mathrm{~min}$.

\subsubsection{Sample preparation}

Fresh boar semen was obtained from a local artificial insemination centre ("KI Twenthe", Fleringen, The Netherlands) at a concentration of $20 \cdot 10^{6}$ cells $\cdot \mathrm{mL}^{-1}$. The samples were diluted with Beltsville Thawing Solution (BTS, Solusem, Aim Worldwide) to a concentration of $2 \cdot 10^{6}$ cells $\cdot \mathrm{mL}^{-1}$. Boar sperm cells have the following dimensions: head length $= \pm 9 \mu \mathrm{m}$ [25], head width $= \pm 4.5 \mu \mathrm{m}$ [25], head volume $= \pm 12.5 \mathrm{fL}$ [26], tail length $= \pm 45 \mu \mathrm{m} \mathrm{[27]}$.

\subsubsection{Hydrodynamic bead \& sperm trapping}

The hydrodynamic trapping procedure of sperm cells is described elsewhere [11]. In short, 3 and $4 \mu \mathrm{m}$ polystyrene beads $(\varnothing=4.17 \pm 0.03$ and $\varnothing=2.9 \pm 0.083 \mu \mathrm{m}$, Polysciences Inc.) and sperm cells were trapped within the interconnecting channels in between the two main channels of the PDMS chips. By generating a higher fluid flow in the bottom channel, compared to the flow in the bead- or sperm-loaded top channel ( 0.025 and $5 \mu \mathrm{L} \cdot \mathrm{min}^{-1}$, respectively), a fluid flow is created from the top to the bottom channel, which entrapped the sperm cells heador tail-first. After trapping, the flow rate in the top channel was reduced to 0.01 $\mu \mathrm{L} \cdot \mathrm{min}^{-1}$.

\subsubsection{Temperature logging}

Temperature data was recorded using a NI-USB-TC01 (National Instruments) equipped with a K-type thermocouple at a measurement interval of $1 \mathrm{~s}$. The thermocouple was taped to the glass to measure the temperature at the top of the glass substrate. 


\subsubsection{Chemical stimulation}

The swimming behaviour of entrapped spermatozoa was manipulated by the exposure to caffeine. After cell trapping and during continuous data recording, 10 $\mu \mathrm{L}$ of sperm diluent with $4 \mathrm{mM}$ caffeine was added to the channel inlet (containing $10 \mu \mathrm{L}$ sperm solution), yielding a final concentration of approximately $2 \mathrm{mM}$ caffeine. In total, two entrapped sperm cells were exposure to caffeine. Reports in literature showed an increase of sperm motility due to caffeine exposure [28, 29].

\subsubsection{Video acquisition \& optical data analysis}

Optical data was recorded using a Nikon TE2000-U microscope equipped with a 10x phase contrast objective and a Basler acA780-75 camera at $75 \mathrm{fps}$. For high quality videos, images were stored uncompressed using Pylon Viewer software. For videos longer than $20 \mathrm{~s}$, image compression was applied. For the analysis of the sperm beat frequency, a custom-built Matlab script was used. In short, this script is capable of tracking the sperm tail at a fixed distance with respect to the channel wall and records the position of the tail when the cells moves from left to right and vice versa. This position (i.e. X-location) is expressed with respect to the centre of the two measurement electrodes. The interelectrode distance between these two electrodes is $15 \mu \mathrm{m}$ and the X-location of the centre is defined as $0 \mu \mathrm{m}$. After calibrating the optical data using the interelectrode distance (approximately 0.55 $\mu \mathrm{m} \cdot$ pixel $\left.^{-1}\right)$, the sperm tail is tracked in between -7.5 and $7.5 \mu \mathrm{m}$. After plotting the position over time, frequency components within the signal were analysed by performing a Fast Fourier Transform (FFT) to determine the beat frequency.

\subsubsection{Differential impedance analysis and data analysis}

Impedance was recorded using an impedance spectroscope (HF2IS, Zurich Instruments, Zurich, Switzerland) equipped with a preamplifier (HF2TA, Zurich Instruments). A $1 \mathrm{MHz}$ AC signal with an amplitude of $0.5 \mathrm{~V}$ was generated on output 1 , which was connected to the exciting electrode of the electrode array (Fig. 5-2A). The two sensing electrodes were connected to input 1 and 2 of the HF2IS via the HF2TA and used to measure the impedance, differentially (Fig. 5-2B). Impedance was recorded using a bandwidth of $200 \mathrm{~Hz}$ and a sampling frequency of $899 \mathrm{~Hz}$. Recorded differential impedance data was imported and processed in Matlab (R2014b, MathWorks). The differential impedance response (Fig. 5-2E) was obtained by subtracting the recorded impedance at input 1 (control, Fig. 5-2C) from the impedance at input 2 (cell trap, Fig. 5-2D). Analysis of the sperm motility in the frequency domain was performed after performing a FFT of the differential signal. 
The amplitude of the motility-related oscillation was measured using peak detection.

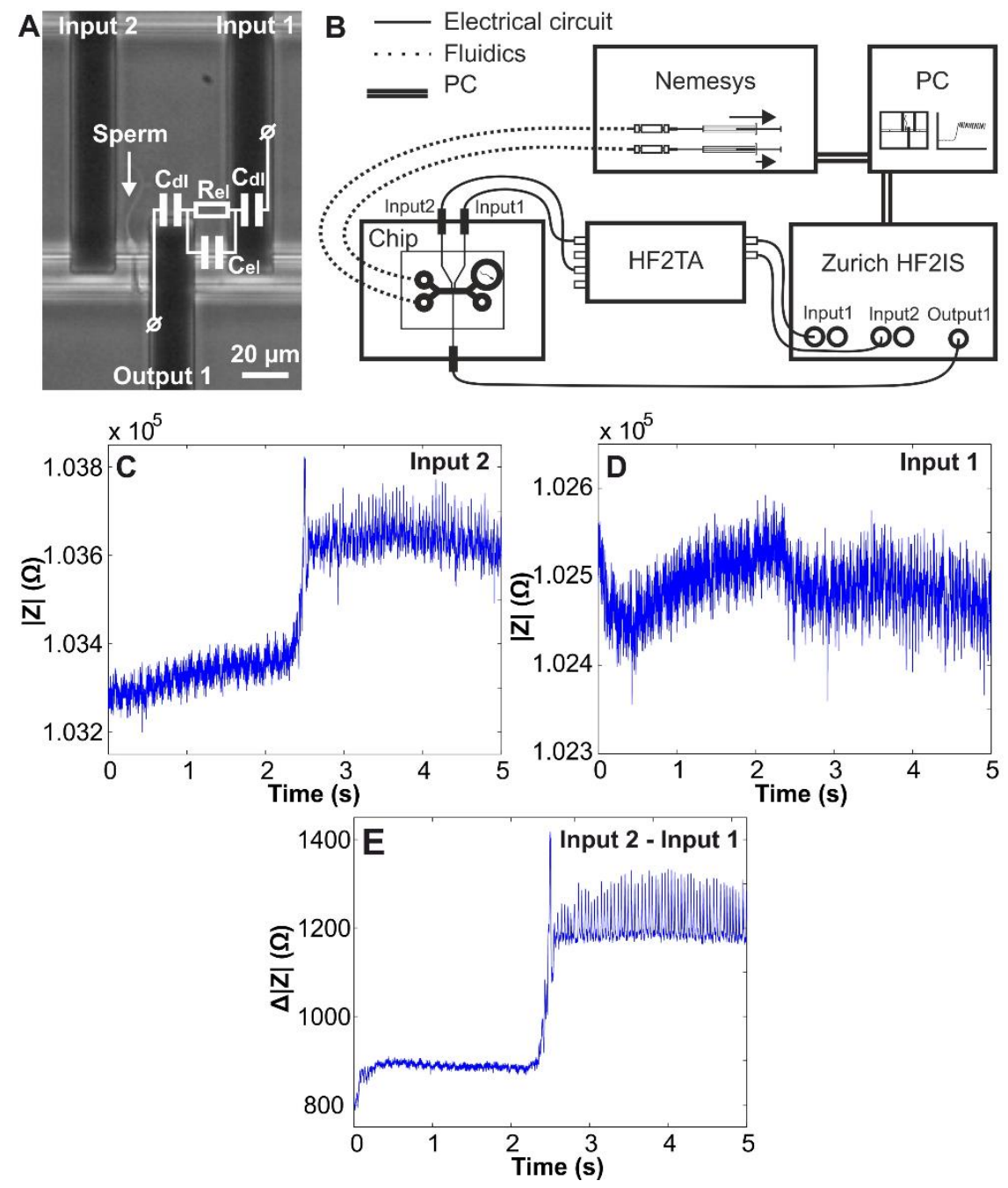

Fig. 5-2: Electrical analysis of single sperm motility. A) Microfluidic chip including ECM for an empty trap. B) Illustration of the electrical setup. The microelectrodes for data recording are connected to input 1 and 2 of the impedance spectroscope via the preamplifier; a sinusoidal excitation is applied to the microelectrode connected to output 1 . C) The recorded impedance at input 1 is subtracted from $D$ ) the impedance recorded at input 2 to obtain $\mathrm{E}$ ) the differential impedance response. 
To investigate the impedance response of the microfluidic device as a function of frequency, the impedance was recorded simultaneously at input 1 and 2 using a custom-built LabVIEW program when sweeping frequency between $100 \mathrm{~Hz}$ and 5 $\mathrm{MHz}$.

\subsubsection{Computer assisted sperm analysis}

A computer assisted sperm analysis (CASA) system (Sperm Class Analyser v5.4.0.0, Microoptic) was used to record the beat cross frequency of a population of sperm cells. Before CASA analysis of the sperm motility in $3 \mu \mathrm{L}$ Leja-20 slides pre-warmed on a microscope hot plate, a $20 \cdot 10^{6}$ sperm cells $\cdot \mathrm{mL}^{-1}$ solution was warmed up to 37 ${ }^{\circ} \mathrm{C}$ in a water bath during $15 \mathrm{~min}$.

\subsubsection{COMSOL simulation}

A COMSOL (v4.4) model was constructed to simulate the electrical field in the microfluidic device. An electrical currents (ec) model was used to simulate the electrical potential and normalized electrical field strength in between the electrodes in a 3D model when assuming a high conductive electrolyte $\left(1.4 \mathrm{~S} \cdot \mathrm{m}^{-1}\right)$ and applying $0.5 \mathrm{~V}$ to the excitation electrode.

\subsection{Results \& discussion}

\subsubsection{Device design \& characterization}

The microfluidic setup consists of two separate parts: a PDMS chip containing the fluidic features and a glass chip containing the planar microelectrodes (Fig. 5-1C). The fluidic features consist of two $100 \mu \mathrm{m}$ wide main channels, which are separated by a $20 \mu \mathrm{m}$ wide PDMS ridge. Within this ridge, 20 side channels with a width of 2 $\mu \mathrm{m}$ and a height of $1 \mu \mathrm{m}$ interconnected the main channels, which act as cell traps when a pressure gradient is created between the main channels. At either sides of the cell trap, $20 \mu \mathrm{m}$ wide electrodes are positioned to measure the change in impedance upon sperm entrapment. The distance between these electrodes is 15 $\mu \mathrm{m}$. In order to entrap a sperm cell in between the electrodes, the alignment of the glass and PDMS chips must be precise. A custom-made XYZ table equipped with a needle pick-up array was used to perform chip alignment within a precision of $5 \mu \mathrm{m}$ (Fig. 5-1).

Besides the electrodes at either sides of a cell trap, an additional electrode is designed to perform a control measurement (Fig. 5-3A). A sinusoidal excitation is applied to the middle electrode, while the impedance is monitored simultaneously 

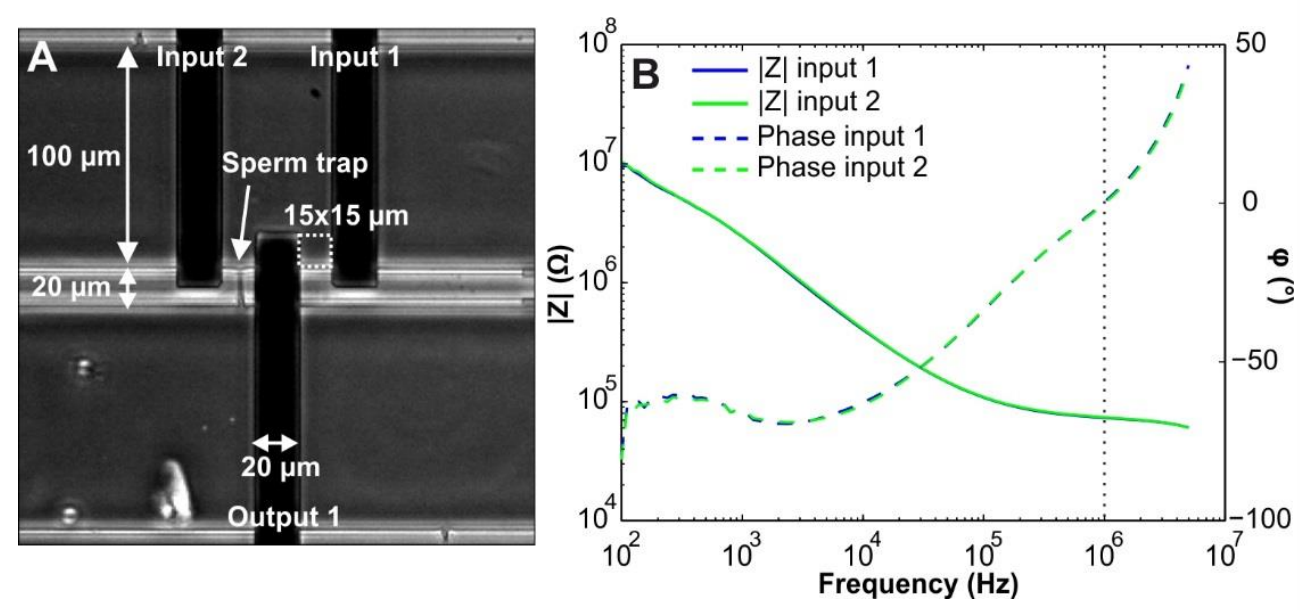

Fig. 5-3: A) The microfluidic chip consists of two main channels, interconnected by $2 \mu \mathrm{m}$ wide and $1 \mu \mathrm{m}$ high side channels, which act as cell traps. The electrode array consists of two sensing electrodes (input 1 and 2) and one excitation electrode (output 1), which were used for differential impedance analysis. B) The impedance and phase response of the microfluidic setup (without sperm cell) were investigated when sweeping frequency between $100 \mathrm{~Hz}$ and $5 \mathrm{MHz}$ and recording under physiological conditions $\left(\sigma_{e l}=1.4 \mathrm{~S} \cdot \mathrm{m}^{-1}\right)$ at input 1 and 2, separately.

at the cell trap region and control region using electrodes connected to input 2 and input 1 , respectively. This impedance is influenced by several components within the setup (Fig. 5-2A): the electrode-electrolyte interface $\left(C_{D L}\right)$, the electrolyte conductivity $\left(R_{e l}\right)$ and electrolyte capacitance $\left(C_{e l}\right)$. When introducing a sperm cell in between the electrodes, the impedance is further influenced by the cell membrane $\left(C_{m e m}\right)$ and the cell interior $\left(R_{i}\right)$. The electrical response of this setup (without sperm cell) was investigated by recording the impedance when sweeping the measurement frequency between $100 \mathrm{~Hz}$ and $5 \mathrm{MHz}$ to find the optimal value for continuous impedance monitoring (Fig. 5-3B).

A clear effect of $C_{D L}$ was observed at the low frequency region ( $<100 \mathrm{kHz}$ ). At frequencies around $1 \mathrm{MHz}$, the absolute impedance was dominated by $R_{e l}$ at which the impedance had a value of $100 \mathrm{k} \Omega$ and the phase shift was approximately $0^{\circ}$. This frequency was selected for impedance monitoring in all reported experiments. The electrical response measured at both inputs showed almost identical behaviour, indicating that the cell trap itself did not influence the electrical response of the setup to a big extend. Therefore, the recorded impedance at input 1 is suitable as an internal control for differential measurement. 


\subsubsection{Bead \& sperm trapping}

Beads and sperm cells were captured by a hydrodynamic trapping procedure as described in section 5.2.4. By continuous impedance monitoring, the impedance change upon bead and cell trapping was recorded. This change was determined after obtaining the differential impedance response. A typical trapping event of a 4 $\mu \mathrm{m}$ bead is shown in Fig. 5-4A. The impedance change of $4 \mu \mathrm{m}$ beads was $705 \pm$ $10.7 \Omega$ (mean \pm standard deviation, $n=10$ ) whereas the impedance change of $3 \mu \mathrm{m}$ beads was $210 \pm 8.5 \Omega(n=10)$. When expressing the impedance change per volume of entrapped particle, the impedance changes for 3 and $4 \mu \mathrm{m}$ beads were 16.4 $\Omega \cdot \mu \mathrm{m}^{-3}$ and $18.6 \Omega \cdot \mu \mathrm{m}^{-3}$, respectively.
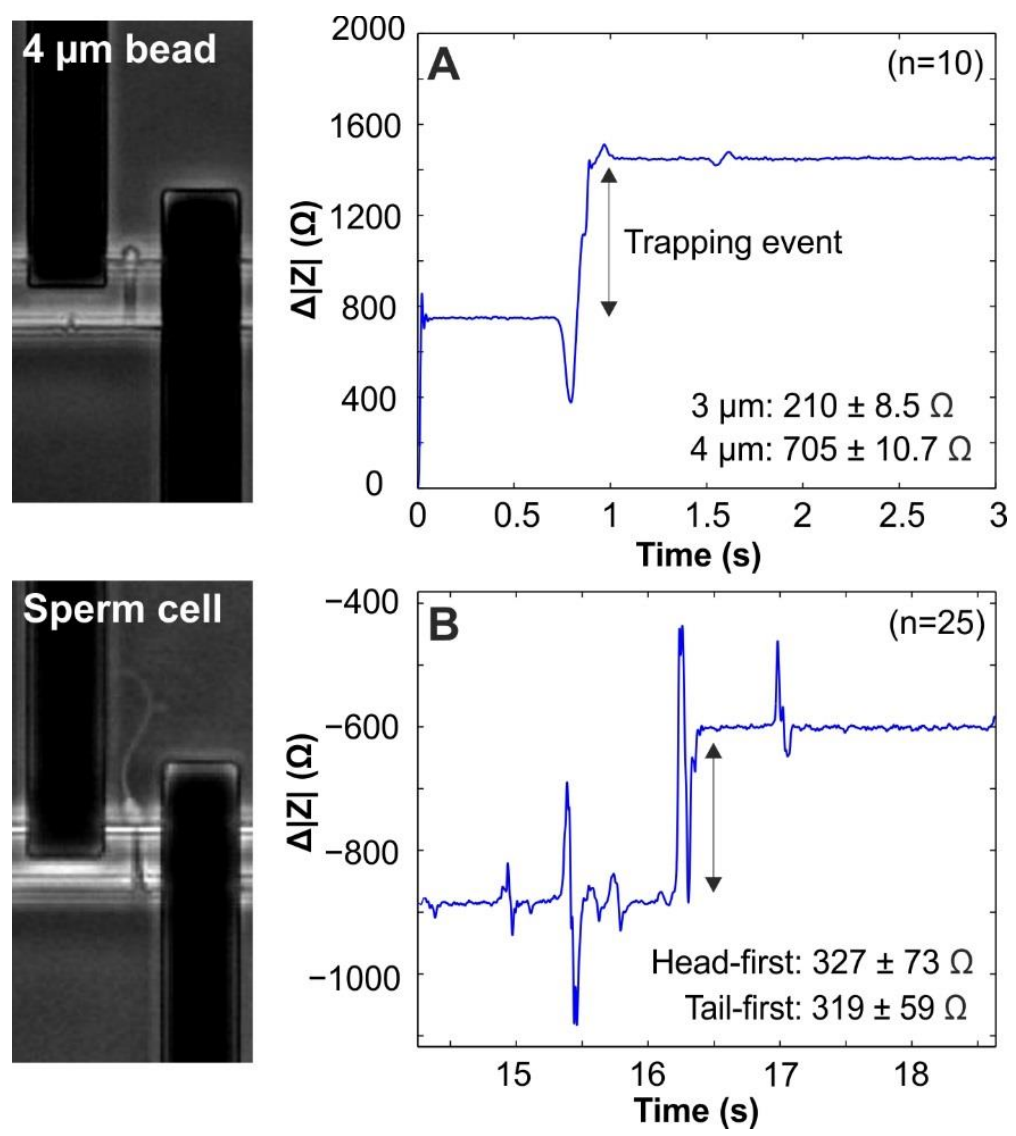

Fig. 5-4: A) Recorded impedance change upon trapping of 3 or $4 \mu \mathrm{m}$ beads $(n=10)$ and B) sperm cells in head-first or tail-first orientation ( $n=25$ ) (Values are reported in mean $\pm 1 x$ standard deviation). 
Entrapment of sperm cells resulted in a head-first or tail-first capture of the cell (Fig. 5-4B). At a measurement frequency of $1 \mathrm{MHz}$ in a high-conductive isotonic environment $\left(1.4 \mathrm{~S} \cdot \mathrm{m}^{-1}\right)$, the cell membrane acts as an insulator. As a result, the recorded impedance change increased upon cell entrapment. To find out whether the orientation had an effect on the recorded impedance, the impedance change was determined for head-first and tail-first trapped sperm cells. The recorded impedance of $327 \pm 73 \Omega(n=25)$ and $319 \pm 59 \Omega(n=25)$, respectively, showed no difference between the two orientations. When expressing this impedance change per volume, a value of approximately $26.2 \Omega \cdot \mu \mathrm{m}^{-3}$ is found. This value is significantly higher compared to those of the beads, which can be explained by the shape of the sperm cell.

Spermatozoa have a natural tendency to swim close to surfaces and to swim against small fluid flows [30-32]. This behaviour was also observed in our microfluidic system (results not shown). As a result, trapping spermatozoa head-first was rather straight-forward, because the sperm heads were positioned in close distance to the cell traps when swimming. Upon sperm entrapment, the recorded impedance data
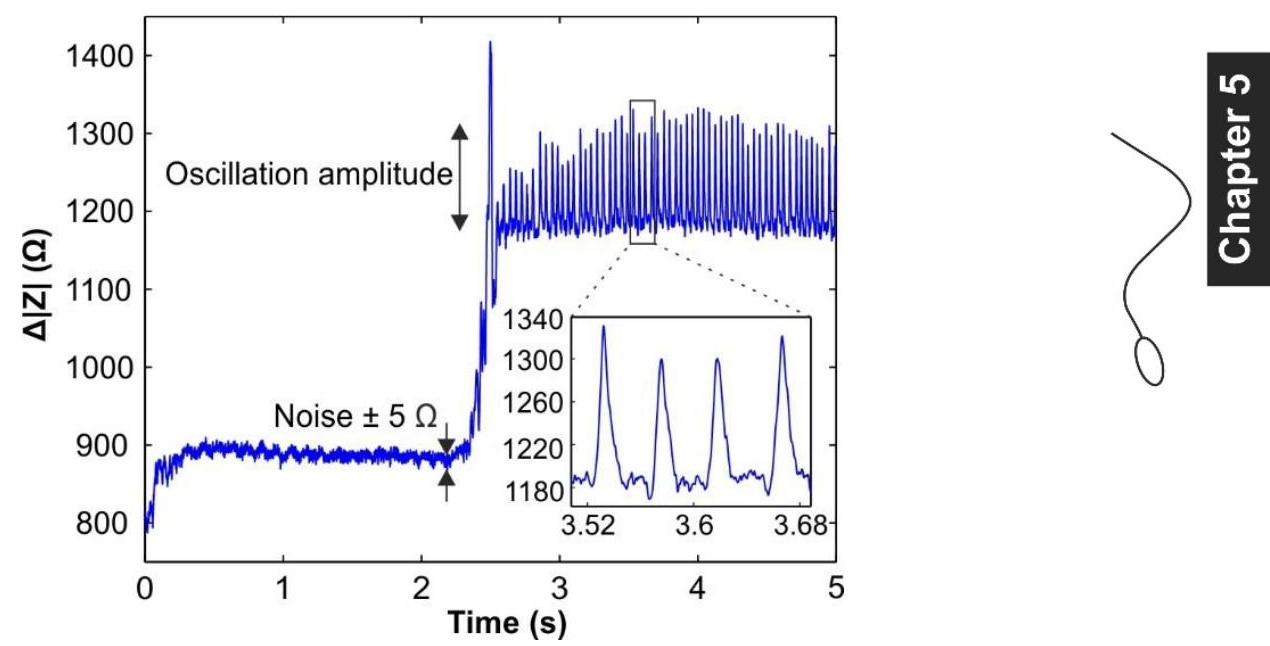

Fig. 5-5: Recorded impedance before and after trapping of a motile sperm cell. Before trapping, the absolute impedance value (differential) was approximately 900 $\Omega$. After trapping, this value increased to approximately $1200 \Omega$. A steady oscillation was observed in the impedance response after entrapment, which is caused by to the motile behaviour of the spermatozoon. 
showed an initial increase as previously shown (Fig. 5-4B). Interestingly after cell trapping, a repetitive oscillation in the differential impedance was observed with much larger amplitude compared to the recorded noise (Fig. 5-5). This oscillation was caused by the motile behaviour of the sperm cell. Entrapped sperm cells showed good cell motility over periods exceeding $15 \mathrm{~min}$, which indicates the noninvasive nature of the trap and analysis techniques. However, when the sperm head came in direct contact with one of the electrodes, the motility was observed to decline or the sperm cell was temporarily paralyzed (data not shown). This finding, combined with the simulated field strength of $>500 \mathrm{~V} \cdot \mathrm{cm}^{-1}$ close to the excitation electrode (Fig. 5-11B), are consistent with the observations by Fuhr et al. [7], describing sperm immobilization at field strengths of around $500 \mathrm{~V} \cdot \mathrm{cm}^{-1}$.

\subsubsection{Optical \& electrical analysis of sperm motility}

To investigate whether the recorded impedance provides information on sperm motility as previously suggested, the flagellar motion of a trapped cell in between the electrodes was recorded optically at a frame rate of $75 \mathrm{fps}$. Subsequently, a custom-built Matlab program was used to automatically track the sperm tail at a fixed height (Fig. 5-6A). This position, expressed as an X-location with respect to the electrode array's centre, described a motion along the X-axis (Fig. 5-6B \& Fig. 5-7A). When comparing the obtained optical data (Fig. 5-7B) with the electrical data (Fig. 5-7C), the oscillation in both signals showed a similar frequency. After performing a FFT, an identical base frequency was found in both the optical and electrical data (Fig. 5-7D), which was around $14.5 \mathrm{~Hz}$ for this particular cell. For all experiments reported in this chapter, the electrical determined sperm oscillation is called the sperm beat frequency. In literature, the sperm beat cross frequency (BCF) is a parameter which is defined as the frequency of the sperm head crossing the sperm average path [33,34], expressed as the amount of intersections (in either direction) per second $(\mathrm{Hz})$. In our reported experiments, the obtained frequency represents the amount of tail oscillations per second, in which a single oscillation is defined as the movement of the sperm tail from the first electrode towards the second electrode and back. In this case, in a single oscillation the sperm cell crossed its hypothetical path (i.e. centre line) twice. Therefore, the BCF of the entrapped spermatozoon is determined by multiplying the calculated beat frequency by a 

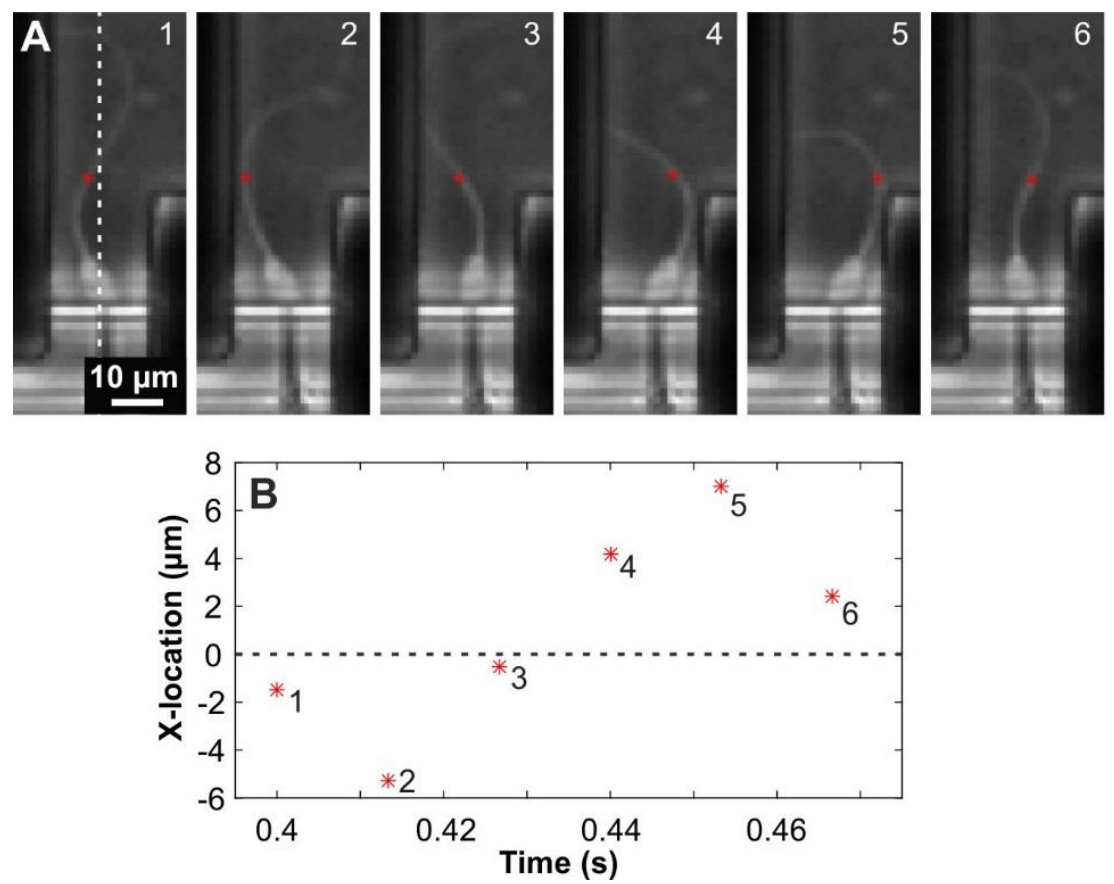

Fig. 5-6: A) Optical tracking of the sperm tail using a custom-built Matlab script. At each consecutive frame (at $75 \mathrm{fps}$ ) the sperm tail was tracked at a fixed distance with respect to the sperm trap and visualized with a red asterisk. The white dashed line indicates the centre line, which is positioned at the centre of the two electrodes. B) Subsequently, the location of the tracked tail can be plotted with respect the centre line, which is positioned at a X-location of $0 \mu \mathrm{m}$.

factor of 2, yielding a BCF of approximately $29 \mathrm{~Hz}$. This result was comparable to the average BCF of $31.5 \mathrm{~Hz}$ of a control sample, which was determined by CASA analysis.

\subsubsection{Temperature dependent sperm motility}

The previous section reported on the ability to measure the beat frequency of an entrapped sperm cell using differential impedance monitoring. To be able to distinguish between sperm cells with a good and bad motility, this technique must be able to quantify the swimming behaviour of sperm cells with varying motility. As a model system, we used an entrapped spermatozoon and decreased the temperature to mimic sperm cells with a reduced motility. Subsequently, the temperature was increased to observe the recovery of sperm swimming. 

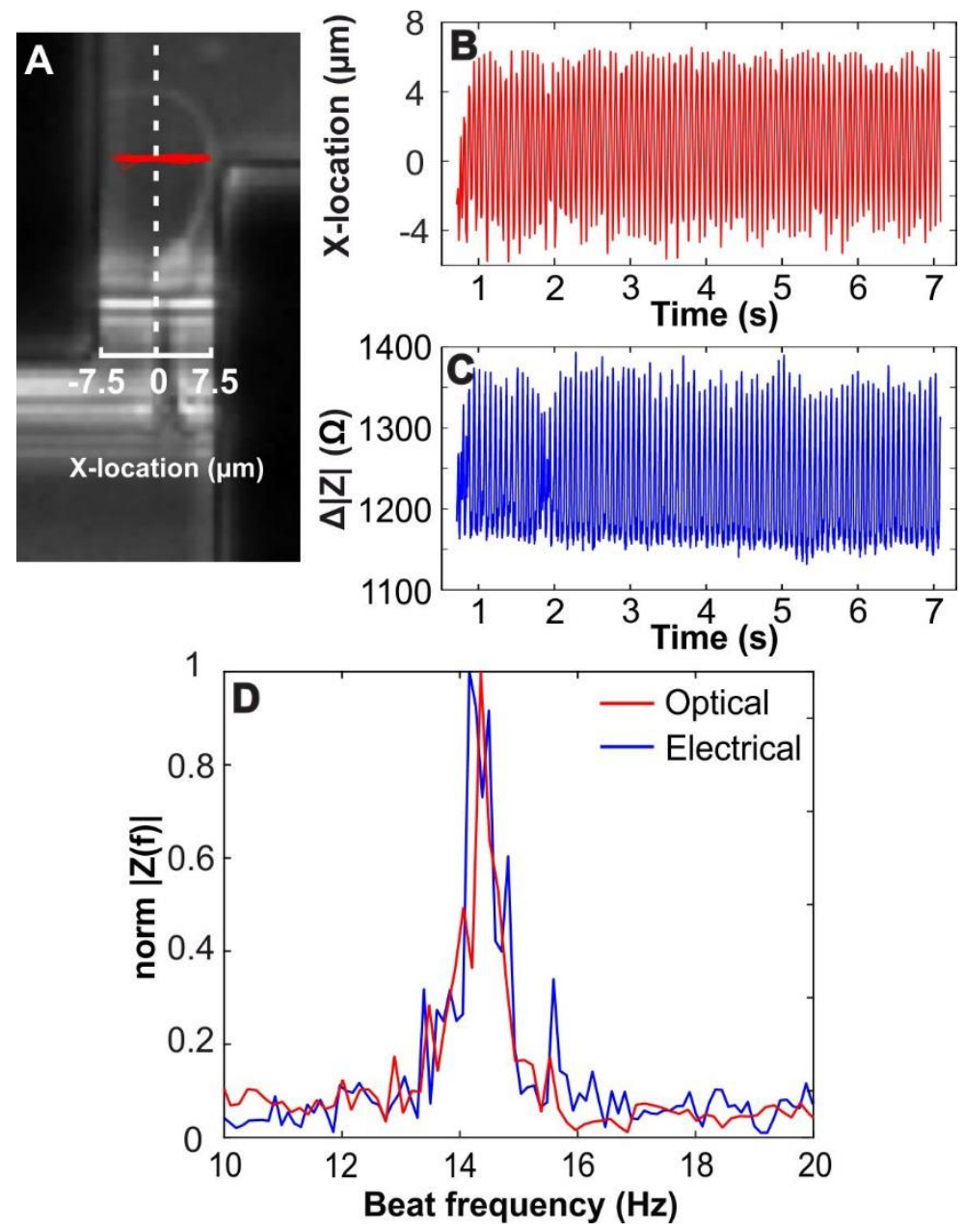

Fig. 5-7: A) The position of the tail of an entrapped spermatozoon was tracked using a custom-built Matlab program. B) When plotting the position of the tail (x-position) over time, an oscillation was observed that was comparable to $\mathrm{C}$ ) the electrical response. D) FFT analysis of both optical and electrical data showed an identical base frequency at approximately $14.5 \mathrm{~Hz}$.

After entrapping a motile sperm cell, the hot-plate was switched off ( $t=0 \mathrm{~s}$ ). At 5 $\mathrm{min}$, the hot-plate was switched on again and data was recorded up to $10 \mathrm{~min}$. The differential impedance data was used to construct a FFT every $5 \mathrm{~s}$. At $\mathrm{t}=0 \mathrm{~s}$, a frequency around $35 \mathrm{~Hz}$ was observed in the FFT. As the temperature decreased over time, a clear left shift of the base frequency was observed in the spectrum (Fig. $5-8 \mathrm{~A})$. After $5 \mathrm{~min}$, the motility reduced to frequencies around $15 \mathrm{~Hz}$. When the 
temperature started to increase after switching on the hot-plate, the measured frequency clearly shifted to the right. After $10 \mathrm{~min}$, the flagellar frequency recovered to a value between $30-35 \mathrm{~Hz}$ (Fig. 5-8B). This beat frequency was significantly higher compared to the beat frequency shown in Fig. 5-7D. This difference can be explained by a difference in intrinsic sperm motility between the two entrapped cells.
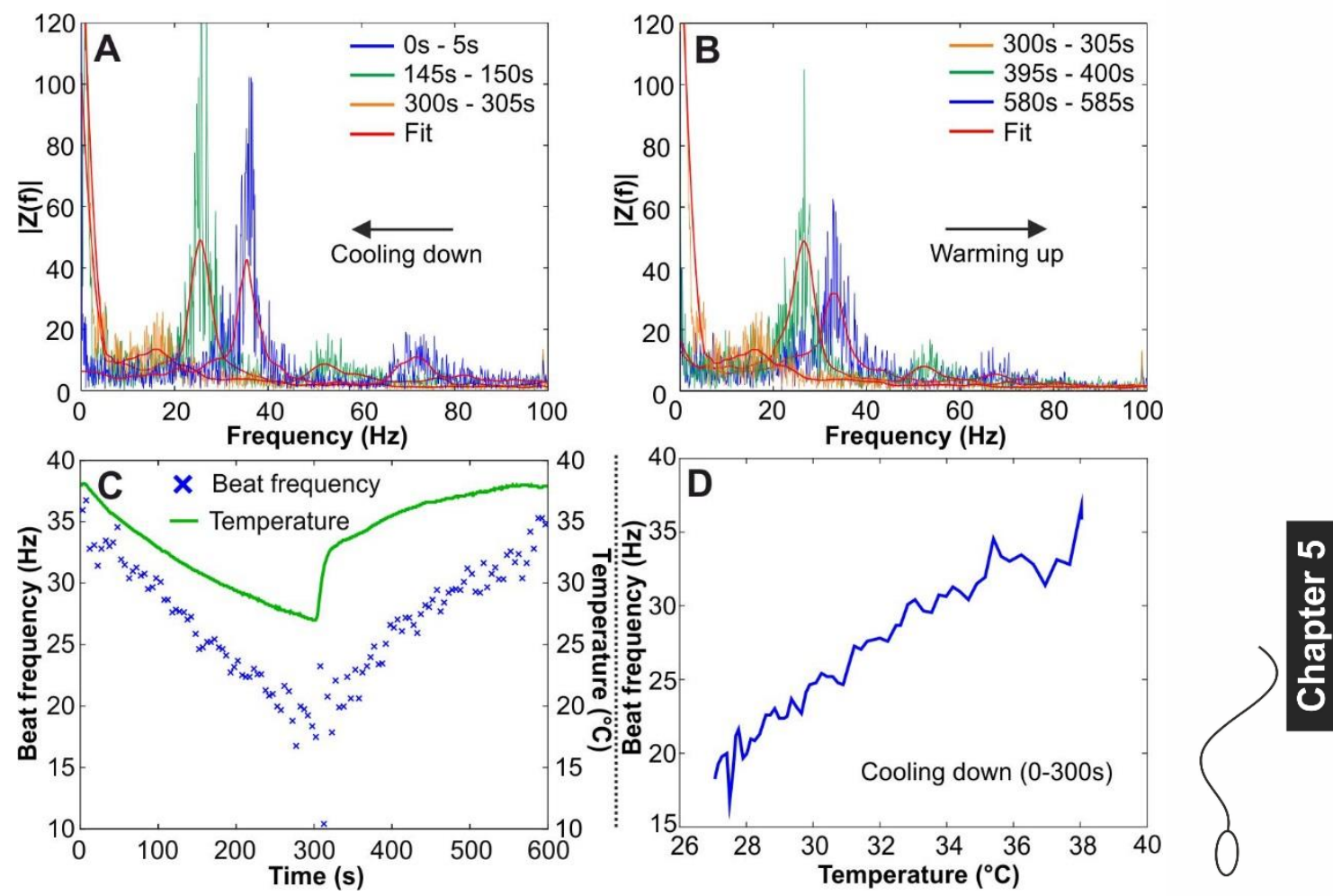

Fig. 5-8: The effect of temperature on sperm beat frequency. FFT analysis was performed on the absolute impedance using $5 \mathrm{~s}$ intervals. A) When the solution was cooling down, a decrease in base frequency was observed over time, which corresponds to the reduced flagellar beat frequency of the spermatozoon due to the decrease in temperature. B) Contrarily, the base frequency (i.e. beat frequency) was observed to increase over time when warming up the solution. C) The resulting trend in base frequency over time showed good correspondence with the measured temperature. D) The relation between base frequency and temperature (cooling down) showed a linear behaviour. 
A data fit was constructed for each separate FFT and the maximum of this fit was determined, representing the average beat frequency. This average frequency was plotted over time to show the declining and ascending trends in frequency (Fig. $5-8 \mathrm{C})$. Furthermore, the temperature on the surface of the glass chip was measured to show the trend in temperature $(n=1)$. The frequency was observed to gradually decrease over time in roughly a linear fashion. After switching on the hot-plate, the temperature was observed to increase rapidly, and interestingly, the increase in frequency showed a slight exponential trend. When considering a delay between the temperature of the glass (measured) and the temperature of the fluid in the microchannel (unknown), a good correspondence was found between the trends in temperature and sperm beat frequency. When plotting the temperature versus the measured frequency when the fluid is cooling down, a rough linear trend was observed (Fig. 5-8D).

\subsubsection{Chemical stimulation}

Various factors are known to influence sperm motility besides temperature, including the internal $\mathrm{pH}$ and ionic composition [35, 36]. Furthermore, several chemical compounds are known to alter the swimming behaviour of sperm including progesterone [37], caffeine [38] and calcium ionophore A23187 [39]. To show the effect of chemical stimulation on single sperm motility and to investigate whether this effect can be measured electrically, trapped spermatozoa were exposed to caffeine. Although moderate intake of caffeine did not show an effect on the sperm motility in a human population [40], direct exposure to this compound in vitro is known to increase the sperm motility [38].

After trapping a spermatozoon head-first, $4 \mathrm{mM}$ caffeine was added to the channel insert, yielding a final concentration of $\pm 2 \mathrm{mM}$. Before adding caffeine, the sperm cell showed a stable beat frequency at approximately $15 \mathrm{~Hz}$ (Fig. 5-9A). After adding the caffeine, the frequency did not change instantly. However, after an additional $30 \mathrm{~s}$, the beat frequency was observed to increase gradually to a maximum of 22 $\mathrm{Hz}$ (optically verified, results not shown). This offset could be caused by a delay between caffeine insertion and cell exposure due to slow mixing of fluids in the inlet well. Furthermore, the cellular response to caffeine exposure may not have been instant. Interestingly, the total amplitude of the signal decreased over time. Before the beat frequency started to increase, the signal amplitude was approximately 250 $\Omega$, whereas this value dropped under $150 \Omega$ at the end of the experiment. Possibly, this decrease is related to the decline in flagellar beat angle (FBA). 

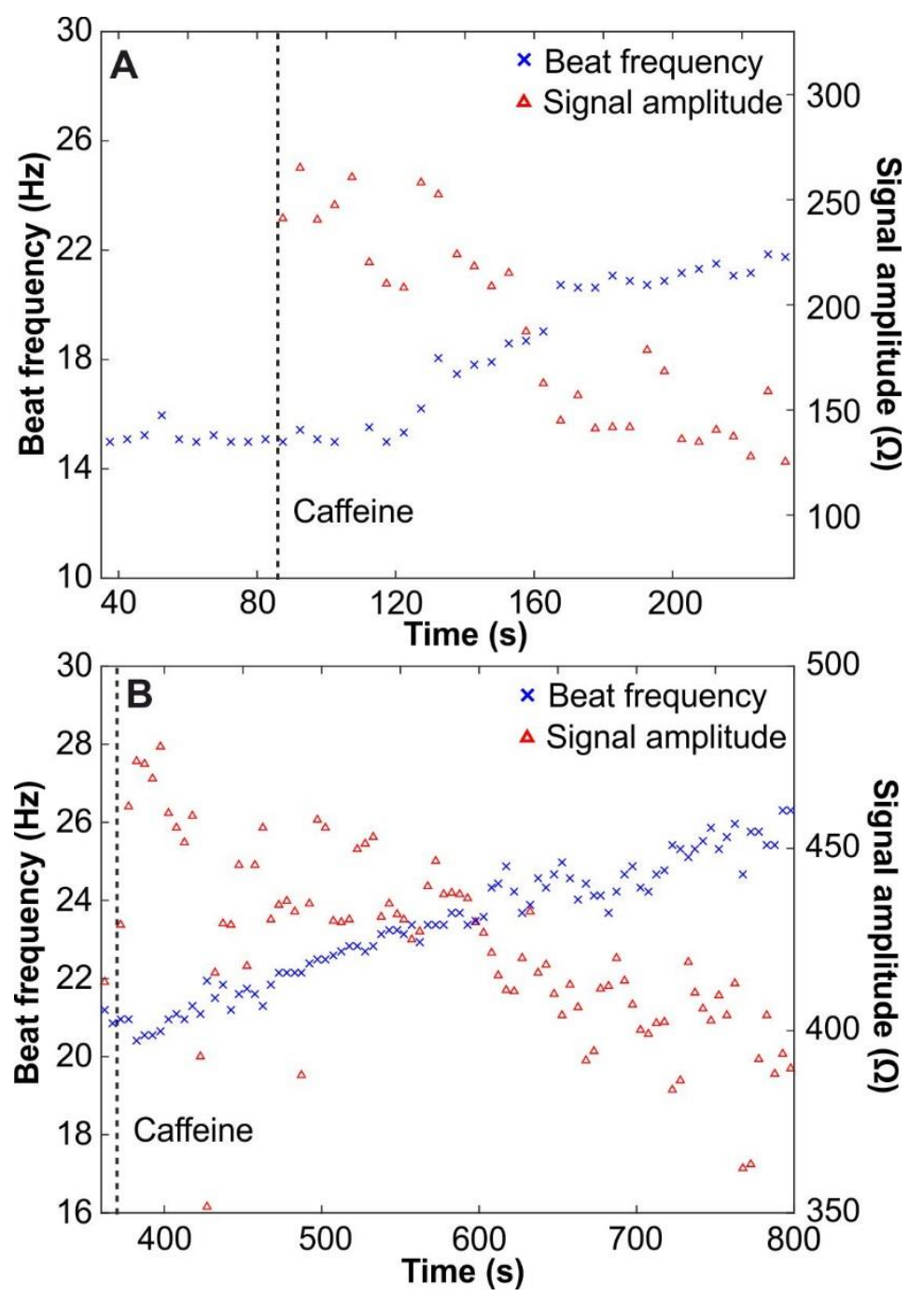

Fig. 5-9: The effect of caffeine on the beat frequency and oscillation amplitude. A) After cell entrapment at $35 \mathrm{~s}$, a steady beat frequency was observed of approximately $15 \mathrm{~Hz}$. After introducing caffeine at $86 \mathrm{~s}$, a steady increase of the beat frequency to approximately $22 \mathrm{~Hz}$ was observed, starting at $120 \mathrm{~s}$. B) For a second spermatozoon, the beat frequency and oscillation amplitude were investigated over time. After cell entrapment ( $360 \mathrm{~s}$ ) and caffeine exposure (370 s), the beat frequency was observed to increase from $20-21 \mathrm{~Hz}$ to $28-29 \mathrm{~Hz}$. The amplitude of the oscillation was observed to decrease over time from $450-500 \Omega$ to values between 150-200 $\Omega$. This decrease in amplitude was likely caused by the decrease in flagellar beat angle. 
When the beat frequency of the cell increased, the angle of the beating flagellum decreased simultaneously (optically verified, results not shown). Correspondingly, the total displacement of the sperm head with respect to the X-axis started to decrease over time, i.e. the distance between the sperm head and the edges of the electrodes started to increase. As a result, the signal amplitude decreased. In a second experiment, similar effects on the beat frequency and the motility-related signal amplitude were observed by exposing an entrapped sperm cell to caffeine (Fig. 5-9B). At the start of the experiment, a frequency of $20-21 \mathrm{~Hz}$ and a signal amplitude of $\pm 475 \Omega$ were recorded. This frequency increased to a maximum of 28 $29 \mathrm{~Hz}$ and the amplitude decreased to values under $400 \Omega$ after caffeine exposure (Fig. 5-9B). This preliminary data showed a potential relation between flagellar beat angle and the amplitude of the motility-related oscillation.

\subsubsection{Hyperactivation}

Hyperactivation is an important phenomenon, which aids the sperm cell to migrate to and penetrate trough the zona pellucida of the egg cell. During hyperactivation, spermatozoa exhibit an asymmetric beat pattern with increased flagellar amplitude. Research have shown a decrease in hyperactivation potential upon increase of DNA fragmentation [21]. Since DNA fragmentation has shown a negative effect on ICSI outcome [4], the potential to distinguish and select hyperactivated spermatozoa from non-hyperactivated spermatozoa, might improve treatment outcome.

Hyperactivated motility is characterized by asymmetric tail beating, in which the flagellar beat angle is increased and the flagellar beat frequency is decreased [41, 42]. Previous results have shown the potential to investigate the beat frequency. Furthermore, exposure to caffeine resulted in increased sperm beat frequency and decreased signal amplitude, which was likely caused by a decrease in beat angle. To investigate the potential to electrically distinguish between non-hyperactivated and hyperactivated spermatozoa, the beat angle, frequency and signal amplitude were measured of spermatozoa with distinct swimming behaviour on a single chip. In most occasions, spermatozoa were trapped that showed symmetrical tail beating before entrapment and which swam close to the channel wall (results not shown). An example is shown in Fig. 5-10A. During entrapment, a FBA of $59 \pm 11^{\circ}$ (Fig. 5-10A) and a BCF of $36 \mathrm{~Hz}$ (Fig. 5-10B) were measured. This data is consistent for a sperm cell which is non-hyperactivated [41]. 

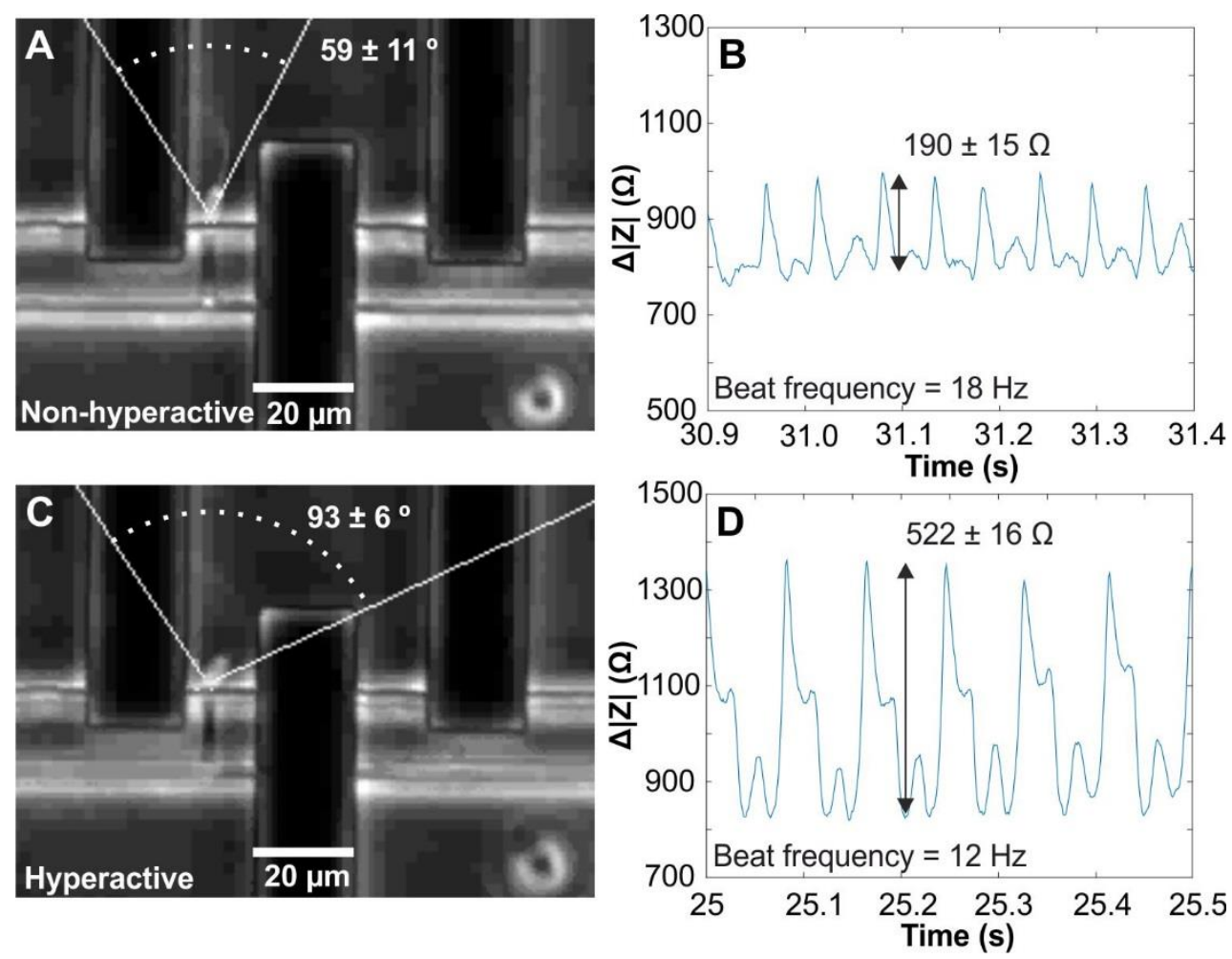

Fig. 5-10: Relation between FBA and oscillation amplitude. In two separate experiments, two spermatozoa were entrapped, in which one of them was hyperactivated. The beat frequency, FBA and oscillation amplitude were investigated at a fixed interval of $0.5 \mathrm{~s}$. A\&C) The FBA of a hyperactive spermatozoon was higher compared to a non-hyperactive spermatozoon (59 \pm $11^{\circ}$ and $93 \pm 6^{\circ}$, respectively). B\&D) Correspondingly, the beat frequency was smaller for the hyperactivated spermatozoon ( $12 \mathrm{~Hz}$ compared to $18 \mathrm{~Hz}$ ). As a result of smaller flagellar beat angle, the recorded oscillation amplitude of the hyperactivated spermatozoon was higher compared to the non-hyperactived spermatozoon ( $522 \pm 16 \Omega$ and $190 \pm 15 \Omega$, respectively).

In a rarer occasion, a sperm cell was trapped, which showed asymmetrical tail beating, pushing the cell away from the channel wall. After entrapment, the spermatozoon showed hyperactivated motility, in which the FBA was $93 \pm 11^{\circ}$ (Fig. 5-10C) and the BCF was $24 \mathrm{~Hz}$ (Fig. 5-10D). The signal amplitude of the hyperactive spermatozoon ( $522 \pm 16 \Omega$ ) was significantly higher compared to the amplitude of the non-hyperactivated spermatozoon $(190 \pm 15 \Omega)$. The increase in amplitude is in agreement with the increase of FBA for a hyperactivated sperm cell. 


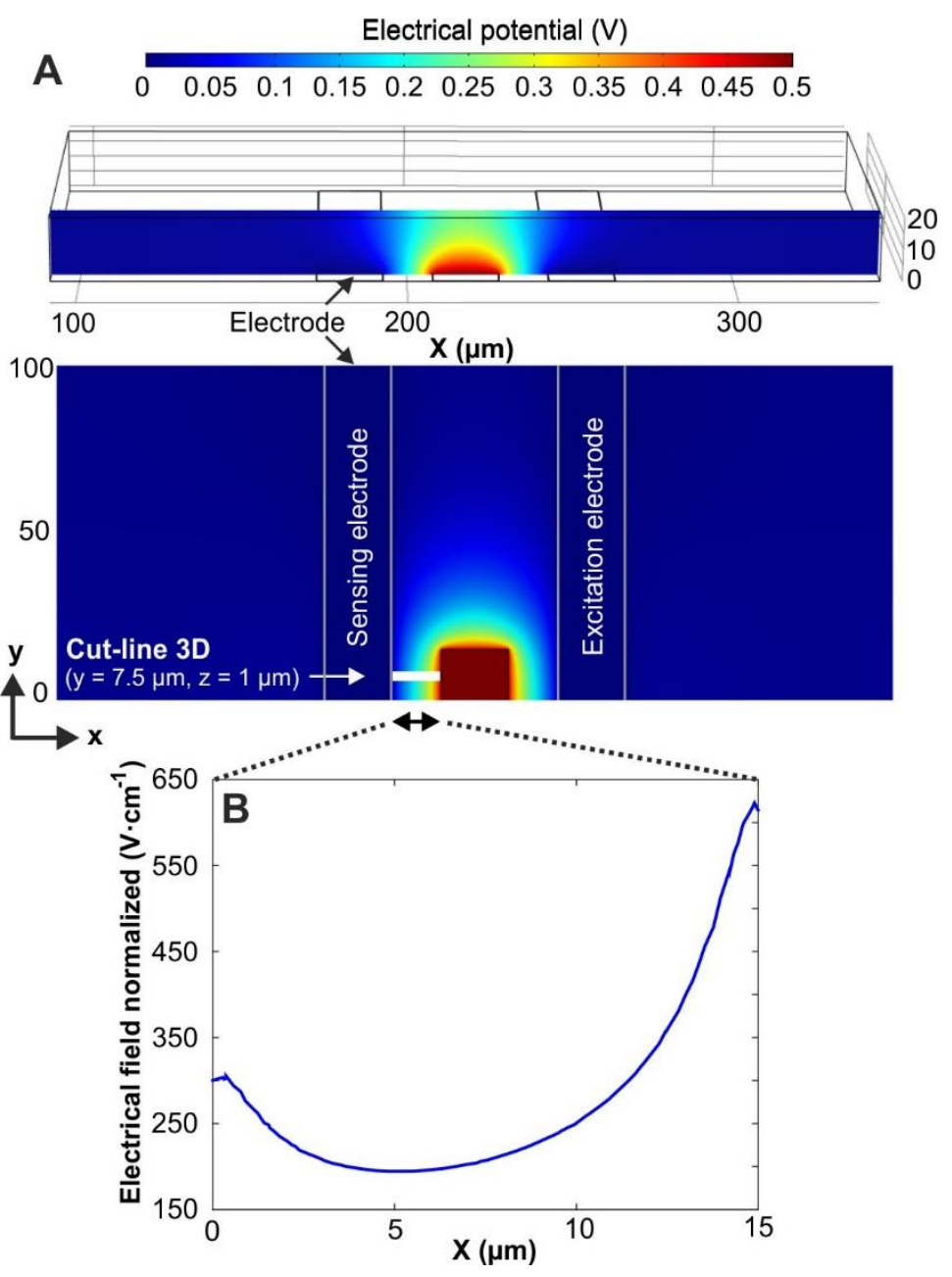

Fig. 5-11: COMSOL simulation (v4.4) of the electrical field within the top channel of the microfluidic device. This simulation is based on a 3D model (electrostatic), which described the electrical potential and normalized field strength under stationary conditions. A) The orthogonal and top view of the field potential showed non-uniform distribution due to the nonsymmetrical electrode geometry. B) A higher field strength was observed close to the excitation electrode compared to the sensing electrode when analysing the field strength in the middle of the electrode array $(y=7.5 \mu \mathrm{m})$ at a height $(z)$ of $1 \mu \mathrm{m}$. 
The observed increase in amplitude can be explained by position of the sperm head with respect to the electrodes and the resulting electrical field. As expected, the electrical field is non-uniform due to the planar electrode array geometry (Fig. 5-11A). When plotting the normalized electrical field in between the electrodes in the middle of the trapping site (Fig. 5-11B) at a height of $1 \mu \mathrm{m}$, the field strength close to the exciting electrode $(x=15 \mu \mathrm{m})$ was more than two times higher than the field strength close to the sensing electrode $(x=0 \mu \mathrm{m})$. As a consequence, sperm movement towards the exciting electrode will result in a higher change in impedance compared to movement towards the sensing electrode. This finding is consistent with the observed results. The hyperactivated spermatozoon was able to move closer to the exciting electrode, due to which the amplitude of the motilityrelated oscillation increased.

\subsection{Conclusions}

We characterized and developed a microfluidic platform for the entrapment of single spermatozoa and the analysis of single sperm motility. Most experiments present the analysis results of 1 or 2 sperm cells, therefore showing qualitative data. The sperm beat frequency was characterized optically and electrically and both characterizations showed identical results. Using electrical analysis, the effect of temperature alteration and caffeine exposure was observed on the sperm beat frequency. Furthermore, a relation was observed between the amplitude of the motility-related oscillation and the FBA. The ability to distinguish between nonhyperactivated and hyperactivated spermatozoa and to select accordingly, holds promise to improve sperm selection for ICSI treatments. Furthermore, both microfluidic and electrical analysis setups can be multiplexed in order achieve the required analysis throughput.

\subsection{Acknowledgements}

Financial support from the NWO - Netherlands Organization of Scientific Research (Spinoza Grant A. van den Berg, Veni L.I. Segerink), the scientific support of J.P. Frimat, C.C.L. Denoeud and J.T.W. Berendsen and the technical support of P.M. ter Braak and J. van Nieuwkasteele are gratefully acknowledged. We also thank the "KI Twenthe" for the kind supply of boar semen samples. 


\subsection{References}

1. Kupka, M.S., et al., Assisted reproductive technology in Europe, 2010: results generated from European registers by ESHRE. Hum Reprod, 2014. 29(10): p. 2099-113.

2. Nyboe Andersen, A., Gianaroli, L., Nygren, K.G. , et al., Assisted reproductive technology in Europe, 2000. Results generated from European registers by ESHRE. Hum Reprod, 2004. 19(3): p. 490-503.

3. Boulet, S.L., et al., TRends in use of and reproductive outcomes associated with intracytoplasmic sperm injection. JAMA, 2015. 313(3): p. 255-63.

4. Benchaib, M., et al., Sperm DNA fragmentation decreases the pregnancy rate in an assisted reproductive technique. Hum Reprod, 2003. 18(5): p. 1023-8.

5. Mokánszki, A., et al., Is sperm hyaluronic acid binding ability predictive for clinical success of intracytoplasmic sperm injection: PICSI vs. ICSI? Syst Biol Reprod Mec, 2014. 60(6): p. 348-54.

6. Pregl Breznik, B., B. Kovačič, and V. Vlaisavljević, Are sperm DNA fragmentation, hyperactivation, and hyaluronan-binding ability predictive for fertilization and embryo development in in vitro fertilization and intracytoplasmic sperm injection? Fertil Steril, 2013. 99(5): p. 1233-41.

7. Fuhr, G., et al., High-frequency electric field trapping of individual human spermatozoa. Hum Reprod, 1998. 13(1): p. 136-41.

8. Nascimento, J.L., et al., Analysis of sperm motility using optical tweezers. J Biomed Opt, 2006. 11(4): p. 044001-1-8.

9. Chen, Y.A., et al., Direct characterization of motion-dependent parameters of sperm in a microfluidic device: proof of principle. Clin Chem, 2013. 59(3): p. 493-501.

10. Frimat, J.P., et al., Make it spin: individual trapping of sperm for analysis and recovery using micro-contact printing. Lab Chip, 2014. 14(15): p. 263541.

11. de Wagenaar, B., et al., Microfluidic single sperm entrapment and analysis. Lab Chip, 2015. 15(5): p. 1294-1301.

12. Heileman, K., J. Daoud, and M. Tabrizian, Dielectric spectroscopy as a viable biosensing tool for cell and tissue characterization and analysis. Biosens Bioelectron, 2013. 49(0): p. 348-59.

13. Sun, T. and H. Morgan, Single-cell microfluidic impedance cytometry: $a$ review. Microfluid Nanofluid, 2010. 8(4): p. 423-43.

14. Haandbaek, N., et al., Characterization of subcellular morphology of single yeast cells using high frequency microfluidic impedance cytometer. Lab Chip, 2014. 14(2): p. 369-77. 
15. Holmes, D., et al., Leukocyte analysis and differentiation using high speed microfluidic single cell impedance cytometry. Lab Chip, 2009. 9(20): p. 28819.

16. Kirkegaard, J., et al., Study of Paclitaxel-Treated HeLa Cells by Differential Electrical Impedance Flow Cytometry. Biosensors, 2014. 4(3): p. 257-72.

17. David, F., et al., Viability and membrane potential analysis of Bacillus megaterium cells by impedance flow cytometry. Biotechnol Bioeng, 2012. 109(2): p. 483-92.

18. Jackson, R.E., et al., Effects of semen storage and separation techniques on sperm DNA fragmentation. Fertility and Sterility, 2010. 94(7): p. 2626-2630.

19. Nosrati, R., et al., Rapid selection of sperm with high DNA integrity. Lab Chip, 2014. 14(6): p. 1142-50.

20. Zhang, B., T.L. Yin, and J. Yang, A novel microfluidic device for selecting human sperm to increase the proportion of morphologically normal, motile sperm with uncompromised DNA integrity. Anal Method, 2015. 7: 5981-8.

21. Chan, P.J., et al., A simple comet assay for archived sperm correlates DNA fragmentation to reduced hyperactivation and penetration of zona-free hamster oocytes. Fertil Steril, 2001. 75(1): p. 186-92.

22. Tasoglu, S., et al., Exhaustion of Racing Sperm in Nature-Mimicking Microfluidic Channels During Sorting. Small, 2013. 9(20): p. 3374-84.

23. Cho, B.S., et al., Passively Driven Integrated Microfluidic System for Separation of Motile Sperm. Anal Chem, 2003. 75(7): p. 1671-5.

24. Seo, D.-b., et al., Development of sorting, aligning, and orienting motile sperm using microfluidic device operated by hydrostatic pressure. Microfluid Nanofluid, 2007. 3(5): p. 561-70.

25. Saravia, F., et al., Differences in boar sperm head shape and dimensions recorded by computer-assisted sperm morphometry are not related to chromatin integrity. Theriogenology, 2007. 68(2): p. 196-203.

26. Petrunkina, A.M., et al., Requirement for an intact cytoskeleton for volume regulation in boar spermatozoa. Reproduction, 2004. 127(1): p. 105-15.

27. Kondracki, S., et al., Comparative analysis of Duroc and Pietrain boar sperm morphology. Acta Veterinaria Brno, 2012. 81(2): p. 195-9.

28. Yamaguchi, S., et al., Effects of caffeine on sperm characteristics after thawing and inflammatory response in the uterus after artificial insemination with frozen-thawed boar semen. Theriogenology, 2013. 79(1): p. 87-93.

29. Garbers, D.L., et al., Stimulation and Maintenance of Ejaculated Bovine Spermatozoan Respiration and Motility by Caffeine. Biol Reprod, 1971.5(3): p. 336-9. 
30. Qiu, T., et al. A microfluidic "treadmill" for sperm selective trapping according to motility classification. in Solid-State Sensors, Actuators and Microsystems Conference (transducers), 2011 16th International. 2011.

31. Seo, D.B., et al., Development of sorting, aligning, and orienting motile sperm using microfluidic device operated by hydrostatic pressure. Microfluid Nanofluid, 2007. 3(5): p. 561-70.

32. Lopez-Garcia, M.D.C., et al., Sperm motion in a microfluidic fertilization device. Biomed Microdevices, 2008. 10(5): p. 709-18.

33. Mortimer, D., Practical Laboratory Andrology. 1994: Oxford University Press.

34. McQueen, C.A., Comprehensive toxicology. Second edition ed. 2010: Elsevier Ltd.

35. Gatti, J.-L., et al., External ionic conditions, internal $\mathrm{pH}$ and motility of ram and boar spermatozoa. J Reprod Fertil, 1993. 98(2): p. 439-49.

36. Satake, N., et al., Sperm selection and competition in pigs may be mediated by the differential motility activation and suppression of sperm subpopulations within the oviduct. J Exp Biol, 2006. 209(8): p. 1560-72.

37. Barboni, B., M. Mattioli, and E. Seren, Influence of progesterone on boar sperm capacitation. Journal of Endocrinol, 1995. 144(1): p. 13-8.

38. Peláez, J., et al., In vitro Evaluation of the Quality and Fertilizing Capacity of Boar Semen Frozen in $0.25 \mathrm{~mL}$ straws. Reprod Domest Anim, 2006. 41(2): p. 153-61.

39. Schmidt, H. and G. Kamp, Induced hyperactivity in boar spermatozoa and its evaluation by computer-assisted sperm analysis. Reproduction, 2004. 128(2): p. 171-9.

40. Jensen, T.K., et al., Caffeine Intake and Semen Quality in a Population of 2,554 Young Danish Men. Am J Epidemiol, 2010. 171(8): 883-91.

41. Mortimer, S.T., et al., Quantitative observations of flagellar motility of capacitating human spermatozoa. Hum Reprod, 1997. 12(5): p. 1006-12.

42. Schmidt, H. and U. Kamp, Induced hyperactivity in boar spermatozoa and its evaluation by computer-assisted sperm analysis. Reproduction, 2004. 128(2): p. 171-9. 


\section{Towards microfluidic sperm refinement: impedance- based analysis and sorting of sperm cells}

The use of high quality semen for artificial insemination in the livestock industry is essential for successful outcome. Insemination using semen with a high number of sperm cells containing morphological defects, such as cytoplasmic droplets, has a negative impact on fertilization outcome. Therefore, semen with a high number of these abnormal cells is discarded in order to maintain high fertilization potential, resulting in the loss of a large number of morphologically normal sperm cells. No techniques are available to extract morphologically normal sperm cells from rejected samples. We aim to develop a microfluidic setup which is able to detect and sort morphologically normal sperm cells label-free and non-invasively to retrieve these cells from otherwise discarded samples.

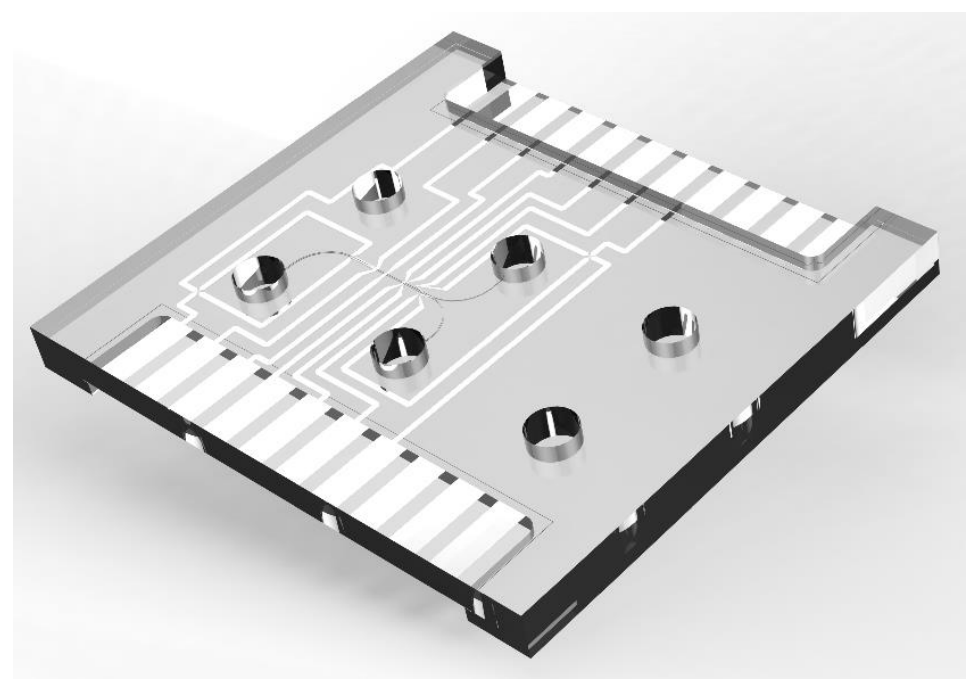

Graphical illustration of the microfluidic device for sperm analysis and sorting (SolidWorks 3D model, S. Dekker)

Adapted from: de Wagenaar, B., Dekker, S., Bomer, J.G., van Nieuwkasteele, J.W. Olthuis, O., van den Berg, A., Segerink, L.I., Towards microfluidic sperm refinement: impedancebased analysis and sorting of sperm cells. (submitted) 


\subsection{Introduction}

Al is a well-established technique in the animal industry for livestock production. Selection of sperm samples for Al is based on sperm concentration, cell motility and morphology [1]. All factors have shown impact on the success rate of fertilization and the abundance of offspring [1-4]. Therefore, insemination stations live up to high standards to supply high quality semen samples to ensure high probability of fertilization after Al. Criteria for semen sample rejection are for example reduced sperm cell motility (motility $<70 \%$ ) and/or high numbers of morphologically abnormal sperm cells (> $20 \%$ abnormal cells) $[5,6]$.

A frequently occurring sperm anomaly is the presence of a cytoplasmic droplet on the sperm flagellum. This droplet is part of the cytoplasm of the spermatids, which was not removed from the flagellum at the end of spermatogenesis [7]. Although the effect of residual cytoplasm retention on human infertility is a controversial subject in the clinic [8], several sources show a significant effect of droplet content on infertility in domestic species $[8,9]$. In practice, semen samples containing over $15-20 \%$ of cells with cytoplasmic droplets are withheld from Al $[5,6,9]$. In this process, a high number of healthy, morphologically normal sperm cells are discarded. Unfortunately, routine sperm refinement techniques such as sperm density centrifugation and sperm swim-up are not suitable for recovering these sperm cells for Al purposes.

A potential approach to obtain these healthy and morphologically normal sperm cells from rejected samples is the use of microfluidic technology. Microfluidic systems have been used for the manipulation, analysis and enrichment of viable, motile sperm cells [10-14]. However, none of these systems is currently capable of performing sperm analysis and selection based on cell morphology (on the single cell level).

A possible method to distinguish morphologically normal sperm cells from cytoplasmic droplet-containing cells is impedance cytometry. Numerous reports show the use of impedance cytometry to perform high-throughput analysis of biological species including bacteria, yeast, cancer cells and various blood cell types, investigating cell properties such as cell size, membrane integrity and cytoplasm conductivity [15-18]. Previous work in our group showed the potential to perform impedance cytometry on sperm cells, specifically [19].

Besides high-throughput analysis capability, the microfluidic system must include an actively-controlled sorting mechanism to separate abnormal sperm cells. Active 
size sorting of single plastic beads using dielectrophoresis (DEP) based on impedance analysis has been shown before [20]. Many have reported upon the use of DEP as a versatile tool for particle and cell trapping, manipulation and sorting [21-23]. Furthermore, DEP has been used to manipulate and analyse sperm cells $[13,24]$. Therefore, the combination of impedance detection and DEP sorting is an interesting approach towards sorting of sperm cells based on cytoplasmic droplet content.

\subsection{Materials \& Methods}

\subsubsection{Chip fabrication}

Microfluidic chips (Fig. 6-1) were fabricated using routine photolithography, wet etch, sputter and bonding techniques. The fabrication process is illustrated in Fig. 6-2 and described in detail in Appendix A.

After cleaning two borofloat glass wafers (BF33, $100 \mathrm{~mm}$ diameter, 500 and 1100 $\mu \mathrm{m}$ thick), microelectrodes were fabricated after resist deposition, exposure and developing, BHF wet-etching, deposition of titanium/platinum layers (layer thickness 30 and $120 \mathrm{~nm}$, respectively) and resist lift-off. Subsequently, inserts for fluidic and electric connections were powderblasted through both wafers (particle size $29 \mu \mathrm{m}$ ).

A

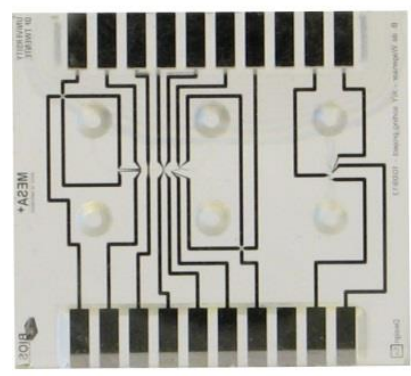

B

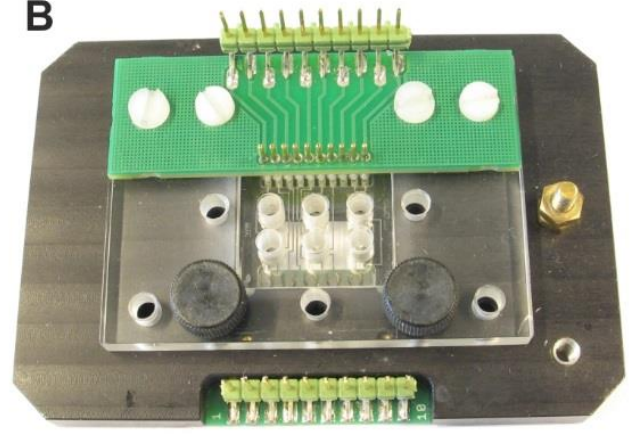

Fig. 6-1: A) Microfluidic device and B) chip holder. Push-pin electrodes are used to form electrical connections to the top and bottom electrodes on the chip. NanoPorts are used as fluidic connectors between $360 \mu \mathrm{m}$ fused silica capillaries and the fluidic channels on-chip. The bottom part of the chip holder is made of anodized aluminium; the top part is made of transparent polycarbonate to allow visualization of the sperm cells using phase contrast microscopy. 
$500 \mu \mathrm{m}$ BF33

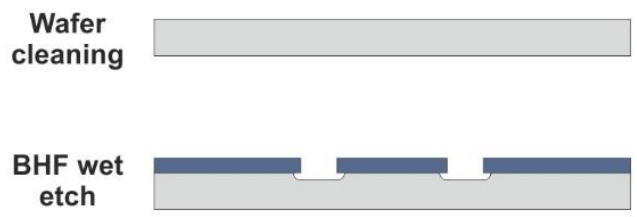

Sputtering

\& lift-off

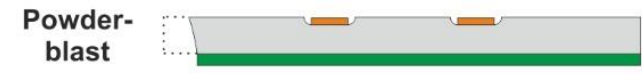

Foil lamination

Alignment \& bonding

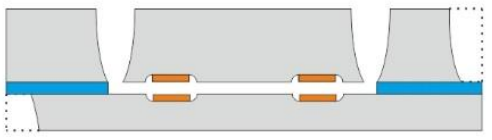

$1100 \mu \mathrm{m}$ BF33
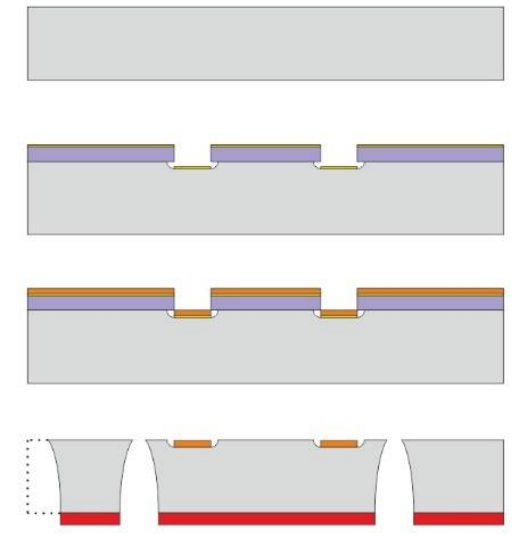

Resist mask: electrodes $\mathbf{5 0 0} \mu \mathrm{m}$

Resist mask: electrodes $1100 \mu \mathrm{m}$

Resist mask: powderblast $500 \mu \mathrm{m}$

Resist mask: powderblast $1100 \mu \mathrm{m}$

Resist mask: PerMX foil $\mathbf{5 0 0} \mu \mathrm{m}$

Fig. 6-2: Schematic illustration of device fabrication. After cleaning and BHF wet etching, tantalum (30 nm) and platinum (120 nm) were sputtered on the borofloat wafers (BF33). After powderblasting the entries for the fluidic and electric connections, a $20 \mu \mathrm{m}$ polymer film (PerMX3020) was laminated on the $500 \mu \mathrm{m}$ wafer. After development of the microfluidic channel, the wafers were aligned and bonded together.

After cleaning the wafers using ultrasound and $\mathrm{HNO}_{3}$, a layer of foil $(20 \mu \mathrm{m}$, PerMX3020, Dupont) was laminated (foil laminator) on the $500 \mu \mathrm{m}$ wafers at $80^{\circ} \mathrm{C}$ using a roller speed of $300 \mathrm{~mm} \cdot \mathrm{min}^{-1}$.

After lamination, the wafers were pre-baked $\left(5 \mathrm{~min}\right.$ at $50{ }^{\circ} \mathrm{C}, 5 \mathrm{~min}$ at $65^{\circ} \mathrm{C}$ and 10 min at $85^{\circ} \mathrm{C}$ ) to improve adhesion of the foil to the glass. Exposure of the layer was performed by illumination through the mask using a $12 \mathrm{~mW} \cdot \mathrm{cm}^{-2}$ UV source (EVG 620) for $40 \mathrm{~s}$ using an $8 \times 5 \mathrm{~s}$ interval exposure to prevent gas bubble formation in the polymer layer. Subsequently, a post-exposure bake was performed ( $5 \mathrm{~min}$ at 50 ${ }^{\circ} \mathrm{C}, 5 \mathrm{~min}$ at $65^{\circ} \mathrm{C}$ and $10 \mathrm{~min}$ at $85^{\circ} \mathrm{C}$ ). The polymer layer was developed with RER600 (PGMEA, Arch Chemicals, Inc.) using a spin-coater (2000 rpm, 3 runs of $15 \mathrm{~s}$ ). 
After aligning the $500 \mu \mathrm{m}$ wafers with respect to the $1100 \mu \mathrm{m}$ wafers in a bond chuck, they were bonded together $\left(30 \mathrm{~min}, 1000 \mathrm{~N}\right.$ piston pressure, $0 \mathrm{~V}, 10^{-1} \mathrm{mbar}$, $100{ }^{\circ} \mathrm{C}$ ) in an anodic bonder (EV-501). Subsequently, the wafer stack was hardbaked $\left(1 \mathrm{~h}, 1-2\right.$ ton, $150^{\circ} \mathrm{C}$ ) in a heated press (Carver). After dicing (Dicing saw Disco DAD 321), the chips were ready to use.

Two different chip designs were used in the reported experiments. For cytoplasmic droplet detection experiments, sperm cells were flown through $20 \mu \mathrm{m}$ high and 20 $\mu \mathrm{m}$ wide microfluidic channel (Fig. 6-5A). Two electrode pairs were used to measure the impedance differentially, in which the width of the electrodes was $10 \mu \mathrm{m}$ and distance between the electrode pairs was $20 \mu \mathrm{m}$. For cell sorting experiments, the microfluidic chip consisted of a $20 \mu \mathrm{m}$ high and $100 \mu \mathrm{m}$ wide channel containing integrated electrodes for impedance detection and DEP focusing and sorting (Fig. 6-13A).

\subsubsection{Sample \& chip preparation}

Fresh boar semen was obtained from a local artificial insemination centre ("KI Twenthe", The Netherlands) at a concentration of $20 \cdot 10^{6}$ cells $\cdot \mathrm{mL}^{-1}$. The samples were diluted with Beltsville Thawing Solution (BTS, Solusem, Aim Worldwide) to a concentration of $2 \cdot 10^{6}$ cells $\cdot \mathrm{mL}^{-1}$.

Before each experiment, microfluidic channels were coated with poly(L-lysine)grafted-poly(ethylene glycol) (PLL(20)-g[3.5]-PEG(2), SuSoS) to prevent cell adhesion. PLL-g-PEG was rinsed through the channels at a concentration of 100 $\mu \mathrm{g} \cdot \mathrm{mL}^{-1}$ in DI water for at least $15 \mathrm{~min}$ at a flow rate of $0.5-1 \mu \mathrm{L} \cdot \mathrm{min}^{-1}$ using a syringe pump (neMESYS, Cetoni GmbH) equipped with a Hamilton gastight syringe (1710N). BTS solution was rinsed for at least $15 \mathrm{~min}$ at a flow rate of $0.5-1 \mu \mathrm{L} \cdot \mathrm{min}^{-1}$ to remove remaining coating solution. Subsequently, sperm solution was flushed through the channel at a flow rate of $0.5-1 \mu \mathrm{L} \cdot \mathrm{min}^{-1}$. Upon visualization in the microfluidic channel, the flow rate was changed to $0.02-0.025 \mu \mathrm{L} \cdot \mathrm{min}^{-1}$ before impedance acquisition.

\subsubsection{Impedance detection $\&$ analysis}

Impedance was recorded using an impedance spectroscope (HF2IS, Zurich Instruments, Zurich, Switzerland) equipped with a preamplifier (HF2TA, Zurich Instruments), illustrated in Fig. 6-3. Two different modes of operation were used in the experiments. 


\section{A Differential mode (cytoplasmic droplet detection)}

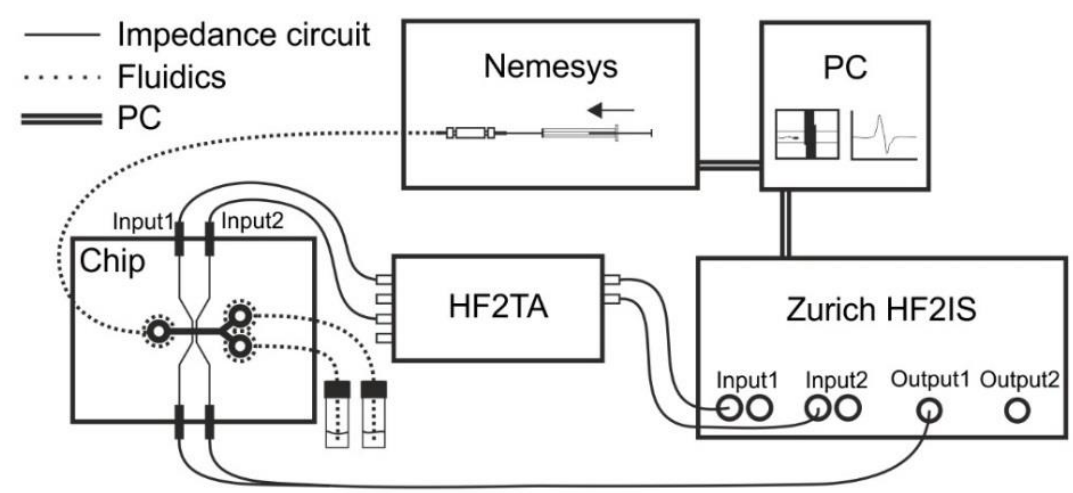

B Non-differential mode (cell sorting)

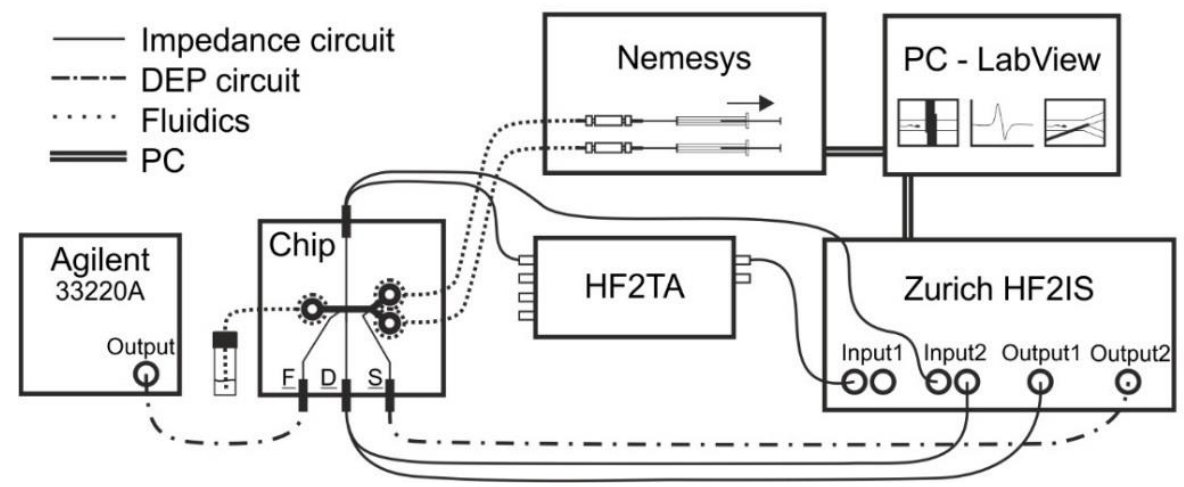

Fig. 6-3: A) For cytoplasmic droplet detection experiments, the impedance was recorded differentially using two closely spaced electrode pairs, which were connected to input 1 and 2 of the Zurich impedance spectroscope (HF2IS) via an HF2TA current preamplifier. Output 1 of the HF2IS was used to apply a sinusoidal excitation to the on-chip electrodes (in both setups). Fluid flow was controlled using a neMESYS syringe pump. B) For cell sorting experiments, the impedance was recorded using a single electrode pair in 4-point configuration. The current was recorded at input 1 while recording the voltage at input 2 , simultaneously. An Agilent waveform generator was used to apply a $10 \mathrm{MHz}$ signal to the focusing electrodes. Finally, output 2 of the HF2IS was used to selectively excite the sorting electrodes. Data acquisition and active control of cell sorting were performed by LabVIEW (PC). 
For cytoplasmic droplet detection experiments, the impedance was recorded in differential mode (Fig. 6-3A) using the chip design illustrated in Fig. 6-5A. In this mode, an AC signal with an amplitude of $0.5 \mathrm{~V}$ and a frequency of $1.3 \mathrm{MHz}$ was generated on output 1 and applied to the differential electrode pair of the device under test (DUT). The two corresponding electrodes of the differential electrode pair were connected to input 1 and input 2 of the impedance spectroscope via two separate current amplifying channels of the current preamplifier (10k amplification factor). The impedance was recorded using a bandwidth of $200 \mathrm{~Hz}$ and a sampling frequency of $3598 \mathrm{~Hz}$. For cell sorting experiments, the impedance was measured in non-differential mode (Fig. 6-3B) using the chip design shown in Fig. 6-13A. In this mode, 4-point measurements were performed. The current was amplified ( $10 \mathrm{k}$ amplification factor) using channel 1 of the preamplifier connected to input 1 of the impedance spectroscope. The voltage was measured differentially at input 2 . In this mode, impedance was recorded using a $1 \mathrm{MHz}$ sinusoidal excitation with an amplitude of $0.5 \mathrm{~V}$. In this mode of operation, impedance was recorded using a bandwidth of $200 \mathrm{~Hz}$ and a sampling frequency of $899 \mathrm{~Hz}$ unless mentioned otherwise.

Recorded impedance data was imported and processed in Matlab (R2014a, MathWorks). For measurements in differential mode, the absolute impedance data from input 2 was subtracted from signal 1 to obtain the differential signal. Subsequently, baseline correction and peak detection were performed. In nondifferential mode, drift and offset were removed by baseline correction before peak detection.

\subsubsection{Sperm cell focusing \& sorting by DEP}

The sperm cell orientation and location within a $100 \mu \mathrm{m}$ channel (Fig. 6-13A) was manipulated by applying DEP using two top-down electrode pairs. Cell focusing was performed by applying a $10 \mathrm{MHz} 3 \mathrm{~V}$ sinusoidal excitation on the focusing electrodes (Agilent 33220A, Agilent Technologies, Inc.). Similarly, cell sorting was performed using $15 \mathrm{MHz} 2 \mathrm{~V}$ excitation using output 2 of the impedance spectroscope.

\subsubsection{Video acquisition \& optical analysis}

Optical data was recorded using a Nikon TE2000-U microscope equipped with a 10x phase contrast objective (and Ph1 condenser annulus) and a Basler acA780-75 camera at $25 \mathrm{fps}$. Sperm tracking was performed using the "motion-based multiple object tracking" function of the computer vision system toolbox in Matlab. This 
function processes every frame one by one and detects objects by comparison to a static background. These objects were tracked over time and assigned to object trajectories. This readily available function in Matlab was adapted to allow storage of objects' time data and location.

\subsubsection{Integrated data acquisition \& DEP sorting using LabVIEW}

Acquisition of impedance data and actively controlled DEP sorting was performed by a custom-built LabVIEW program. This program consists of readily available virtual instrument (VI) drivers for all involved equipment (Zurich HF2IS, Basler Camera acA780-75gc, neMESYS syringe pump) and a control algorithm, which processes measurement data and controls DEP excitation. When operational, the control program monitors the impedance over time. Upon particle or cell passing, the change in impedance was recorded and matched to a predefined template (Fig. 6-4). When the detection criterion, which is based on the quality of the fit and the optimal scaling factor, crossed the threshold value, a positive match was determined [25]. In case of a positive match, a Gaussian distribution is fitted to the recorded impedance change after which a derivative is calculated. This derivative is used to estimate the velocity of the particle or cell and the estimated time $\left(t_{x}\right)$ of arrival (ETA) at the sorting electrodes. Subsequently, the total change in impedance is matched to the window of interest (WOI). When the impedance change fits within the WOI, the DEP sorting electrodes are activated at interval $t_{0} \ldots t_{1}$ to sort the particle or cell in the top channel. When the impedance change does not match the WOI, the DEP sorting electrodes are inactive at the interval $t_{0} \ldots t_{1}$.

\subsubsection{Simulation}

The electrical response of the microfluidic setup was investigated by constructing a numerical model of the circuit in Matlab. This model is well described in literature [26] and describes the simplified electrical circuit model (ECM) for a single-shelled spheroid in suspension. In simulations, a parallel electrode configuration was modelled without field fringing at the electrode edges. Sperm cells were modelled as single-shelled spheroids with similar cell volume $\left(1.25 \cdot 10^{-17} \mathrm{~m}^{3}\right)$ [27].

The absolute impedance response of the microfluidic setup was simulated as a function of frequency $(200 \mathrm{~Hz}-2 \mathrm{MHz})$. This simulation was compared to a real measurement, in which the absolute impedance was recorded in identical frequency range. Furthermore, a sperm cell was simulated in between the electrodes to investigate the expected impedance change. Simulation parameters, which are used for the described simulations, are reported in Fig. 6-5. 


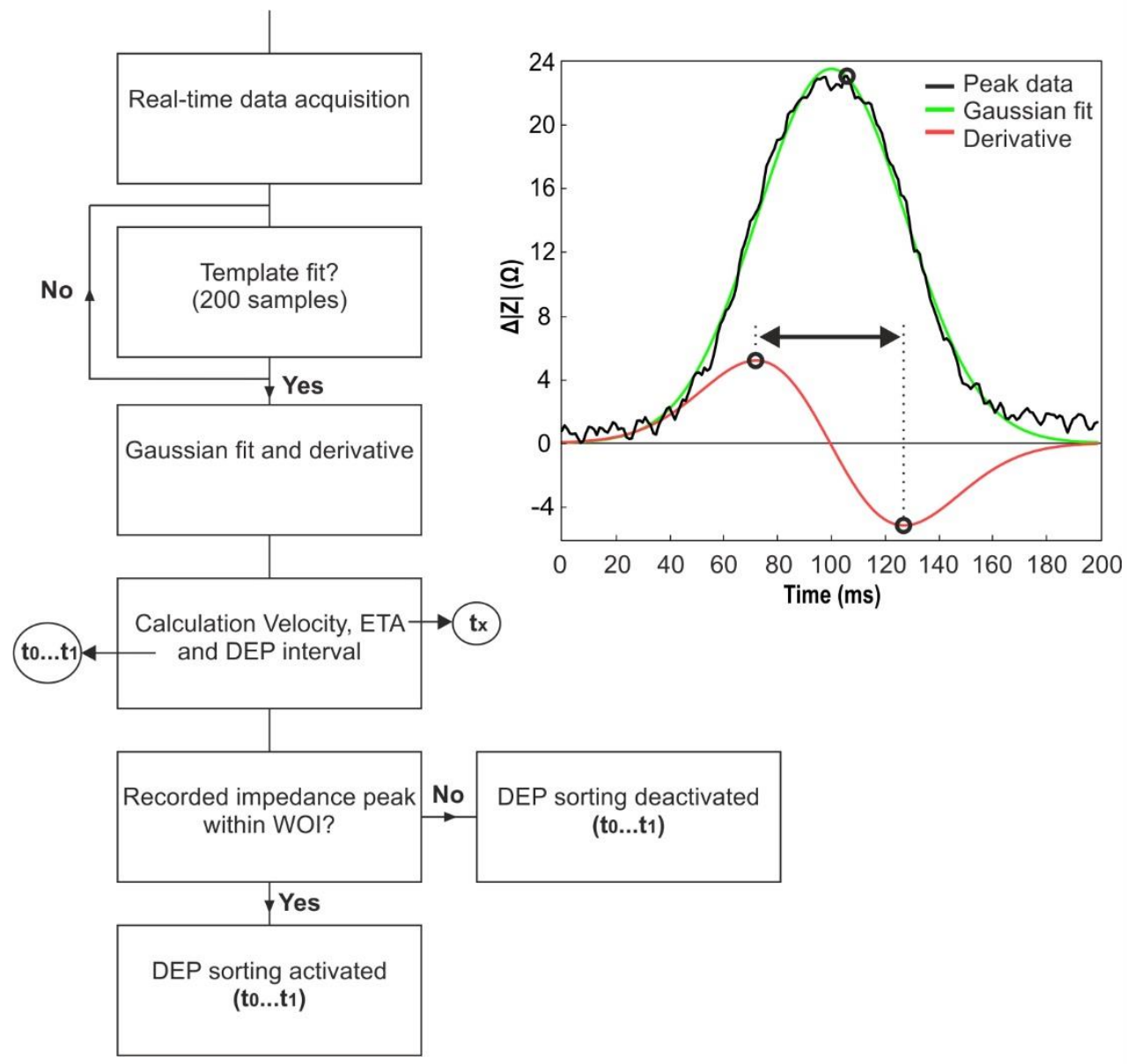

Fig. 6-4: Flowchart of LabVIEW controlled peak sorting. After fitting the data to a predefined peak template, a Gaussian distribution is fitted to the detected peak after which the velocity is calculated. Subsequently, the estimated time of arrival and the window of DEP activation or deactivation $\left(t_{0} \ldots t_{1}\right)$ is calculated.

\subsection{Results \& discussion}

\subsubsection{Simulation}

Impedance spectroscopy is a commonly used tool for label-free analysis of adherent cells or cells in suspension. This technique has been used extensively to investigate the dielectric properties of cells in microfluidic systems. 
Constructing an ECM is a simple way to gain insight into the electrical response of the microfluidic setup (Fig. 6-5A). The capacitive properties of the microelectrode setup are predominantly determined by the electrode/electrolyte interface $\left(C_{D L}\right)$ and the electrolyte $\left(C_{e l}\right)$. The resistive response is influenced by the lead wires $\left(R_{\text {lead }}\right)$ and the conductivity of the electrolyte $\left(R_{e l}\right)$. When a spermatozoon is introduced between the microelectrodes, the capacitive and resistive properties will be altered by the cell membrane $\left(C_{\text {mem }}\right)$ and the cell's cytoplasm $\left(R_{i}\right)$, respectively. The simulation is based on the equivalent circuitry of a parallel electrode pair in the absence of a sperm cell as illustrated in (Fig. 6-5B). The simulation of the electrical response showed an expected effect of the electrodeelectrolyte interface on the absolute impedance (Fig. 6-5C).

Due to a small electrode surface area and a small $C_{D L}$ correspondingly, the impedance continuously decreased over a broad frequency range where $C_{D L}$ was dominant. At a frequency of approximately $1.3 \mathrm{MHz}$ a resistive plateau was formed where $R_{e l}$ was dominant. A frequency sweep of a single electrode pair in the absence of a sperm cell showed similar behaviour compared to the simulation, indicating that a measurement frequency of $1.3 \mathrm{MHz}$ is an appropriate choice for sperm impedance analysis using this setup. At this frequency, simulation showed an impedance increase of $299 \Omega$ when modelling a sperm cell in between the electrodes using conditions as reported in Fig. 6-5.

\subsubsection{Electrical analysis of cell orientation and morphology}

Impedance analysis of sperm cells was performed by flowing cells through a $20 \mu \mathrm{m}$ high, $20 \mu \mathrm{m}$ wide channel restriction (Fig. 6-5A) at a flow rate between 0.013 and $0.02 \mu \mathrm{L} \cdot \mathrm{min}^{-1}$. In this constriction area, the impedance was recorded differentially using two electrode pairs with an electrode width of $10 \mu \mathrm{m}$ and an electrode separation of $20 \mu \mathrm{m}$ as illustrated in Fig. 6-3A. After calculating the difference between the electrical responses of both electrode pairs, baseline correction and peak detection were performed. The resulting peak heights showed a wide distribution in impedance, ranging from values between 200 and $1500 \Omega$ (Fig. 6-6). This broad distribution is caused by the effects of cell location and tilting on the impedance. Especially cell tilting showed a big effect on the impedance. When the sperm cell is aligned with the electrodes, e.g. the flat side of the sperm cell runs parallel with respect to the electrode surface (high cross-sectional area), a high impedance change is recorded (optically verified, results not shown). However, when a sperm cell is rotated 90 degrees over is longitudinal axis and only a small part of the sperm is exposed (small cross-sectional area), the recorded impedance 

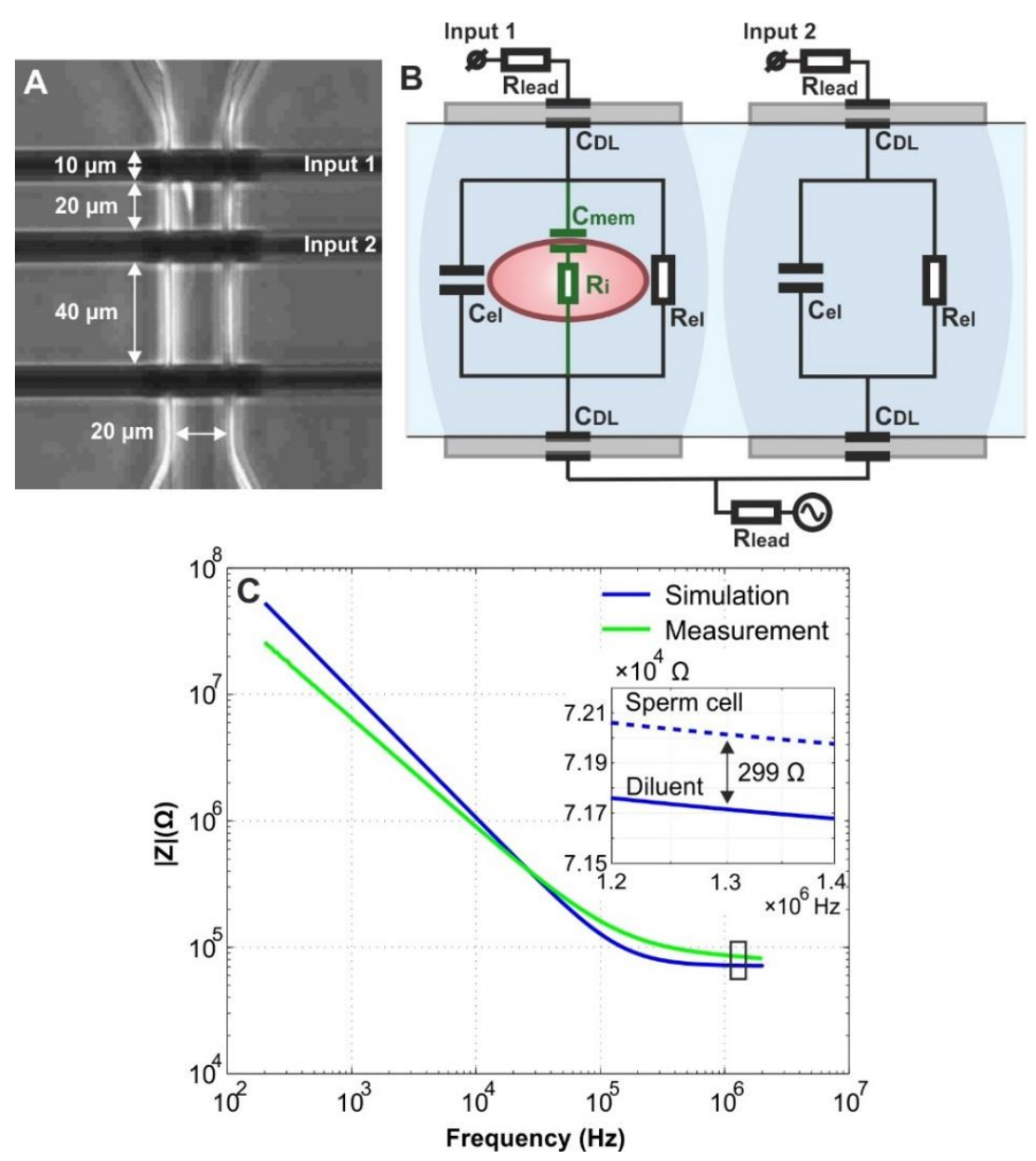

Fig. 6-5: A) Microfluidic chip consisting of two electrode pairs for differential impedance analysis. B) Electric circuit model (ECM) of the measurement setup. Without a cell in between the electrodes (input 2), the setup is described by an electrode-electrolyte interface $\left(C_{D L}\right)$, electrolyte $\left(R_{e l} \& C_{e l}\right)$ and the wire resistance $\left(R_{\text {lead }}\right)$. A passing sperm cell adds a cell membrane capacitance $\left(C_{m e m}\right)$ and cytoplasm resistance $\left(R_{i}\right)$ to the ECM, considering Foster and Schwan's simplified ECM for a single-shelled spheroid in suspension (input 1). C) Simulated and measured impedance versus frequency using the following parameters: $s=l=20 \mu \mathrm{m}, w=10 \mu \mathrm{m}, A=2 \cdot 10^{-12} \mathrm{~m}^{2}, V_{\text {cell }}=12.5 \mathrm{fL}, r=1.44 \mu \mathrm{m}, d_{m}$ $=5 \cdot 10^{-9} \mathrm{~m}, \sigma_{e l}=1.4 \mathrm{~S} \cdot \mathrm{m}^{-1}, \sigma_{i}=0.4 \mathrm{~S} \cdot \mathrm{m}^{-1}, \sigma_{m e m}=10^{-8} \mathrm{~S} \cdot \mathrm{m}^{-1} \cdot \varepsilon_{e l}=80, \varepsilon_{i}=$ $60, \varepsilon_{\text {mem }}=11.3, C_{d}=20 \mu \mathrm{F} \cdot \mathrm{cm}^{-2}, R_{\text {lead }}=0 \Omega$. 
changes are small (around $200 \Omega$ ). In the simulation, a sperm cell is modelled as a spheroid with similar volume, due to which its cross-sectional area is much smaller compared to a sperm cell that is aligned with the electrodes. Therefore, the simulated impedance is more comparable to a sperm cell, which passed the electrodes in tilted orientation.

Due to the effects of location and orientation, the absolute change in impedance is not a suitable parameter to characterize morphological differences. A different approach is the analysis of the peak shape over time. A sperm cell is shaped as a triaxial ellipsoid with a head length of 8-9 $\mu \mathrm{m}$, head width of 4-5 $\mu \mathrm{m}$, head thickness of $<0.5 \mu \mathrm{m}$ and an average tail length $40-50 \mu \mathrm{m}$ ) $[28,29]$. Its total length is larger than the channel width and height and the width of the microelectrodes. When a sperm cell is flown through this microchannel, the cell will align itself over its longitudinal axis with respect to the channel wall. Consequently, the distinct parts of the sperm cell (head, midpiece and flagellum) will pass the electrical field between the microelectrodes at different points in time and will affect the recorded impedance, accordingly. As a result, the peak shape may contain information about the cell orientation (head-first or tail-first) and its morphology.

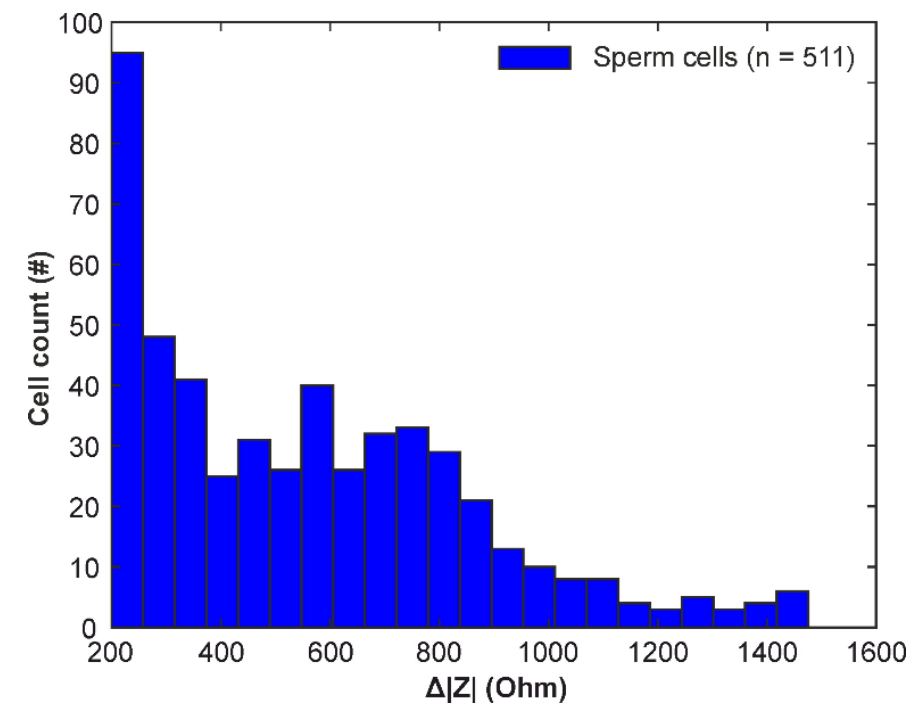

Fig. 6-6: Impedance change distribution of sperm cells measured in a $20 \mu \mathrm{m}$ wide and $20 \mu \mathrm{m}$ high channel with 10 $\mu \mathrm{m}$ wide electrodes. A broad distribution in recorded impedance was observed ranging between 200 and $1500 \Omega$. 
To test this hypothesis, the impedance peak shape of passing sperm cells was investigated (using an electrode separation of $20 \mu \mathrm{m}$ ). The measured curves showed a positive and negative peak (Fig. 6-7), corresponding to sperm passing through the first and second electrode pair, respectively. At zero-crossing, the recorded impedance at input 1 and 2 is equal, at which point the sperm head is positioned in between the two electrode pairs, approximately.

The curves showed a clear effect of the cell orientation on the peak shape. When a sperm cell passed the two electrode pairs head-first (Fig. 6-7A), the differential impedance change showed a positive peak followed by a negative peak with signal tailing before returning to a steady baseline level. This tailing effect was caused by the presence of the sperm flagellum in between the electrodes. In tail-first orientation, this tailing effect was observed before the sperm head arrived at the first electrode pair (Fig. 6-7B).

Furthermore, information about droplet content could be extracted from the peak data. A clear example is shown in Fig. 6-7C, in which a bump in the signal was observed. This bump in impedance was located in between the negative peak (sperm head) and the residual impedance change (sperm flagellum). This bump was caused by the presence of a distal cytoplasmic droplet on the sperm tail.
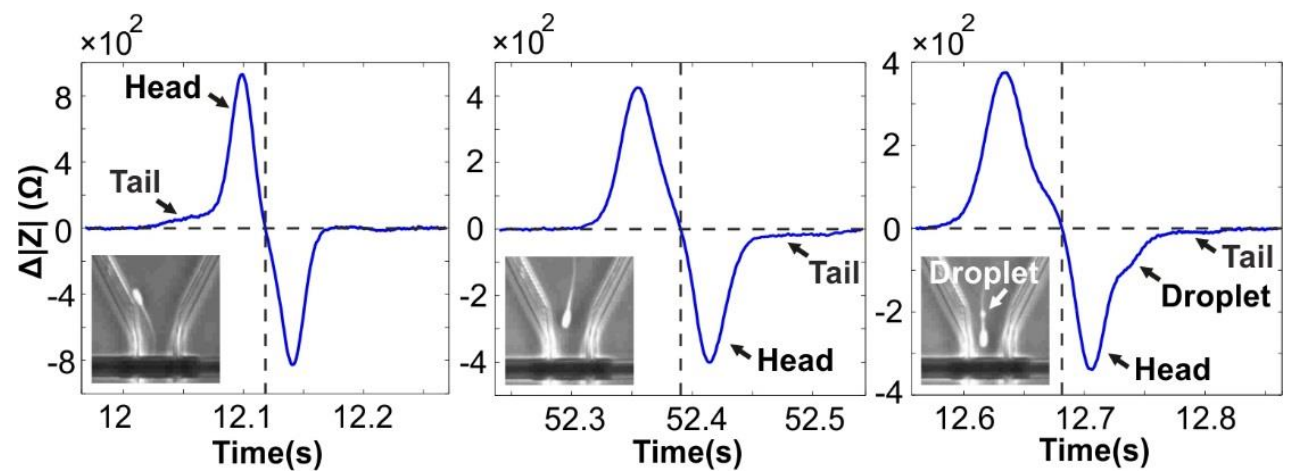

Fig. 6-7: Differential impedance curve of sperm cells in A) tail-first and B) head-first orientation, showing the recorded impedance change upon passing of a sperm cell in between the differential electrode pair over time. C) When a sperm cell contained a cytoplasmic droplet, a distinct feature was observed in the impedance curve. 
Although one would expect a similar response in impedance change when the sperm cell is passing the first electrode pair, the electrical overlap between the two electrode pairs interfered due to which the observed effect was less pronounced. Therefore, we focused on the analysis of the second peak to analyse droplet content.

Cytoplasmic droplet content was investigated by analysing the area under the curve (AUC) of the differential impedance data. In total, 18 morphologically normal and 18 droplet-containing sperm cells were selected for analysis, which crossed the electrodes in head-first orientation. Using Matlab, the maximum (Fig. 6-8A, point A), minimum (point B) and zero crossing point (point C) were determined. Subsequently, the AUC of the positive and negative peak were calculated by performing a numerical integration in Matlab. When comparing the AUC means of the negative peaks of the two populations using a (paired sample) t-test (Table 6$3)$, no statistical difference was found ( $p=0.53)$.

Table 6-3: AUC analysis of sperm cells (head-first) without (control) and with cytoplasmic droplets (both populations, $\mathrm{n}=18$ )

\begin{tabular}{c|cc|cc} 
& $10^{4} \mathrm{AUC}$ & $10^{4} \mathrm{SD}$ & $10^{3} \mathrm{AUC} \mathrm{C}_{\mathrm{n}}$ & $10^{2} \mathrm{SD}_{\mathrm{n}}$ \\
\hline Control & 2.96 & 1.50 & 5.05 & 7.25 \\
Droplet & 2.6 & 1.58 & 6.98 & 23.6 \\
\hline & & $\mathrm{p}=0.53$ & & $\mathbf{p}=\mathbf{0 . 0 0 3}^{*}$
\end{tabular}

A plausible explanation is the effect of the cell orientation, location and velocity on the AUC. The orientation (e.g. cell tilting) and location influence the peak height. Furthermore, the cell velocity has an effect on the peak width. Therefore, the AUC was normalized ( $A \cup C n$, Fig. 6-8A) using the peak height $\left(Y_{B}\right)$ and peak width $\left(X_{B}\right)$. $X_{B}$, the time interval between the zero crossing point $C$ and the peak minimum $B$, was chosen to correct for the cell velocity. Due to the channel constriction close to the first electrode pair, the sperm cells were accelerating when they passed the first electrode pair. At the second electrode pair, their velocity was more constant. Therefore, time interval $X_{B}$ was chosen for peak normalization. After correction for the peak height and peak width ( $A \cup C$ divided by $Y_{B}$ and $X_{B}$ ), a significant difference was found between the AUC's of both populations $(p=0.003)$. 
A threshold value between the populations of sperm cells with and without cytoplasmic droplets (droplet and control, respectively) was chosen in order to count the amount of cells, which were positively and negatively identified based on the AUCn (Fig. 6-8B table insert). This threshold value was chosen to find the highest amount of cells with cytoplasmic droplet content. An AUCn value higher or lower than the threshold value is denoted as AUCn+ or AUCn-, respectively. In total, 17 out of 18 sperm cells with cytoplasmic droplet were positively identified (true positive, or TP), yielding a sensitivity of 0.94 . For the control cells, 8 of 18 were negatively identified as cells with droplet content (false positive, or FP), yielding a specificity of 0.56 . Furthermore, the positive and negative predictive values were 0.68 and 0.91 , respectively. The receiver operating characteristic (ROC) curve was determined by varying the threshold value and calculating the sensitivity and specificity, correspondingly. The area under the ROC curve ( $A \cup C_{R O C}$ ) was 0.85 .

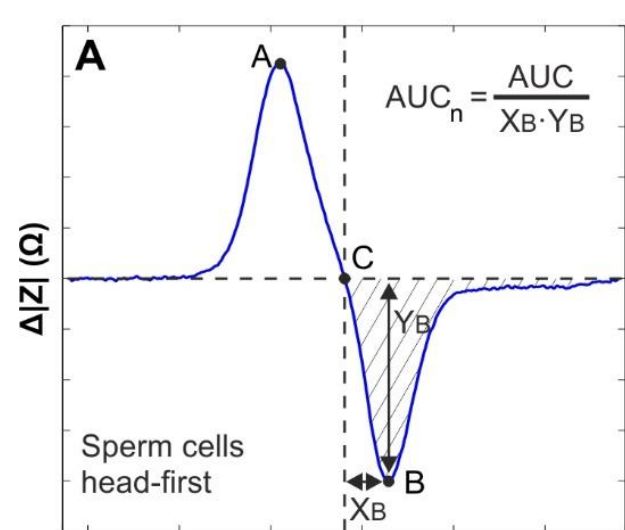

Time (s)

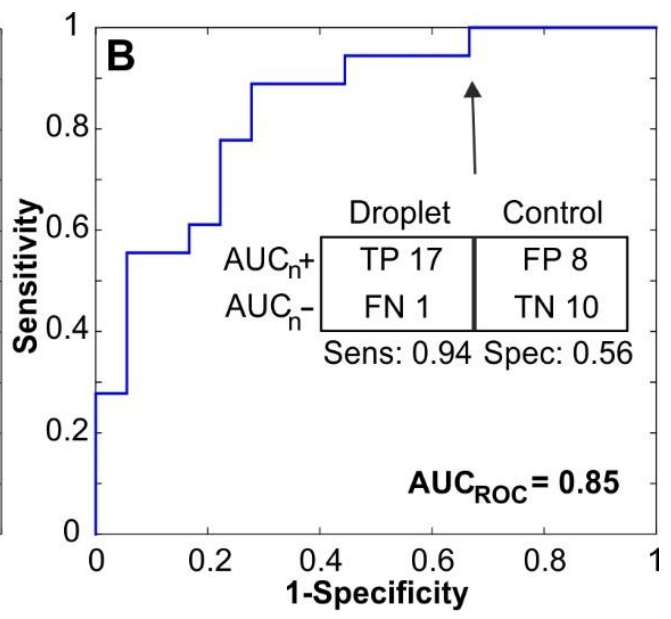

Fig. 6-8: Analysis of cytoplasmic droplet content of sperm cells passing the electrodes head-first based on $A$ ) calculation of the area under the curve (AUC). The first (positive) peak, zero-crossing point and second (negative) peak are denoted as A, C and B, respectively. Peak heights $\left(Y_{B}\right)$ and width $\left(X_{B}\right)$ were used for normalization of the $A U C$, yielding $A U C n$. B) Based on a threshold value of AUCn, the amount of positively identified droplet-containing sperm cells (true positive, or TP) and positively identified cells without a droplet (true negative, or TN) were used to calculate the sensitivity and specificity of electric cytoplasmic droplet detection. The receiver operating characteristic curve (ROC) showed an AUC value of 0.85 . 
Besides analysing the AUC, several other methods could be used for droplet detection. Curve fitting using wavelet transform could be a better candidate for analysis of cytoplasmic droplet content on the single cell level [30]. Furthermore, reducing the measurement cell volume by altering the electrode and channel geometry might improve detection. Moreover, better control over the cell position within the microchannel could reduce the effects of cell orientation, location and velocity on the recorded impedance and could improve droplet detection. For example, dielectrophoretic focusing is a potential approach [31].

\subsubsection{Impedance analysis \& sorting of sperm cells}

Active sorting of sperm cells is an essential feature of a microfluidic sperm sorter. Although impedance measurement is a widely used technique for label-free and non-invasive analysis of single cells in microfluidic systems, few reports show its integration with active sorting capability. To our knowledge, no reports have shown application of this approach to a biological sample. Here, we show the development of a label-free cell sorting system based on impedance measurement. For these experiments, sperm cells were analysed using the chip shown in Fig. 6-13A using the measurement setup illustrated in Fig. 6-3B.

\subsubsection{DEP focusing of sperm cells}

Control over cell location and velocity is necessary to perform accurate measurements of the sperm morphology and to control cell sorting after analysis. As shown before, DEP focusing is used to control these parameters. A frequency of $10 \mathrm{MHz}$ was applied to the focusing electrodes (Fig. 6-13A). At this frequency and in a high conductive environment $\left(1.4 \mathrm{~S} \cdot \mathrm{m}^{-1}\right)$, a negative DEP force is exerted on sperm cells [12]. As a result of this DEP force, the sperm cells will move away from high field gradients at the electrode edges to the middle of the microfluidic channel. Furthermore, high frequency excitation is more suitable for non-invasive DEP manipulation of cells compared to DC or low frequency excitation [32].

To show the effect of DEP focusing on the cell location and velocity, sperm cells were flown through the microfluidic channel with a flow rate of $0.025 \mu \mathrm{L} \cdot \mathrm{min}^{-1}$ with and without DEP excitation. Sperm cell trajectories and location were investigated to show the effect of DEP. Without excitation, the sperm cell trajectories were unaffected (Fig. 6-9A). With excitation, the sperm cells were clearly deflected to the middle of the channel (Fig. 6-9B). The cell location and velocity were optically measured right after passing the $20 \mu \mathrm{m}$ electrode pair (Fig. 6-13A). 


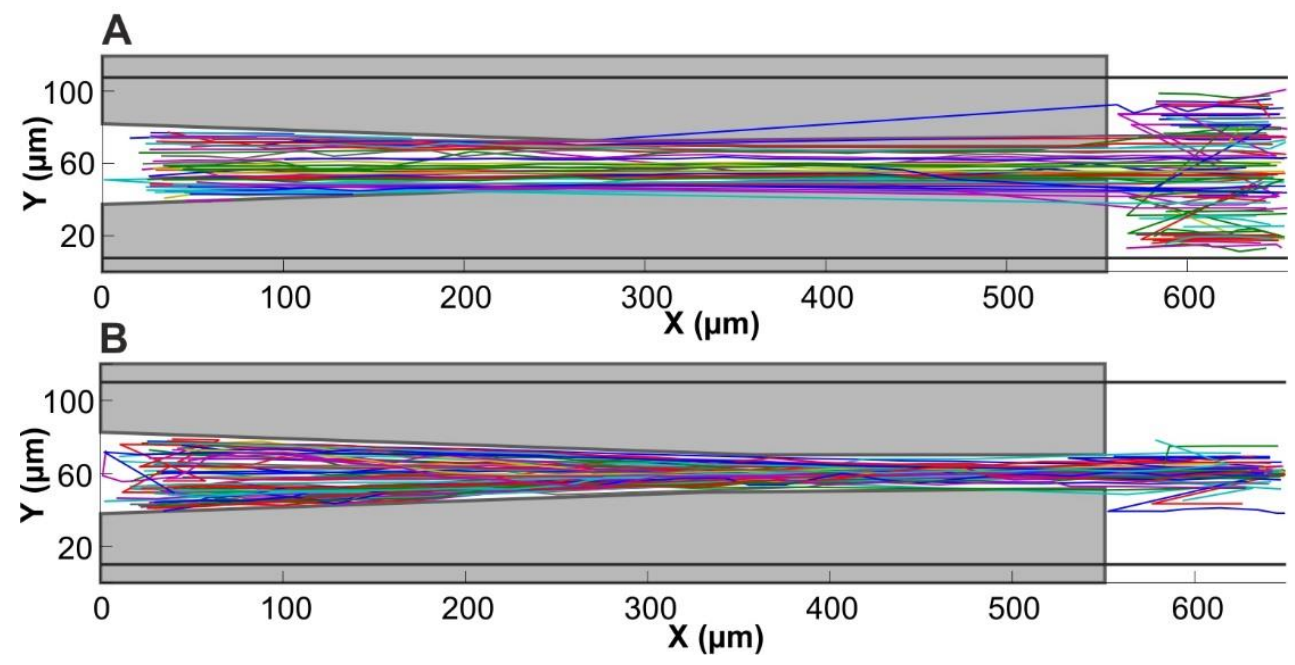

Fig. 6-9: Optical tracking of sperm cells within a $100 \mu \mathrm{m}$ channel A) without and B) with DEP focusing. The channel walls are illustrated by the horizontal black lines, the electrodes are illustrated by the grey coloured areas. Without DEP focusing, the sperm cells exit the focusing area at random Y-location. With focusing, the sperm cells exit the focusing area in the middle of the channel.

Data is presented as a scatterplot of the cell location versus cell velocity with corresponding histograms and boxplots (Fig. 6-10). Without focusing, image analysis showed a broad distribution in both cell location and velocity. With focusing, the width of these distributions was reduced extensively. However, multiple outliers were observed in the boxplots. These data points were caused by sperm cells which got stuck to the channel walls or sperm cells, which pushed themselves away from the middle of the channel by beating their flagella.

\subsubsection{Impedance-controlled sorting of bead and sperm cells}

After detection of a cell with morphological abnormality (e.g. cytoplasmic droplet), it should be removed from the sample. Previous results showed the ability to detect changes in impedance when sperm cells crossed the microelectrodes, to distinguish sperm cells with and without cytoplasmic droplets and to focus the sperm cells using dielectrophoresis to control their location and velocity. A remaining but crucial feature of the microfluidic cell sorter is a sorting algorithm, which accurately controls DEP excitation based on impedance data. 

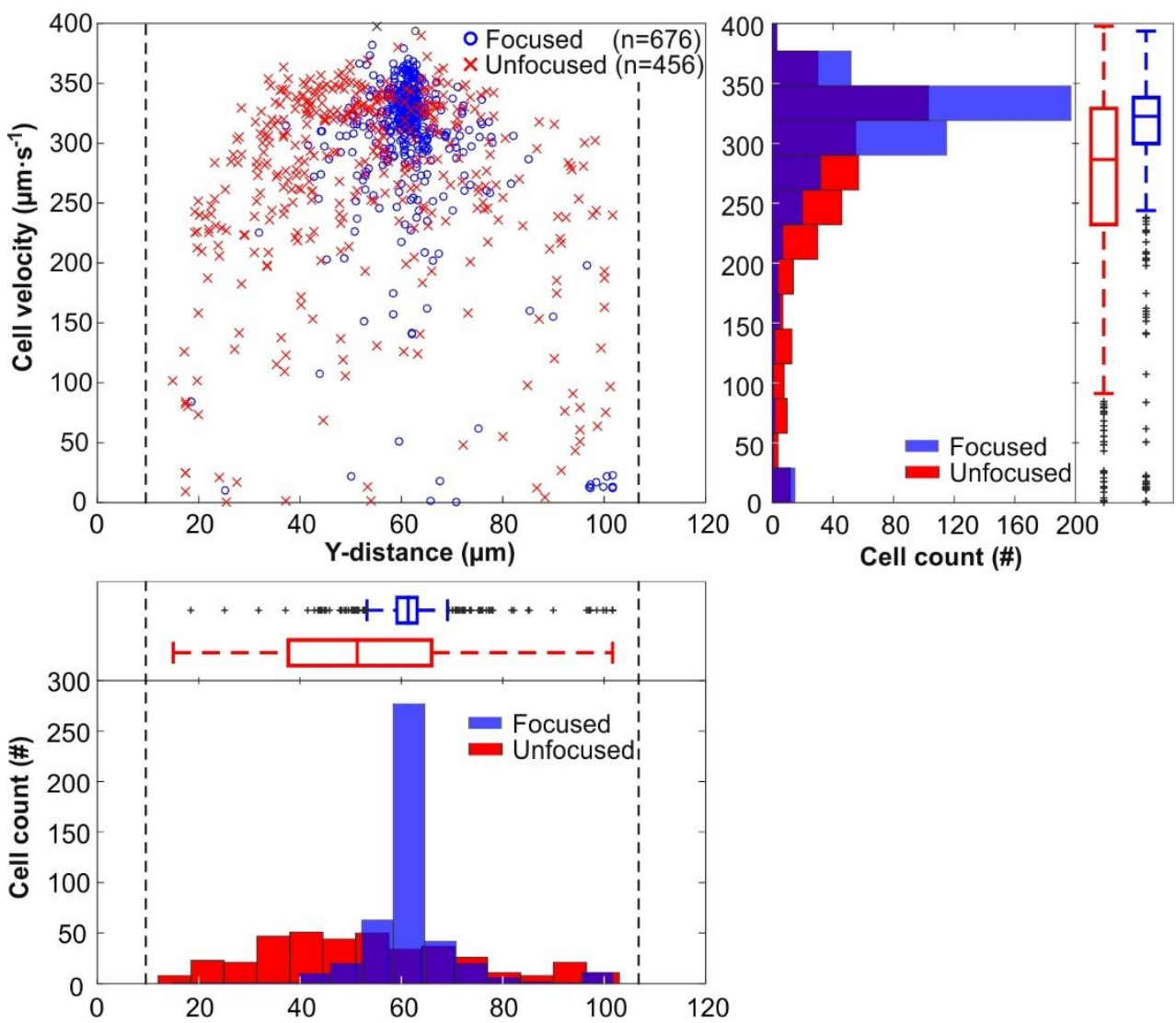

Fig. 6-10: The effect of DEP focusing on sperm location and velocity. Sperm location and velocity were more uniform after focusing the sperm cells in the middle of the channel. The channels walls are situated at $\mathrm{y}=9$ and $\mathrm{y}=107 \mu \mathrm{m}$ (dashed lines).

In a proof of concept experiment, we aim to sort beads and sperm cells based on impedance. For this study, LabVIEW was used to design the sorting algorithm. The process flow of this algorithm is shown in Fig. 6-4. Whenever a change in impedance is recorded, from which the peak shape matches the peak template, the width and the height of the peak are determined. The peak width is used to calculate the particle's velocity in order to predict the ETA at the sorting electrodes. The optically calculated cell velocity, which is used as a reference, is plotted versus the electrically calculated cell velocity (Fig. 6-11). 


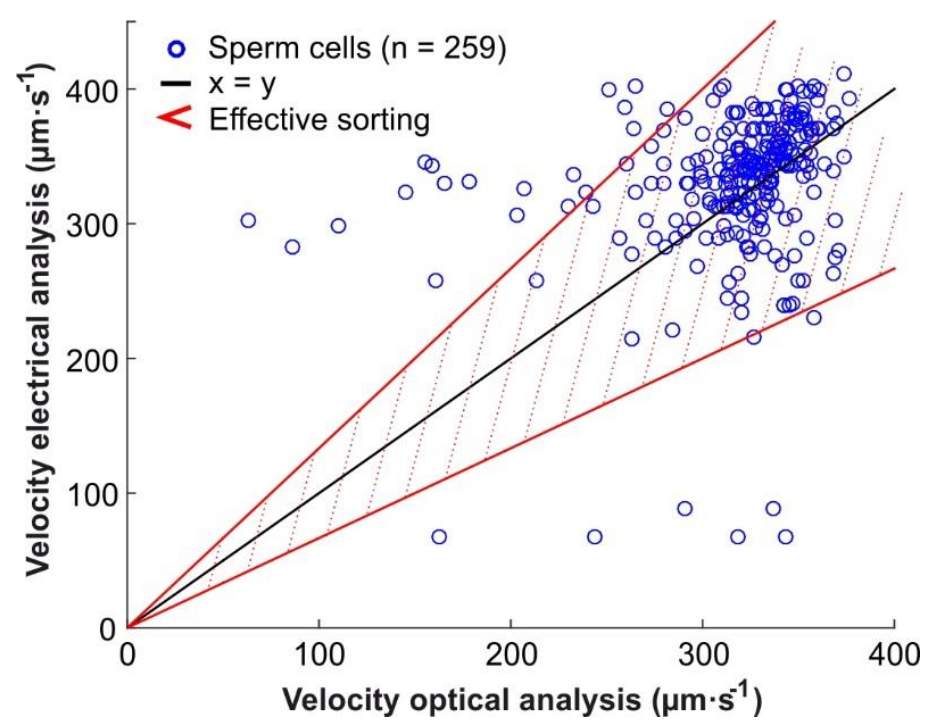

Fig. 6-11: Cell velocity determined by optical ( $x$-axis) and electrical (y-axis) analysis. The majority of points were above the linear line through the origin $(y=x)$, indicating that the electrical measurements overestimate the cell velocity. An inaccuracy of $30 \%$ is taken into account when calculating the ETA and the window of DEP $\left(t_{0} . . . t_{1}\right)$ based on the electrically determined velocity (indicated by red lines). Using this correction, the majority of the points (>90\%) are situated in between the red hatching and thus fall within the window of DEP. Points outside the red hatching correspond to cells which temporarily got stuck to the channel wall.

To correct for the inaccuracy in measured cell velocity (electrically) when determining the window of DEP excitation, a $30 \%$ error margin is built in. This margin indicates that the cell velocity can be underestimated (bottom red line) or overestimated (top red line) by $30 \%$ compared to the optically measured velocity. As a result, all sperm cells, which are positioned in the red hatched region, arrive at the sorting electrodes within the DEP window, resulting in effective sorting. In this example, $3 \mu \mathrm{m}$ polystyrene beads were sorted from sperm cells (Fig. 6-13). A mixture of sperm cells and beads $\left(2 \cdot 10^{6}\right.$ cells and beads $\left.\cdot \mathrm{mL}^{-1}\right)$ was flown through the microfluidic channel at a flow rate of approximately $0.025 \mu \mathrm{L} \cdot \mathrm{min}^{-1}$. The impedance change of a population of sperm cells showed a clear difference compared to a population of beads when crossing the $20 \mu \mathrm{m}$ wide electrodes (Fig. 6-12), allowing discrimination between both species. 


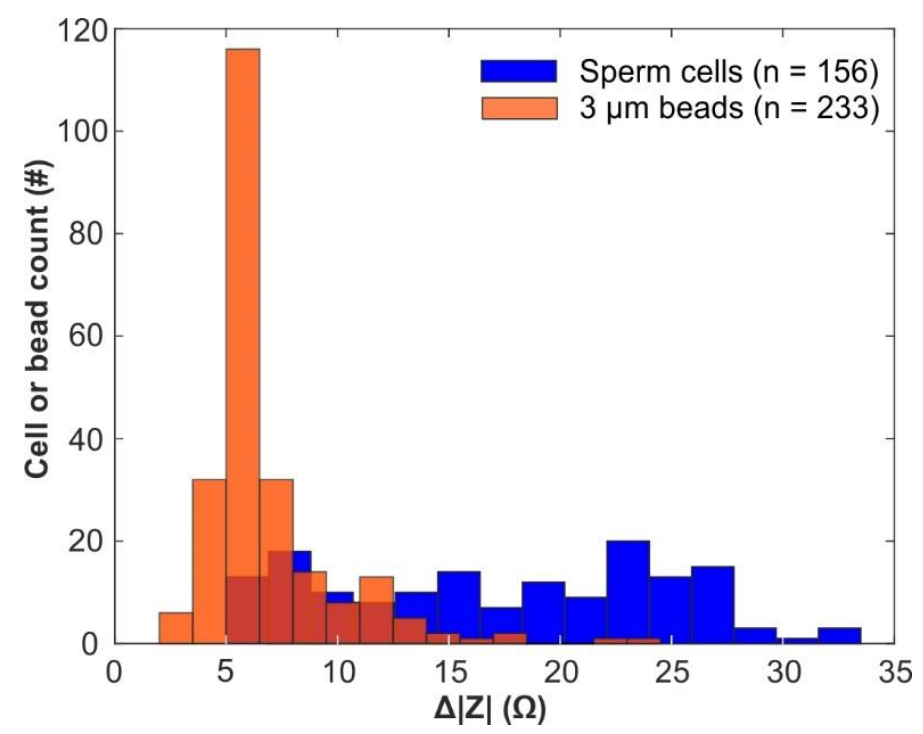

Fig. 6-12: Impedance change distribution of $3 \mu \mathrm{m}$ polystyrene beads and sperm cells measured in a $100 \mu \mathrm{m}$ wide and $20 \mu \mathrm{m}$ high channel with $20 \mu \mathrm{m}$ wide electrodes. A broad distribution in impedance was observed for sperm cells, caused by mutual differences in cell location and orientation.

Whenever an impedance change of a particle was detected, which fitted within the WOI, the DEP electrodes were activated to sort the particle in the top channel. The impedance WOI was set to 4-8 $\Omega$, which matched the impedance change when a bead passed the electrodes. When beads passed the electrodes, the recorded changes in impedance (5.4 $\pm 0.7 \Omega, n=15)$ fitted within the WOI (Fig. 6-13B), consequently sorting the beads actively in the top channel at the channel split (green trajectories, Fig. 6-13C). Whenever sperm cells $(19.6 \pm 5.7 \Omega, n=12)$ or debris $(<4 \Omega$ ) passed the detection electrodes (red triangles and blue squares, respectively), the recorded impedance was above or below the WOI, respectively. As a result, sperm cells and debris were drawn in the bottom channel without being deflected by the sorting electrodes (red and blue trajectories, respectively). For optical verification, the sorting speed in the described experiment was set to $<1$ sperm cell. $\mathrm{s}^{-1}$ using a low bead and sperm concentration and small flow rate. In the reported experiment, the sorting speed is limited to $<5$ cells $\cdot \mathrm{s}^{-1}$ when using the peak fitting algorithm at a sampling frequency of $899 \mathrm{~Hz}$. The sampling frequency can be increased to increase the sorting throughput, although we expect to reach a maximum of 50 cells $\cdot \mathrm{s}^{-1}$ due to limitations in computational speed of the system. 


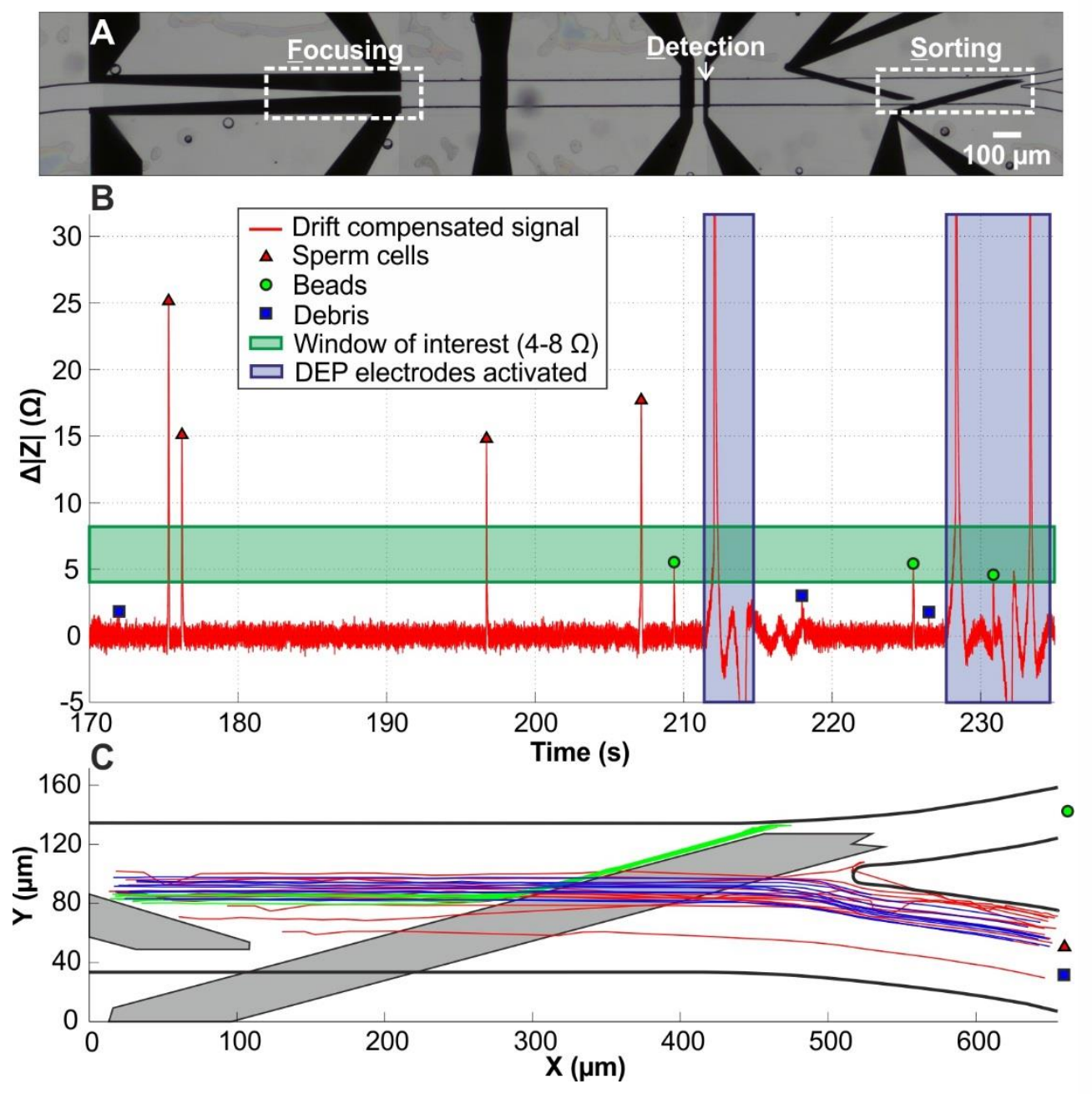

Fig. 6-13: Discrimination and active sorting of beads and sperm cells in A) a chip $100 \mu \mathrm{m}$ channel with integrated electrodes for cell focusing, detection and sorting. B) When a bead passed the detection electrodes and the corresponding impedance change fitted within the window of interest (green window, 4-8 $\Omega$ ), the DEP electrode pair was activated (blue window) to sort the particle. C) Analysis of the bead trajectories showed effective deflection of the beads to the top channel by active sorting, whereas sperm cells and debris were drawn into the bottom channel. 


\subsection{Conclusions}

In this paper, we presented a novel application of impedance measurement to study sperm cells in a microfluidic system. Differential impedance analysis was employed to successfully study the cell orientation and cytoplasmic droplet content, which is a frequently occurring morphological defect in boar sperm cells. Furthermore, we developed a label-free cell sorting system in which cells can be sorted using DEP based on impedance data. In a proof of concept experiment, we were able to sort plastic beads from sperm cells based on corresponding impedance peaks. Combination of both techniques is a promising approach towards sperm refinement applications in the livestock industries. Besides analysis of morphology (droplet content), impedance analysis might be used to investigate other sperm cell parameters, such as cell viability and the internal environment of the cell $[15,16,18]$.

\subsection{Acknowledgements}

Financial support from the NWO - Netherlands Organization of Scientific Research (Spinoza Grant A. van den Berg, Veni L.I. Segerink), the scientific support of A.J. Sprenkels and S. Sukas and the technical support of F. van Rossem, P.M. ter Braak, J.W. van Nieuwkasteele, H.L. de Boer and J.G. Bomer are gratefully acknowledged. We also thank the "KI Twenthe" for the kind supply of boar semen samples.

\subsection{References}

1. Broekhuijse, M.L.W.J., Prediction of porcine male fertility, in Dissertation Faculty of Veterinary Medicine. 2012, Utrecht University.

2. Gadea, J., Sperm factors related to in vitro and in vivo porcine fertility. Theriogenology, 2005. 63(2): p. 431-44.

3. Popwell, J.M. and W.L. Flowers, Variability in relationships between semen quality and estimates of in vivo and in vitro fertility in boars. Anim Reprod Sci, 2004. 81(1-2): p. 97-113.

4. Tsakmakidis, I.A., A.G. Lymberopoulos, and T.A.A. Khalifa, Relationship between sperm quality traits and field-fertility of porcine semen. J Vet Sci, 2010. 11(2): p. 151-4.

5. Kuster, C.E. and G.C. Althouse, The fecundity of porcine semen stored for 2 to 6 days in androhep ${ }^{\circledR}$ and $X$-cell ${ }^{T M}$ extenders. Theriogenology, 1999. 52(3): p. 365-76.

6. Shipley, C.F., Breeding soundness examination of the boar. Swine Health Prod, 1999. 7(3): p. 117-20. 
7. Kaplan, M., et al., Boar sperm cytoplasmic droplets: Their ultrastructure, their numbers in the epididymis and at ejaculation and their removal during isolation of sperm plasma membranes. Tissue Cell, 1984. 16(3): p. 455-68.

8. Cooper, T.G., Cytoplasmic droplets: the good, the bad or just confusing? Hum Reprod, 2005. 20(1): p. 9-11.

9. Waberski, D., et al., Fertility of long-term-stored boar semen: Influence of extender (Androhep and Kiev), storage time and plasma droplets in the semen. Anim Reprod Sci, 1994. 36(1): p. 145-51.

10. Cho, B.S., et al., Passively Driven Integrated Microfluidic System for Separation of Motile Sperm. Anal Chem, 2003. 75(7): p. 1671-5.

11. Matsuura, K., et al., Screening of sperm velocity by fluid mechanical characteristics of a cyclo-olefin polymer microfluidic sperm-sorting device. Reprod Biomed Online, 2012. 24(1): p. 109-15.

12. Ohta, A.T., et al., Motile and non-motile sperm diagnostic manipulation using optoelectronic tweezers. Lab Chip, 2010. 10(23): p. 3213-7.

13. Rosales-Cruzaley, E., et al., Sperm cells manipulation employing dielectrophoresis. Bioproc Biosyst Eng, 2013. 36(10): p. 1353-62.

14. Seo, D.-b., et al., Development of sorting, aligning, and orienting motile sperm using microfluidic device operated by hydrostatic pressure. Microfluid Nanofluid, 2007. 3(5): p. 561-70.

15. David, F., et al., Viability and membrane potential analysis of Bacillus megaterium cells by impedance flow cytometry. Biotechnol Bioeng, 2012. 109(2): p. 483-92.

16. Haandbaek, N., et al., Characterization of subcellular morphology of single yeast cells using high frequency microfluidic impedance cytometer. Lab Chip, 2014. 14(2): p. 369-77.

17. Holmes, D., et al., Leukocyte analysis and differentiation using high speed microfluidic single cell impedance cytometry. Lab on a Chip, 2009. 9(20): p. 2881-2889.

18. Kirkegaard, J., et al., Study of Paclitaxel-Treated HeLa Cells by Differential Electrical Impedance Flow Cytometry. Biosensors, 2014. 4(3): p. 257-72.

19. Segerink, L.I., et al., On-chip determination of spermatozoa concentration using electrical impedance measurements. Lab on a Chip, 2010. 10(8): p. 1018-1024.

20. Han, S.-I., Y.-D. Joo, and K.-H. Han, Impedance-activated microseparator based on amplitude modulation sensing and dielectrophoretic switching methods. Sensor Actuat B-Chem, 2012. 171-172: p. 1312-20.

21. Hawkings, B.G., J.P. Gleghorn, and B.J. Kirby, "Dielectrophoresis for cell and particle manipulations" in Methods in Bioengineering: Biomicrofabrication and Biomicrofluidics. 2009: Artech Press. 
22. Cetin, B. and D.Q. Li, Dielectrophoresis in microfluidics technology. Electrophoresis, 2011. 32(18): p. 2410-27.

23. Pethig, R., Review Article-Dielectrophoresis: Status of the theory, technology, and applications. Biomicrofluidics, 2010. 4(2): p. 022811.

24. Fuhr, G., et al., High-frequency electric field trapping of individual human spermatozoa. Hum Reprod, 1998. 13(1): p. 136-41.

25. Clements, J.D. and J.M. Bekkers, Detection of spontaneous synaptic events with an optimally scaled template. Biophy J, 1997. 73(1): p. 220-9.

26. Sun, T. and H. Morgan, Single-cell microfluidic impedance cytometry: $a$ review. Microfluid Nanofluid, 2010. 8(4): p. 423-43.

27. Petrunkina, A.M., et al., Signalling pathways involved in the control of sperm cell volume. Reproduction, 2007. 133(1): p. 61-73.

28. Saravia, F., et al., Differences in boar sperm head shape and dimensions recorded by computer-assisted sperm morphometry are not related to chromatin integrity. Theriogenology, 2007. 68(2): p. 196-203.

29. Thurston, L.M., P.F. Watson, and W.V. Holt, Sources of variation in the morphological characteristics of sperm subpopulations assessed objectively by a novel automated sperm morphology analysis system. J Reprod Fertil, 1999. 117(2): p. 271-80.

30. Li, Y.-I., et al., Continuous Wavelet Transform to Improve Resolution of Overlapped Peaks Based on Curve Fitting. Spectrosc Lett, 2013. 46(7): p. 507-15.

31. Holmes, D., H. Morgan, and N.G. Green, High throughput particle analysis: Combining dielectrophoretic particle focussing with confocal optical detection. Biosens Bioelectron, 2006. 21(8): p. 1621-30.

32. Qian, C., et al., Dielectrophoresis for Bioparticle Manipulation. Int J Mol Sci, 2014. 15(10): p. 18281-309. 


\section{Sperm quality after DEP manipulation}

Chapter 6 reported on the use of DEP for focusing and sorting of sperm cells in a microfluidic platform. Although the effects of DEP on cell viability have been described for a variety of cell types, the effects on sperm cells have not been studied in detail. Therefore, to investigate whether DEP has a negative influence on sperm quality, sperm cells were exposed to electrical fields, typically used in DEP experiments. After exposure, the sperm cells were collected and fluorescently stained to investigate the effects of the electrical fields on the mitochondrial membrane potential and the integrity of the plasma and acrosomal membranes.

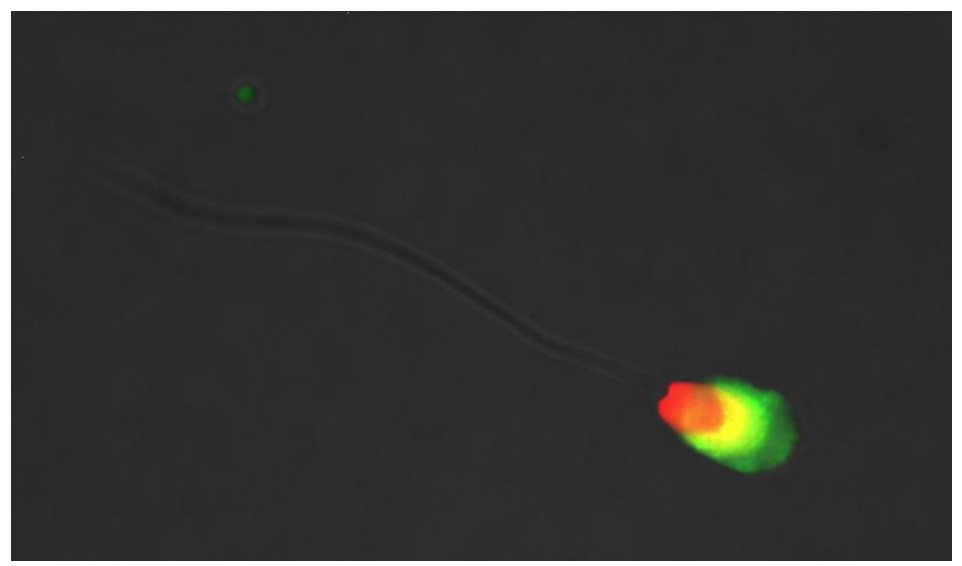

Sperm cell with damaged cell membrane and acrosome. (photo N. Huisintveld)

Manuscript in preparation: de Wagenaar, B., van Etten, J., Olthuis, W., van den Berg, A., Segerink, L.I., Sperm quality after DEP manipulation. 


\subsection{Introduction}

In the last decennium, a growing number of reports showed the potential to perform sperm manipulation and selection in microfluidic systems [1]. Microfluidic systems have shown important advantages over traditional selection techniques [2] due to small sample volumes, short processing times and the potential to manipulate cells on the single cell level. A good example is reported by Cho et al. [3]. They showed the ability to sort motile from non-motile sperm cells and cell debris in a microfluidic platform by making use of the swimming capacity of motile sperm cells to swim through the streamlines of a laminar flow system. A similar approach was used by Seo et al. [4] in which only motile sperm cells were able to swim against the flow created by a hydrostatic pressure difference in a microchannel. This principle was also applied by Lopez-Garcia et al. [5] and Qui et al. [6] using a single channel with a pressure-driven flow to isolate motile sperm cells by their tendency to swim close to surfaces and against the flow. A combination of this phenomenon and electric detection of the motile sperm concentration is reported by Chen et al. [7]. A good correlation was found between the sample's sperm motility index and the measured motile sperm concentration on-chip.

A different way to manipulate cells in microfluidic channels is DEP, in which the dielectric properties of the cells are used to isolate, enrich and sort them [8, 9]. DEP describes the force which acts on a cell which is exposed to a non-uniform electric field (paragraph 3.4). This force can either be attractive (pDEP) or repulsive (nDEP). Some reports have shown the employment of DEP to isolate and entrap sperm cells in a microfluidic setting. Fuhr et al. [10] used nDEP to trap sperm cells in an octopole electrode system and an interdigitated electrode configuration. Cell trapping was performed using high frequency excitation at $10 \mathrm{MHz}$ to minimize induced membrane potentials. Field strengths up to $100 \mathrm{kV} \cdot \mathrm{cm}^{-1}$ were reported to be well tolerated at this frequency. Despite of this, they observed temporary cellular paralysis when trapping sperm cells in nDEP octopole field cages $(8 \mathrm{MHz}, 2.5 \mathrm{~V})$. They explained this observation as the dysfunction of the mitochondrial system by field-induced charges at the interfaces of the mitochondrial membranes, disrupting a functional mitochondrial membrane potential [10]. Enrichment and isolation of mature and spermatogenic cells by iDEP was performed by Rosales-Cruzaley et al. [11]. Insulating structures within the channel were used to apply nDEP forces on the cells. By applying different potentials ranging between 200-1500 V, selective trapping of mature and spermatogenic cells was obtained based on differences in 
cell shape. The sperm viability was shown to decrease significantly with increasing DC potentials between 0 and $1000 \mathrm{~V}$ when sperm cells were retained near the iDEP structures for 1 minute [11]. An explanation for this rapid decreasing cell viability is the use of DC fields, which are known to induce high membrane potentials and inflict membrane damage and cell lysis, consequently. Another application of DEP for sperm manipulation is presented in chapter 6 of this thesis. This chapter described the use of nDEP for focusing of sperm cells and impedance-based sorting of beads from a mixture of sperm cells and beads. This approach might be employed for sperm selection based on cell morphology.

Although DEP has been used for non-invasive manipulation of cells, it can inflict cell damage by inducing high transmembrane potentials (TMP) and by creating high fluid temperatures above physiological temperature $\left(>37^{\circ} \mathrm{C}\right)$ [9]. Voldman [9] suggested that DEP exposure in the $>\mathrm{MHz}$ range and $10^{\prime} \mathrm{s} \mathrm{kV} \cdot \mathrm{m}^{-1}$ does not affect cell viability and function to a big extent, although long-term electric field exposure and direct contact with the electrodes should be avoided. However, no extensive literature is available describing the effects of DEP on sperm quality.

To investigate whether DEP can safely be employed for sperm selection and manipulation, the effects of electric fields on sperm cells were investigated in a microfluidic setting. A microfluidic chip, capable of focusing, detecting and sorting sperm cells using parallel microelectrodes as described in paragraph 6.2.1, was used for this study. After exposure to electrical fields, typically used in nDEP experiments, the plasma, acrosomal and mitochondrial membranes were investigated. The integrity of these membranes has a major influence on the function and the fertilization potential of sperm, as described in a review of Silva and Gadella [12].

\subsection{Materials \& methods}

\subsubsection{Chip fabrication}

Microfluidic chips (Fig. 6.1A) were fabricated using routine photolithography wet etch, sputter and bonding techniques as described in paragraph 6.2.1. In short, microelectrodes were fabricated on two borofloat glass wafers (BF33, $100 \mathrm{~mm}$ diameter, 500 and $1100 \mu \mathrm{m}$ thick) after photoresist deposition, exposure and development, BHF wet-etching, sputtering titanium/platinum (30 and $120 \mathrm{~nm}$, respectively) and resist lift-off. After powderblasting, a layer of PerMX3020 (Dupont, $20 \mu \mathrm{m}$ ) was laminated and developed on the $500 \mu \mathrm{m}$ thick BF33 wafer.

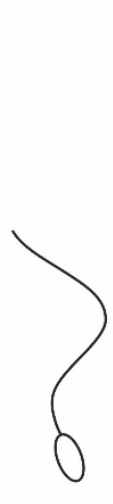


Subsequently, the two wafers were aligned in an anodic bonder (EV-501) and bonded together using a heated press (Carver). After dicing (Dicing saw Disco DAD 321), the chips were ready for experimentation.

The chip, used for the described experiments, consisted of a $20 \mu \mathrm{m}$ high and 100 $\mu \mathrm{m}$ wide channel, in which a parallel-oriented electrode pair, with an electrode width of $50 \mu \mathrm{m}$, was used for DEP excitation (Fig. 7-1). Each reported experiment was performed with the same chip, which was cleaned and prepared before each experiment as described in paragraph 7.2.2.

\subsubsection{Chip \& sample preparation}

Fresh boar semen was obtained from a local artificial insemination centre ("KI Twenthe", The Netherlands) at a concentration of $20 \cdot 10^{6}$ cells $\cdot \mathrm{mL}^{-1}$. The samples were diluted with Beltsville Thawing Solution (BTS, Solusem, Aim Worldwide) to a concentration of $10 \cdot 10^{6} \mathrm{cells} \cdot \mathrm{mL}^{-1}$.

Before each experiment, the microfluidic channel was cleaned using a $1 \%$ detergent (Alconox, Sigma Aldrich) in demiwater, by flushing the solution through the channels for $30 \mathrm{~min}$ at a flow rate of $1 \mu \mathrm{L} \cdot \mathrm{min}^{-1}$ using a syringe pump (neMESYS, Cetoni $\mathrm{GmbH}$ ) equipped with a Hamilton gastight syringe (1710N). After removing the detergent solution with demiwater, the channels were flushed with a 100 $\mu \mathrm{g} \cdot \mathrm{mL}^{-1}$ poly(L-lysine)-grafted-poly(ethylene glycol) (PLL(20-g[3.5]-PEG(2), SuSoS) in demiwater solution for $30 \mathrm{~min}$ at $1 \mu \mathrm{L} \cdot \mathrm{min}^{-1}$ to create a hydrophilic coating on the channel walls to prevent cell adhesion [13]. Subsequently, BTS was flushed through the channel to remove excess coating solution for $30 \mathrm{~min}$ at $1 \mu \mathrm{L} \cdot \mathrm{min}^{-1}$. Subsequently, sperm solution was flushed through the channel at a flow rate of 0.2 $\mu \mathrm{L} \cdot \mathrm{min}^{-1}$ during experiments.

\subsubsection{On-chip DEP exposure \& sample collection}

To investigate potential cell damage by DEP manipulation, sperm cells were exposed to electrical fields, typically used for a DEP experiments (paragraph 6.2.5). The setup is shown in Fig. 7-1.

A chipholder (Fig. 6.1B) was used to connect the microelectrodes to an Agilent function generator, which applied a potential to the microelectrodes. Fused silica capillaries (ID $150 \mu \mathrm{m}$, OD $360 \mu \mathrm{m}$, Polymicro Technologies), which were connected to Nanopore connectors that were screwed into the chipholder, were used to connect the syringe and the sample collection tube to the microfluidic channel. After sample injection and transport through the microfluidic channel, the 


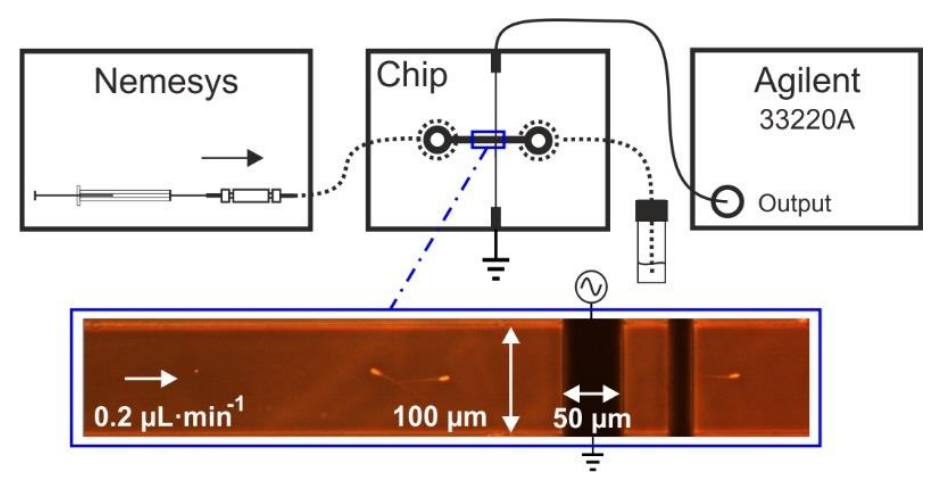

Fig. 7-1: Setup for DEP manipulation. Sperm cells were flown trough a $20 \mu \mathrm{m}$ high and $100 \mu \mathrm{m}$ high microfluidic channel at a flow rate of $0.2 \mu \mathrm{L} \cdot \mathrm{min}^{-1}$ using a neMESYS syringe pump. An Agilent function generator was used for DEP excitation of the $50 \mu \mathrm{m}$ wide parallel-oriented microelectrodes. Sperm cells were collected in a $25 \mathrm{~cm}$ long glass capillary finally connected to a collection tube.

outflowing sample was first collected in a small tube using this set-up. Initial control experiments showed a big decrease in viability of the collected sperm cells. Evaporation of the solution was found to be the cause of cell damage. Although the collection tube was closed off with a septum, the total collection volume was so small $\left(6 \mu \mathrm{L} \cdot 30 \mathrm{~min}^{-1}\right)$ that evaporation still played a major role. An alternative was to collect the outflowing sperm in a collection tube, prefilled with a large volume of BTS, minimizing the effect of evaporation. However, this would lead to high dilution of the sample, yielding reduced sperm concentrations and decreased analysis throughput. Centrifugation could be an option to increase this concentration, but it is known to inflict cell damage [20,21]. In our final approach, sperm was collected which was present in the capillary between the chip and the collection tube, which was not affected by evaporation. This capillary was $25 \mathrm{~cm}$ long and contained approximately $4.5 \mu \mathrm{L}$ sperm solution. This volume was separated into three batches after which three fluorescent staining procedures were performed.

\subsubsection{Fluorescent staining procedures}

The sperm quality was investigated by three fluorescent staining procedures. In total, $1.5 \mu \mathrm{L}$ staining solution was added to $1.5 \mu \mathrm{L}$ sperm solution for each of the three stainings. Fluorescence was recorded using an EVOS FL Cell imaging system equipped with red fluorescent protein (RFP) and green fluorescent protein (GFP) filter blocks. 
The membrane integrity was investigated using a SYBR14/Propidium iodide (PI) live/dead staining. Sperm cells were incubated in 1000x dilution of SYBR14 (stock 1 $\mathrm{mM}$, ex/em 488/518 nm, Life Technologies) and a 100x dilution of PI (stock $2.4 \mathrm{mM}$, ex/em 535/617 nm, Life Technologies) for $5 \mathrm{~min}$ at room temperature. Green or red nuclei staining indicated sperm cells with intact or damaged plasma membranes, respectively.

The integrity of the acrosome was tested using fluorescein conjugated Pisum Sativum Agglutinin (FITC-PSA, ex/em 422/544, Vector Laboratories). Sperm cells were incubated in a 100x dilution of FITC-PSA (stock $5 \mathrm{mg} \cdot \mathrm{mL}^{-1}$ ) in BTS for $30 \mathrm{~min}$ at 37 ㅇ. $\mathrm{PSA}$ is a lectin, which has specificity toward acrosomal matrix glycoproteins, or specifically, toward a-linked mannose-containing oligosaccharides, which are components of the matrix glycoproteins $[12,14]$. These glycoproteins are exposed when the acrosome is damaged or during the acrosomal reaction. As a result, damaged or reacted acrosomes are stained green by FITC.

The mitochondrial membrane potential (MMP) was evaluated after staining with 5,5',6,6'-tetrachloro-1,1',3,3'-tetraethylbenzimidazolylcarbocyanine iodide (JC-1, ex/em 488/525-590 nm, Life Technologies). Sperm cells were incubated in $2 \mu \mathrm{M}$ JC1 in BTS solution for $30 \mathrm{~min}$ at $37^{\circ} \mathrm{C}$. Mitochondria with a high membrane potential are stained orange/red, whereas mitochondria with a low membrane potential are stained green or remained unstained (when membranes are completely depolarized) $[12,15]$.

\subsubsection{TMP model}

Exposing cells to high TMPs can lead to membrane electroporation and rapid cell death [9]. The built-up of this potential was simulated using a numerical model in MATLAB (version R2014b, MathWorks). This model is based on equations formed by Pauly and Schwann [16], which describe the two characteristic relaxation mechanisms for biological cells due to polarization of the cell membraneelectrolyte interface and the polarization between the electrolyte and cell cytoplasm. The relaxation of the cell membrane-electrolyte interface $\tau(s)$ is described by [9]:

$$
\tau=\frac{r C_{m, 0}\left(\frac{1}{\sigma_{i}}+\frac{1}{2 \sigma_{e l}}\right)}{1+r G_{m, 0}\left(\frac{1}{\sigma_{i}}+\frac{1}{2 \sigma_{e l}}\right)}
$$

in which $r$ is the radius of the cell $(\mathrm{m}), \sigma_{i}$ the conductivity of cell cytoplasm $\left(\mathrm{S} \cdot \mathrm{m}^{-1}\right)$ 
and $\sigma_{e l}$ the conductivity of the electrolyte $\left(\mathrm{S} \cdot \mathrm{m}^{-1}\right) . C_{m, 0}$ and $G_{m, 0}$ are the specific membrane capacitance $\left(\mathrm{F} \cdot \mathrm{m}^{-2}\right)$ and conductance $\left(\mathrm{S} \cdot \mathrm{m}^{-2}\right)[9]$ :

$$
C_{m, 0}=\frac{\varepsilon_{m} \varepsilon_{0}}{d_{m}} \quad \text { and } \quad G_{m, 0}=\frac{\sigma_{m}}{d_{m}}
$$

in which $\varepsilon_{m}$ is the membrane permittivity, $\varepsilon_{0}$ the vacuum permittivity $\left(8.86 \cdot 10^{-12}\right.$ $\left.\mathrm{F} \cdot \mathrm{m}^{-1}\right), \sigma_{m}$ the membrane conductivity $\left(\mathrm{S} \cdot \mathrm{m}^{-1}\right)$ and $d_{m}$ the cell membrane thickness (m). Subsequently, the induced the transmembrane potential $\left|V_{T M}\right|(\mathrm{V})$ is calculated by [9]:

$$
\left|V_{T M}\right|=\frac{1.5|E| r}{\sqrt{1+(\omega \tau)^{2}}}
$$

where $|E|$ is the maximal electrical field $\left(\mathrm{V} \cdot \mathrm{m}^{-1}\right) \cdot \ln$ this model, a cell was considered as a single shelled spheroid. However, the shape of a sperm cell resembles a triaxial ellipsoid rather than a round sphere. Therefore, this model provided a rough estimation of the induced TMP. For this simulation, the sperm surface area (34.5 $\left.\mu \mathrm{m}^{2}\right)$ [17] was used to calculate the radius $(1.7 \mu \mathrm{m})$ of a sphere with equal crosssectional area. Simulations were performed using a field amplitude of $6 \mathrm{~V}$ and an interelectrode distance of $20 \mu \mathrm{m}\left(300 \mathrm{kV} \cdot \mathrm{m}^{-1}\right)$.

\subsection{Results \& discussion}

\subsubsection{TMP model}

When manipulating cells using DEP, two factors can inflict cell damage: high TMPs and high fluid temperatures by Joule heating. When exposing cells to an electrical field, charge accumulation results in an induced potential over the cell membrane, which is known as the TMP. When this potential reaches a value between 0.2-1.5 V [9], the cell membrane starts to electroporate, resulting in rapid cell death. Besides high TMPs, temperature increase of the solution by Joule heating can inflict cell damage [9].

To investigate whether high TMPs are induced on the sperm cells, a theoretical model is constructed as described in paragraph 7.2.5. First, the induced TMP was modelled using a numerical model. This model, which is based on the relaxation mechanism of the cell membrane-electrolyte interface, simulated the induced TMP as a function of field frequency (Fig. 7-2). At low frequencies $(<1 \mathrm{MHz})$, a high transmembrane potential $( \pm 1.5 \mathrm{~V})$ was observed due to charging of the cell 
membrane. This potential started to drop at frequencies above $100 \mathrm{kHz}$. At $10 \mathrm{MHz}$, which is a typical frequency used for DEP experiments, the TMP dropped to approximately $0.28 \mathrm{~V}$, which is below the $0.2-1.5 \mathrm{~V}$ range [9]. Based on this model, no cell damage is expected when exposing sperm cells to high frequency electrical fields $(10 \mathrm{MHz})$ required for DEP sorting applications. At low frequencies, cell damage is expected to occur due to high TMPs, which has been described in literature as well [9].

The second potential cause of cell damage is Joule heating. No simulation of Joule heating was constructed to investigate the temperature increase inside the channel. When working at room temperature, the fluid temperature is well under the physiological temperature $\left(37^{\circ} \mathrm{C}\right)$ if no excessive fluid heating is taking place $(<$ $10^{\circ} \mathrm{C}$ ) at which no cell damage is expected.

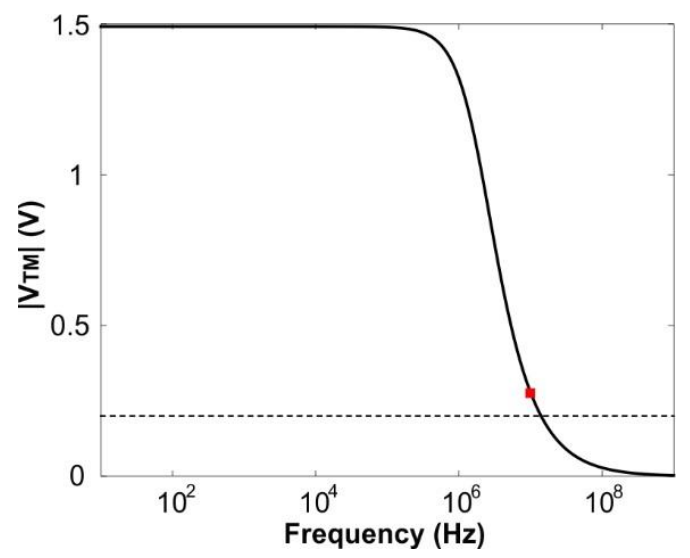

Fig. 7-2: Simulation of the TMP. At low field frequencies, the cell membrane is polarized by the electrical field and the induced transmembrane potential is high $(1.5 \mathrm{~V})$. When the field frequency increased above $1 \mathrm{MHz}$, the induced transmembrane potential decreased. At $10 \mathrm{MHz}$, the TMP is approximately $0.28 \mathrm{~V}$ (red square), which is slightly higher than the $0.2 \mathrm{~V}$ limit (dashed line). The following parameters were used: $s=20 \mu \mathrm{m},|E|=300 \mathrm{kV} \cdot \mathrm{m}^{-1}$, $A_{\text {sperm }}=34.5 \mu \mathrm{m}^{2}, d_{m}=5 \cdot 10^{-9} \mathrm{~m}, \sigma_{e l}=1.4 \mathrm{~S} \cdot \mathrm{m}^{-1}, \sigma_{i}=$ $0.4 \mathrm{~S} \cdot \mathrm{m}^{-1}, \sigma_{m e m}=10^{-8} \mathrm{~S} \cdot \mathrm{m}^{-1} \cdot \varepsilon_{e l}=80$. 


\subsubsection{Sperm quality}

Potential cell damage by DEP was investigated by assessing the integrity of the plasma, acrosomal and mitochondrial membranes. The integrity of the plasma membrane was visualized by SYBR/PI staining (Fig. 7-3A). Sperm cells with intact plasma membranes stained green, whereas cells with a damaged membrane stained red. Complete deterioration of sperm function occurs upon damage to the plasma membrane. The integrity of the acrosomes was investigated using FITC-PSA (Fig. 7-3B), which binds to exposed matrix glycoproteins of damaged or reacted acrosomes. Sperm cells with damaged or reacted acrosomes are not able to undergo the acrosomal reaction, which is an essential process in the fertilization of an egg cell in vivo and in vitro (IVF). The mitochondrial function was determined by JC-1 staining (Fig. 7-3C). At low mitochondrial membrane potential, JC-1 is mainly present in its monomeric form, yielding a green fluorescent signal for depolarized membranes of mitochondria, present in the midpiece of the sperm cells. At high MMP, JC-1 forms aggregates, resulting in orange/red excitation. Cells with unstained midpieces contain mitochondria with completely depolarized membranes, yielding no fluorescent excitation [15]. Mitochondrial dysfunction, characterized by green or absent JC-1 staining, has been related to decreased sperm motility [19].
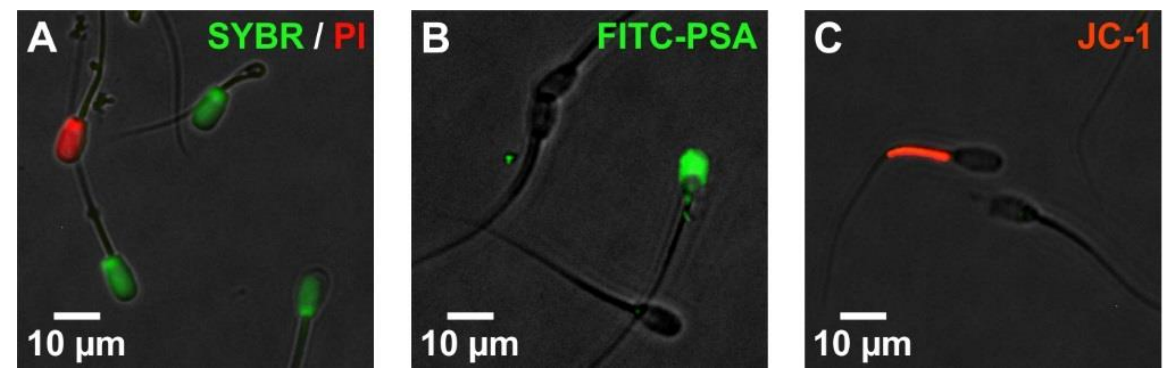

Fig. 7-3: Investigation of sperm quality using fluorescent staining. A) The plasma membrane was investigated using SYBR/PI, yielding green and red fluorescence for intact and damaged membranes, respectively. B) The integrity of the acrosome was studied using FITC-PSA, staining damaged acrosomes using FITC. C) JC-1 was used to investigate the mitochondrial membrane potential. Mitochondrial membranes with a high potential were stained orange/red, whereas depolarized membranes were stained green or remained unstained. 


\subsubsection{Induced cell damage}

Potential cell damage, inflicted by DEP excitation, was investigated by studying the integrity of the plasma and acrosomal membrane (Fig. 7-3A and B, respectively) and the membrane potential of the mitochondrial membrane (Fig. 7-3C). The effect of DEP excitation at $10 \mathrm{MHz}$ was investigated at a potential of 3 and $6 \mathrm{~V}$ (Fig. 7-4). In each experiment, a strict protocol was followed to minimize cell damage by confounding factors. Each experiment consisted of an off-chip control, on-chip control ( $0 \mathrm{~V}$ ) and on-chip experiment (3 or $6 \mathrm{~V})$, at which sample was collected to asses all three quality characteristics. The results are shown in Fig. 7-4. Experiments using $3 \mathrm{~V}$ and $6 \mathrm{~V}$ excitation were performed 6 and 4 times, respectively. The offand on-chip controls of experiments at both potentials were combined, yielding 10 off-chip experiments and 7 or 8 on-chip experiments per staining.

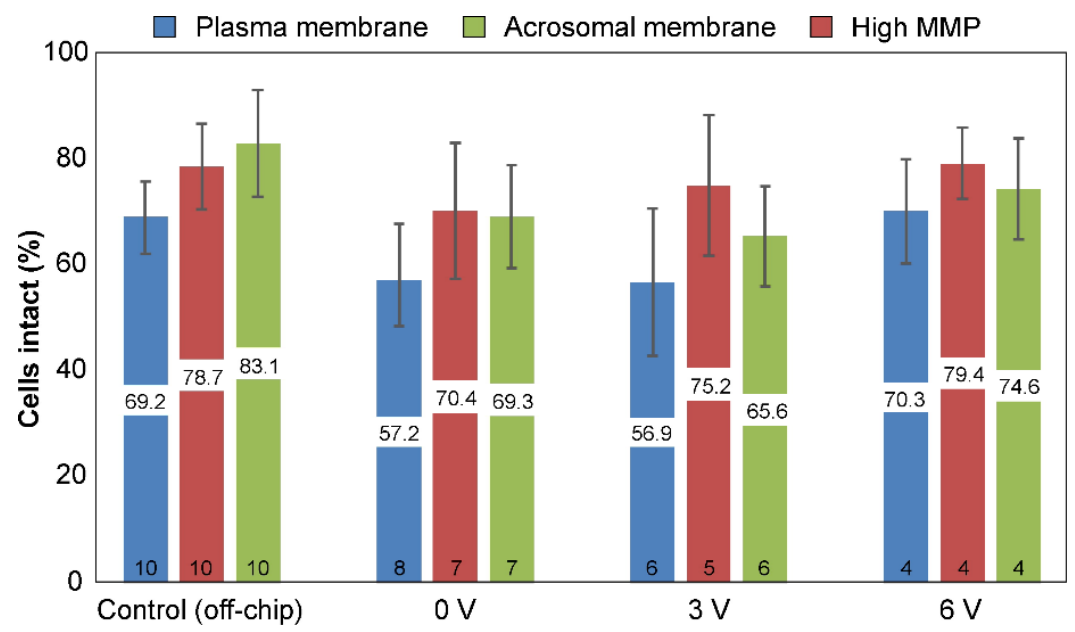

Fig. 7-4: Effect of DEP on sperm quality at $10 \mathrm{MHz}$. No significant differences were observed between sperm quality after DEP manipulation at 3 or $6 \mathrm{~V}$ compared to the on-chip control $(0 \mathrm{~V})$. This implies that no cell damage is caused by short-term exposing the sperm cells to a strong electrical field. A small decrease in sperm quality (of all parameters) was observed between the on-chip controls and the offchip controls. This suggests that sperm injection and handling on-chip resulted in slight sperm damage (mean values and \# of experiments performed are shown in the centre and the base of the bar graphs, respectively; error bars $=1 x$ standard deviation). 

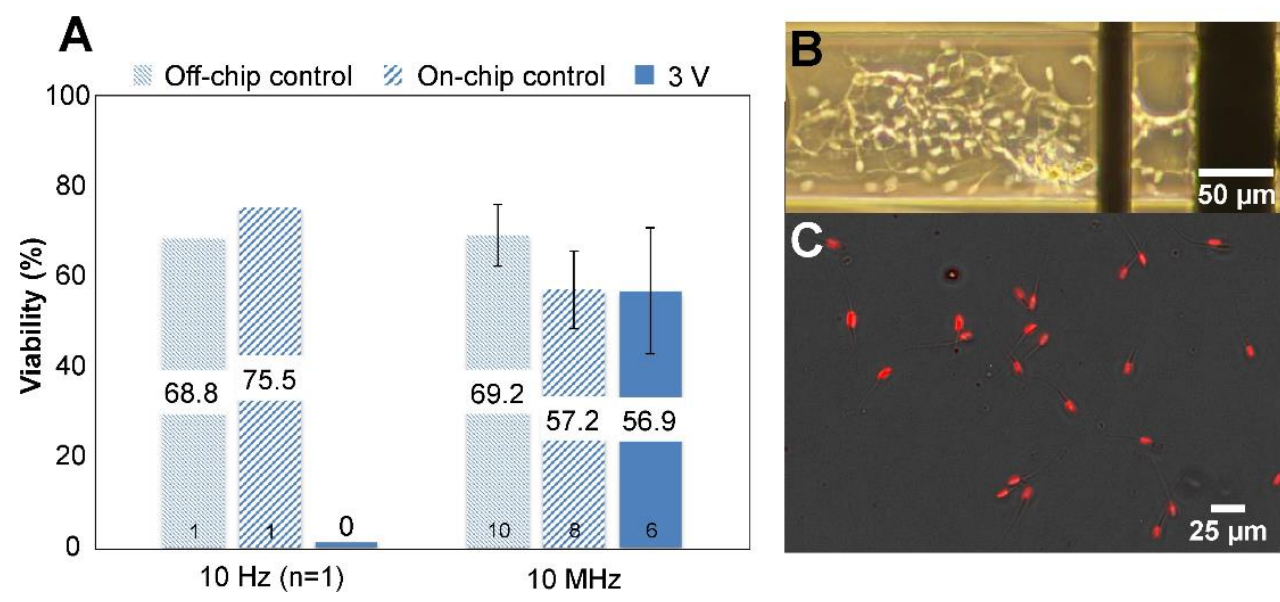

Fig. 7-5: Effect of DEP on sperm viability at a low frequency (10 $\mathrm{Hz}$ ). A) DEP excitation at $10 \mathrm{~Hz}$ resulted in a complete loss of cell viability, resulting in $\mathrm{B}$ ) cell clogging in the microfluidic channel and C) $100 \%$ positive PI staining after cell collection (mean values and \# of experiments performed are shown in the centre and the base of the bar graphs respectively; error bars $=1 \mathrm{x}$ standard deviation).

No significant effect of DEP was observed on the integrity of the plasma, acrosomal and mitochondrial membranes when comparing the results at both potentials with on-chip control experiments at both potentials. When comparing the off-chip control to the on-chip electrical experiments, a slight decrease can be observed in the amount of cells with intact membranes. This decrease was likely caused by sperm handling outside the chip and sperm injection and handling on-chip.

Exposing sperm cells to a $300 \mathrm{kV} \cdot \mathrm{m}^{-1}(6 \mathrm{~V}), 10 \mathrm{MHz}$ electrical field did not result in significant cell damage. According to the simulation of the TMP, at high frequencies the induced potential is low, inflicting relative low cell damage. However, at lower frequencies $(<1 \mathrm{MHz}$ ) higher induced TMPs are expected, resulting in increased cell damage. To investigate the effect of low frequency excitation, sperm cells were exposed to a $10 \mathrm{~Hz} 3 \mathrm{~V}$ electrical field. A drastic effect of $10 \mathrm{~Hz}$ excitation was observed on the sperm viability (Fig. 7-5A). After the experiment, a high number of sperm cells were stuck to the channel walls and electrodes (Fig. 7-5B) and all collected cells stained positive for PI, indicating $100 \%$ cell death (Fig. 7-5C). These findings are consisted with the simulated TMP, which predicts cell damage at low frequency excitation. The chip could not be used for further experimentation, since 
the cells were solidly stuck to the channel walls and could not be removed after the experiment.

\subsection{Conclusions}

The use of DEP is an interesting approach for microfluidic sperm manipulation and selection. However, DEP can inflict cell damage by inducing high transmembrane potentials and/or by inducing high fluid temperatures by Joule heating [9]. Simulation of the induced TMP in the microfluidic device was performed to study its impact on the sperm cell quality. This simulation showed a high TMP at low frequencies, which is described as harmful in literature [9]. At frequencies $>10$ $\mathrm{MHz}$, the induced potential dropped significantly, suggesting relative low cell damage at high frequencies. This was confirmed by experiments performed at 10 $\mathrm{MHz}$ at 3 or $6 \mathrm{~V}$ potential. These experiments showed no effect of DEP on the integrity of the plasma and acrosomal membrane and no decrease in mitochondrial membrane potential was observed. However, a slight decrease in cell quality was observed between on-chip and off-chip control experiments, which can potentially be prevented by optimizing sperm handling, injection and collection methods. However, DEP experiments at $10 \mathrm{~Hz}$ showed a dramatic effect on the cell viability. These findings are consistent with predictions based on the simulation of the transmembrane potential. Concludingly, the presented results showed that 10 $\mathrm{MHz}$ DEP excitation did not negatively affect the sperm quality. These findings are the first to report the non-invasive nature of DEP as a tool for sperm manipulation in microfluidic systems.

\subsection{Acknowledgements}

Financial support from the NWO - Netherlands Organization of Scientific Research (Spinoza Grant A. van den Berg, Veni L.I. Segerink), the technical support of F. van Rossem, P.M. ter Braak, J.W. van Nieuwkasteele, H.L. de Boer and J.G. Bomer are gratefully acknowledged. We also thank the "KI Twenthe" for the kind supply of boar semen samples. 


\subsection{References}

1. Knowlton, S.M., M. Sadasivam, and S. Tasoglu, Microfluidics for sperm research. Trends Biotechnol, 2015. 33(4): p. 221-9.

2. Said, T.M. and J.A. Land, Effects of advanced selection methods on sperm quality and ART outcome: a systematic review. Hum Reprod Update, 2011. 17(6): p. 719-33.

3. Cho, B.S., et al., Passively driven integrated microfluidic system for separation of motile sperm. Anal Chem, 2003. 75(7): p. 1671-5.

4. Seo, D.B., et al., Development of sorting, aligning, and orienting motile sperm using microfluidic device operated by hydrostatic pressure. Microfluid and Nanofluid, 2007. 3(5): p. 561-70.

5. Lopez-Garcia, M.D.C., et al., Sperm motion in a microfluidic fertilization device. Biomedic Microdevices, 2008. 10(5): p. 709-18.

6. Qiu, T., et al. A microfluidic "treadmill" for sperm selective trapping according to motility classification. in Solid-State Sensors, Actuators and Microsystems Conference (transducers), 2011 16th International. 2011.

7. Chen, Y.A., et al., Analysis of sperm concentration and motility in a microfluidic device. Microfluid Nanofluid, 2011. 10(1): p. 59-67.

8. Gagnon, Z.R., Cellular dielectrophoresis: Applications to the characterization, manipulation, separation and patterning of cells. Electrophoresis, 2011. 32(18): p. 2466-87.

9. Voldman, J., Dielectrophoretic Traps for Cell Manipulation, in BioMEMS and Biomedical Nanotechnology, M. Ferrari, R. Bashir, and S. Wereley, Editors. 2007, Springer US. p. 159-86.

10. Fuhr, G., et al., High-frequency electric field trapping of individual human sperm cells. Hum Reprod, 1998. 13(1): p. 136-41.

11. Rosales-Cruzaley, E., et al., Sperm cells manipulation employing dielectrophoresis. Bioproc Biosyst Eng, 2013. 36(10): p. 1353-62.

12. Silva, P.F.N. and B.M. Gadella, Detection of damage in mammalian sperm cells. Theriogenology, 2006. 65(5): p. 958-78.

13. Kenausis, G.L., et al., Poly(L-lysine)-g-poly(ethylene glycol) layers on metal oxide surfaces: Attachment mechanism and effects of polymer architecture on resistance to protein adsorption. J Phys Chem-B, 2000. 104(14): p. 3298309.

14. Hossain, M.S., et al., Flow cytometry for the assessment of animal sperm integrity and functionality: state of the art. Asian J Androl, 2011. 13(3): p. 406-19. 
15. Amaral, A. and J. Ramalho-Santos, Assessment of mitochondrial potential: implications for the correct monitoring of human sperm function. Int $\mathrm{J}$ Androl, 2010. 33(1): p. e180-6.

16. Pauly, H. and H.P. Schwan, Uber die Impedanz einer Suspension von kugelförmigen Teilchen mit einer Schale. Z. Naturforsch, 1959. 14b(125): $p$. 125-31.

17. Saravia, F., et al., Differences in boar sperm head shape and dimensions recorded by computer-assisted sperm morphometry are not related to chromatin integrity. Theriogenology, 2007. 68(2): p. 196-203.

18. COMSOL 4.4. Introduction to Heat Transfer Module. 2013; Available from: https://www.comsol.com/heat-transfer-module.

19. Paoli, D., et al., Mitochondrial membrane potential profile and its correlation with increasing sperm motility. Fertil Steril, 2011. 95(7): p. 23159.

20. Erdem, A., et al., The Effect of Swim-up and Gradient Sperm Preparation Techniques on Deoxyribinucleic Acid (DNA) Fragmentation in Subfertile Patients. Fertil Steril, 2013. 100(3): S453.

21. Natali, I., Sperm Preparation Techniques for Artificial Insemination Comparison of Sperm Washing, Swim Up, and Density Gradient Centrifugation Methods, in Artificial Insemination in Farm Animals, M. Manafi, Editor. 2011, InTech. 


\section{Summary and outlook}

In this chapter, a summary of the main results and conclusions is presented. Subsequently, recommendations and potential research directions are discussed in the outlook.

\subsection{Summary}

Microfluidic technology has been occasionally used for the analysis and separation of sperm cells. Multiple reports have shown the ability to study sperm motility in microfluidic channels and to sort motile sperm cells in passively driven microfluidic systems. Besides population-based approaches for sperm selection, non-invasive analysis of individual sperm cells by electrical methods and the ability to select/sort them individually, has potential for sperm selection for ART procedures and for sperm refinement applications in the livestock industries.

For many applications, individual cells have to be confined to a fixed position during cell analysis. A big variety of methods are available to temporarily entrap individual cells using hydrodynamic, electrical, chemical, optical, acoustic and magnetic methods (chapter 2). Each of these methods has its own advantages and disadvantages. One of the aims, described in this thesis, is to perform electrical analysis on individual entrapped sperm cells. Therefore, a table of requirements is constructed to find the most suitable trapping method for our application. The trapping method must allow stable, reversible and non-invasive trapping of sperm cells and must allow integration with the microelectrodes required for electrical analysis. Based on these requirements, hydrodynamic trapping is considered as most suitable for trapping of individual sperm cells.

In general, electrical analysis of cells in microfluidic systems is performed by measuring changes in impedance when a cell is introduced in between two microelectrodes (chapter 3 ). Based on this difference, information regarding cell size, membrane and/or cytoplasm can be extracted. However, the microfluidic system itself has an influence on the measured electrical response. Primarily, at low frequencies the impedance of the total setup is greatly influenced by the double layer capacitance, whereas at high frequencies, the impedance is mainly determined by parasitic effects of the system. The best measurement frequency can be found at intermediate frequencies, at which the total impedance of the system is mainly determined by the electrolyte resistance. The design of an 
equivalent circuit model may help in predicting the electrical response of a setup and to find the optimal measurement frequency.

Two techniques, which are used abundantly for the analysis of single cells in microfluidic systems, are microfluidic impedance cytometry and microfluidic electrical impedance spectroscopy. In microfluidic impedance cytometry, cells are flown through a microfluidic channel with at least one electrode pair to record changes in impedance using at least one measurement frequency. Examples of applications are the discrimination of cell types from cell populations and the analysis of cell viability, morphology and differentiation. In microfluidic electrical impedance spectroscopy, typically, a cell is entrapped while the impedance is monitored over a broad frequency range. Using this technique, researchers have studied the electrical properties of cancer cells and have investigated the effects of toxin/drug exposure and ion channel blockage on the electrical properties of cells.

Development of a microfluidic system for the hydrodynamic entrapment of individual sperm cells is reported in chapter 4. Sperm cells were entrapped individually in small microfluidic side channels, which connect two larger main channels. By inducing a pressure difference between the main channels, fluid flow through the side channels dragged the sperm cells towards the side channels and trapped them at the channel entrance. Side channels with a height of $1 \mu \mathrm{m}$ were most suitable for single sperm cell entrapment. After entrapment, this platform was used to analyse the sperm plasma membrane, acrosomal membrane and sexchromosome content using fluorescent microscopy.

The previously described trapping platform was integrated with microelectrodes to perform electrical analysis of the motility of single spermatozoa (chapter 5). Individual sperm cells were entrapped while recording the impedance differentially and continuously at $1 \mathrm{MHz}$. After data recording, Fast Fourier Transform analysis showed a consistent base frequency in the signal caused by sperm beating in between the electrodes. This observed frequency showed high correlation with the sperm beat frequency determined by optical analysis. To test the robustness of the electrical analysis method, the sperm motility was altered by changing the temperature and exposing entrapped sperm cells to caffeine. In both situations, the sperm beat frequency was altered, which was electrically characterized.

Besides performing sperm analysis under static conditions, a microfluidic platform was designed and fabricated to perform microfluidic impedance cytometry at which sperm cells were flown through a pair of microelectrodes while recording the impedance (chapter 6 ). This approach was used to investigate cytoplasmic droplet 
content, which is a commonly occurring sperm defect in the livestock applications. When flowing sperm cells through a small microfluidic channel and recording the impedance differentially at $1.3 \mathrm{MHz}$, the resulting impedance showed a distinct effect of the sperm head and tail.

Using the shape of the impedance signal over time, the orientation of the sperm cell could be determined. When a cytoplasmic droplet was present on the sperm flagellum, an additional blob was observed on this impedance signal, which was analysed using the area under the curve. After normalization of this area, a significant difference was observed between a population of cells with and without sperm cells with cytoplasmic droplets on the tail. A receiver operating characteristic curve was plotted and yielded an area under the curve of 0.85 .

Additionally, a label-free cell sorting system is described, which is used to sort cells by dielectrophoresis based on recorded changes in impedance (chapter 6 ). This sorting system included three separate features: focusing, impedance detection and sorting. Firstly, sperm cells were focused using dielectrophoresis using a 4 electrode system. By exciting the focusing electrodes using a $10 \mathrm{MHz} 3 \mathrm{~V}$ sinusoidal signal, the sperm cells were pushed towards the middle of the channel, resulting in a more uniform cell location and velocity. Secondly, changes in impedance were recorded using a single electrode pair. In a proof of concept experiment, a mixture of $3 \mu \mathrm{m}$ beads and sperm cells were infused through the chip while recording changes in impedance. Based on differences between the impedance change of beads compared to sperm cells, beads could be selectively sorted to a separate channel using dielectrophoresis by actively controlling the excitation of the sorting electrodes. This sorting system might be a potential tool towards microfluidic sperm refinement.

Manipulation of sperm cells by dielectrophoresis is a relatively unexplored area. To investigate whether sperm cells can withstand the electrical fields, the sperm quality was investigated after exposing cells to electrical fields on-chip (chapter 7). The sperm quality was assessed by investigating the integrity of the plasma and acrosomal membranes and by analysis of the mitochondrial membrane potential. Electrical fields at high frequency $(10 \mathrm{MHz})$ did not result in induced cell damage, although some cell damage was observed due to sperm handling, injection and collection on-chip. When reducing the field frequency to near $D C(10 \mathrm{~Hz})$, extensive sperm damage was observed. These findings were consistent with the simulation of the transmembrane potential, which showed a high induced transmembrane potential at low frequencies. 


\subsection{Outlook}

This thesis presented upon the design and fabrication of two separate platforms for single sperm analysis. The first microfluidic platform was used to perform electrical analysis of the beat frequency of individually entrapped sperm cells. This platform could be a potential tool for advanced sperm selection for ICSI treatments in the clinic.

Currently, this platform is not suitable for sperm selection applications. Retrieving a single sperm cell using a microneedle, which is typically used for single sperm selection and injection into an egg cell during ICSI treatments, can be very challenging because the cell traps are situated in a closed channel environment. Furthermore, the amount of traps in the current device is limited. A potential approach to design a new platform, which can improve sperm selection in the clinic, should ensure that the current methods for sperm selection can still be used in the microfluidic platform. Currently, sperm selection is performed by putting a drop of diluted sample on a pre-warmed substrate. Since sperm cells have a tendency to swim close to surfaces, the motile sperm cells swim close to the substrate surface at the outer edge of the drop. Sperm selection for ICSI is typically performed by isolating a single, motile sperm cell at this outer edge. The microfluidic platform can be designed in such a way that the trapping channels envelop an open, circular well in which a sample is introduced (Fig. 8-1).

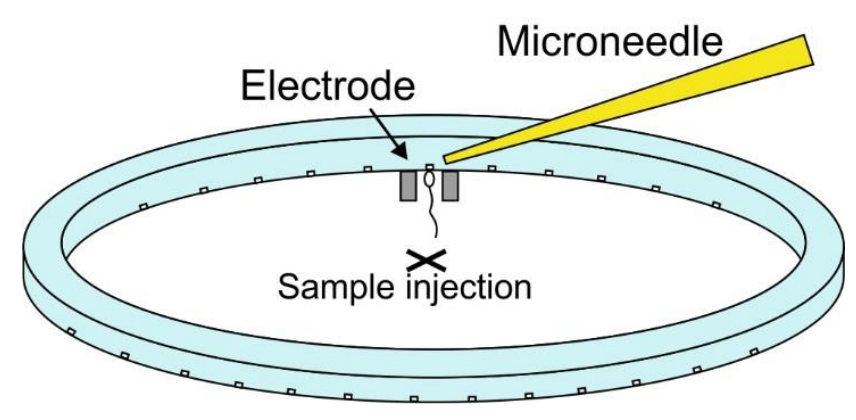

Fig. 8-1: Sketch of a microfluidic platform design for analysis of single sperm motility and sperm selection.

The motile sperm cells swim towards the outer edge of the well and are trapped in the trapping channels. After electrical analysis, the sperm cell with the highest motility can be isolated using a conventional microneedle. 
Although sperm motility is an important characteristic for the sperm selection, it does not solely predict fertilization outcome after ICSI treatment. Ramos et al. [1] showed that no more than $55 \%$ of the selected sperm for ICSI procedures had intact DNA. Therefore, a combined approach of sperm analysis methods might be necessary to improve sperm selection and ICSI outcome. An example of a relative new method for sperm selection is the use of hyaluronic acid (HA) [2]. Mature sperm cells develop HA-binding sites on their membrane, which have been used as a parameter for sperm selection. Although this technique has not been fully evaluated [2], some reports show the isolation of sperm with high DNA integrity using HA-binding [3, 4]. A combination of single sperm motility analysis with HAbinding might be an interesting approach.

The second microfluidic platform was used to distinguish cytoplasmic droplet content on the sperm tail and showed the ability to perform label-free impedance activated sorting of beads and sperm cells by dielectrophoresis. This approach could be valuable for sperm refinement in the livestock industry.

Using microfluidic impedance analysis, cytoplasmic droplet content was investigated. Potentially, other morphological defects can be investigated using the same platform, including double heads and tail defects. Furthermore, reports in literature show the use of impedance cytometry for the analysis of cell viability [5], membrane properties [6] and the cell interior $[7,8]$ using multi frequency analysis. Therefore, impedance cytometry should be studied in more detail to investigate its potential to analyse sperm properties such as sperm cell viability, acrosome integrity and internal environment. Correspondingly, the device design may be optimized to improve cytometric analysis.

A similar platform was designed to perform non-invasive impedance activated cell sorting. In a proof-of-concept experiment, $3 \mu \mathrm{m}$ beads and sperm cells were sorted by dielectrophoresis based on their recorded impedance. The sorting speed is currently limited to the computational speed of the custom-built LabVIEW program. In order to achieve the required throughput for sperm refinement applications in the livestock industry, which is in the order of $1000 \mathrm{~s}$ of cells $\cdot \mathrm{s}^{-1}, 10$ 100 multiplexed channels are required with analogue control systems to achieve this sorting speed. Furthermore, impedance analysis of sperm cells can be improved by reducing the measurement volume, implementing differential electrode pairs in the sorting chip and by adding additional shielding electrodes to the current design. 


\subsection{References}

1. Ramos, L., et al., Evaluation of ICSI-Selected epididymal sperm samples of obstructive azoospermic males by the CKIA system. J Androl, 2004. 25(3): $p$. 406-11.

2. Said, T.M. and J.A. Land, Effects of advanced selection methods on sperm quality and ART outcome: a systematic review. Hum Reprod Update, 2011. 17(6): p. 719-33.

3. Parmegiani, L., et al., "Physiologic ICSI": Hyaluronic acid (HA) favors selection of spermatozoa without DNA fragmentation and with normal nucleus, resulting in improvement of embryo quality. Fertil Steril, 2010. 93(2): p. 598-604.

4. Parmegiani, L., et al., Efficiency of hyaluronic acid (HA) sperm selection. J Assist Reprod Gen, 2010. 27(1): p. 13-6.

5. Mernier, G., et al., Cell viability assessment by flow cytometry using yeast as cell model. Sensor Actuat B-Chem, 2011. 154(2): p. 160-3.

6. David, F., et al., Viability and membrane potential analysis of Bacillus megaterium cells by impedance flow cytometry. Biotechnol Bioeng, 2012. 109(2): p. 483-92.

7. Kuttel, C., et al., Label-free detection of Babesia bovis infected red blood cells using impedance spectroscopy on a microfabricated flow cytometer. Acta Trop, 2007. 102(1): p. 63-8.

8. Zhao, Y., et al., Tumor cell characterization and classification based on cellular specific membrane capacitance and cytoplasm conductivity. Biosens Bioelectron, 2014. 57: p. 245-53. 


\section{Appendix A - Fabrication of glass chips}

\section{A.1 Description}

In this appendix, the fabrication of glass microchips, used in experiments described in chapter 6 and 7, is summarized. A cross-section of these devices is shown in fig. A.1. In short, microelectrodes were embedded in 500 and $1100 \mu \mathrm{m}$ thick Borofloat wafers after wet-etching. Access holes for electrical and fluidic connection where created by powderblasting. Subsequently, microfluidic features were developed in a $20 \mu \mathrm{m}$ thick foil, which was sandwiched in between two Borofloat wafers after development and alignment. After bonding, the chips were diced to the right dimensions.

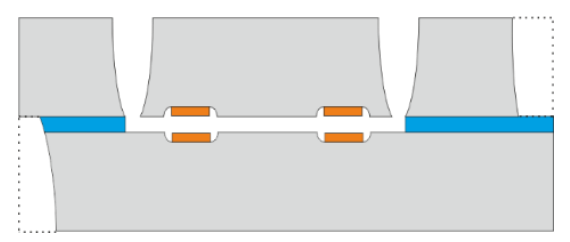

Fig. A.1: Cross-section of two borofloat substrates (grey) with embedded Tantalum-Platinum electrodes (orange) and powderblasted access holes that sandwich a layer of PerMX3020 foil (blue, $20 \mu \mathrm{m}$ thick), which contains microfluidic channel features. 


\section{A.2 Mask overview}

\begin{tabular}{|l|l|l|l|l|}
\hline Mask & Wafer & Layer & Inside white/black & Mirrored \\
\hline Mask 1 - PerMX3020 & Bottom & L1 & IB & No \\
\hline Mask 2 - Electrodes & Bottom & L12 & IW & No \\
\hline Mask 3 - Electrodes & Top & L13 & IW & Yes \\
\hline Mask 4 - Powderblast & Bottom & L4 & IB & Yes \\
\hline Mask 5 - Powderblast & Top & L5 & IB & No \\
\hline
\end{tabular}

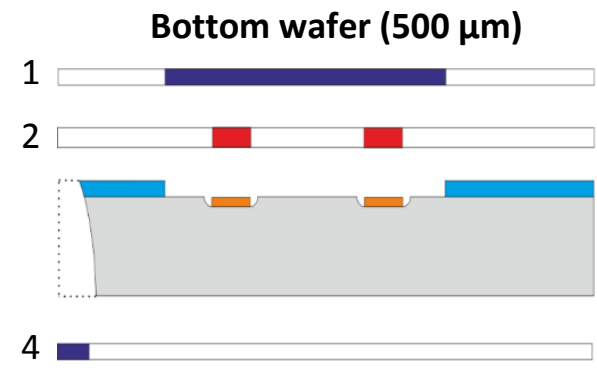

Top wafer $(1100 \mu \mathrm{m})$

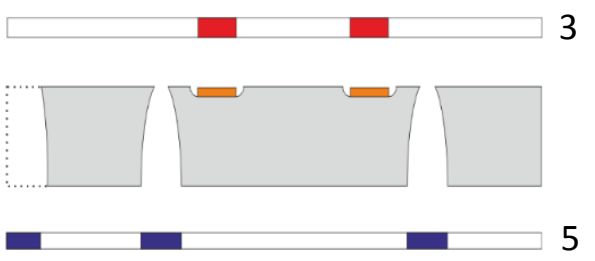

Fig. A.2: Cross-section of the bottom $(500 \mu \mathrm{m})$ and the top BF33 wafer $(1100 \mu \mathrm{m})$ including the required masks for fabrication. 


\section{A.3 Mask and chip layout}

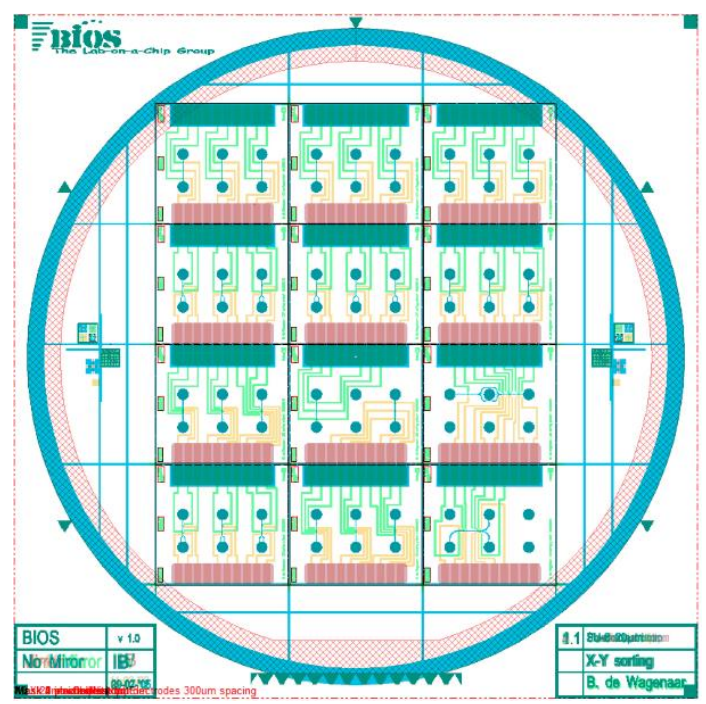

Fig. A.3: Total wafer design including mask 1 for the fluidic features, masks 2 and 3 for microelectrode fabrication and masks 4 and 5 for powderblasting electric and fluidic inlets.
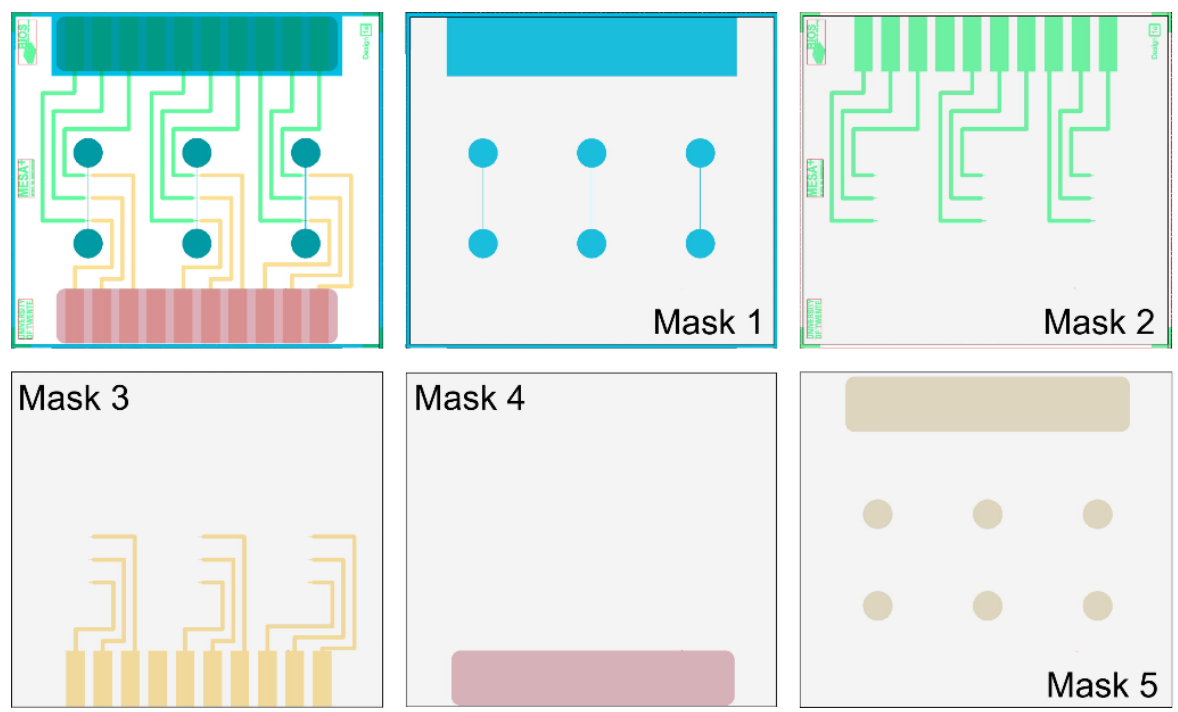

Fig. A.4: Zoom-in on the design of a single chip, including 5 separate masks. 


\section{A.4 Mask alignment}

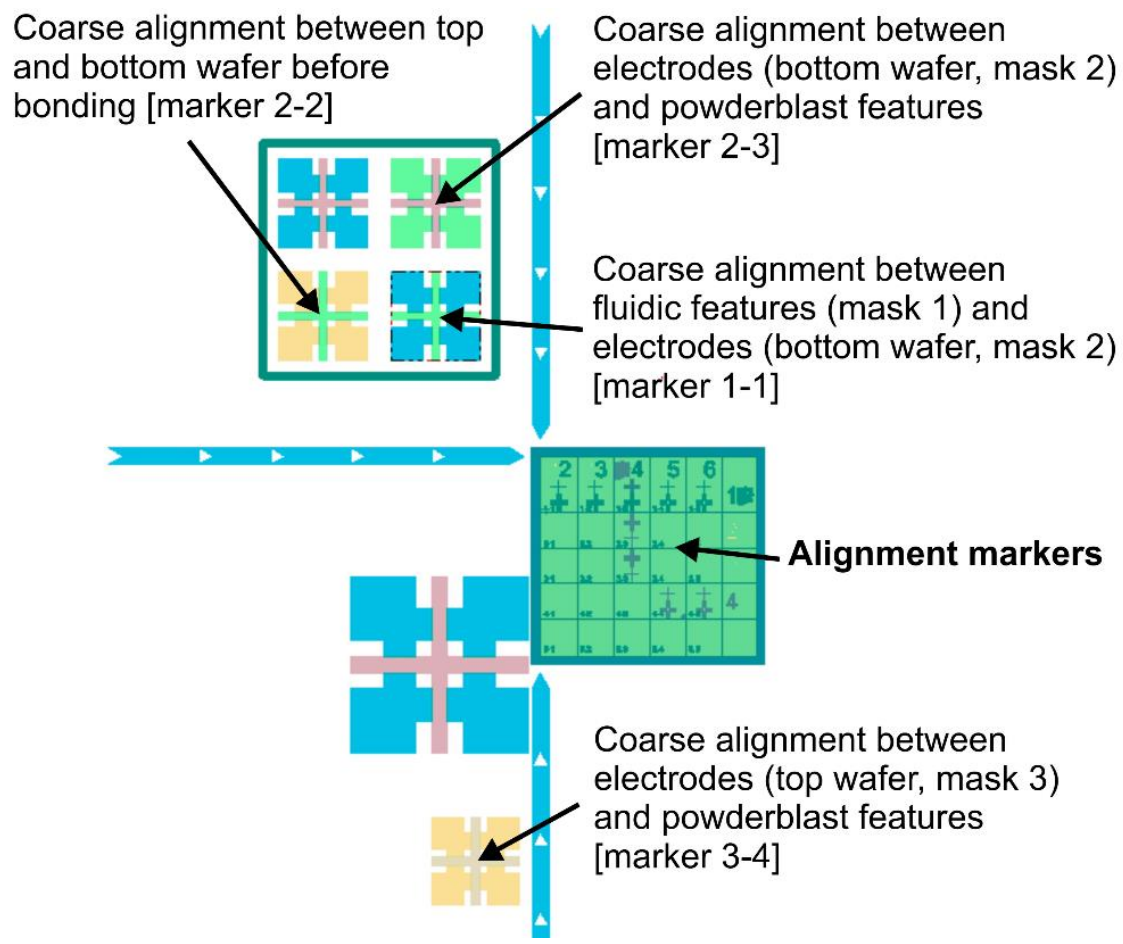

Fig. A.5: Wafers and masks were aligned roughly by coarse alignment and precisely using dedicated alignment markers.
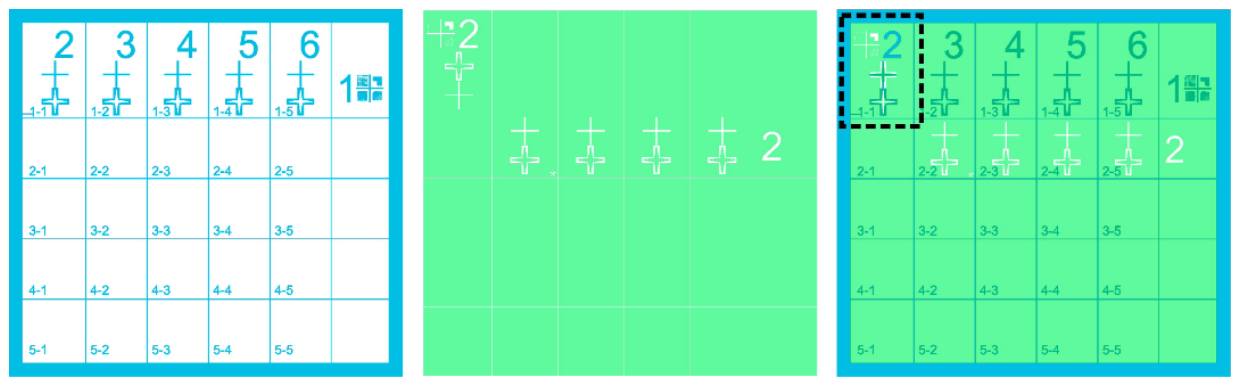

Fig. A.6: Example of alignment using alignment markers. The fabricated microelectrodes (mask 2, green) on the wafer are aligned to the alignment markers on the mask 1 (blue) to align the fluidic features with respect to the microelectrodes. In this example, marker 1-1 is used for alignment. 


\section{A.5 Fabrication process flow}

Top wafer $(1100 \mu \mathrm{m})$

\begin{tabular}{|c|c|c|c|}
\hline & \multicolumn{2}{|l|}{ Process description } & \multirow[t]{2}{*}{ Cross-section } \\
\hline 1 & Select substrate & - $1100 \mu \mathrm{m}$ Borofloat 33 & \\
\hline 2 & \multirow{4}{*}{$\begin{array}{l}\text { Standard cleaning } \\
\text { Wetbench } 16\end{array}$} & - $99 \% \mathrm{HNO}_{3}$ (beaker 1, $5 \mathrm{~min}$ ) & \\
\hline 3 & & - $99 \% \mathrm{HNO}_{3}$ (beaker 2, $5 \mathrm{~min}$ ) & \\
\hline 4 & & $\begin{array}{l}\text { - Quick dump rinse (DI resistivity } \\
>10.5 \Omega \mathrm{M})\end{array}$ & \\
\hline 5 & & $\begin{array}{l}\text { - Single wafer spin drying } \\
\text { (2500 rpm, 60s) }\end{array}$ & \\
\hline & \multirow{13}{*}{$\begin{array}{l}\text { Photolithography } \\
\text { Wetbench } 21 \\
\text { EVG620 Mask } \\
\text { Aligner }\end{array}$} & Resist & \\
\hline 6 & & - Spin HDMS (4000 rpm, 30s) & \\
\hline 7 & & $\begin{array}{l}\text { - Spin Olin Oir 907-17 } \\
\text { (4000 rpm, 30s) }\end{array}$ & \\
\hline 8 & & - Prebake $\left(95^{\circ} \mathrm{C}, 60 \mathrm{~s}\right)$ & \\
\hline & & Exposure & \\
\hline \multirow{4}{*}{9} & & $\begin{array}{l}\text { - Exposure: Hg-lamp } \\
\left(12 \mathrm{~mW} / \mathrm{cm}^{2}, 4 \mathrm{~s}\right)\end{array}$ & \\
\hline & & - Top side, hard-contact & \\
\hline & & - Mask 3 & \\
\hline & & Development & \\
\hline 10 & & $\begin{array}{l}\text { - OPD4262 (beaker } 1 \text { - 45s, } \\
\text { beaker } 2 \text { - 15s) }\end{array}$ & \\
\hline 11 & & - Quick dump rinse & \\
\hline 12 & & - Single wafer spin drying & \\
\hline 13 & & - Post-bake $\left(120^{\circ} \mathrm{C}, 10 \mathrm{~min}\right)$ & \\
\hline 14 & \multirow{4}{*}{$\begin{array}{l}\text { BHF wet etching } \\
\text { Wetbench } 9 / 10\end{array}$} & - UV-Ozone cleaning (5 min) & \\
\hline 15 & & $\begin{array}{l}\text { - BHF wet etch } \\
\text { (etch rate } 0.5 \mathrm{~nm} / \mathrm{s}, 6 \mathrm{~min} \text { ) }\end{array}$ & \\
\hline 16 & & - Quick dump rinse & \\
\hline 17 & & - Single wafer spin dryer & \\
\hline 18 & $\begin{array}{l}\text { Sputtering Ta-Pt } \\
\text { Sputterke }\end{array}$ & $\begin{array}{l}\text { - Sputter Ta } \\
\text { (deposition rate } 16.5 \mathrm{~nm} / \mathrm{min} \text { ) } \\
\text { Deposition time for } 30 \mathrm{~nm}=109 \mathrm{~s} \\
\text { after } 1 \text { min pre-sputter. }\end{array}$ & \\
\hline
\end{tabular}




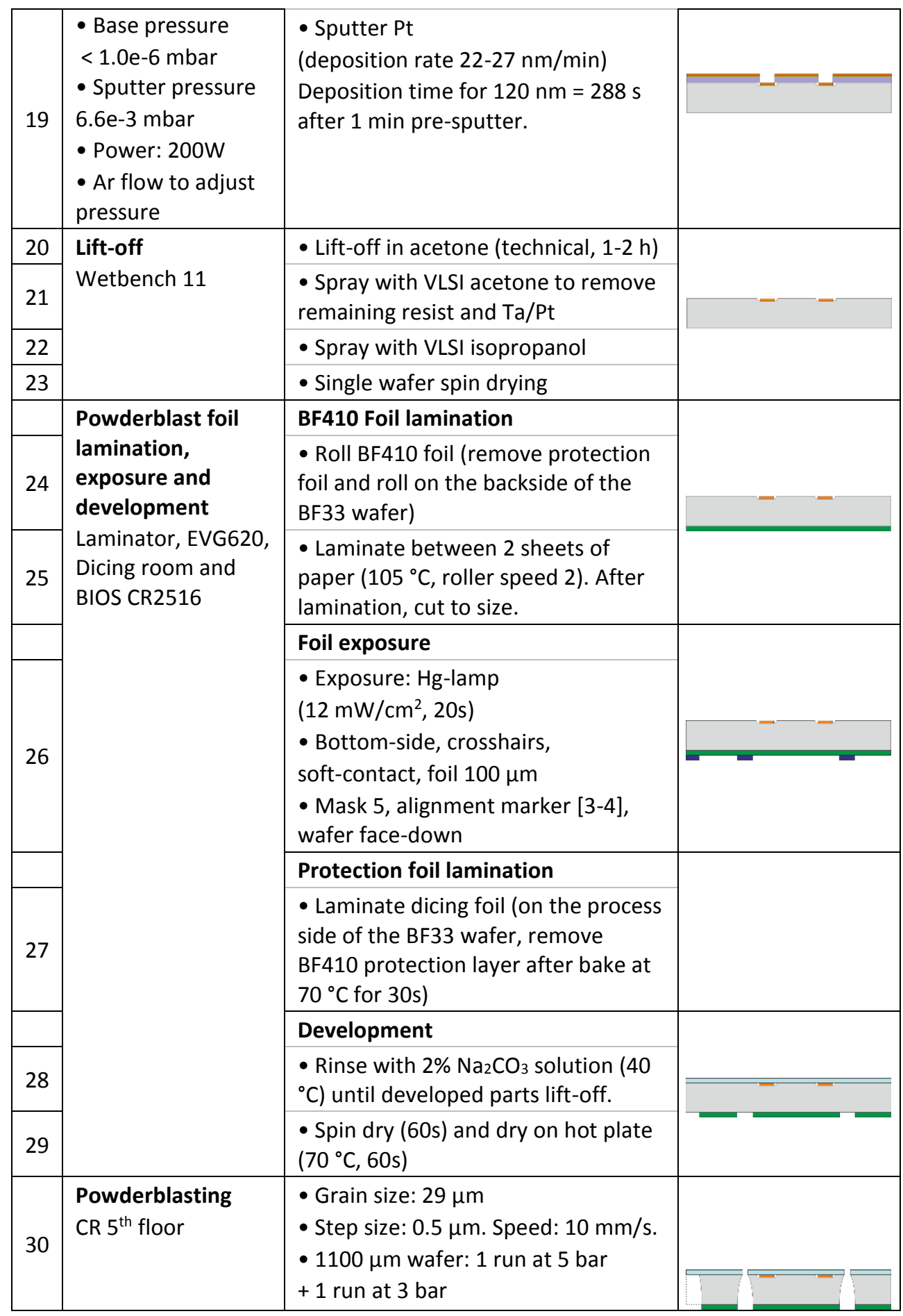




\begin{tabular}{|c|c|c|c|}
\hline & & $\begin{array}{l}\text { or } 2 \text { runs at } 5 \text { bar with } 2 \text { layers of } \\
\text { BF410 foil }\end{array}$ & \\
\hline 31 & \multirow[t]{2}{*}{$\begin{array}{l}\text { Delamination and } \\
\text { cleaning } \\
\text { BIOS CR2615 }\end{array}$} & $\begin{array}{l}\text { - Remove BF410 and dicing foil by } \\
\text { immersing in warm water }\left( \pm 40^{\circ} \mathrm{C}\right) \text {. } \\
\text { Peel foils in parallel with respect to } \\
\text { the wafer's surface to prevent } \\
\text { breaking. }\end{array}$ & \\
\hline 32 & & $\begin{array}{l}\text { - Remove particles by ultrasound in } \\
\text { DI ( } 30 \mathrm{~min})\end{array}$ & \\
\hline 33 & \multirow{3}{*}{$\begin{array}{l}\text { Removal of } \\
\text { particles } \\
\text { Wetbench } 11\end{array}$} & $\begin{array}{l}\text { - Dipping in } 99 \% \mathrm{HNO}_{3} \\
\text { (beaker 1, 20s-30s) }\end{array}$ & \\
\hline 34 & & - Quick dump rinse & \\
\hline 35 & & - Single wafer spin drying & \\
\hline
\end{tabular}

\section{Bottom wafer $(500 \mu \mathrm{m})$}

\begin{tabular}{|c|c|c|c|}
\hline & \multicolumn{2}{|l|}{ Process description } & \multirow[t]{2}{*}{ Cross-section } \\
\hline 1 & Select substrate & - $500 \mu \mathrm{m}$ Borofloat 33 & \\
\hline 2 & \multirow{4}{*}{$\begin{array}{l}\text { Standard cleaning } \\
\text { Wetbench } 16\end{array}$} & - $99 \% \mathrm{HNO}_{3}$ (beaker 1, 5 min) & \\
\hline 3 & & - $99 \% \mathrm{HNO}_{3}$ (beaker 2, $5 \mathrm{~min}$ ) & \\
\hline 4 & & $\begin{array}{l}\text { - Quick dump rinse } \\
\text { (DI resistivity }>10.5 \Omega \mathrm{M} \text { ) }\end{array}$ & \\
\hline 5 & & $\begin{array}{l}\text { - Single wafer spin drying } \\
\text { (2500 rpm, 60s) }\end{array}$ & \\
\hline & \multirow{11}{*}{$\begin{array}{l}\text { Photolithography } \\
\text { Wetbench } 21 \text { and } \\
\text { EVG620 Mask } \\
\text { Aligner }\end{array}$} & Resist & \\
\hline 6 & & - Spin HDMS (4000 rpm, 30s) & \\
\hline 7 & & $\begin{array}{l}\text { - Spin Olin Oir 907-17 } \\
\text { (4000 rpm, 30s) }\end{array}$ & \\
\hline 8 & & - Prebake $\left(95^{\circ} \mathrm{C}, 60 \mathrm{~s}\right)$ & \\
\hline & & Exposure & \\
\hline \multirow{4}{*}{9} & & $\begin{array}{l}\text { - Exposure: Hg-lamp } \\
\left(12 \mathrm{~mW} / \mathrm{cm}^{2}, 4 \mathrm{~s}\right)\end{array}$ & \\
\hline & & - Top-side, hard-contact & \\
\hline & & - Mask 2 & \\
\hline & & Development & \\
\hline 10 & & $\begin{array}{l}\text { - OPD4262 (beaker } 1 \text { - 45s, } \\
\text { beaker } 2 \text { - 15s) }\end{array}$ & \\
\hline 11 & & - Quick dump rinse & \\
\hline
\end{tabular}




\begin{tabular}{|c|c|c|c|}
\hline 12 & & - Single wafer spin drying & \\
\hline 13 & & - Post-bake $\left(120^{\circ} \mathrm{C}, 10 \mathrm{~min}\right)$ & \\
\hline 14 & \multirow{4}{*}{$\begin{array}{l}\text { BHF wet etching } \\
\text { Wetbench } 9 / 10\end{array}$} & - UV-Ozone cleaning (5 min) & \\
\hline 15 & & $\begin{array}{l}\text { - BHF wet etch } \\
\text { (etch rate } 0.5 \mathrm{~nm} / \mathrm{s}, 6 \mathrm{~min} \text { ) }\end{array}$ & \\
\hline 16 & & - Quick dump rinse & \\
\hline 17 & & - Single wafer spin dryer & \\
\hline 18 & \multirow{2}{*}{$\begin{array}{l}\text { Sputtering Ta-Pt } \\
\text { Sputterke } \\
\text { - Base pressure: } \\
\text { < 1.0e-6 mbar } \\
\text { - Sputter pressure: } \\
6.6 \mathrm{e}-3 \mathrm{mbar} \\
\text { - Power: } 200 \mathrm{~W} \\
\text { - Ar flow to adjust } \\
\text { pressure }\end{array}$} & $\begin{array}{l}\text { - Sputter Ta } \\
\text { (deposition rate } 16.5 \mathrm{~nm} / \mathrm{min} \text { ) } \\
\text { Deposition time for } 30 \mathrm{~nm}=109 \mathrm{~s} \\
\text { after } 1 \text { min pre-sputter. }\end{array}$ & \\
\hline 19 & & $\begin{array}{l}\text { - Sputter Pt } \\
\text { (deposition rate } 22-27 \mathrm{~nm} / \mathrm{min} \text { ) } \\
\text { Deposition time for } 120 \mathrm{~nm}=288 \mathrm{~s} \\
\text { after } 1 \text { min pre-sputter. }\end{array}$ & \\
\hline 20 & \multirow[t]{4}{*}{$\begin{array}{l}\text { Lift-off } \\
\text { Wetbench } 11\end{array}$} & $\begin{array}{l}\text { - Lift-off in acetone } \\
\text { (technical, 1-2 h) }\end{array}$ & \\
\hline 21 & & $\begin{array}{l}\text { - Spray with } \mathrm{VLSI} \text { acetone to remove } \\
\text { remaining resist and } \mathrm{Ta} / \mathrm{Pt}\end{array}$ & \\
\hline 22 & & - Spray with VLSI isopropanol & \\
\hline 23 & & - Single wafer spin drying & \\
\hline & \multirow{7}{*}{$\begin{array}{l}\text { Powderblast foil } \\
\text { lamination, } \\
\text { exposure and } \\
\text { development } \\
\text { Laminator, EVG620, } \\
\text { Dicing room and } \\
\text { BIOS CR2516 }\end{array}$} & BF410 Foil lamination & \\
\hline 24 & & $\begin{array}{l}\text { - Roll BF410 foil (remove protection } \\
\text { foil and roll on the backside of the } \\
\text { BF33 wafer) }\end{array}$ & \\
\hline 25 & & $\begin{array}{l}\text { - Laminate between } 2 \text { sheets of } \\
\text { paper }\left(105^{\circ} \mathrm{C} \text {, roller speed } 2\right) \text {. After } \\
\text { lamination, cut to size. }\end{array}$ & \\
\hline & & Foil exposure & \\
\hline \multirow{3}{*}{26} & & $\begin{array}{l}\text { - Exposure: Hg-lamp } \\
\text { (12 mW/cm², 20s) }\end{array}$ & \\
\hline & & $\begin{array}{l}\text { - Bottom-side, crosshairs, } \\
\text { soft-contact, foil } 100 \mu \mathrm{m} \\
\text { - Mask 4, alignment marker [2-3], } \\
\text { wafer face-down }\end{array}$ & \\
\hline & & Protection foil lamination & \\
\hline
\end{tabular}




\begin{tabular}{|c|c|c|}
\hline \multirow[t]{2}{*}{27} & & $\begin{array}{l}\text { - Laminate dicing foil (on the process } \\
\text { side of the BF33 wafer, remove } \\
\text { BF410 protection layer after bake at } \\
70^{\circ} \mathrm{C} \text { for } 30 \text { s) }\end{array}$ \\
\hline & & Development \\
\hline 28 & & $\begin{array}{l}\text { - Rinse with } 2 \% \mathrm{Na}_{2} \mathrm{CO}_{3} \text { solution (40 } \\
{ }^{\circ} \mathrm{C} \text { ) until developed parts lift-off. }\end{array}$ \\
\hline 29 & & $\begin{array}{l}\text { - Spin dry (60s) and dry on hot plate } \\
\left(70^{\circ} \mathrm{C}, 60 \mathrm{~s}\right)\end{array}$ \\
\hline 30 & $\begin{array}{l}\text { Powderblasting } \\
\text { CR } 5^{\text {th }} \text { floor }\end{array}$ & $\begin{array}{l}\text { - Grain size: } 29 \mu \mathrm{m} \\
\text { - Step size: } 0.5 \mu \mathrm{m} \text {. } \\
\text { Speed: } 10 \mathrm{~mm} / \mathrm{s} \text {. } \\
\text { - } 500 \mu \mathrm{m} \text { wafer: } 1 \text { run at } 5 \mathrm{bar} \\
\text { - } 1100 \mu \mathrm{m} \text { wafer: } 1 \text { run at } 5 \mathrm{bar} \\
+1 \text { run at } 3 \text { bar or } 2 \text { runs at } 5 \text { bar } \\
\text { with } 2 \text { layers of BF } 410 \text { foil }\end{array}$ \\
\hline 31 & \multirow[t]{2}{*}{$\begin{array}{l}\text { Delamination and } \\
\text { cleaning } \\
\text { BIOS CR2615 }\end{array}$} & $\begin{array}{l}\text { - Remove BF410 and dicing foil by } \\
\text { immersing in warm water }\left( \pm 40^{\circ} \mathrm{C}\right) \text {. } \\
\text { Peel foils in parallel with respect to } \\
\text { the wafer's surface to prevent } \\
\text { breaking. }\end{array}$ \\
\hline 32 & & $\begin{array}{l}\text { - Remove particles by ultrasound in } \\
\text { DI ( } 30 \mathrm{~min})\end{array}$ \\
\hline 33 & \multirow{3}{*}{$\begin{array}{l}\text { Removal of } \\
\text { particles } \\
\text { Wetbench } 11\end{array}$} & $\begin{array}{l}\text { - Dipping in 99\% } \mathrm{HNO}_{3} \\
\text { (beaker 1, 20s-30s) }\end{array}$ \\
\hline 34 & & - Quick dump rinse \\
\hline 35 & & - Single wafer spin drying \\
\hline 36 & \multirow{7}{*}{$\begin{array}{l}\text { PerMX3020 foil } \\
\text { lamination, } \\
\text { exposure and } \\
\text { development } \\
\text { Laminator, EVG620, } \\
\text { Wetbench } 24\end{array}$} & - UV-Ozone cleaning (5 min) \\
\hline 37 & & - Bake $\left(120^{\circ} \mathrm{C}, 10 \mathrm{~min}\right)$ \\
\hline 38 & & PerMX3020 lamination \\
\hline 39 & & $\begin{array}{l}\text { - Roll PerMX3020 foil (remove } \\
\text { protection foil very gently and roll on } \\
\text { the process side of the wafer) }\end{array}$ \\
\hline 40 & & $\begin{array}{l}\text { - Laminate between } 2 \text { sheets of } \\
\text { paper }\left(80^{\circ} \mathrm{C} \text {, roller speed } 2\right) \text {. After } \\
\text { lamination, cut to size. Don't remove } \\
\text { the protection layer. }\end{array}$ \\
\hline \multirow[t]{2}{*}{41} & & $\begin{array}{l}\text { - Pre-bake } \\
\left(50^{\circ} \mathrm{C}-5 \mathrm{~min}, 65^{\circ} \mathrm{C}-5 \mathrm{~min}, 95^{\circ} \mathrm{C}-10 \mathrm{~min}\right)\end{array}$ \\
\hline & & Foil exposure \\
\hline
\end{tabular}




\begin{tabular}{|c|c|c|}
\hline 42 & $\begin{array}{l}\text { - Interval exposure: Hg-lamp (12 } \\
\mathrm{mW} / \mathrm{cm}^{2}, 8 \times 5 \mathrm{~s} \text { ) } \\
\text { - Top-side, crosshairs, soft-contact, } \\
\text { foil } 20 \mu \mathrm{m} \\
\text { - Mask } 1 \text {, alignment marker [1-1], } \\
\text { wafer face-up }\end{array}$ & \\
\hline 43 & - Remove protection foil. & \\
\hline \multirow[t]{2}{*}{44} & $\begin{array}{l}\text { - Post-exposure bake }\left(50^{\circ} \mathrm{C}-10 \mathrm{~min} \text {, }\right. \\
\left.65^{\circ} \mathrm{C}-10 \mathrm{~min}, 95^{\circ} \mathrm{C}-20 \mathrm{~min}\right) . \\
\text { Wait until }<50^{\circ} \mathrm{C}\end{array}$ & \\
\hline & Development & \\
\hline 45 & $\begin{array}{l}\text { - Spray develop with RER-600 (3 } \\
\text { runs, 20s). Don't spray RER-600 } \\
\text { directly on the wafer. }\end{array}$ & \\
\hline 46 & $\begin{array}{l}\text { - Gently spray VLSI-IPA to remove } \\
\text { RER-600. }\end{array}$ & \\
\hline 47 & - Single wafer spin drying & \\
\hline
\end{tabular}

\section{Alignment and bonding}

\begin{tabular}{|c|c|c|c|}
\hline & \multicolumn{2}{|c|}{ Process description } & Cross-section \\
\hline 1 & $\begin{array}{l}\text { Stack alignment } \\
\text { EVG } 620 \text { \& anodic } \\
\text { bond chuck }\end{array}$ & $\begin{array}{l}\text { - Load wafers in the anodic bond } \\
\text { chuck. Load } 1100 \mu \mathrm{m} \text { wafer first, } \\
\text { process side face-down. Load } 500 \\
\mu \mathrm{m} \text { wafer next, process side face-up. } \\
\text { - Anodic bond program, crosshairs, } \\
\text { flag before WEC, align before WEC } \\
\text { - Align using alignment marker [2,2]. } \\
\text { - Don't remove flags }\end{array}$ & \\
\hline 2 & $\begin{array}{l}\text { Pre-bonding } \\
\text { Anodic bonder }\end{array}$ & $\begin{array}{l}\text { - Adjust stack thickness to } 4.72 \mathrm{~mm} \\
\text { - Select bond program “Bjorn-SU8-1" } \\
\text { - Wait until pressure < } 10^{-1} \mathrm{mbar} \text {. } \\
\text { - Increase temperature to } 150{ }^{\circ} \mathrm{C} \\
\text { - Move piston (pressure } 1000 \mathrm{~N} \text { ) } \\
\text { - Waiting step for pre-bond (10s) } \\
\text { - Remove flags } \\
\text { - Bond for } 30 \mathrm{~min}\end{array}$ & \\
\hline 3 & $\begin{array}{l}\text { Bonding } \\
\text { Carver heat press } \\
\text { (BIOS) }\end{array}$ & $\begin{array}{l}\text { - Load stack and increase } \\
\text { temperature to } 150{ }^{\circ} \mathrm{C} \\
\text { - Apply } 1 \text { ton of pressure }(5 \mathrm{~min})\end{array}$ & \\
\hline
\end{tabular}




\begin{tabular}{|c|c|c|}
\hline & & $\begin{array}{l}\text { - If air is remaining within the stack, } \\
\text { increase pressure to } 1.5 \text { or } 2 \text { tons. } \\
\text { - Bond for } 1 \mathrm{~h}\end{array}$ \\
\hline 4 & $\begin{array}{l}\text { Dicing } \\
\text { Disco-DAD dicing } \\
\text { saw }\end{array}$ & $\begin{array}{l}\text { - Mount wafer stack in holder using } \\
\text { transparent dicing foil }(125 \mu \mathrm{m}) \\
\text { - TC-300 blade, RPM 25k, } \\
\text { speed } 3 \mathrm{~mm} / \mathrm{s} \\
\text { - Stack } 1620 \mu \mathrm{m} \text { thick } \\
\text { Wafer size: } 110 \mathrm{~mm} \\
\text { - x-translation }=20.3 \mathrm{~mm} \text {, } \\
\text { y-translation = } 18.3 \mathrm{~mm} \\
\text { - Cut stack in } 1 \text { run }\end{array}$ \\
\hline
\end{tabular}




\section{Appendix B - ECM in Matlab}

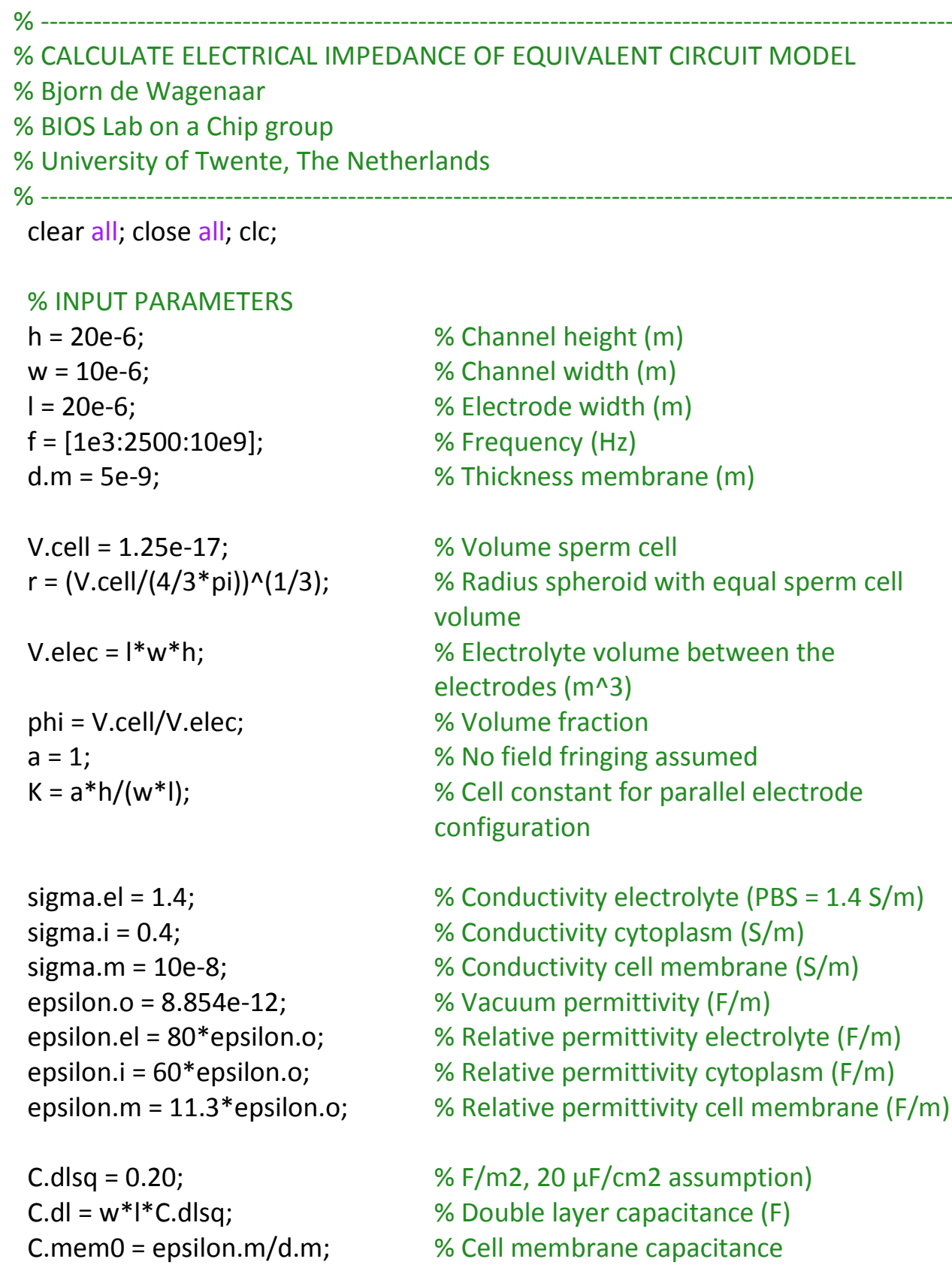

$\%$ Channel height (m)

$\%$ Channel width (m)

$\%$ Electrode width (m)

$\%$ Frequency $(\mathrm{Hz})$

$\%$ Thickness membrane (m)

$\%$ Volume sperm cell

$\%$ Radius spheroid with equal sperm cell volume

$\%$ Electrolyte volume between the

electrodes $\left(\mathrm{m}^{\wedge} 3\right)$

$\%$ Volume fraction

$\%$ No field fringing assumed

$\%$ Cell constant for parallel electrode configuration

$\%$ Conductivity electrolyte (PBS = $1.4 \mathrm{~S} / \mathrm{m}$ )

$\%$ Conductivity cytoplasm (S/m)

$\%$ Conductivity cell membrane $(\mathrm{S} / \mathrm{m})$

$\%$ Vacuum permittivity (F/m)

$\%$ Relative permittivity electrolyte (F/m)

$\%$ Relative permittivity cytoplasm (F/m)

$\%$ Relative permittivity cell membrane (F/m)

$\% \mathrm{~F} / \mathrm{m} 2,20 \mu \mathrm{F} / \mathrm{cm} 2$ assumption)

$\%$ Double layer capacitance (F)

$\%$ Cell membrane capacitance 


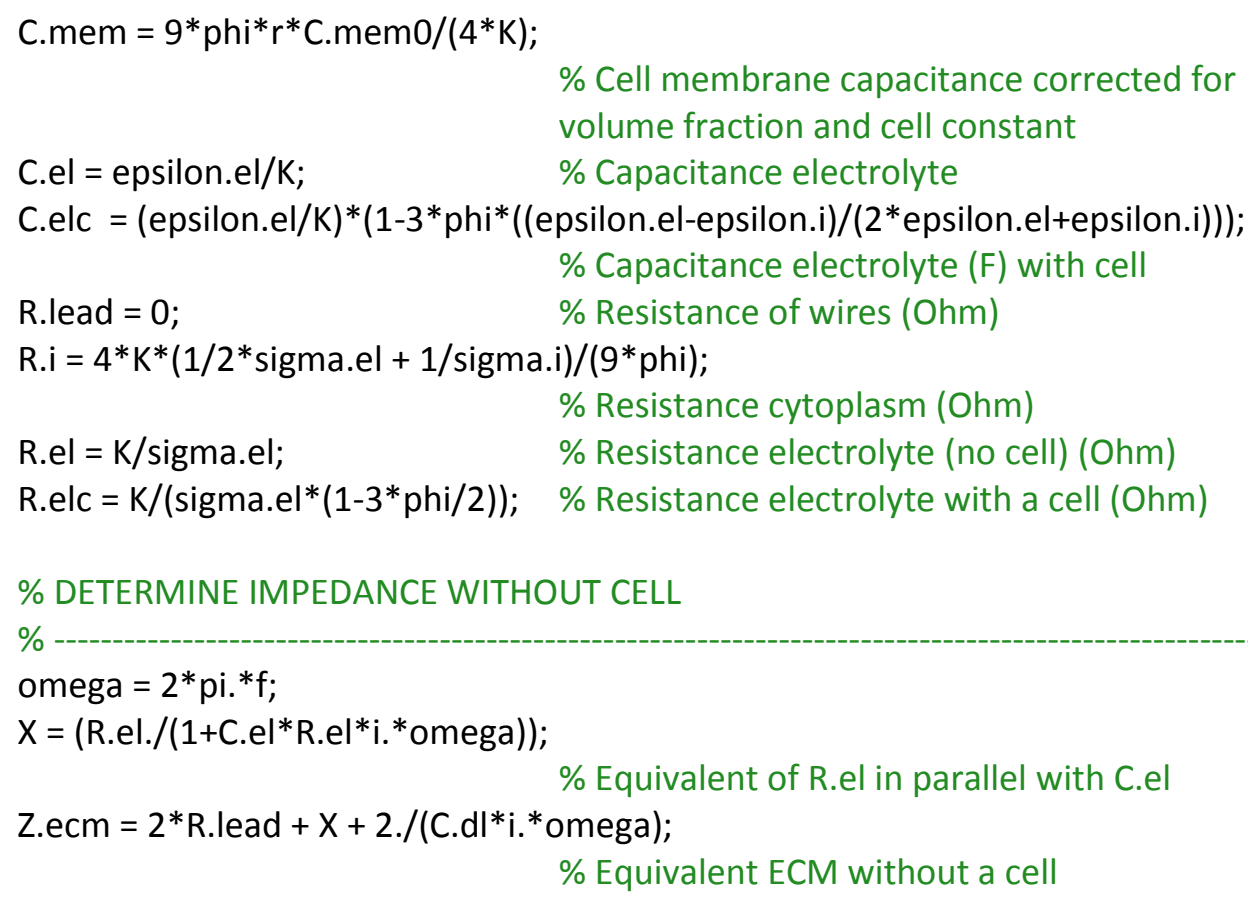


174 Appendix B

figure(2);

semilogx(f,phase.ecm, 'b--','LineWidth',2)

hold on;

semilogx(f,phase.ecm_cell,'g--','LineWidth',2)

legend('PBS','PBS with cell')

xlabel('Frequency $(\mathrm{Hz})$ ');

ylabel('Phase $\left({ }^{\circ}\right)$ '); 


\section{Samenvatting}

Microfluïdische technologie wordt steeds frequenter toegepast voor de analyse en scheiding van spermacellen. Verschillende onderzoeken hebben laten zien dat deze technologie zeer geschikt is voor het sorteren van spermacellen op basis van hun motiliteit. Met de beschreven en gebruikte methoden worden vele spermacellen gelijktijdig gesorteerd, die onderling nog veel van elkaar kunnen verschillen. Naast deze bulk methoden, kan de analyse van individuele spermacellen erg interessant zijn voor sperma selectie bij geassisteerde voortplantingstechnieken en voor de opwerking van sperma ten bate van de kunstmatige inseminatie in de veeteelt. Een interessante manier om individuele spermacellen te onderzoeken in een microfluïdisch systeem, is het uitvoeren van non-invasieve elektrische analyse met behulp van geïntegreerde meetelektroden.

Om een cel op individueel niveau uitvoerig te onderzoeken, moet deze op een nauwkeurige plaats gepositioneerd blijven gedurende analyse. Veel middelen zijn beschikbaar om individuele cellen (tijdelijk) te positioneren, gebruik makend van hydrodynamische, elektrische, chemische, optische, akoestische en magnetische methoden (hoofdstuk 2). Elk van deze methoden heeft zijn eigen voor- en nadelen. Aangezien het elektrisch karakteriseren van individuele gevangen spermacellen een van de doelen was van dit project, is er een eisen lijst opgesteld om de meest geschikte methode te bepalen. De gekozen methode moet in staat zijn om de sperma cel te vangen op een stabiele, reversibele en niet invasieve manier. Daarnaast moet de methode geïntegreerd kunnen worden met micro-elektroden voor het uitvoeren van elektrische analyses. Op basis van deze opgestelde eisen is een hydrodynamische methode verkozen als meest geschikt voor het vangen van individuele spermacellen.

Elektrische analyse van cellen in microfluïdische systemen is in het algemeen gebaseerd op het meten van veranderingen in de impedantie wanneer een cel tussen twee microelektroden geplaatst wordt (hoofdstuk 3). Deze verandering kan informatie bevatten over de celgrootte, het membraan en/of het cytoplasma. Echter moet er ook rekening gehouden worden met de invloed van de geometrie van het microfluïdische systeem op de gemeten veranderingen in de elektrische impedantie. Bij lage frequenties wordt de impedantie voornamelijk bepaald door de capaciteit van de elektrische dubbellaag, terwijl bij hoge frequenties de parasitaire effecten een grote rol spelen. De beste meetfrequentie wordt bepaald 
door de geometrie van het systeem in combinatie met de geleidbaarheid van het medium en bevindt zich op een frequentie, waarbij de totale impedantie van het systeem voornamelijk bepaald wordt door de weerstand van het elektrolyt. De invloed van de geometrie op de elektrische impedantie kan eenvoudig bepaald worden door het opstellen van een equivalent netwerk.

Twee meettechnieken, die regelmatig gebruikt worden voor de elektrische analyse van individuele cellen in microfluïdische systemen, zijn microfluïdische impedantie cytometrie en spectroscopie. In het geval van microfluïdische impedantie cytometrie, stromen cellen door een microfluïdisch kanaal waarin ten minste één electrode paar aanwezig is voor het meten van veranderingen in impedantie bij ten minste één meetfrequentie. Impedantie cytometrie is toegepast voor het onderscheiden van verschillende cel types in eenzelfde celpopulatie en voor de analyse van de cel vitaliteit, morfologie en differentiatie. In het geval van microfluïdische impedantie spectroscopie wordt typisch een enkele cel gevangen, terwijl de elektrische impedantie wordt gemeten over een breed frequentiebereik.

Het ontwerp en vervaardiging van een microfluïdisch systeem voor het hydrodynamisch vangen van individuele spermacellen is beschreven in hoofdstuk 4. In dit systeem zijn individuele spermacellen gevangen in kleine microfluïdische zijkanalen, die twee grotere hoofdkanalen verbinden. Door het aanbrengen van een drukverschil over deze zijkanalen ontstaat een vloeistofstroom door deze kanalen, die in staat is om individuele spermacellen te vangen bij de kanaalingang. $\mathrm{Na}$ experimenten met verschillende zijkanaal hoogtes, bleek een hoogte van $1 \mu \mathrm{m}$ meest geschikt te zijn voor het vangen van individuele spermacellen. $\mathrm{Na}$ het invangen zijn de plasma membranen, acrosoom membranen en het type geslachtschromosoom in de individuele cellen onderzocht met behulp van fluorescente aankleuringen.

Om de motiliteit van individuele spermacellen te onderzoeken m.b.v. elektrische analyse, is het vangsysteem geïntegreerd met micro-elektroden (hoofdstuk 5). Tijdens het invangen van individuele spermacellen is de impedantie continu en differentieel opgenomen bij een meetfrequentie van $1 \mathrm{MHz}$. Wanneer een cel tussen de meetelektroden periodiek bewoog, werd een oscillatie waargenomen in het elektrische signaal. $\mathrm{Na}$ het registreren van deze data is een Fast Fourier Transformatie uitgevoerd om de frequentiecomponenten in dit signaal te onderzoeken. Deze analyse liet een stabiele frequentie zien in het signaal die sterk gerelateerd was aan de slag frequentie van de staart van de cel, welke bepaald is met een optische methode. De robuustheid van de elektrische detectie methode is 
getest door de slag frequentie van de cellen te variëren door temperatuursveranderingen en blootstelling aan cafeïne. In beide situaties kon het effect op de slag frequentie elektrische bepaald worden.

Naast het uitvoeren van elektrische analyse bij statische condities, kunnen spermacellen ook onderzocht worden onder dynamische condities. Hiervoor is een microfluïdisch platform ontworpen en gefabriceerd om de cellen met behulp van impedantie cytometrie te onderzoeken. Hierbij stromen de spermacellen tussen een paar micro-elektroden, terwijl veranderingen in impedantie worden gemeten (hoofdstuk 6). Deze aanpak is gebruikt om een specifieke morfologische afwijking van spermacellen in kaart te brengen, ofwel de aanwezigheid van een cytoplasmatische druppel op de staart van spermacellen. Allereerst is de impedantie differentieel gemeten bij een meetfrequentie van 1.3 $\mathrm{MHz}$ terwijl de spermacellen door een klein microfluïdisch kanaal (20 bij $20 \mu \mathrm{m}$ ) stroomden. De vorm van de impedantie verandering over de tijd verschafte informatie over de oriëntatie van de sperma cel (kop of staart eerst). Wanneer een cytoplasmatische druppel aanwezig was op de staart van de cel, was een extra schouder in het impedantiesignaal aanwezig, wat een vergroting van de oppervlakte onder de curve tot gevolg had. Na normalisatie van deze oppervlakte, is een significant verschil gevonden tussen een populatie cellen met en zonder cytoplasmatische druppel. Met behulp van een ROC curve is het onderscheidingsvermogen van de elektrische detectie methode onderzocht, waarbij een oppervlakte onder de curve van 0.85 is gevonden.

Met behulp van elektrische detectie zijn verschillende parameters van spermacellen te onderzoeken. Het sorteren tussen spermacellen met goede eigenschappen en spermacellen met mindere eigenschappen kan grote voordelen opleveren ten opzichte van de huidige sperma opwerkingstechnieken. Om dit te realiseren, is een sorteersysteem ontworpen, dat in staat is om de spermacellen te sorteren door middel van diëlektroforese op basis van de opgenomen veranderingen in impedantie (hoofdstuk 6). Dit sorteersysteem bestaat uit drie verschillende elementen: focussering, impedantie detectie en sortering van spermacellen. Ten eerste zijn de spermacellen gefocusseerd met dielektroforese door 4 electroden. Door het exciteren van de focusseer electroden met een $10 \mathrm{MHz}$ $3 \mathrm{~V}$ sinus-signaal werden de langsstromende cellen naar het midden van het kanaal geduwd. Dit resulteerde in een hogere uniformiteit van de cel-locatie en -snelheid. $\mathrm{Na}$ het focusseren, is de impedantie van langsstromende cellen gemeten. In een eerste experiment stroomde een mix van $3 \mu \mathrm{m}$ plastic bolletjes en spermacellen 
door de chip terwijl de impedantie werd gemeten. Een duidelijk onderscheid kon gemaakt worden tussen plastic bolletjes en spermacellen gebaseerd op de absolute verandering in impedantie, waardoor de bolletjes effectief en selectief gescheiden konden worden. Dit sorteersysteem kan mogelijk gebruikt worden om spermacellen met een ongewenst morfologie uit het semen te verwijderen.

Het manipuleren van spermacellen met dielektroforese is tot nu toe nog niet uitvoerig onderzocht. Om er zeker van te zijn dat de spermacellen bestand zijn tegen de opgelegde elektrische velden, is de sperma kwaliteit onderzocht na blootstelling aan elektrische velden in een microfluïdische chip (hoofdstuk 7). De kwaliteit van de cellen is onderzocht door de fluorescente aankleuring van de plasma membranen, de acrosoom membranen en de mitochondriale membranen. Hoogfrequente elektrische velden ( $10 \mathrm{MHz}$ ) hadden geen schadelijke invloed op de spermacellen, alhoewel minieme schade is geobserveerd door het gebruik van een microfluïdische chip. Omvangrijke schade is geobserveerd wanneer de frequentie werd verlaagd tot waarden dicht bij gelijkspanning $(10 \mathrm{~Hz})$. Deze bevindingen komen overeen met simulaties die uitgevoerd zijn, waarbij het transmembraan potentiaal berekend is. Deze resultaten duiden op dat dielektroforese veilig is toe te passen voor het sorteren van spermacellen in een microfluïdisch systeem. 


\section{List of publications}

\section{Journal articles (this thesis)}

de Wagenaar, B., van Etten, J., Olthuis, W., van den Berg, A., Segerink, L.I., Sperm quality after DEP manipulation. Manuscript in preparation.

de Wagenaar, B., Geijs, D.J., de Boer, H.L., Bomer, J.G., Olthuis, W., van den Berg, A., Segerink, L.I., Spermometer: electrical characterization of single sperm motility. Submitted to Fertility and Sterility.

de Wagenaar, B., Dekker, S., de Boer, H.L., Bomer, J.G., Olthuis, W., van den Berg, A., Segerink, L.I., Towards microfluidic sperm refinement: impedance-based analysis and sorting of sperm cells. Submitted to Lab on a Chip.

de Wagenaar, B., Berendsen, J.T.W., Bomer, J.G., Olthuis, W., van den Berg, A., Segerink, L.I., Microfluidic single sperm entrapment and analysis. Lab Chip, 2015. 15(5): p. 1294-301.

\section{Other articles}

Frimat, J.P., Bronkhorst, M., de Wagenaar, B., Bomer, J.G., van der Heijden, F., van den Berg, A., Segerink, L.I., Make it spin: individual trapping of sperm for analysis and recovery using micro-contact printing. Lab Chip, 2014. 14(15): p. 2635-41.

Sukas, S., Schreuder, E., de Wagenaar, B., Swennenhuis, J.F., van den Berg, A., Terstappen, L.W.M.M., Le Gac, S., A novel side electrode configuration integrated in fused silica microsystems for synchronous optical and electrical spectroscopy, Lab Chip, 2014. 14(11): p. 1821-25.

Kandhai-Ragunath, J.J., Jørstad, H.T. de Wagenaar, B., de Man, F.H., Stoel, M.G., van Es, J., Doelman, C.J., Doggen, C.J., Peters, R.J., von Birgelen, C., Assesment of the relation between initial culprit vessel patency in acute ST-elevation myocardial infarction and endothelial function, Eurolntervention, 2014, 10(7): p. 784-91.

Griep, L.M., Wolbers, F., de Wagenaar, B., ter Braak, P.M., Weksler, B.B., Romero, I.A., Couraud, P.O., Vermes, I., van der Meer, A.D., van den Berg, A., BBB on chip: microfluidic platform to mechanically and biochemically modulate blood-brain barrier function, Biomed Microdevices, 2013. 15(1): p. 145-50. 


\section{Patents}

de Wagenaar, B., Segerink, L.I., Olthuis, W., Sprenkels, A.J. and van den Berg, A. Microfluidic device for selection of semen. P100207EP00, submitted 30-09-2015.

\section{Conference contributions}

de Wagenaar, B., Geijs, D.J., de Boer, H.L., Bomer, J.G., Olthuis, W., van den Berg, A., Segerink, L.I. Electrical analysis of single sperm motility. MicroNanoConference (oral presentation), December 8-9, 2015, Amsterdam, The Netherlands.

de Wagenaar, B., Dekker, S., van Etten, J., Bomer, J.G., van Nieuwkasteele, J.W., Olthuis, W., van den Berg, A., Segerink, L.I., Towards microfluidic sperm refinement: continuous flow label-free analysis and sorting of sperm cells. MicroTAS (poster presentation), October 25-29, 2015, Gyeongju, South-Korea.

de Wagenaar, B. Berendsen, J.T.W., Huisintveld, N., Olthuis, W. van den Berg, A. Segerink, L.I., Microfluidic single sperm entrapment and analysis. EMBS Micro and Nanotechnology in Medicine Conference (poster presentation), December 8-12, 2014, Oahu, HI, USA.

de Wagenaar, B., Berendsen, J.T.W., Olthuis, W., van den Berg, A., Segerink, L.I., Microfluidic single sperm entrapment and analysis. NanoCity (oral presentation), October 27-28, 2014, Utrecht, The Netherlands.

de Wagenaar, B., Segerink, L.I., Sprenkels, A.J., van den Berg, A. Label-free cell cycle analysis by impedance sensing. NanoBioTech (poster presentation), November 12-14, 2012, Montreux, Switzerland. 


\section{Acknowledgements}

Welcome to the final part of my thesis where my last words might be your first. During my PhD project, I got help from a lot of people, which I want to thank accordingly.

First of all, I want to thank Albert for the position within the group and for funding my project. The whole story started when Albert allowed me to join the BIOS workweek to the United States. In the end, this trip turned out to be quite a page turner and defined the path I'm walking today. During this workweek, I got inspired by tiny chips, which could in theory replace complete labs. This interest made me to decide to look for a graduation project within the BIOS group. After a short informal talk in a sports bar in Boston, Albert advised me to talk to Andries, who offered me an assignment in a starting project within the group. During my graduation, I experienced a nice working atmosphere in which people were very collegially and knew how to make a little fun once in a while. This atmosphere definitely triggered my enthusiasm to start a PhD project in the BIOS group.

I want to thank Loes for all her work as a daily supervisor of my project. Already during my master assignment, you helped me a lot in getting familiar with the field of impedance spectroscopy. During my PhD, you always knew how to motivate me, even when we communicated via a Skype call from Stockholm. Furthermore, I'm grateful to you for teaching me, in my opinion, the most important lesson during a PhD-project.

I also want to show my gratitude to Wouter. Halfway my project, you joined as a daily supervisor. You were able to catch up with months and months of research very quickly, even when I was occasionally rushing things during meetings. In short, you were always punctual and well prepared for each meeting, in which I enjoyed your keen eye on scientific research. You introduced a lot of structure in my way of working, which contributed heavily to the completion of this thesis. Furthermore, your door was always open for a short question or for some advice.

I want to thank Ad for being my daily supervisor for the first part of my project. You are a real guru in the field of electrical engineering and your tips and tricks have definitely payed off. I will never forget the moment I showed you my first soldered circuit, which in my opinion was kind of neat, while you responded with something like, "It's a bit sloppy, but it will probably do the trick". Your positive attitude and motivation always helped me to do my best. 
During my research, I got a lot of technical support which has been essential. I want to thank Johan for all the work in the cleanroom and all his knowhow about chip design and fabrication. Furthermore, I want to thank Hans for the fabrication of my Faraday cage, alignment setup, chip holder and much more. Furthermore, a year ago I broke the microscope and you fixed it in no time, which saved me a lot of valuable time. I also want to thank Jan van N. for fabricating my custom chip holder, which worked perfectly from the start. Furthermore, I want to thank Fleur for her assistance in chip fabrication. Finally, I want to thank Paul for all his help in the bio lab.

Also, I want to thank Jan E. for his help in reviewing and correcting my abstract submissions. With your help, my abstracts got accepted for EMBS MNM'14 (Hawaii) and MicroTAS'15 (South-Korea) conferences, which both were amazing experiences. Furthermore, I want to thank Jean-Phillipe and Sertan for a nice collaboration, scientific discussion and motivational speeches. I also want to thank Evelien for picking up semen samples at the KI Twenthe during the first years and Jan Nijland for his assistance in sample delivery.

During my PhD, I had several students who helped me with my research. I'm very grateful for all of their good work. Stefan, Jorien, Jimmy, Daan, Nik, Marleen and Inge, thanks a lot!

Finally, I want to thank Rogier, Sertan, Alexander, Trieu and Yawar for being great office mates. And not to forget, I want to thank my roommates Marco and Sieger for listening to all my complaints during periods of struggle.

Besides the hard work, the last 4.5 years have also been a lot of fun. Especially, playing music with Lennart, Rogier and Flories has been awesome. I loved our weekly jam and our "gigs" during the BBQ's in Albert's garden after the annual MTB event. And I will never forget the moment we were served cognac after playing our song "Where's my whisky". So guys, thanks!

Furthermore, I had a lot of fun during various BIOS events. Playing football was definitely one of them and therefore I want to thank Rogier, Lennart, Paul, Martijn, Laura, Yawar, Marciano, Hugo, Egbert, Jean-Phillipe, Miquel and all the others which I'm forgetting.

I had the luxury to join the BIOS workweeks to the US, France and the UK. These weeks have both been fun and inspirational and I want to thank everyone who helped organizing these events. 
When talking about fun, I cannot forget the BIOS sailing weekends. The sailing, the BBQ's, the (bon)Fires, "De grootste buisgolf Ever", etc. Lennart, Paul and Jan van N., thanks for the organization!

Finally, I want to thank all the people in the BIOS group for all their support and for creating a nice working atmosphere!

And last but not least, I want to thank Carla and my family for their support during the last 4.5 years. Your support has made all the difference!

Bjorn de Wagenaar University of Twente February 2016 
